Thesis for the degree of Doctor of Philosophy

\title{
Dispersion tailoring in both integrated photonics and fiber- optic based devices
}

Sara Mas Gómez

Supervisors:

Javier Martí Sendra

Jesús Palací López

Valencia, June 2015 



\section{Agradecimientos}

Esta Tesis se la quiero dedicar en especial a mi padre porque 'es muy sencillo de entender'. A mi madre, a mis hermanos y a mi cuñada, por todo su apoyo y cariño incondicional. A mis sobrinos, Dani y Jose, porque son lo más bonito del mundo.

A mi director de Tesis Dr. Javier Martí por todas las directrices, consejos e ideas que me ha aportado durante estos años. A mi co-director Dr. Jesús Palací por su apoyo y ánimo constante y por todos los momentos en los que hemos 'revolucionado la ciencia' a base de Aquarius, cervezas y anchoas. A toda la gente del NTC, en especial a mi 'bro' Luis por su alegría incansable y contagiosa, a Sergio por sus estadísticas imposibles y juegos de palabras, a Marghe por su paciencia infinita, a Álvaro por ser el mejor compañero de futbolín de Alginet y más allá, a Alba por los ratos pasados en el laboratorio y a toda la pandilla de la hora de la comida Mario, Diego, Álex, Fede, Pau, Ángela, Andrés y Javi. A Julio y a Antoine por toda la ayuda y dudas resueltas siempre con una sonrisa. Mil millones de gracias a David Zurita, por todas las idas y venidas, reparaciones y consultas en los laboratorios. Gracias también al gran Amadeu Griol, por las infinitas muestras, intentos y modificaciones. A los grandes que ya se fueron del NTC, Fede 'el argentino', Claudio, Joaquín, Guillermo, Jordi, Mercé, Begoña, Javi, Pak, Josema y Pere.

A mi gente de siempre Ana, Stephanie, Ainhoa, Alba, Aida, David, Óscar, Jéssica, Mari y Layu, a las cinque de 'Cinque Terre' Sandra, Esther, Nuria, Carlita y Celia y a los nuevos fichajes Esther, Tom, Lola, Viki, Churro y Rita. Con mucho cariño a Salvador por su infinita hospitalidad, a Amparo por su incomparable arroz con conejo, a Enriqueta, Daniel, Merche, Servando y Águeda, así como a Daniela por todos los 'planes' que hacemos. A Miniyo por estar ahí siempre siempre siempre y porque 'you're simply the best'.

A los que ya no están pero no se olvidan: A mis abuelos, os llevo presentes siempre. Al maestro Dr. José Caraquitena, porque fuiste el origen de todo esto.

A todos, gracias. 



\section{Table of contents}

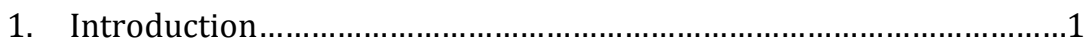

1.1. Optical Communication Systems ..............................................

1.2. Chromatic Dispersion Fundamentals ......................................12

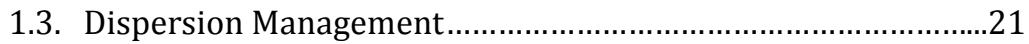

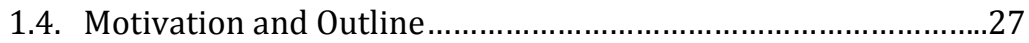

2. Integrated Photonics.......................................................................

2.1. Dispersion Tailoring in Integrated Waveguides........................33

2.2. Experimental Dispersion Measurements ....................................34

3. Tapered Fibers...............................................................................

3.1. Dispersion Tailoring in Tapered Fibers ....................................43

3.2. Experimental Dispersion Measurements.................................44

3.3. Tapered Fiber Applications....................................................4

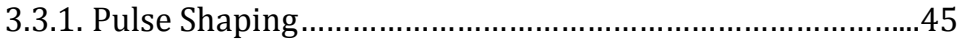

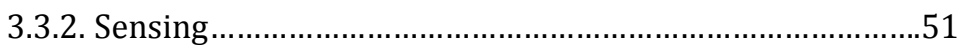

3.3.3. Mode Conversion..........................................................

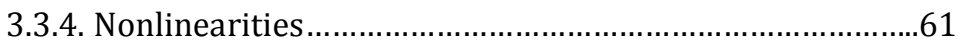

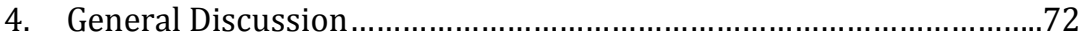

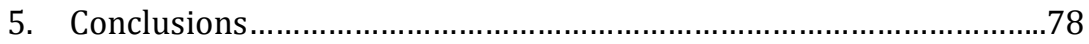

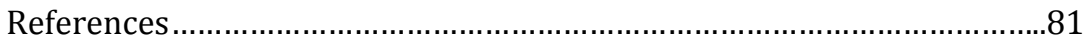

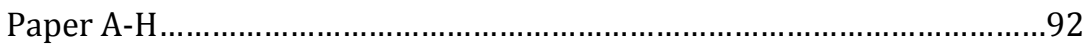

Paper A

Paper B

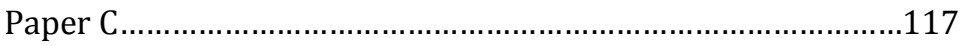

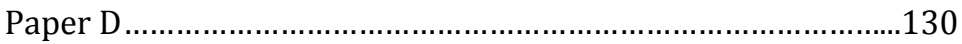

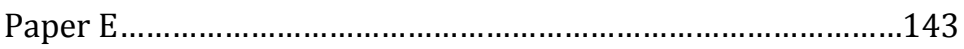

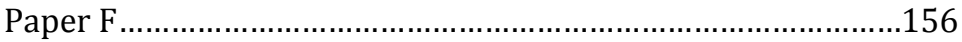

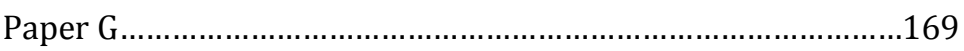

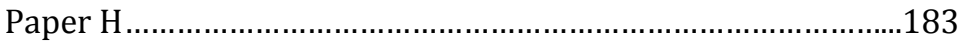




\section{Abstract}

This Thesis focuses on the study, implementation and characterization of chromatic dispersion tailoring employing both optical fiber and photonic integrated waveguides. Chromatic dispersion causes that the different spectral components of an optical pulse travel at different velocities. This effect can be separated into two different fundamental contributions, material dispersion and waveguide dispersion. Chromatic dispersion can be tailored through the design of the structural parameters of the device in order to obtain specific characteristics in the resulting dispersion profile such as low values of dispersion and/or zero dispersion at a desired wavelength, for example. This approach is very useful in dispersion-dependent applications. In this $\mathrm{PhD}$, we investigate chromatic dispersion tailoring in two different transmission mediums, photonic integrated waveguides and optical fiber.

In the first case, two different geometries of Silicon-on-Insulator (SOI) integrated waveguides, strip and slot, are considered. By varying structural parameters such as the cross-section, aspect ratio or fill factor, different chromatic dispersion profiles are obtained. In addition, the influence of the slot location is evaluated. This study is carried out using simulation software in order to obtain the effective refractive index profile as a function of wavelength, which is later differentiated to obtain the final dispersion values. Besides, chromatic dispersion in both waveguide geometries is experimentally measured using an interferometer technique.

In the second case, the chromatic dispersion present in a tapered fiber is studied. A tapered fiber consists of a narrow waist located between two transition regions and it allows the modification of the conventional propagation conditions due to the interference between the modes propagating through the waist. This interference between modes creates a transmission pattern which depends on the waist length and the effective refractive indexes of the modes travelling through the waist. By applying stress to the tapered fiber its interference pattern can be modified. Chromatic dispersion profile of tapered fibers is obtained, tailored and compared with the dispersion profile of conventional singlemode fibers. 


\section{Resumen}

Esta Tesis se centra en el estudio, implementación y caracterización del control de la dispersión cromática empleando tanto fibra óptica como guías integradas fotónicas. La dispersión cromática provoca que las distintas componentes espectrales asociadas con el pulso óptico viajen a diferentes velocidades. Este efecto puede ser dividido en sus dos contribuciones fundamentales, la dispersión del material y la dispersión de la guía. La dispersión cromática puede ser controlada a través del diseño de los parámetros estructurales del dispositivo para poder obtener así determinadas características en el perfil de dispersión resultante como por ejemplo bajos valores o localización de la longitud de onda de dispersión cero en una longitud de onda deseada. Este método es muy útil en aplicaciones dependientes de la dispersión. En esta Tesis, investigamos el control de la dispersión cromática en dos medios de transmisión diferentes, las guías fotónicas integradas y la fibra óptica.

En el primer caso, se consideran dos geometrías diferentes de guías integradas en silicio, las guías convencionales y las guías ranuradas. Mediante la modificación de los parámetros estructurales como la sección transversal de la guía, su relación de aspecto o el factor de llenado, se obtienen diferentes perfiles de dispersión cromática. Además, se evalúa la influencia de la situación de la ranura. Mediante software de simulación, se obtiene el perfil de índice de refracción efectivo en función de la longitud de onda, que posteriormente se deriva y se obtiene el valor de la dispersión. Asimismo, se mide experimentalmente la dispersión en ambas geometrías utilizando una técnica interferométrica.

En el segundo caso, se analiza la dispersión cromática que presenta una fibra de tipo taper. Esta geometría consiste en una cintura estrecha situada entre dos regiones de transición y permite la modificación de las condiciones de propagación convencionales debido a la interferencia entre los modos que se propagan por la cintura, que crea un patrón de transmisión dependiente de la longitud de la cintura y de los índices efectivos de los modos. Aplicando tensión sobre la fibra, su patrón de interferencia puede ser modificado. La dispersión cromática de las fibras taper se obtiene, se modifica y se compara con el perfil de dispersión de una fibra convencional. 


\section{Resum}

La tesi a exposar se centra en l'estudi, implementació i caracterització del control de la dispersió cromàtica empleant la fibra òptica i les guies integrades fotòniques. La dispersió cromàtica provoca que els distints components espectrals associats amb la pols òptica viatgen a diferents velocitats. Aquest pot dividir-se en les dos contribucions fonamentals corresponents: la dispersió del material i la dispersió de la guia. La dispersió cromàtica pot controlar-se a través del disseny dels paràmetres estructurals del dispositiu per poder obtindre aixi determinades característiques en el perfil de dispersió resultant, com per exemple, baixos valors o localizació de la longitud d'ona de dispersió zero a una longitud d'ona desitjada. No obstant això, aquest mètode és molt útil en aplicacions depenents de la dispersió. A més a més, investiguem el control de dispersió cromàtica en dos mitjans de transmissió diferents, les guies fotòniques integrades i la fibra òptica.

D'una banda, es consideren dos geometries diferents de guies integrades en silici, les guies convencionals i les ranurades. Mitjançant la modificació dels paràmetres estructurals com la secció transversal de la guia, la relació d'apecte o el factor d'ompliment, obtenim diferents perfils de dispersió cromàtica. Fins i tot, s'avalua la influència de la situació de la ranura. Mitjançant el programari de simulació, obtenim el perfil d'índex de refracció efectiu en funció de la longitud d'ona, que posteriorment es derivarà i s'obrindrà el valor de la dispersió. Tanmateix, es mesura experimentalment la dispersió en les dos geometries utilitzant una tècnica interferomètrica.

D'altra banda, analitzam la dispersió cromàtica que presenta una fibra de tipus taper. Aquesta consisteix en una cintura estreta situada entre dos regions de transició que, ens permet la modificació de les condicions de propagació convencional com a causa d'una interferència entre els modes que es propaguen per la cintura i els índex efectius dels modes. Si apliquem tensió sobre la fibra, el seu patró d'interferència podria ser modificat. La dispersió d'una fibra cromàtica de les fibres taper s'obté, es modific i es compara amb el perfil de dispersió d'una fibra convencional. 



\section{List of papers}

The following papers have been derived from the work of the Thesis:

[A] S. Mas, J. Caraquitena, J. V. Galán, P. Sanchis, J. Martí, 'Tailoring the dispersion behavior of silicon nanophotonic slot waveguides', Optics Express, Volume 18, Number 20, 2010. DOI 10.1364/OE.18.020839.

[B] S. Mas, J. Matres, J. Martí, C. J. Oton, 'Accurate chromatic dispersion characterization of photonic integrated circuits', IEEE Photonics Journal, Volume 4, Number 3, 2012. DOI 10.1109/JPHOT.2012.2199294.

[C] S. Mas, J. Palací, and J. Martí, 'Group delay and dispersion tailoring in nonadiabatic tapered fibers', submitted to IEEE Journal of Selected Topics in Quantum Electronics.

[D] S. Mas, J. Palací, P. Pérez-Millán, S. Lechago, D. Monzón-Hernández, and J. Martí, 'All-fiber processing of terahertz-bandwidth signals based on cascaded tapered fibers', Optics Letters, Volume 38, Number 23, 2013. DOI 10.1364/OL.38.004954.

[E] S. Mas, J. Martí, D. Monzón-Hernández, and J. Palací, 'Low-cost refractive index and strain sensor based on tapered fibers', submitted to Optics Communications.

[F] S. Mas, J. Martí, and J. Palací, 'Biconical tapered fibers manipulation for refractive index and strain sensing applications', IEEE Sensors Journal, Volume 15, Number 3, 2015. DOI 10.1109/JSEN.2014.2368146.

[G] S. Mas, J. Martí, and J. Palací, 'Curvature investigation in tapered fibers and its application to sensing and mode conversion', accepted in Optics and Lasers in Engineering.

[H] J. Palací, S. Mas, D. Monzón-Hernández, and J. Martí, 'Nonlinear effects generation in non-adiabatically tapered fibers', submitted to Optical Fiber Technology. 
Other contributions by the author not included in the Thesis:

[I] J. Matres, G.C. Ballesteros, S. Mas, A. Brimont, P. Sanchis, J. Martí, C.J. Oton, 'Optical phase characterization of integrated photonic devices', IEEE Journal of Selected Topics in Quantum Electronics, Volume 20, Number 4, 2014. DOI 10.1109/JSTQE.2013.2292511.

[J] A. Martínez, A. Espinosa-Soria, S. Mas, A. Griol, and F.J. Rodríguez Fortuño, 'Synthesis and measurement of polarization states with silicon nanoantennas', Imagine Nano 2015, Bilbao, España.

[K] S. Mas, J. Palací, R. Caroselli, D. Zurita, J. García-Rupérez, D. MonzónHernández, and J. Martí, 'Refractive index sensor based on a curved biconical tapered fiber', Conferencia Española de Nanofotónica 2014 (CEN2014), Santander, España.

[L] J. Palací, S. Mas, and J. Martí, 'Time domain simulation of optical comb generation in microresonators', Conferencia Española de Nanofotónica 2014 (CEN2014), Santander, España.

[M] L. Sánchez, S. Mas, P. Sanchis, 'Ultra-compact hybrid silicon plasmonic polarization diversity circuit', 10th International Conference on Group IV Photonics 2013 (GFP2013), Seoul, Korea.

[N] S. Mas, J. Matres, J. Martí, C.J. Oton, 'Accurate chromatic dispersion characterization of nanophotonic waveguides with thermal phase noise cancellation', 9th International Conference on Group IV Photonics 2012 (GFP2012), San Diego, USA.

[0] S. Mas, J. Matres, J. Martí, C.J. Oton, 'Accurate dispersion characterization in nanophotonic integrated waveguides', 16th European Conference on Integrated Optics 2012 (ECI02012), Sitges, España.

[P] S. Mas, J. Caraquitena, J. V. Galán, P. Sanchis, J. Martí, 'Dispersion flattening in slot-assisted silicon nanowires', 23rd Anual Meeting of the IEEE Photonics Society 2010, Denver, USA.

[Q] S. Mas, J. Caraquitena, J. V. Galán. P. Sanchis, J. Martí, 'Analysis of Chromatic Dispersion in symmetric and asymmetric silicon slot waveguides', Conferencia Española de Nanofotónica 2010 (CEN2010), Segovia, España.

[R] S. Mas, J. Caraquitena, J. V. Galán, P. Sanchis, J. Martí, 'Tailored Chromatic Dispersion in Silicon-on-Insulator Slot Waveguides', 15th 
European Conference on Integrated Optics 2010 (ECIO2010), Cambridge, United Kingdom. 


\section{Abbreviations used in the text}

AOM

AOPDF

ASE

BPM

CMOS

CMT

DCF

DSF

EAM

EMI

FBG

FDM

FWM

GVD

IR

ISI

LASER

LC-SLM

LD

LED

LPG

MPS

MPI
Acousto-Optic Modulator

Acousto-Optic Programmable Dispersive Filter

Amplified Spontaneous Emission

Beam Propagation Method

Complementary Metal Oxide Semiconductor

Coupled-Mode Theory

Dispersion Compensating Fiber

Dispersion Shifted Fiber

Electro-Absorption Modulator

Electro-Magnetic Interference

Fiber Bragg Grating

Finite Difference Method

Four-Wave Mixing

Group Velocity Dispersion

Infrared

Inter-Symbol Interference

Light Amplification by Stimulated Emission of Radiation

Liquid Crystal Spatial Light Modulator

Laser Diode

Light Emitting Diode

Long Period Grating

Modulation Phase Shift

Multipath Interference 
Optical Network Analyzer

OSA

Optical Spectrum Analyzer

PCF

Photonic Crystal Fiber

QED

Cavity Quantum Electrodynamics

RF

Radio Frequency

SBS

Stimulated Brillouin Scattering

SC

Super Continuum

SNR

Signal to Noise Ratio

SOI

Silicon On Insulator

SPM

Self-Phase Modulation

SPR

Surface Plasmon Resonance

SPW

Surface Plasmon Wave

SRS

Stimulated Raman Scattering

SSFM

Split Step Fourier Method

SSMF

Standard Single Mode Fiber

TE

Transverse Electric

TIR

Total Internal Reflection

TM

Transverse Magnetic

TOD

Third Order Dispersion

TOF

Time of Flight

VIPA

Virtually Imaged Phased Array 
VODL

WDM

WGM

XPM
Variable Optical Delay Line

Wavelength Division Multiplexing

Whispering Gallery Modes

Cross-Phase Modulation 



\section{Chapter 1}

\section{Introduction}

In the mid-twentieth century, several research studies that used optical technology were conducted with the aim of increasing the bitratedistance product of existing microwaves communication systems. However, there were two serious difficulties: there was no appropriate optical source at that time [1] and a suitable transmission medium was required. The appearance of the first Light Amplification by Stimulated Emission of Radiation (LASER) in 1960 provided the ideal method of producing coherent light, that is, the generation of monochromatic waves where all its photons present the same frequency and phase. The next step was to find an adequate transmission medium. To do this, several experiments using atmospheric optical channels were carried out. Nevertheless, this type of channel presented several disadvantages such as limitations due to adverse weather effects or high costs of installation and development. Optical fiber was proposed later as a medium able to confine and guide light. However, the first fibers used for light guiding presented propagation loss values in the range of 1000 
$\mathrm{dB} / \mathrm{km}$ [2]. In 1966, as a result of the studies carried out by Kao and Hockman [3] and Werts [4], where characteristics such as losses, optical mode stability, dispersion and power handling were analyzed, it was found that high propagation losses originated from impurities present in the material used to produce the fibers. Finally, in 1970, researchers at Corning Glass Works managed to reduce losses below $20 \mathrm{~dB} / \mathrm{km}$ in the wavelength region around $1 \mu \mathrm{m}$ [5], laying the fundamentals for the development and evolution of light wave systems.

At a similar time, in 1960, integrated photonics technology was being developed at Bell Laboratories with the fabrication of the first bidimensional waveguide on a planar substrate. In the mid-1970s, several materials were investigated and used to fabricate three-dimensional waveguides. Lithium niobate, $\mathrm{LiNbO}_{3}$, was employed as a prominent material, leading to the development of a variety of functional devices in the 1980s. Further investigations resulted in the development of other materials suitable for the fabrication of integrated waveguides using dielectrics such as glasses, silicon and polymers, or semiconductors such as indium phosphide, gallium arsenide and silicon $[6,7]$.

\subsection{Optical Communication Systems}

The great development experienced by optical communications systems originates from the many advantages that they present with respect to conventional electronic systems. These advantages include a considerable reduction of the weight, cost and size of the wire, huge information transmission capacities, low propagation losses, no need for electrical connections which avoids problems associated with electromagnetic interferences and increased distances between signal repeaters, among others [8]. However, optical communications systems also present some issues that must be addressed, such as curvatures in the optical fiber that modify the propagation conditions of the light introducing losses, the influence of gamma radiation or fragility against animal bites (mainly gophers and termites).

Optical communications make use of very high frequencies typically located in the Infrared (IR), which is the range of the electromagnetic 
spectrum comprising the wavelengths [0.78-1000] $\mu \mathrm{m}$ [9]. Particularly, the systems considered in this work are situated in the Near-Infrared (NIR), located in the wavelength range of [0.78-3] $\mu \mathrm{m}$. These in turn are centered on the C-Band, comprising the wavelength interval of [15301565] $\mathrm{nm}$ [10]. Figure (1.1) shows the electromagnetic spectrum emphasizing the wavelengths used in optical communications systems, specifically the C-Band.

Optical communications systems are made of three main elements: optical transmitters, optical receivers and transmission channels, as shown in Fig. (1.2). The purpose of the system is to transport information from the transmission block to the reception block through the communication channel while avoiding signal loss or distortion [1].

\section{- Optical Transmitter:}

Optical transmitters convert electrical signals into optical ones that can be propagated through an optical transmission channel. The transmitter is formed by an optical source, a modulator and a channel coupler [1], as depicted in Fig. (1.2).

Most optical systems employ one of these two types of semiconductor optical sources: Light Emitting Diodes (LED) and Laser Diodes (LD). They differ in characteristics such as wavelength, optical

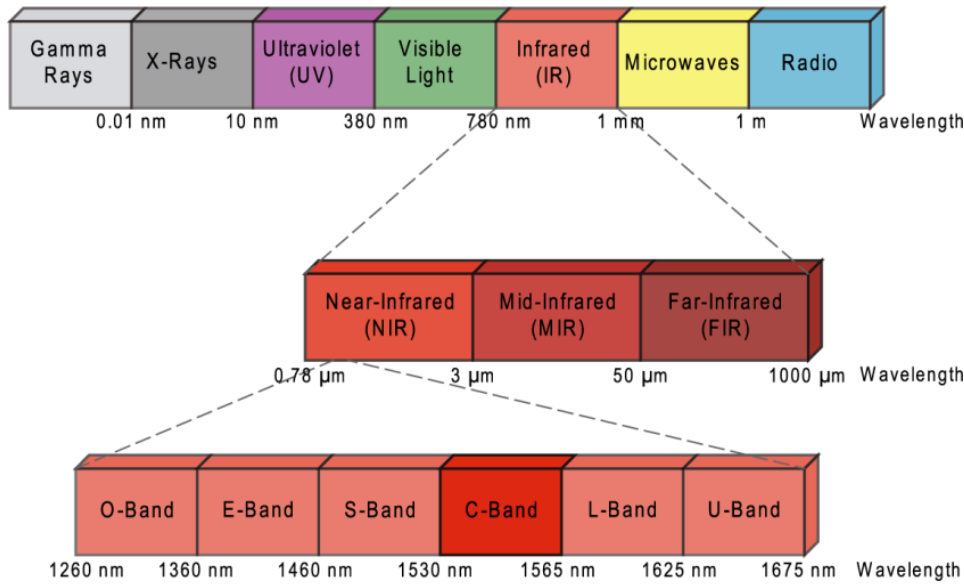

Figure 1.1: Electromagnetic spectrum focused in the C-band. 


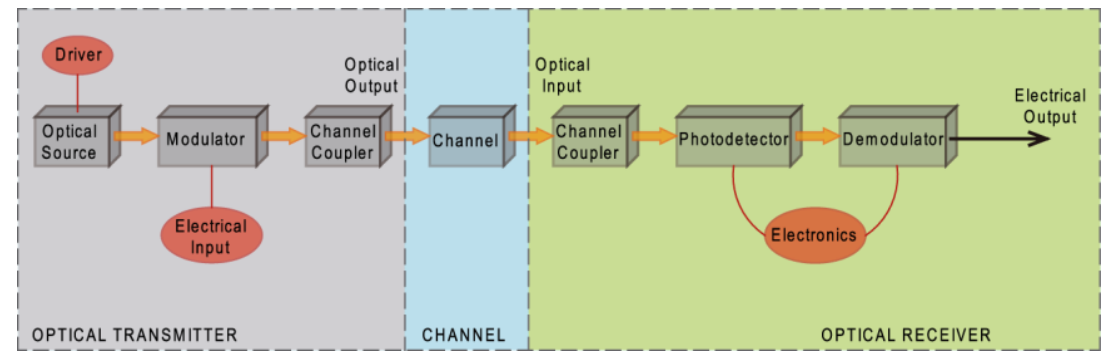

Figure 1.2: Schematic describing an optical communications system.

power, spectral width and output signal waveform. The operating principle is based on the mobility of electrons between the two energy levels of a semiconductor, the valence band and the conduction band. These bands are separated by a space in which there are no energy levels, known as the energy bandgap. When no external field is applied to the semiconductor all the electrons are located in the valence band, which is the one of lowest energy. Through the application of an external electrical field some electrons in the valence band are excited and acquire the energy required to move to the conduction band, leaving holes in the valence band. Conversely, when an electron located in the conduction band loses energy, it returns to the valence band emitting the lost energy as a photon. Recombination between electrons and holes can occur spontaneously, as in the case of LEDs, or stimulated, as in LDs [1, 11]. In the first case, excited electrons of the conduction band fall to the valence band without an external cause, leading to the spontaneous emission of a photon. However, when an existing photon hits an excited electron, a process of stimulated emission occurs, giving rise to coherent light radiation due to the same frequency and phase of the photons involved The three basic processes experienced by electrons in light emission are illustrated in Fig. (1.3).

The purpose of modulation in an optical transmitter is to integrate information in the light beam that will be subsequently launched into the transmission channel. Two types of modulation can be distinguished: direct modulation and external modulation [1, 11]. Direct amplitude modulation involves applying current to a diode above (' 1 ') or below (' 0 ') its emission threshold level, thereby differentiating the bit to be transmitted. This type of modulation is simple and inexpensive but, 
(a)

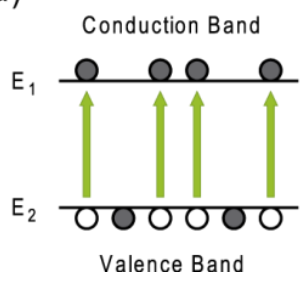

(b)

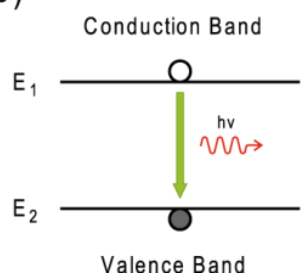

(c)

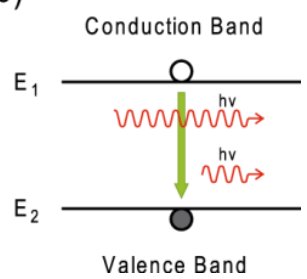

Figure 1.3: Fundamental processes occurring in the two energy bands of an atom, (a) excitation process, (b) spontaneous emission, and (c) stimulated emission.

nevertheless, the output pulses are chirped. The chirp phenomenon causes the frequency and phase of the carrier signal of the transmitted pulses to vary with time, resulting in the widening of its spectrum. External modulation alleviates the effect of the chirp since the modulation is performed by a device which is not part of the optical source. The two most common external modulation devices are the Electro-Absorption (EAM) and the Mach-Zehnder Interferometer (MZI) modulators. In the EAMs, the application of an external voltage decreases the energy bandgap leading to the absorption of light in areas where the device was previously transparent. Meanwhile, in the MZI-based modulators, the application of an external electric field leads to different interference conditions at the interferometer output.

Finally, the last element present in the optical transmitter is a channel coupler, whose function is to couple the maximum amount of signal from the optical modulator to the transmission channel [1]. Micro lens, lensed fibers or fiber tapers, among others, are traditionally employed for channel coupling.

\section{- Optical Receiver:}

The main function of an optical receiver is to convert the optical signals received from the transmission channel into electrical signals that can be read and processed using electronics. The common elements of an optical receiver are a channel coupler, a photodetector and a demodulator [1], as depicted in Fig. (1.2). 
The channel coupler in the optical receiver performs the inverse function of that of the optical transmitter. In this case, it focuses the received signal from the transmission medium onto the photodetector.

Optical-to-electrical conversion is performed on the photodetector or photodiode, which is a device made of semiconductor materials that generates a current, usually referred to as photocurrent, which is proportional to the incident optical power. Photodetectors must meet certain requirements including good sensitivity to the desired wavelength, fast response to be able to operate at high frequencies, low noise to reduce errors, low sensitivity to temperature changes, efficient conversion of photons to electrons and long operating lifetimes $[1,11]$. The three types of photodetectors most widely used in optical receivers are $\mathrm{p}-\mathrm{n}$ junction photodiodes, $\mathrm{p}-\mathrm{i}-\mathrm{n}$ junction photodiodes and avalanche photodiodes, as illustrated in Fig. (1.4). In the first case, the photodetector is formed by a p-n junction separated by an area devoid of free charge carriers. The $\mathrm{p}-\mathrm{i}-\mathrm{n}$ photodiode structure is similar to that of the p-n junction photodiode, with the difference that in this case the depletion area is bigger because an un-doped material layer is inserted therein. Finally, the avalanche photodetector provides an internal current gain, making them suitable for optical receivers in low powered optical links.

The basic operating principle is based on the photoelectric effect, in which an incident photon on the semiconductor material forming the

(a)

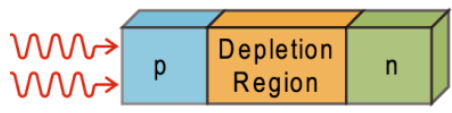

(b)

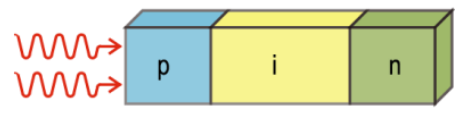

(c)

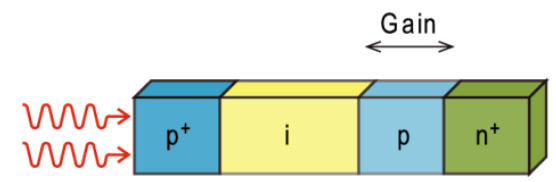

Figure 1.4: Typical configurations of optical photodetectors, (a) p-n photodiode, (b) p-i-n photodiode, and (c) avalanche photodiode. 
photodiode is absorbed by electrons in the valence band, causing the electrons to move to the conduction band. If an external voltage is applied to the semiconductor, the electron-hole pairs generate the photocurrent $I_{p}$, which is directly proportional to the incident optical power $P_{i n}[1]$ :

$$
I_{p}=R \cdot P_{\text {in }}
$$

where $R$ is the responsibility, the parameter which indicates the photocurrent produced by each unit of optical power and is given by the expression:

$$
R=\frac{\eta q}{h v}
$$

where $\eta$ is the quantum efficiency or electron-hole pairs number generated per incident photon with energy given by $h v$ and $q$ is the electron charge. It is worth mentioning that optical receivers often present an amplification step to facilitate the detection of the received signal.

Finally, the last element in the optical receiver is the demodulator, whose operating principle depends on the type of modulation employed. The demodulator extracts the information sent through the optical channel by detecting parameters such as the amplitude and phase of the electrical signal provided by the photodetector and comparing it with a set of thresholds [1].

\section{○ Transmission Mediums:}

The conventional transmission channel of an optical communication system is typically formed by optical fiber. Long distance links usually contain optical amplifiers and regenerators to compensate for losses and distortions introduced by the channel.

The importance of optical fibers as basic elements used in communications systems lies in its many advantages over conventional technologies, such as low transmission losses and huge bandwidth, small size and weight, immunity to electromagnetic interference, electrical isolation, elimination of spark hazards and increased security, among others [1]. 
Optical fiber is a dielectric waveguide able to confine electromagnetic energy and to guide it along its longitudinal axis. The propagation of light inside of the fiber can be described by two different approaches: guided electromagnetic waves called modes or geometrical optics.

Figure (1.5) schematically illustrates light propagation using geometrical optics. This approach considers that a light beam traveling through a medium characterized by a refractive index $n_{1}$ impinges on another medium with a lower refractive index $n_{2}$. The refractive index is a dimensionless parameter that describes the optical properties of a material and is determined by:

$$
n=\frac{c}{v}
$$

where $c$ is the speed of light in vacuum and $v$ is the speed of light in the considered medium. Differences between the two refraction indexes cause a portion of the incident beam to be transmitted to the second medium and another part of the beam to be reflected back into the first

(a)

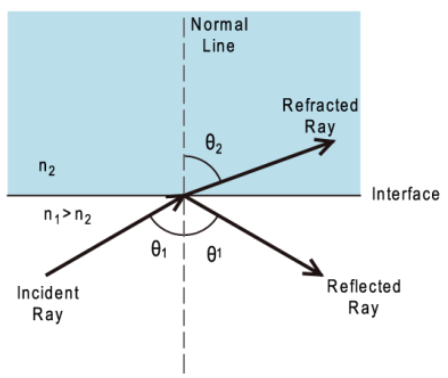

(b)

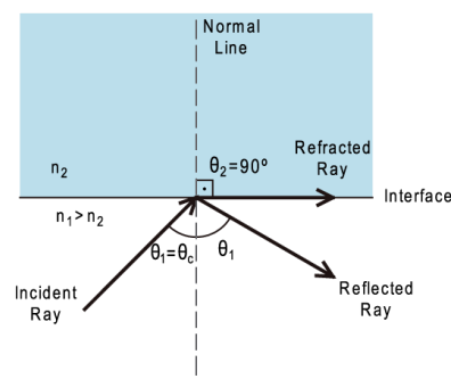

(c)

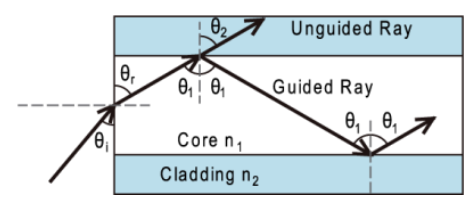

Figure 1.5: Geometrical optics describing the propagation of light, (a) reflection and refraction of an incident ray, (b) total internal reflection (TIR) mechanism, and (c) light propagating through TIR in an optical fiber. 
medium. Snell's law describes the relation between the incident beam angle in the first medium and the refracted beam angle in the second medium $[1,10]$ :

$$
n_{1} \sin \left(\theta_{1}\right)=n_{2} \sin \left(\theta_{2}\right)
$$

where $\theta_{1}$ is the angle formed in the first medium by the incident ray with respect to the normal line and $\theta_{2}$ is the refracted ray angle formed in the second medium also considering the normal line. When the angle of the incident beam exceeds a certain value, known as the critical angle or $\theta_{c}$, the incident ray is completely reflected in the first medium. This phenomenon is known as Total Internal Reflection (TIR). The value of the critical angle is defined by:

$$
\theta_{c}=\arcsin \frac{n_{2}}{n_{1}}
$$

Considering these concepts, and taking into account that the optical fiber is composed of an inner region called core characterized by a refractive index $n_{1}$ higher than that presented by other outer region called cladding, with refractive index $n_{2}$, it can be concluded that light propagates confined through the fiber due to a series of total internal reflections occurring between the core and the cladding $[1,10]$. The scheme of light propagation inside an optical fiber by TIR mechanism is shown in Fig. (1.5.c).

The second description considered here of light propagation in an optical fiber is performed using the mode theory. Modes are the distribution of electric and magnetic fields of the electromagnetic waves through the fiber. The electric and magnetic components of each mode are always orthogonal to each other and both are also orthogonal to the wave propagation direction. Electric and magnetic fields are interrelated and their relationship given by Maxwell's equations. In general, expressions describing electric and magnetic fields are complicated functions in both time and space. However, as the Maxwell equations are linear expressions, the time dependence can be separated from the spatial dependence by substituting those fields by a set of harmonic modes and then taking the real part of them. The harmonic modes of both electric and magnetic fields can be expressed as:

$$
\vec{H}(r, t)=\vec{H}(r) e^{j \omega t}
$$




$$
\vec{E}(r, t)=\vec{E}(r) e^{j \omega t}
$$

where $\omega$ is the angular frequency. The wave equations that are derived from Maxwell's equations for harmonic modes are:

$$
\begin{aligned}
& \nabla^{2} \vec{E}(r)=\varepsilon(r)\left(\frac{\omega}{c}\right)^{2} \vec{E}(r) \\
& \nabla^{2} \vec{H}(r)=\varepsilon(r)\left(\frac{\omega}{c}\right)^{2} \vec{H}(r)
\end{aligned}
$$

In homogeneous mediums, the relative dielectric constant $\varepsilon$ takes a constant value. Thus, the previous equations can be simplified as follows [12]:

$$
\begin{aligned}
& \nabla^{2} \vec{E}(r)+k^{2} \vec{E}(r)=0 \\
& \nabla^{2} \vec{H}(r)+k^{2} \vec{H}(r)=0
\end{aligned}
$$

where $k$ is the wave number, linearly related to $\omega$ according to the following expression:

$$
k=n \frac{\omega}{c}
$$

Equation (1.12) is known as the dispersion relation and is related to the considered homogeneous medium through its refractive index. The general solution of the simplified wave equations for the harmonic waves given by Eq. (1.10) and Eq. (1.11) is a set of plane waves whose simplest solution for the electric field is given by:

$$
\vec{E}(r)=E_{0} e^{j k r}
$$

where $E_{0}$ is a complex value that describes the amplitude and phase of the plane wave. Each of the solutions of Eq. (1.10) and Eq. (1.11), such as the one described in its general form in Eq. (1.13), correspond to different propagating modes for each of the two possible field polarizations, Transverse Electric (TE) and Transverse Magnetic (TM) modes, respectively. Two important parameters that characterize these modes are their cutoff frequency, this is the minimum frequency from which the mode begins to propagate, and its effective refractive index. Basically, the concept of effective refractive index of a mode is related to 
the refractive index of the mode when it is propagating along the waveguiding structure [12].

The number of modes that can propagate within the optical fiber is given by the expression $[1,10]$ :

$$
V=\frac{2 \pi a}{\lambda} \sqrt{n_{1}^{2}-n_{2}^{2}}
$$

where $V$ is the normalized cutoff frequency, $a$ is the core radius and $\lambda$ is the operating wavelength. If $V<2.405$, only one mode propagates inside the fiber while for values exceeding this number several modes travel along the fiber. Optical fibers are classified as single-mode or multimode according to this criterion.

Optical fibers can also be classified according to the refractive index profile along their cross-section. Step-index fibers are those whose refractive index in both core and cladding presents a constant value that does not vary with respect to the radial axis of the fiber, as shown in Fig. (1.6). Step-index multimode fibers experience Multipath Interference (MPI), which leads to signal distortions. In order to reduce MPI in this type of fibers, multimode graded-index fibers were developed, where the refractive index changes gradually along the radial axis, reaching the maximum value at the center of the fiber $[1,10]$.

Otherwise, the rapid advancement of information technology and communications systems made it necessary to develop devices capable of operating at higher speeds with enhanced processing capacities. In this context, integrated photonic technology offers high bandwidth and low power consumption as opposed to electronic technology systems. Many of the optical components currently used consist of a semiconductor material of III-V groups of the periodic table, which implies a higher cost and difficulties in the device integration because assembly and packaging processes are complex and delicate. The successful employment of silicon, $\mathrm{Si}$, as a semiconductor in microelectronic devices has aroused great interest in its possible use in applications oriented to optical communications and signal processing. 

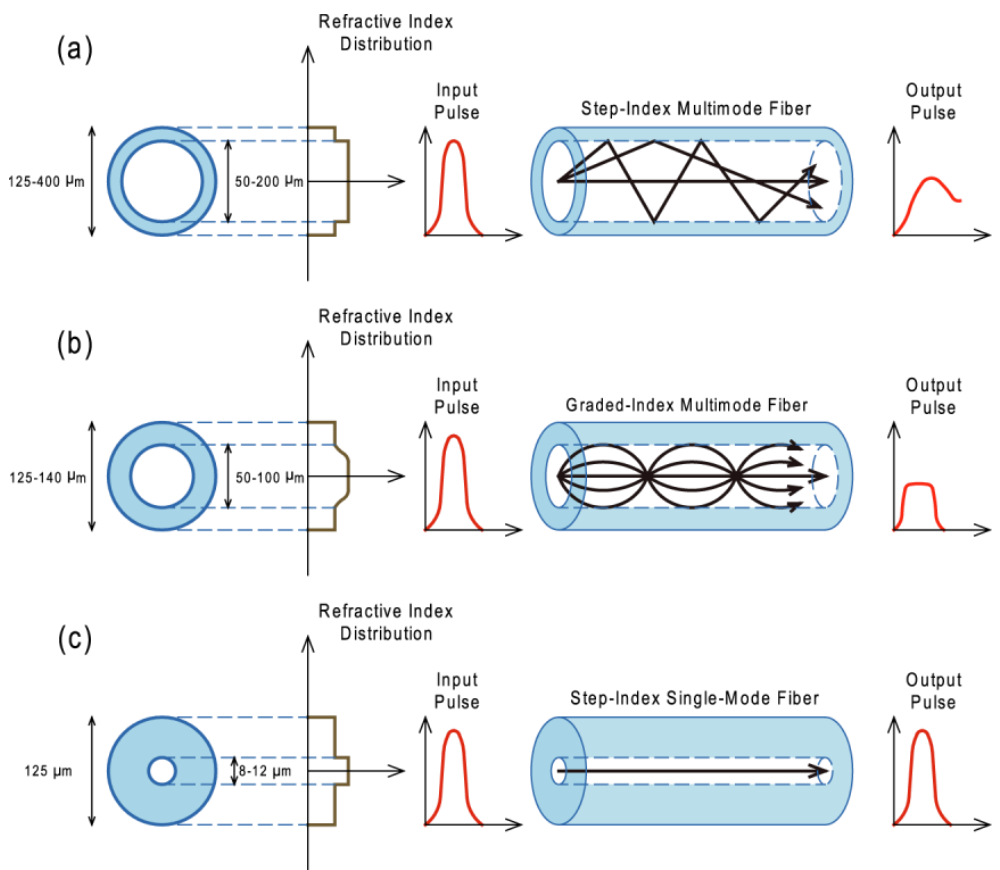

Figure 1.6: Different optical fibers depending on their refractive index distribution and size, (a) step-index multimode fiber, (b) graded-index multimode fiber, and (c) step-index single-mode fiber.

\subsection{Chromatic Dispersion Fundamentals}

Chromatic dispersion is defined as variations in the refractive index of a medium as a function of wavelength. It is usually modeled through the expansion of the propagation constant, $\beta$, in Taylor series around a central frequency $\omega_{0}$ as [13]:

$$
\beta(\omega)=n(\omega) \frac{\omega}{c}=\beta_{0}+\beta_{1}\left(\omega-\omega_{0}\right)+\frac{1}{2} \beta_{2}\left(\omega-\omega_{0}\right)^{2}+\ldots
$$

being

$$
\beta_{m}=\left(\frac{d^{m} \beta}{d \omega^{m}}\right)_{\omega=\omega_{0}}
$$


with $m=0,1,2 \ldots$. The parameters $\beta_{1}$ and $\beta_{2}$ are related to the effective refractive index $n(\omega)$ and its derivatives through the expressions:

$$
\begin{aligned}
& \beta_{1}=\frac{1}{v_{g}}=\frac{n_{g}}{c}=\frac{1}{c}\left(n+\omega \frac{d n}{d \omega}\right) \\
& \beta_{2}=\frac{1}{c}\left(2 \frac{d n}{d \omega}+\omega \frac{d^{2} n}{d \omega^{2}}\right)
\end{aligned}
$$

where $n_{g}$ is the group index and $v_{g}$ is the group velocity, expressed as [13]:

$$
\begin{aligned}
& v_{g}=\left(\frac{d \beta}{d \omega}\right)^{-1} \\
& n_{g}=n+\omega\left(\frac{d n}{d \omega}\right)
\end{aligned}
$$

The coefficient $\beta_{2}$ in Eq. (1.18) is called the Group Velocity Dispersion (GVD) parameter and is the main responsible for pulse broadening in a first order approximation. According to Eq. (1.19), the group velocity is expected to depend on the frequency. Considering a single-mode optical fiber with length $L$, a component of frequency $\omega$ reaches the output end of the fiber after a time delay given by:

$$
\tau_{g}(\lambda)=L / v_{g}(\lambda)
$$

By expressing the group velocity in terms of the propagation constant, the relationship between group velocity and group index can be found:

$$
v_{g}=c / n_{g}
$$

Consequently, the frequency dependence of the group velocity causes pulse spreading since different spectral components do not reach the end of a given fiber reel simultaneously, as shown in Fig. (1.7) where longer wavelengths travel faster. The broadening experienced by the pulse after its propagation through a fiber of length $L$ can be expressed as:

$$
\Delta T=\frac{d T}{d \omega} \Delta \omega=\frac{d}{d \omega}\left(\frac{L}{v_{g}}\right) \Delta \omega=L \frac{d^{2} \beta}{d \omega^{2}} \Delta \omega=L \cdot \beta_{2} \cdot \Delta \omega
$$

where $\Delta \omega$ is the spectral pulse width. In terms of wavelength, the pulse broadening is: 


$$
\Delta T=\frac{d}{d \lambda}\left(\frac{L}{v_{g}}\right) \Delta \lambda=D \cdot L \cdot \Delta \lambda
$$

where

$$
D=\frac{d}{d \lambda}\left(\frac{1}{v_{g}}\right)=\frac{-2 \pi c}{\lambda^{2}} \beta_{2}
$$

is the dispersion parameter, $\mathrm{D}$, which is usually expressed in units of $\mathrm{ps} /\left(\mathrm{nm}^{*} \mathrm{~km}\right)$. The wavelength dependence of $D$ is governed by the frequency dependence of the effective index as follows [13]:

$$
D=\frac{-2 \pi c}{\lambda^{2}} \frac{d}{d \omega}\left(\frac{1}{v_{g}}\right)=\frac{-2 \pi}{\lambda^{2}}\left(2 \frac{d n}{d \omega}+\omega \frac{d^{2} n}{d \omega^{2}}\right)
$$

The dispersion parameter can be expressed as:

$$
D=D_{M}+D_{W}
$$

where $D_{M}$ is the material dispersion and $D_{W}$ is the waveguide dispersion. Material dispersion arises from the dependency of the silicon and silica refractive indices on the optical frequency. It originates from characteristic resonance frequencies at which the material absorbs electromagnetic radiation. Far from these resonances the refractive index $n(\omega)$ can be approximated through the Sellmeier Equation:

$$
n^{2}(\omega)=1+\sum_{j=1}^{M} \frac{A_{j} \cdot \omega_{j}^{2}}{\omega_{j}^{2}-B_{j}^{2}}
$$
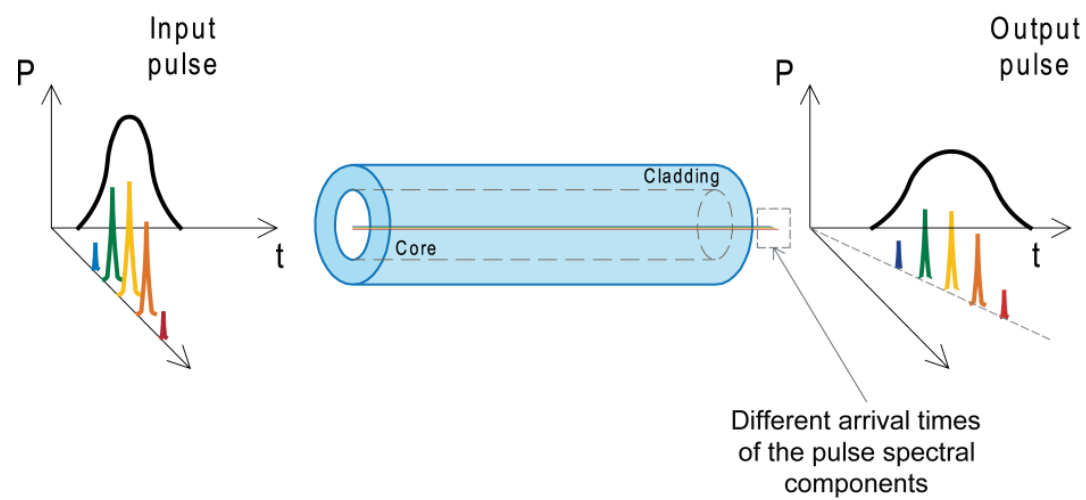

Figure 1.7: Schematic showing the fundamentals of chromatic dispersion and its effect on the time arrival for different wavelengths. 
where $\omega_{j}$ defines the different resonance frequencies and $A_{j}$ and $B_{j}$ are the Sellmeier coefficients. These coefficients are shown in Table 1.1 [14] for silicon and silica. Fig. (1.8) shows the wavelength dependence on the refractive index for both materials extracted from the Sellmeier Equation as well as its material dispersion profile.

Material dispersion relates to the group index slope as follows:

$$
D_{M}=c^{-1}\left(\frac{d n_{g}}{d \lambda}\right)
$$

Moreover, the specific wavelength that satisfies:

$$
\frac{d n_{g}}{d \lambda}=0
$$

is known as the Zero Dispersion wavelength, $\lambda_{Z D}$, as $D_{M}=0$ is obtained when $\lambda=\lambda_{z D}$. The range of wavelengths where $D<0\left(\beta_{2}>0\right)$, is called the Normal Dispersion regime. In the normal dispersion regime, the higher frequency components of the optical pulse travel slower than the lower frequency components. Conversely, for wavelengths that satisfy $D>0$, a situation of Anomalous Dispersion is met in which $\beta_{2}<0$.

Waveguide dispersion depends on the geometry and dimensions of the structure. As known, the mode inside the optical fiber propagates along both core and cladding. The effective refractive index value is therefore between the refractive index of the core and that of the cladding, closer to one or the other depending on the amount of power

\begin{tabular}{|c|c|c|}
\hline \multicolumn{1}{|c|}{ Coefficient } & Silicon (Si) & Silica $\left(\mathrm{SiO}_{2}\right)$ \\
\hline$A 1$ & 10.6684293 & 0.663472443 \\
\hline$A 2$ & 0.00304347484 & 0.440647918 \\
\hline$A 3$ & 1.54133408 & 0.899007061 \\
\hline$B 1$ & 0.301516485 & 0.0665176613 \\
\hline$B 2$ & 1.134755115 & 0.115015076 \\
\hline$B 3$ & 1104 & 9.90316809 \\
\hline
\end{tabular}

Table 1.1: Sellmeier coefficients of Silicon and Silica [14]. 

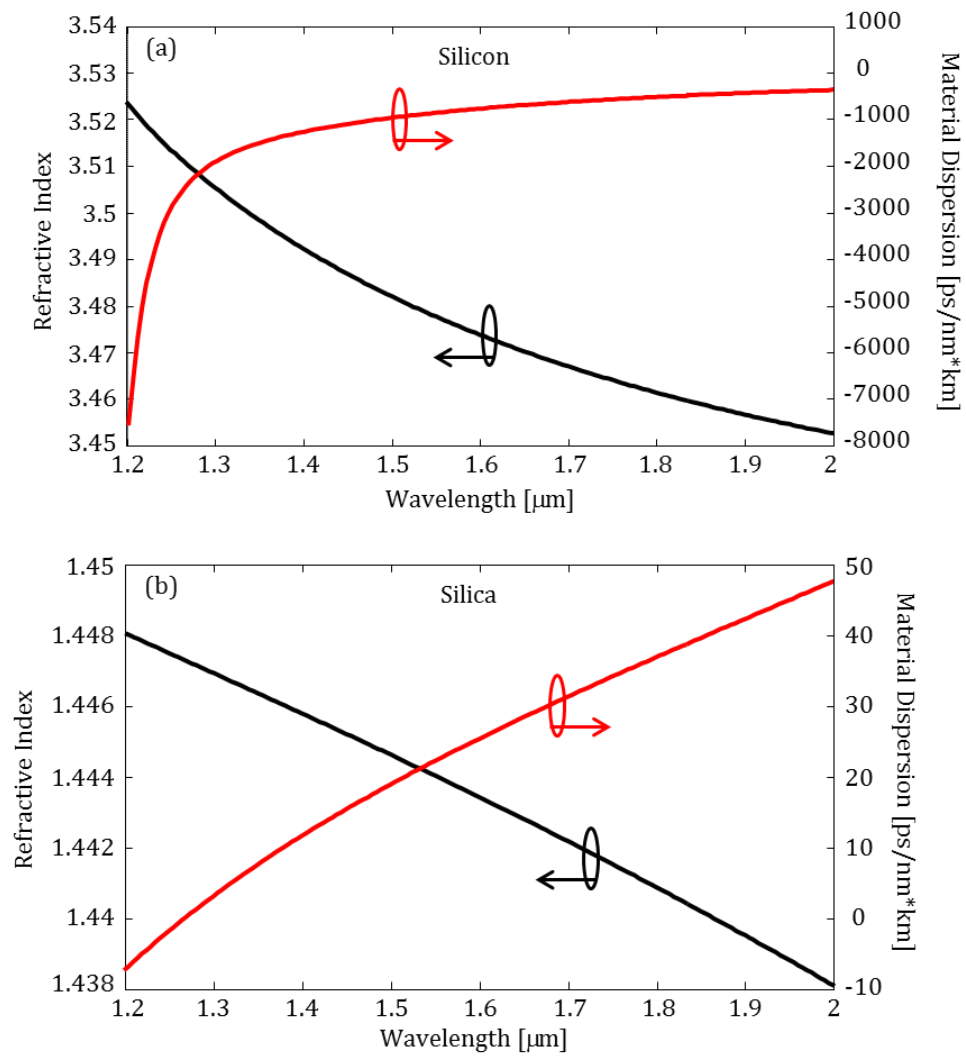

Figure 1.8: Refractive index dependence with wavelength according to the Sellmeier equation and material dispersion profile of (a) silicon and (b) silica.

that propagates through each one (i. e. higher effective refractive indices are expected for highly-confined modes travelling through the core). As the mode power distribution between the core and the cladding depends on the refractive index, which depends on the wavelength, the mode power distribution becomes frequency-dependent. Accordingly, waveguide dispersion exists even for materials with no dispersion at all.

Even at zero dispersion wavelength, the optical pulses experience broadening due to higher order dispersive effects. This feature can be understood by considering that $D$ cannot be zero at all wavelengths contained in the spectrum of the pulse centered at $\lambda_{Z D}$. Although the $\beta_{2}$ contribution is usually the dominant dispersion component, in some 
applications such as transmission of short pulses in high bit rate systems or terahertz spectroscopy it is necessary to include the third order dispersion effects. The higher order dispersive effects are governed by the dispersion slope given by [13]:

$$
S=\frac{d D}{d \lambda}
$$

The $S$ parameter, known as the Differential Dispersion Slope parameter, can be expressed as:

$$
S=\left(\frac{2 \pi c}{\lambda^{2}}\right)^{2} \beta_{3}+\left(\frac{4 \pi c}{\lambda^{3}}\right) \beta_{2}
$$

where

$$
\beta_{3}=\frac{d \beta_{2}}{d \omega} \equiv \frac{d^{3} \beta}{d \omega^{3}}
$$

is the Third Order Dispersion (TOD) parameter. When $\lambda=\lambda_{z D}$ is satisfied, $\beta_{2}=0$ is met and $S$ is proportional to $\beta_{3}$.

Once established the theoretical fundamentals of chromatic dispersion, the next step will be to review the experimental methods related to the measurement of the aforementioned parameters. The techniques capable of measuring chromatic dispersion in optical fibers are mainly three [15]: the Time-Of-Flight (TOF) method, the Modulation Phase Shift (MPS) method and the Interferometry method. TOF and MPS are techniques largely used commercially, unlike Interferometry, whose main use has been found in research [16].

\section{o Time-of-Flight Method (TOF):}

In the TOF technique, the dispersion parameter can be determined by measuring the time delay between pulses at different wavelengths, as shown in Fig (1.9.a), or by measuring the experienced broadening itself $[17,18]$. The relative time delay between pulses at different wavelengths is measured to determine the group velocity, which is subsequently used to obtain the dispersion parameter by means of Eq. (1.24).

The technique involves injecting high power optical pulses into a section of Standard Single Mode Fiber (SSMF), where additional wavelengths are generated due to the nonlinear optical process of Stimulated Raman Scattering (further discussed in Section 3.3.4 of this 
work).The individual wavelengths are selected by an optical filter and then injected into a section of test fiber. Time delays experienced in the test fiber are compared with those obtained in a reference fiber, as shown in Fig. (1.9.b). After that, pulses are photo detected and displayed on a high resolution oscilloscope.

One of the main problems of the TOF technique is that it generally requires several kilometers of fiber to accumulate a significant time delay between the pulses at different wavelengths. Another aspect to be considered in relation to this technique when the pulse broadening is directly observed are the changes experienced in the pulse shape. This leads to variations in the pulse width which results in measurement errors [16].

○ Modulation Phase Shift Method (MPS):

In the MPS technique, a wide spectrum source is modulated by a Radio Frequency (RF) sinusoidal signal, and the dispersion parameter determined by measuring the relative phase shift experienced by the

(a)

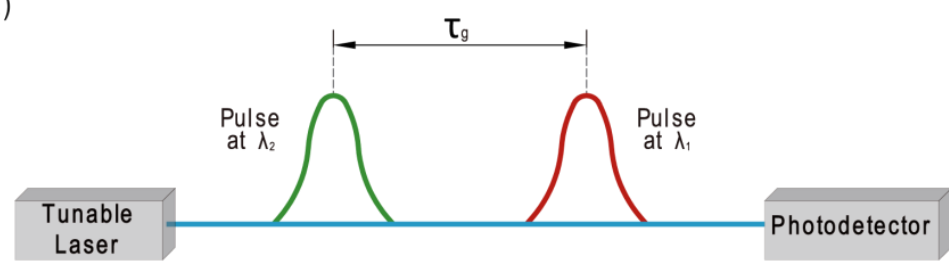

(b)

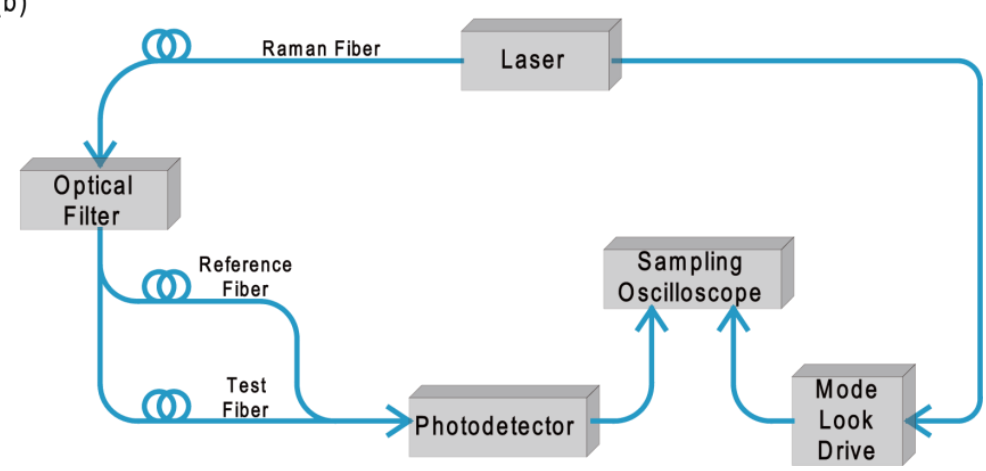

Figure 1.9: Time-Of-Flight method (a) fundamentals and (b) schematic diagram. 
optical carriers at different wavelengths $[19,20]$.The operating principle of the MPS technique is shown in Fig (1.10.a). The phase shift $\phi(\lambda)$ produced is related to the group delay $\tau_{g}(\lambda)$ given by Eq. (1.21) according to the following expression [21]:

$$
\phi(\lambda)=2 \pi f \tau_{g}(\lambda)
$$

where $f$ stands for the frequency of the sinusoidal modulated signal.

Fig (1.10.b) shows the experimental arrangement used to measure dispersion according to this method. A LED source is modulated by the frequency tone provided by a signal generator. The signal is filtered after traveling through the fiber under test and an attenuator placed at the output of the optical filter to compensate for the attenuation of the fiber. Once the signal is photo detected and amplified, a vector voltmeter reads the phase of the output signal relative to the electrically modulated input signal.

The MPS technique is, similarly to TOF, a dispersion measurement method that requires long lengths of fiber. Although it is an expensive

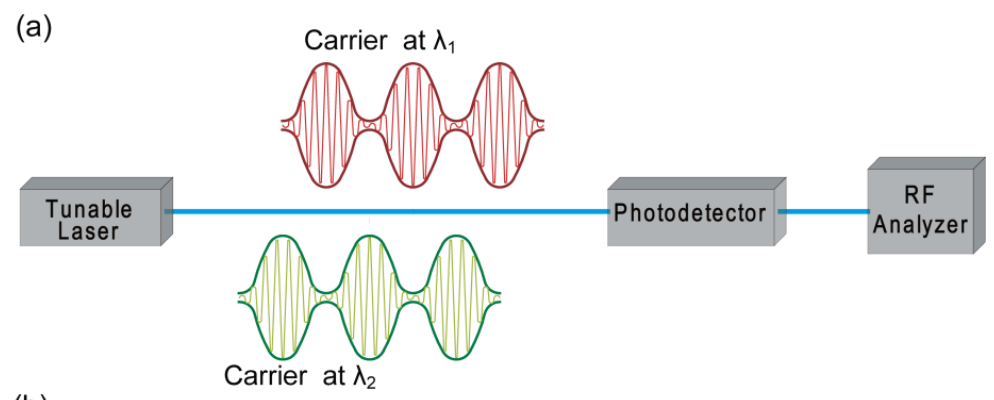

(b)

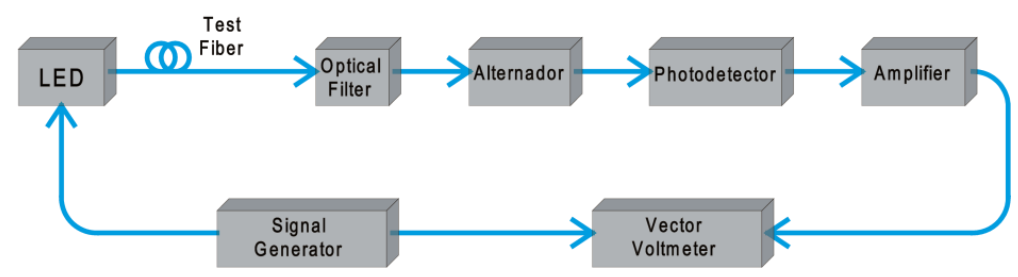

Figure 1.10: Modulation Phase Shift method (a) fundamentals and (b) schematic diagram. 
solution due to the need for RF components it is a reliable and accurate procedure which has become extensively used in the characterization of chromatic dispersion [16].

\section{○ Interferometry Method:}

Interferometry techniques are based on the injection of white light into an interferometer, with one arm being used as the reference path, formed by either a Variable Optical Delay Line (VODL) or an air path, and the other being the fiber or device to be characterized [22, 23]. The different propagation distances cause a phase shift between the signals traveling through the two arms of the interferometer. Once the two signals are recombined at the output of the interferometer, a sine-like interference pattern is generated. The maximum and minimum values in the resulting pattern correspond to constructive and destructive interferences, respectively, and the relative phase value between two adjacent extremes is equal to $\pi$. The value of the group index can be extracted from the resulting interference pattern by using the following expression $[24,25]$ :

$$
n_{g}=\frac{\lambda_{\min } \cdot \lambda_{\max }}{2 \cdot \Delta L \cdot \Delta \lambda}
$$

where $\lambda_{\min }$ and $\lambda_{\max }$ are the spectral positions of a minimum and a maximum in the interference pattern, $\Delta L$ is the length difference between the two arms of the interferometer and $\Delta \lambda$ is the spectral distance between two adjacent extremes.

The fundamentals of the Interferometry technique are illustrated in Fig. (1.11.a), where a fiber-based Michelson interferometer is being used as shown in Fig. (1.11.b). Light emitted by a halogen lamp is chopped to increase the Signal to Noise Ratio (SNR) before being optically filtered. In the all-fiber configuration a single-mode coupler is used instead of a freespace beam splitter, sending part of the signal to the fiber under test and the rest to the VODL. The interference fringes at the output of the lock-in amplifier can be determined by sweeping the length of the reference arm by means of the VODL.

Different methods for the experimental characterization of chromatic dispersion have been developed over the years. Those 
(a)

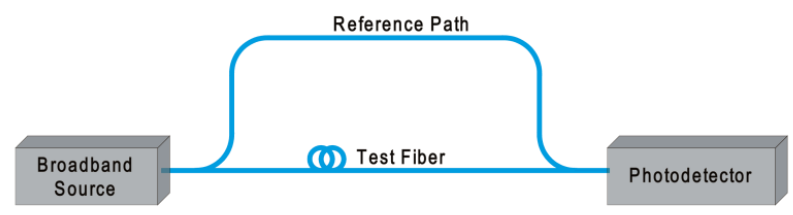

(b)

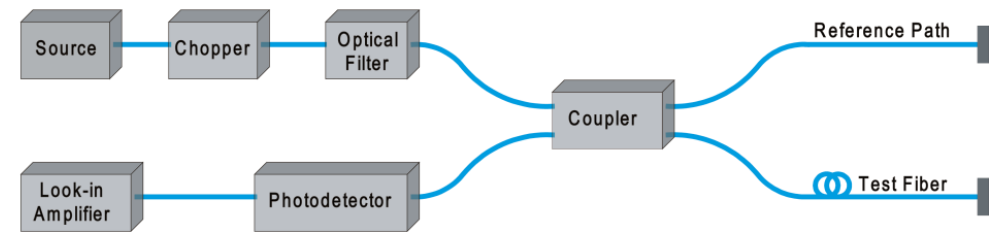

Figure 1.11: Interferometry method (a) fundamentals and (b) schematic diagram.

techniques based on relative frequency shifts or time delays between signals at different wavelengths cannot be employed to determine the chromatic dispersion of short pieces of optical fiber because the relative delay or shift experienced between the signals is not large enough to be resolved. As interferometric techniques provide increased resolution when compared to the previous methods, they can be used to characterize the dispersion not only of short lengths of fiber but also of some photonic integrated circuits.

\subsection{Dispersion Management}

Chromatic dispersion plays a critical role in the propagation of optical signals because it causes diverse spectral components to travel at different speeds [26, 27]. This is especially detrimental in the propagation of wideband signals such as optical pulses, which are broadened thus degrading the quality of the system [28]. For example, dispersion not only increases the noise level in analogical communication systems but can also introduce errors in digital systems. 
In an optical communications digital system, information is transmitted using a codified sequence of pulses whose width is determined by the bit rate $B$ of the system. Considering an optical pulse presenting a Gaussian distribution, the broadening factor can be expressed as [1]:

$$
\frac{\sigma^{2}}{\sigma_{0}^{2}}=1+\left(1+V_{\omega}^{2}\right)\left(\frac{\beta_{2} z}{\sigma_{0}^{2}}\right)^{2}+\frac{1}{2}\left(1+V_{\omega}^{2}\right)^{2}\left(\frac{\beta_{3} z}{4 \sigma_{0}^{3}}\right)^{2}
$$

where $\sigma$ is the width of the pulse, $\sigma_{0}$ is the initial pulse width, $z$ is the propagation direction and $V_{\omega}$ is given by:

$$
V_{\omega}=2 \sigma_{\omega} \sigma_{0}
$$

with $\sigma_{\omega}$ being the spectral width of the source. Assuming that $\beta_{3}$ can be neglected and $V_{\omega}>>1$, the resulting pulse width expression is [1]:

$$
\sigma=\left[\sigma_{0}^{2}+\left(\beta_{2} L \sigma_{\omega}\right)^{2}\right]^{1 / 2}=\left[\sigma_{0}^{2}+\left(D L \sigma_{\lambda}\right)^{2}\right]^{1 / 2}
$$

where $\sigma_{\lambda}$ is the spectral width of the source expressed in wavelength units. An often used relation between $\sigma$ and $B$ can be extracted considering that the broadened pulse must be confined within its own bit slot $T_{B}$. In this case, if the expression $4 \sigma<B$ is satisfied $95 \%$ of the Gaussian pulse energy remains inside the bit slot, leading to:

$$
4 B \sigma<1
$$

Assuming $\sigma_{0}<<$, Eq. (1.39) can be expressed as [1]:

$$
4 B L|D| \sigma_{\lambda}<1
$$

Therefore, signal degradation due to chromatic dispersion depends on the bit rate of the signal and on the modulation used to encode the digital information [29]. In systems operating at low bit rates, dispersion does not produce severe pulse broadening so the pulses can be correctly differentiated at the receiver and the information from each bit properly extracted. However, for higher data rate signals, pulses are shorter and closer to each other. Hence, Inter-Symbol Interference (ISI) may occur making the bit differentiation at the receiver troublesome as illustrated in Fig. (1.12).

From Eq. (1.40), it can be extracted that chromatic dispersion becomes a severe limitation in long-distance high bit rate systems if no 
appropriate acquisition or dispersion compensation techniques are employed. A critical issue related to chromatic dispersion is the appearance of nonlinear effects when the optical communications system operates at the zero dispersion wavelength or around it. Therefore, the aim of dispersion compensation methods is to achieve low average dispersion values on the fiber link as a whole while the dispersion of each section is large enough to avoid the nonlinearities to arise. The dispersion compensation condition can be expressed as:

$$
D_{1} L_{1}+D_{2} L_{2}=0
$$

where $L_{1}$ is the fiber length of the link section considered, $L_{2}$ is the compensation fiber length and $D_{1}$ and $D_{2}$ are its respective dispersion value [1]. This condition can be satisfied by inserting a fiber module which presents the opposite value of the accumulated dispersion of the fiber section considered.

The most common method to implement all-optical compensation for the dispersion of a SSMF is to employ Dispersion Compensating Fiber (DCF) [30]. In these fibers, zero dispersion wavelength is shifted to above $1550 \mathrm{~nm}$ leading to a dispersion value of $-100 \mathrm{ps} / \mathrm{nm}^{*} \mathrm{~km}$ at that wavelength, which makes it capable to compensate for the dispersion of a SSMF [13]. Other types of fibers designed to modify the conventional dispersion characteristics of SSMF include Dispersion-Shifted Fibers

(a)
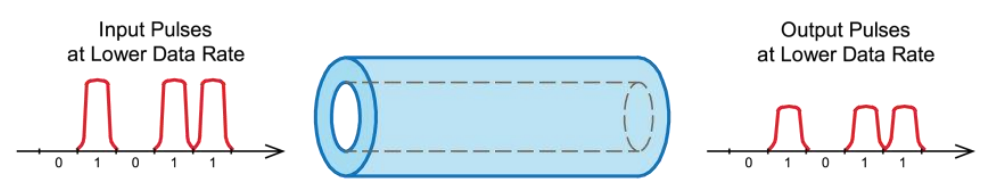

(b)
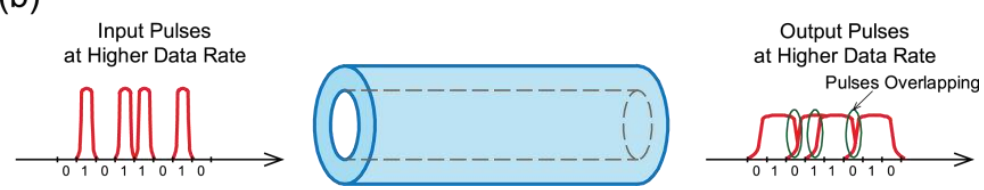

Figure 1.12: Chromatic dispersion effects in a (a) low data rate transmission system and (b) high data rate transmission system. 
(DSF) or Non-Zero Dispersion-Shifted Fibers (NZDSF) [31]. DSF zero dispersion wavelength is located at $1550 \mathrm{~nm}$, resulting in the cancellation of chromatic dispersion but facilitating the arising of nonlinear effects. The use of NZDSF reduces these effects by shifting the zero dispersion wavelength around $1510 \mathrm{~nm}$ and maintaining dispersion level low. Modifications in the dispersion profile of all these different types of fibers are carried out by varying the index difference between the fiber core and cladding and the size of the core diameter [29]. Fig. (1.13) shows refractive index profiles of SSMF, DCF, DSF and NZDSF.

Traditionally, chromatic dispersion compensation in the optical domain is implemented by means of DCF located in different points along the transmission link. However, DCF fibers insert additional attenuation to the system, which makes necessary to insert several amplifiers along the link to compensate for the loss of each section [32]. Therefore, by alternating SSMF sections with compensation modules including DCF and amplifiers, dispersion compensation can be performed in long-distance transmission links. In order to enhance the efficiency of the system, different schemes depending on the placement of the compensation fiber have can be distinguished. In pre-

(a)

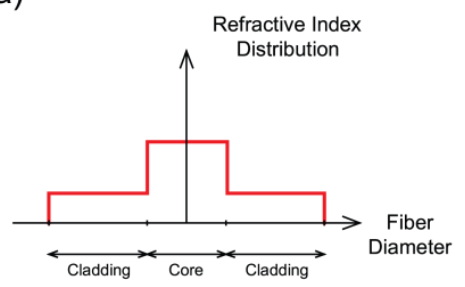

(c)

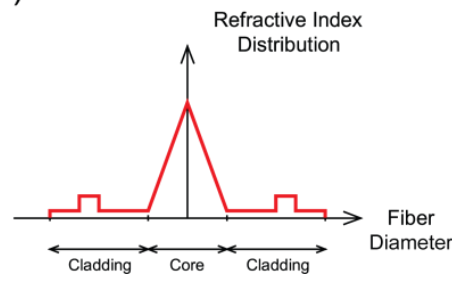

(b)

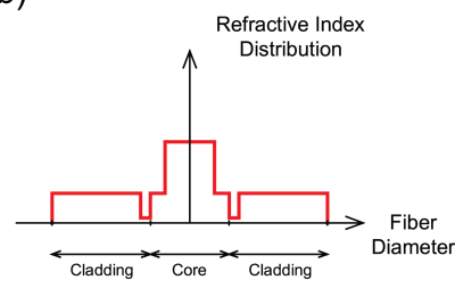

(b)

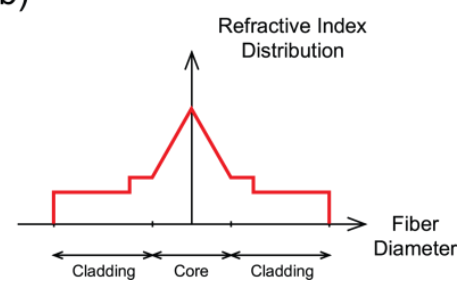

Figure 1.13: Examples of refractive index profile of a (a) Standard Single Mode Fiber(b) Dispersion Compensating Fiber (c) Dispersion-Shifted Fiber and (d) Non-Zero Dispersion-Shifted Fiber. 
compensation schemes, DCF is located before the SSMF to compensate for, while in post-compensation the compensation unit is placed after it. Both arrangements are used at the same time in the pre- and postcompensation scheme [33]. Fig. (1.14) illustrates the arrangements used in all-optical compensation together with the evolution of chromatic dispersion along the distance for each scheme.

In multi-wavelength optical systems, together with chromatic dispersion value management, dispersion slope compensation is required [29]. Dispersion slope is the parameter that represents the quantity of change in chromatic dispersion value per unit of wavelength. If no slope compensation is considered, only the designed wavelength will be correctly compensated, leading to distortions in the transmission over the uncompensated wavelengths. Fig. (1.15) shows the dispersion distribution over the distance with slope compensation and without slope compensation.

Next generation systems operating at higher bit rates will require more advance techniques than the use of DCF due to its losses and cost. In this context, several types of optical adaptive compensators have been developed, employing different structures such as Fiber Bragg Gratings (FBG), Gires-Tournois interferometers or Virtually Imaged Phased Array (VIPA). FBG are reflection-based devices whose response is configured by the modulation of the refractive index of the fiber core. Dispersion management is achieved by applying a voltage to the grating, which results in an effective index variation [34]. In this context, its main characteristics are bandwidth and low losses, together with tuning high resolution and low velocity. VIPA systems employ mirrors and lenses to adjust the optical propagation wavelength, leading to a variation in the angular dispersion [35]. Its characteristics include bandwidth channelbased response, tuning high resolution and moderate velocity and high insertion losses. Finally, Gires-Tournois interferometers present a tunable periodic time delay dependent of the distance between the interferometer mirrors. Dispersion management is carried out by varying the light input angle to the interferometer [36]. This method presents high insertion losses. 
(a)

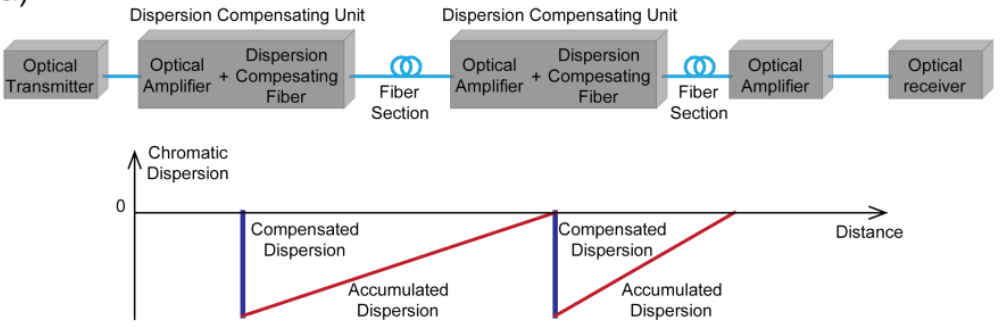

(b)

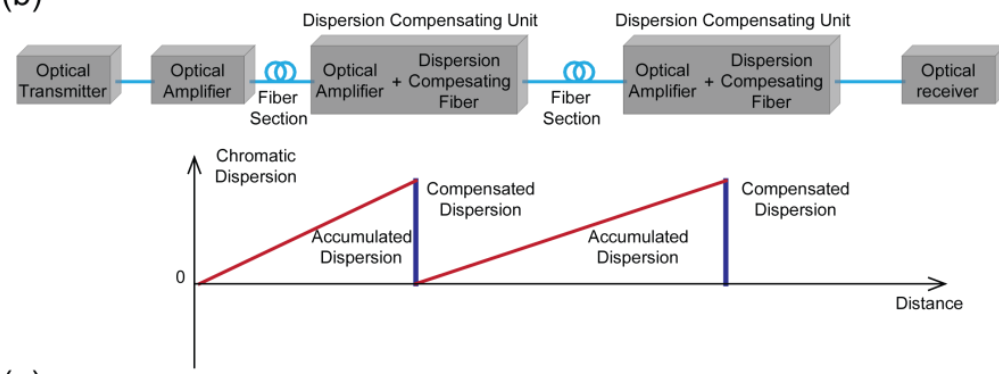

(a)
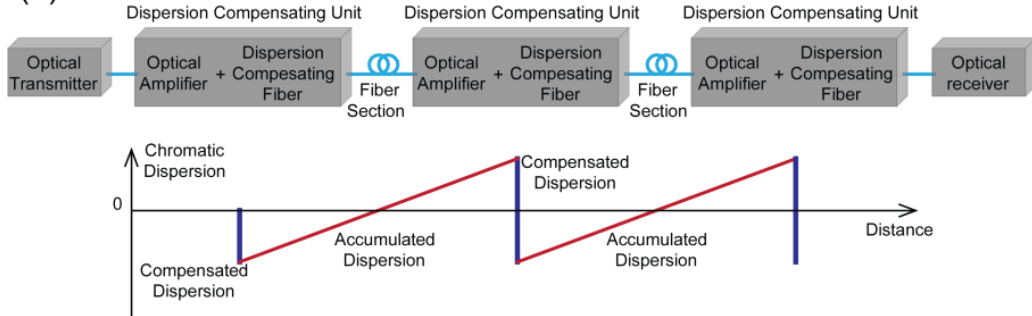

Figure 1.14: Different chromatic dispersion compensation schemes (a) Precompensation (b) Post-compensation and (c) Pre- and Post- compensation.

(a)

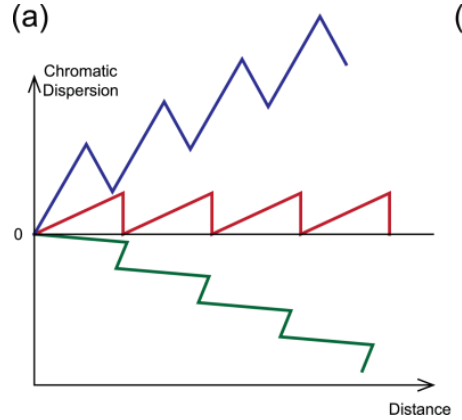

(b)

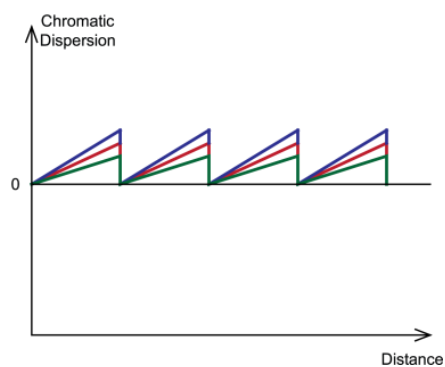

Figure 1.15: Dispersion over distance for (a) dispersion slope compensation and (b) no dispersion slope compensation. 
In the field of silicon photonics, dispersion management consists of the design of waveguide dispersion in order to obtain certain dispersion properties. Therefore, dispersion tailoring is carried out by the appropriate design of the waveguide geometrical parameters such as its thickness $H$, its etch depth $h$ and its width $W[24,37,38]$, as shown in the rib waveguide illustrated in Fig. (1.16). By means of dispersion tailoring of the considered structure, several processes and functionalities can be enhanced, such as nonlinearities [39], soliton generation [40] or broadband optical parametric gain [41], among others.

\subsection{Motivation and Outline}

The objective of this Thesis is to investigate chromatic dispersion tailoring in the two transmission mediums mainly used in optical communications systems, photonic integrated waveguides and optical fibers. In this context, the tailoring is carried out through modifications in the geometry of both structures. In the case of integrated waveguides, different parameters are varied and its effect in the dispersion profile is analyzed. Likewise, dispersion tailoring has been realized in a modification of conventional optical fiber known as tapered fibers bymeans of a stretching process. Furthermore, other functionalities of tapered fibers have been investigated. To do this, results obtained by carrying out several experiments, supported by theoretical calculations when required, are shown.

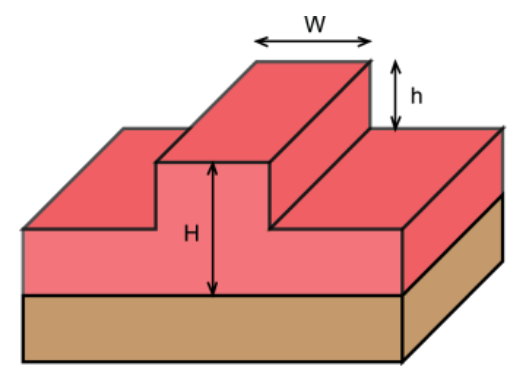

Figure 1.16: Geometrical parameters employed for dispersion tailoring in a SOI rib waveguide. 
According to the university regulations, the format for this thesis is a compilation of articles together with a theoretical introduction to provide the framework of the presented work as well as a discussion of the results obtained. The thesis is organized in five chapters. Chapter 1 has presented the required background in optical communications systems and chromatic dispersion fundamentals, as well as the necessity of dispersion management in order to avoid signal degradation in the transmission medium. Chapter 2 introduces photonic technology and studies chromatic dispersion tailoring in integrated waveguides. In addition, it describes the experimental dispersion measurements carried out in this work for this type of waveguides. Chapter 3 provides an investigation of chromatic dispersion tailoring in tapered fibers, providing both theoretical and experimental analysis. Moreover, different tapered fiber-based experiments showing several functionalities such as pulse shaping, sensing, mode conversion and nonlinearities are presented. Chapter 4 provides a general discussion of the results obtained throughout this thesis and, finally, Chapter 5 summarizes the main conclusions of this work. 


\section{Chapter 2}

\section{Integrated Photonics}

The advantages of using silicon-based optical devices instead of traditional electronics include a potential decrease in size due to the high integration of different opto-electronic components on a single substrate and full compatibility with Complementary Metal Oxide Semiconductor (CMOS) fabrication processes used in the microelectronic industry [42]. Silicon is a dielectric material which is mainly characterized by a high refractive index and an absorption coefficient which is negligible in the wavelength range from $1.11 \mu \mathrm{m}$ to $7 \mu \mathrm{m}$, making it suitable for optical systems in the second and third telecommunication windows. The use of silicon presents, however, some disadvantages such as high propagation losses due to roughness in the walls of the waveguide, low electro-optical coefficient, low light emission efficiency and high losses when carrying out fiber coupling due to large differences between the mode areas [43]. Nevertheless, recent progress in nanofabrication techniques has allowed the creation of a large number of high performance photonic components based on silicon that have successfully overcome their initial limitations as a material for the optical band. 
One of the most developed silicon-based platforms is the Silicon-OnInsulator technology, well established in the microelectronics industry. This technology leverages the high refractive index of silicon, $n=3.47$ at $\lambda=1.55 \mu \mathrm{m}$ [14], and the low refractive index of silica, $\mathrm{SiO}_{2}, \mathrm{n}=1.44$ at $\lambda=1.55 \mu \mathrm{m}$ [14], to introduce a high index contrast and thus favor light propagation and increased integration density. Furthermore, SOI wafers are affordable and show excellent crystalline quality. A conventional SOI wafer comprises a thin silicon layer with a thickness of about $200-250$ $\mathrm{nm}$, deposited on a $\mathrm{SiO}_{2}$ layer of approximately $1-3 \mu \mathrm{m}$ thick whose main function is isolation. They are deposited on a thicker substrate layer of about $700 \mu \mathrm{m}$, also made of silicon that adds mechanical resistance to the structure. Figure (2.1) illustrates the different layers which form a standard SOI structure. Nevertheless, the market offers SOI wafers of a variety of thicknesses and sizes depending on the manufacturer.

One of the main challenges of this technology has been the manufacturing of optical waveguides with low propagation losses. Optical waveguides are used to confine and guide light from one side of the chip to the other and for efficient interconnection among optical devices. In addition, the waveguides may face an optical fiber in case one is required to inject and/or extract light from the integrated circuit. Nevertheless, high light confinement implies higher field intensities on the waveguide walls and, consequently, higher propagation losses due to surface roughness. This issue has already been addressed and low-loss SOI waveguides having propagation loss of $0.3 \mathrm{~dB} / \mathrm{cm}$ are currently available [44]. To sum up, SOI technology makes it possible to

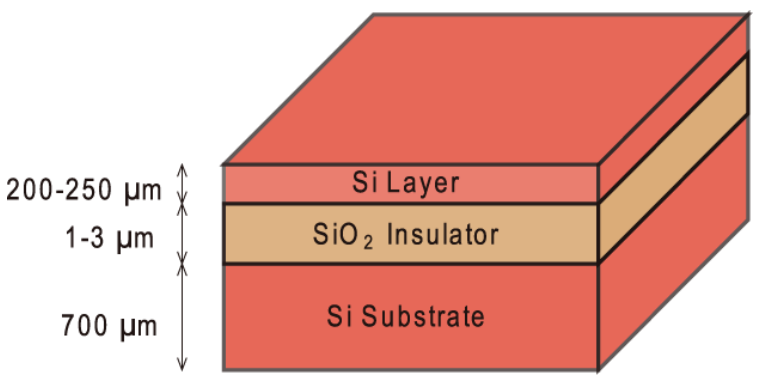

Figure 2.1: SOI wafer diagram showing its different layers. 
manufacture integrated optical waveguides and devices with low losses and high light confinement, enabling the design of high integration density circuits with extremely compact dimensions of the order of nanometers [43].

The propagation characteristics of an optical waveguide mainly depend on its geometry, material properties and light polarization and wavelength. Fig. (2.2) shows some geometries usually employed in the manufacturing of integrated optical waveguides $[27,43]$. In a singlemode waveguide, all of the power traveling through the waveguide is confined in one mode, which is usually desirable in communications systems in order to avoid modal dispersion and obtain higher bandwidth-distance products. The fundamental mode, $\mathrm{TE}_{0}$ or $\mathrm{TM}_{0}$ depending on the dominant polarization, shows no nulls in the entire cross-section of the waveguide, contrary to what happens in higher order modes. Usually, when the waveguide is small enough it presents a single-mode behavior while for increasing dimensions lead to the gradual appearance of higher order modes [24].

As in the case of optical fiber, the analysis of the guided light inside the optical waveguide follows Maxwell's electromagnetic theory. There is

(a)

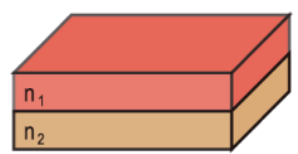

(d)

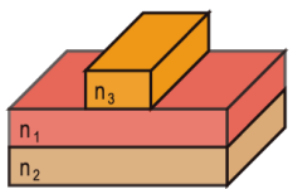

(b)

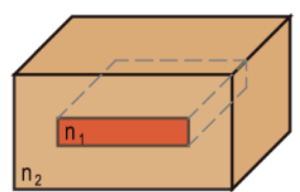

(c)

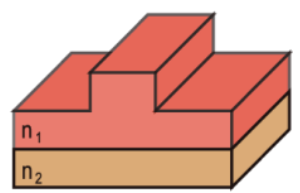

(e)

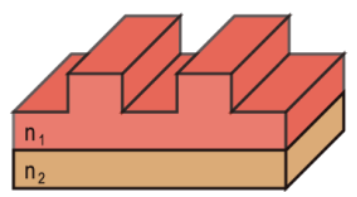

Figure 2.2: Different geometries of SOI integrated waveguides. (a) Planar waveguide, (b) Buried channel waveguide, (c) Rib waveguide, (d) Ridge waveguide, and (e) slot waveguide. 
often no need for a rigorous study of Maxwell's equations and simpler models like geometrical optics can be used. However, the direct modelling and simulation of light propagating through a SOI waveguide becomes difficult when using current software methods due to high computational requirements. One possible solution is the so-called effective index method, which transforms the three-dimensional structure into a two-dimensional model [12] in order to reduce the computational load. Considering that the propagation constant $\beta$ is related to the effective index $n_{\text {eff }}$ according to the expression

$$
\beta=k_{0} n_{\text {eff }}
$$

the method is based on approximating the propagation constants of two- and three-dimensional structures by replacing the refractive index of the waveguide by the effective index of the structure. Taking into account this effective index, it is considered that the propagation constant of the two-dimensional solution is equal to the propagation constant of the original three-dimensional structure. Since the waveguide thickness in the removed dimension is considered in the effective index value no information is lost and accurate results are obtained.

\subsection{Dispersion Tailoring in Integrated Photonics}

Chromatic dispersion in integrated photonic waveguides is investigated in Paper A. Two different geometries are considered, symmetrical and asymmetrical vertical slot waveguides, depending on the location of the slot. The mode propagation along the waveguides is simulated by means of the Beam Propagation Method (BPM) using the commercial Software RSoft [45] and the wavelength dependent effective refractive index of the fundamental mode is extracted. Dispersion profiles in a broad bandwidth centered at $\sim 1.5 \mu \mathrm{m}$ are obtained using Eq. (1.26). Results are analyzed, showing two different dispersion regimes that depend on the geometrical parameters of the waveguides. Moreover, dispersion tailoring is proposed showing that the waveguide dispersion profile can be tuned through design of its geometrical 
parameters. Applications that are influenced in any way by the dispersive properties of photonic integrated waveguides are expected to benefit from this work by using it as a guideline when designing the dimensions of the nanowire. Characteristics such as zero dispersion wavelength location or dispersion profile shape must be designed for applications such as nonlinear generation and ultrashort pulse propagation.

\subsection{Experimental Dispersion Measurements}

Dispersion in integrated optics is often characterized using the Interferometry technique. To do this, a Mach-Zehnder Interferometer configuration is commonly employed [24, 37, 46, 47,48].

Fig.(2.3.) shows two different interferometry configurations used in chromatic dispersion experimental measurements. In Fig. (2.3.a) a basic interferometry diagram can be observed, similar to that used to measure dispersion of short lengths of fiber, wherein the reference path can be constituted by a free-space propagation path or by an arm containing a VODL. In this case, part of the signal emitted by a broadband source is injected into the reference path while the rest is injected and collected from the sample by means of lensed fibers. The state-of-polarization of the light injected into the sample is adjusted using a polarization controller and a polarizer. Finally, the output spectrum is monitored using an Optical Spectrum Analyzer (OSA). Fig. (2.3.b) shows the fundamental blocks of the aforementioned scheme. As in the fiber-based MZI configuration, an OSA allows viewing of the interference pattern.

Chromatic dispersion and group index in silicon nanophotonic waveguides have been experimentally measured in Paper B. Two different waveguide geometries were considered, strip waveguide and slot waveguide. The measurement technique is based on a fiber-based Mach-Zehnder Interferometer wherein a counter-propagating reference beam is introduced. The main advantage of this method lies in the cancellation of the thermal fluctuations present in the system, which provides greater stability to the resulting interference pattern. 
Theoretical calculations for both dispersion and group index are carried out for comparison purposes and found to agree with the experiments. The theoretical chromatic dispersion is obtained by calculating the effective refractive index as a function of wavelength employing the commercial software RSoft and Eq. (1.26), while the theoretical value of the group index is extracted from Eq. (1.20).

(a)

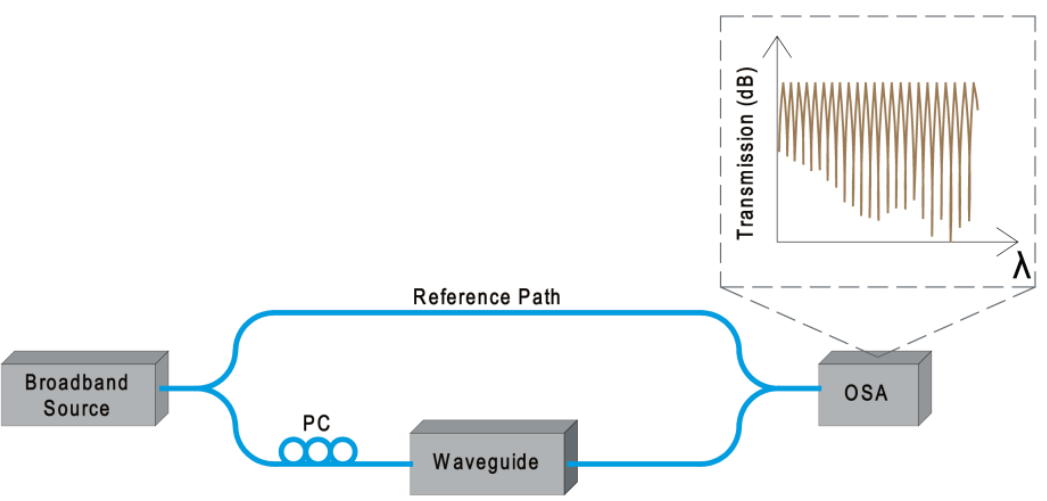

(b)

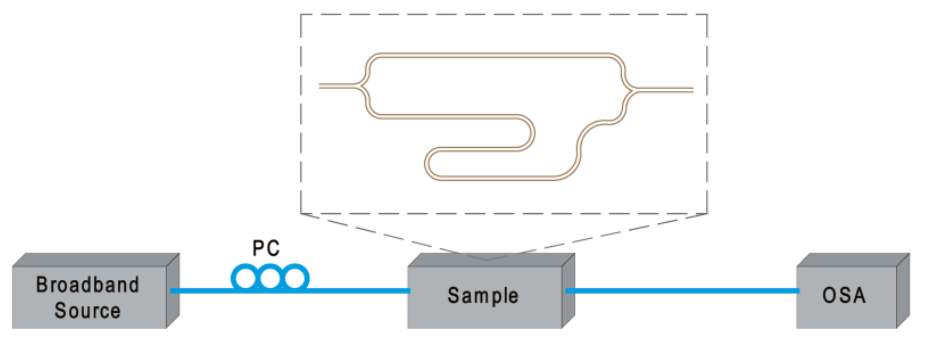

Figure 2.3: Interferometry method in Integrated Optics with (a) fiber-based MZI and (b) integrated MZI. 


\section{Chapter 3}

\section{Tapered Fibers}

Tapered fibers have emerged as a promising device due to their advantageous characteristics, such as relatively low loss, high optical confinement, intense evanescent fields and tunable waveguide dispersion [49]. In addition, the tapered fiber fabrication process is relatively simple, based on stretching and heating a standard singlemode fiber. Its working principle is based on the transformation experienced by the core fundamental mode to a cladding mode in its propagation along the optical tapered fiber [50]. All this has allowed tapered fibers to be widely used in multiple applications.

A tapered fiber is formed by a stretched fiber section, a waist, allocated between two transition regions as shown in Fig. (3.1). Tapered fibers are geometrically characterized by three parameters: taper region length $T_{t}$, waist length $L_{w}$ and waist diameter $\rho$. In addition, the geometric profile of the transition regions must be well determined. Several fabrication processes have been developed in recent years [51]. 


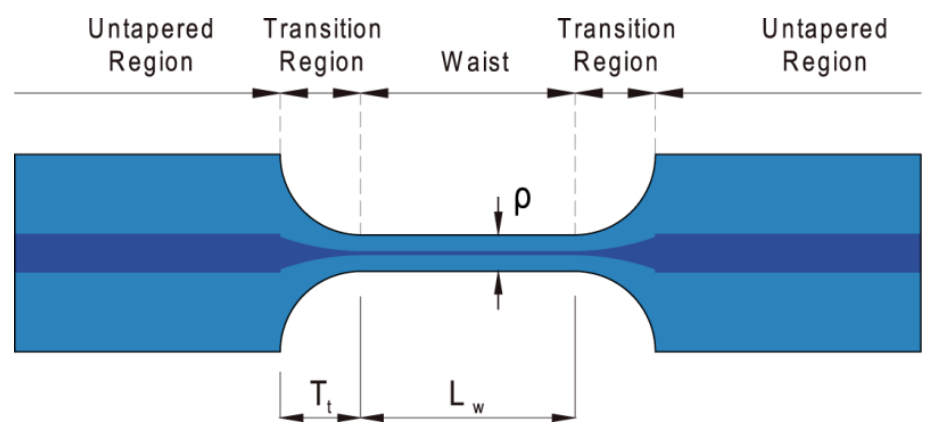

Figure 3.1: Tapered fiber profile with its main geometric parameters.

For example, by introducing the fiber into a micro furnace formed by a sapphire capillary tube heated by a carbon dioxide $\left(\mathrm{CO}_{2}\right)$ laser, clean and reproducible tapered fibers can be obtained [52]. Another method employed is to use an electric stripe heater accurately designed to preserve heat and stabilize the temperature distribution during the manufacturing process [53]. Furthermore, a fabrication technique that reduces the surface corrugations based on the use of a micro-droplet of hydrofluoric acid (HF) for etching the tapered fiber is presented in [54].

However, one of the most popular and simple methods to manufacture tapered fibers is the so-called Flame Brushing Technique $[49,55]$. This technique consists of uniformly heating a fiber section while it is being stretched. For this purpose, a small flame formed by a mixture of butane and oxygen is mounted in a three axis translation stage which allows precise positioning of the flame along with controlled oscillation over a desired distance with constant speed. This distance will determine the waist length of the tapered fiber. Thus, the flame can be accurately placed and displaced, thereby ensuring that the flame heats the fiber uniformly. The fiber coating is removed and the fiber is placed horizontally on a linear stage by two fiber holders. When the flame begins to heat the fiber, the latter is stretched in opposite directions by means of the displacement of the linear stage driven by stepper motors [55]. Moreover, the transmission spectrum of the tapered fiber during the process is monitored using an Amplified Spontaneous Emission (ASE) source and an OSA. Fig. (3.2) shows the experimental setup of the flame brushing technique, which allows the 


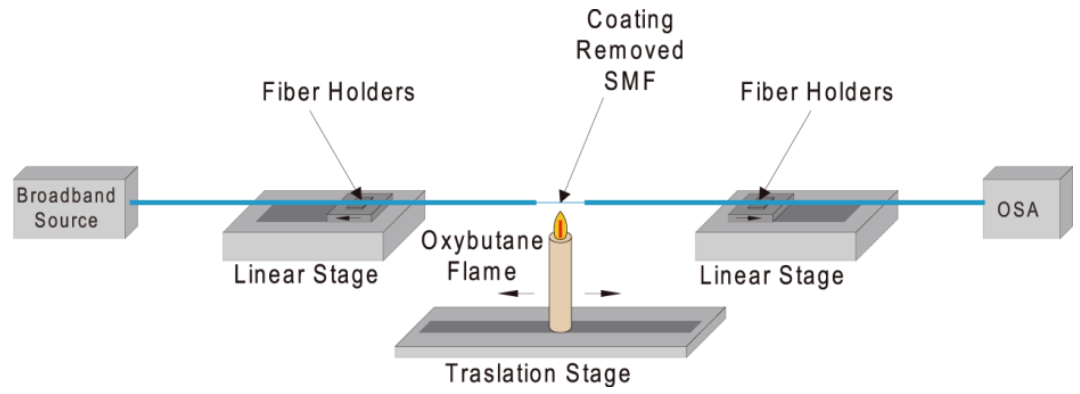

Figure 3.2: Experimental setup of flame brushing technique used in tapered fibers fabrication.

fabrication of tapered fibers with precise length of the waist and constant diameter.

Transition regions can present a multitude of geometric profiles such as linear, exponential or parabolic, among others, depending on several parameters related to the fabrication process. Transition can be represented by a model that relates its shape with the elongation length and the length of the hot-zone, i.e. the fiber segment being heated [49, 50]. In an instant $t$, the equivalent length of the hot-zone delimited by the ends $A$ and $B$ is given by $L$ and the fiber diameter is denoted by $\rho$, as shown in Fig. (3.3). After a short period of time $\delta t$, the heated fiber is subjected to a stretching process in which its length becomes $L+\delta x$ and the value of its diameter becomes $\rho+\delta \rho$. The model is based on the idea of the volume conservation of the fiber before and after the heating and stretching step, so the following expression must be satisfied $[49,50]$ :

$$
\pi(\rho+\delta \rho)^{2}(L+\delta x)=\pi \rho^{2} L
$$

After mathematical operations and integration of Eq. (3.1), the radius profile function is given by:

$$
\rho(x)=\rho_{0} \exp \left(-\frac{1}{2} \int \frac{d x}{L}\right)
$$

where $\rho_{0}$ is the initial radius of the fiber. The tapered fiber profile can be obtained by specifying the shape required for the transition regions. For this purpose, it is necessary to relate the length of the hot-zone with the 
(a)

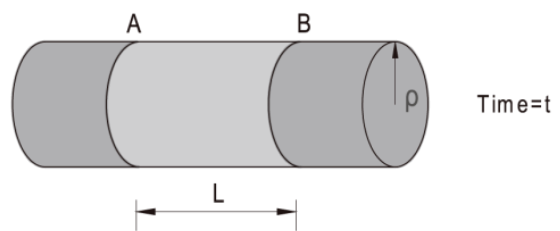

(b)

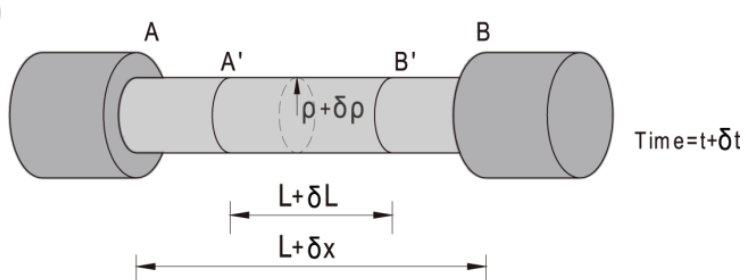

Figure 3.3: Schematic diagrams of a SMF (a) before heating, (b) a short while after heating and stretching.

elongation length during the fabrication process. As an example, a linear function is considered:

$$
L(x)=L_{0}+\alpha x
$$

where $L_{0}$ is the initial length of the hot-zone and $\alpha$ is a constant which can range from -1 to 1 . Then, the expression of the waist diameter is:

$$
\rho(x)=\rho_{0}\left(1+\frac{\alpha x}{L_{0}}\right)^{-1 / 2 \alpha}
$$

As can be gathered from Fig. (3.4), the distances before and after the heating and stretching process satisfy the relationship:

$$
2 z+L=x+L_{0}
$$

Therefore, if we substitute Eq. (3.5) in Eq. (3.4):

$$
\rho(z)=\rho_{0}\left(1+\frac{2 \alpha z}{(1-\alpha) L_{0}}\right)^{-1 / 2 \alpha}
$$

Giving different values to the parameter $\alpha$, different geometric shapes for the transition regions can be obtained. 
(a)

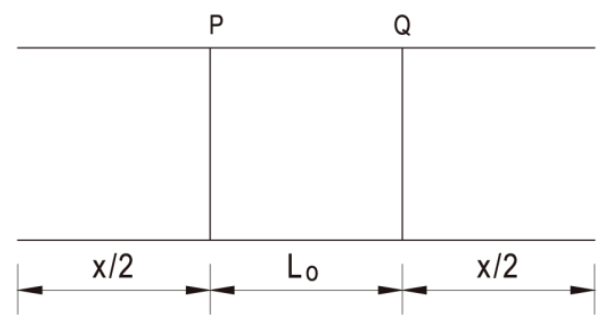

(b)
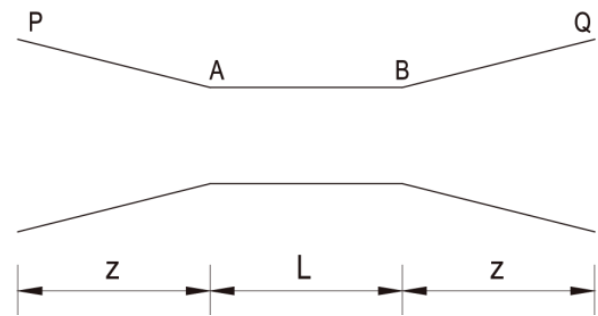

Figure 3.4: Schematic diagrams of the tapered fiber section (a) at the start of tapering and (b) at a time $t$ during tapering.

In the wave propagation along the tapered fiber, the field distribution is modified due to the size differences experienced by core and cladding in the transition regions. If the size reduction is slow enough, most of the propagating power remains in the fundamental mode and suffers no coupling to higher order modes. In this context, it is said that the tapered fiber meets the Adiabaticity criteria [56]. The profile of an adiabatic tapered fiber is shown in Fig. (3.5.a). However, if the transition region presents an abrupt change in the diameter of the fiber, Fig. (3.5.b), mode conversion takes place. As a result, power is transferred from the fundamental core mode $L P_{01}^{\text {core }}$ to the two nearest cladding modes, the fundamental mode $L P_{01}^{\text {cladding }}$ and a higher order mode $L P_{0 m}^{\text {cladding }}$ [57]. These two modes interfere with each other in the output taper due to their different propagation constants giving rise to an interference pattern, as that shown in Fig. (3.6). The phase difference $\Delta \phi$ between these two modes can be expressed as:

$$
\Delta \phi=\frac{2 \pi \Delta n_{e f f} L}{\lambda}
$$


(a)

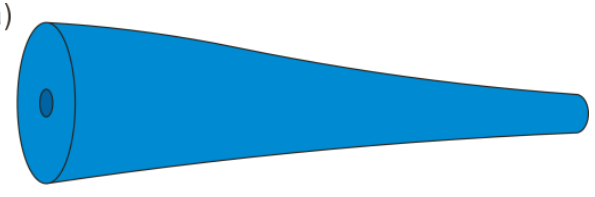

(b)

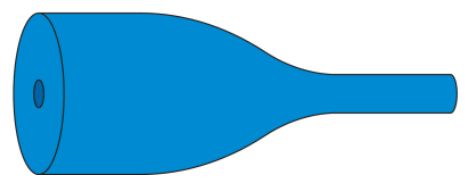

Figure 3.5: (a) Adiabatic and (b) Non-adiabatic tapered fiber.

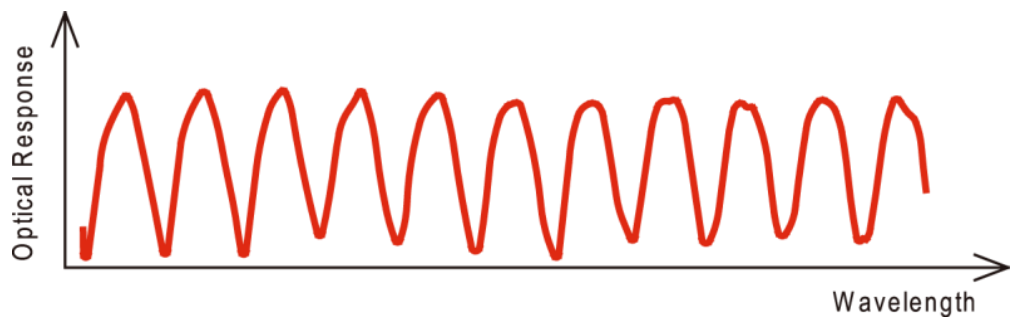

Figure 3.6: Transference function of a Non-Adiabatic Tapered Fiber.

where $\Delta n_{\text {eff }}$ is the effective refractive index difference between the two propagating modes, $L$ is the interference length and $\lambda$ is the operating wavelength. Therefore, by varying any of the parameters involved in Eq. (3.7), the spectral response of the tapered fiber can be modified. When higher order modes reach the waist, they cannot be guided inside the fiber anymore and they are diffracted as radiating modes. Furthermore, when the refractive index of the external medium is higher than that of the cladding, the mode is lost by scattering and absorption [57]. Subsequently, in the output transition region, the beating modes power is recombined in the core fundamental mode $[49,51,58]$. 


\subsection{Dispersion Tailoring in Tapered Fibers}

Tapered fibers are widely employed in many applications. An example can be found in the use of tapered fibers in dispersion compensation. This is due to the fact that it presents a large anomalous dispersion value in the waist that can compensate for normal dispersion in the system. Dispersion tailoring in tapered fibers is therefore possible by means of the variation of the effective indexes difference of the modes propagating along the waist, on one hand, and the waist shape on the other hand, as can be extracted from Eq. (3.7).

A common way to introduce changes in the effective indexes difference is to modify the refractive index of the surrounding medium. In this context, the dispersion characteristics of the tapered fiber can be tailored by immersing the structure in different liquids. Dispersion tailoring in different fluids such as acetonitrile, pentane and hexane [59] or even heavy water [60] has been demonstrated.

Regarding to the waist shape, dispersion engineering can be carried out by modifying the waist geometrical parameters, such as its length and diameter. Initially, when considering single mode fibers with diameter values of $125 \mu \mathrm{m}$, tapered fibers present normal dispersion. However, as waist diameter decreases in the tapering process, the amount of anomalous dispersion in the tapered fiber increases. However, it is important to note that loss increase with the waist reduction, thus a careful design taking into account these considerations is required [61]. Furthermore, the zero dispersion wavelength of the tapered fiber is shifted to shorter wavelengths when the waist is decreased, being able to present even a second zero dispersion wavelength [62]. Also, the pump wavelength influence must be considered when designing the desired dispersion profile [63]. Finally, dispersion engineering by variations in the taper length is analyzed in the next paper presented in this work and explained in the next paragraph. 


\subsection{Experimental Dispersion}

\section{Measurements}

Chromatic dispersion tailoring in a nonadiabatic tapered fiber is experimentally obtained and analyzed in Paper $\boldsymbol{C}$. First, by using an Optical Network Analyzer (ONA), transmission response, group delay and chromatic dispersion in function of wavelength are acquired for a single-mode fiber and a tapered fiber with the same length and characteristics. Results for both fibers are compared in order to analyze the influence of the fiber taper. Subsequently, dispersion tailoring is carried out in the tapered fiber by stretching it into three different points of elongation. As known, the transmission response of the tapered fiber depends on the waist length and the effective refractive indexes of the propagating modes. Therefore, by stretching the tapered fiber, its propagation characteristics can be modified in a dynamic and very simple way leading to the fine tuning of the resulting dispersion profile. Furthermore, coarse dispersion tailoring is obtained by the modification of the surrounding medium of the tapered fiber, which results in local maximum dispersion values of $700 \mathrm{ps} / \mathrm{nm} * \mathrm{~km}$, more than 40 times the conventional dispersion value of $17 \mathrm{ps} / \mathrm{nm} * \mathrm{~km}$ at $1550 \mathrm{~nm}$ present in a SSMF.

\subsection{Tapered Fiber Applications}

Tapered fibers can be used in the generation of the so-called effect SuperContinuun (SC), based on the nonlinear spectral broadening experienced by the pulses of a narrow band source, resulting in a spectrally continuous output response in a very wide bandwidth [64]. SC generation is a nonlinear process which depends on several parameters such as the fiber length, the dispersion profile, nonlinearities generated or the input pump wavelength, among others. In addition to the possibility of easily tailor the dispersion profile and shift the zero dispersion wavelength, the use of tapered fibers in supercontinuum generation presents the advantage of not affecting the coherence of the generated supercontinuum spectrum, as can happen when using other devices such as PCF for this purpose [62, 65]. 
Another application where tapered fibers can be satisfactorily used is in the optical power coupling to microresonators. Microresonators are very interesting devices that are widely utilized in processes such as filtering, sensing, nonlinear optics or Cavity Quantum Electrodynamics (QED), among many others [66]. In order to carry out the optical coupling, the propagating constant of the mode travelling through the coupling structure must match the propagation constant of the desired mode inside the cavity. Besides the use of tapered fibers, other two main methodologies are developed for light coupling into and out of the microresonator based on prisms and side-polished fibers, respectively. In the former case, the evanescent field present in the surface of the prism is composed of several free-space modes which are coupled to the desired mode in the cavity. In the case of the side-polished fibers, the propagation constant of the mode traveling through the fiber is much more similar to the mode inside the cavity than in the case of prism coupling. However, this method is limited by the size of the microresonator [67]. The use of tapered fibers for light coupling into and out of microresonators is shown to have a higher coupling efficiency than the previous two methods, as well as lower insertion loss.

Together with the already mentioned applications, tapered fibers can be employed in many others. Among them, processes such as pulse shaping, sensing, mode conversion and nonlinear generation will be described a little more in depth in the following sections of this work.

\subsubsection{Pulse Shaping}

In some contexts, the modification of different characteristics of the optical pulse such as amplitude, phase and polarization state is required. The set of techniques that allows the generation of nearly any user defined pulses is called Pulse Shaping [68, 69]. In this work, the Fourier transform pulse shaping is considered, as it is the most commonly used technique for pulse shaping purposes. To this end, an optical pulse defined by functions in time and frequency domains denoted $e_{i n}(t)$ and $E_{\text {in }}(\omega)$, respectively, passes through a pulse shaper which presents a spatial mask $M(x)$, as can be seen in Fig. (3.7). The transference function

of the pulse shaper is a complex function representing both intensity and 
phase filtering given by $M(\alpha \omega)$, where $\alpha$ is the spatial dispersion at the masking plane [68]:

$$
\alpha=\frac{\partial x}{\partial \omega}
$$

Therefore, the field at the output of the pulse shaper is given by multiplying the spectrum of the input pulse by the spectral response of the pulse shaper as follows:

$$
E_{\text {out }}(\omega)=M(\alpha \omega) \cdot E_{\text {in }}(\omega)
$$

In order to obtain the field at the output of the pulse shaper in the time domain, the inverse Fourier transformation of $E_{\text {out }}(\omega)$ must be performed:

$$
e_{\text {out }}(t)=e_{\text {in }}(t) * m(t / \alpha)
$$

where

$$
m(t / \alpha)=\frac{1}{2 \pi} \int M(\alpha \omega) e^{j \omega t} d \omega
$$

is the spectral resolution impulse response function. As shown in Fig. (3.7), a signal function of both space and time is obtained at the pulse shaper output. This is due to spatial variations and diffraction in the mask, that lead to different frequencies leaking in different locations of the mask and experiencing different spatial reshaping. Therefore, the output signal of the pulse shaper must traverse a spatial filter to remove this dependency. Whereas the spatial filter isolates a Gaussian spatial mode, the output field expressions in the time and frequency domains are given by [68]:

$$
\begin{aligned}
& E_{\text {shaped }}(\omega) \sim\left[\int d x M(x) \exp \left[-2(x-\alpha \omega)^{2} / w_{0}^{2}\right]\right] E_{\text {in }}(\omega) \\
& e_{\text {shaped }}(t) \sim e_{\text {in }}(t) *\left[m(t / \alpha) \exp \left(-w_{0}^{2} t^{2} / 8 \alpha^{2}\right)\right]
\end{aligned}
$$

where $w_{0}$ is the Gaussian field radius.

The operation principle of a pulse shaper based on optical Fourier transformations between time and frequency domains is shown in Fig. (3.8). First, a decomposition of the incident pulse into its spectral 


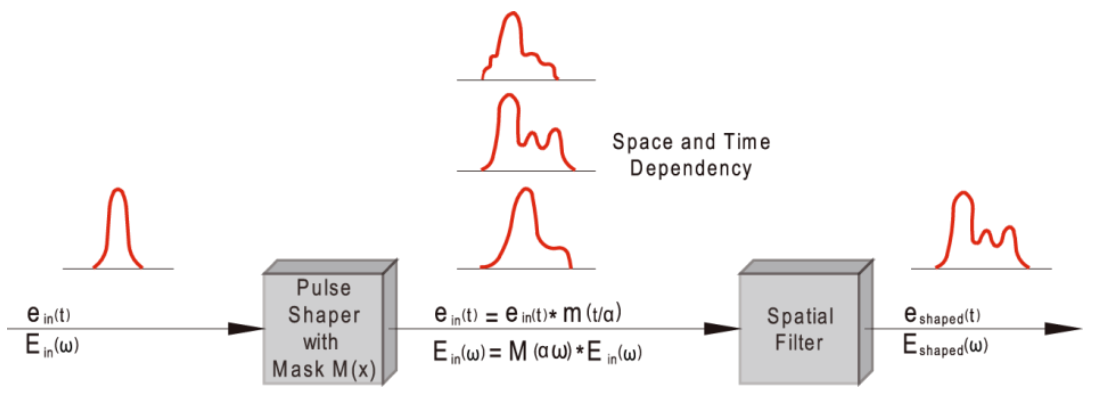

Figure 3.7: Operation principle of pulse shaping.

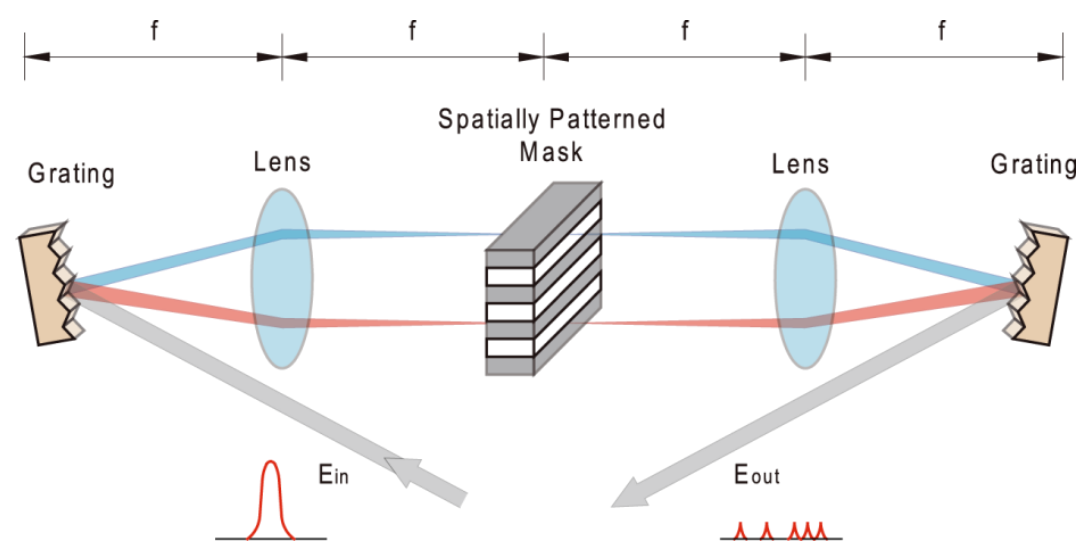

Figure 3.8: Schematic of $4 f$ Fourier-transform pulse shaping arrangement.

components is conducted by a spectral disperser, such as a grating. Then, the spectral components are focused by a lens of focal length $f$. The spectral components are modulated as they pass through the spatially patterned mask. Subsequently, these components are recombined by means of a focusing element and a spectral disperser. Finally, the optical pulse is reconstructed using a mirrored setup consisting of an identical focusing element and spectral disperser, resulting in an output pulse whose shape is the Fourier transform of the pattern transferred from the mask to the frequency spectra of the input pulse. This configuration, without the mask, is called $4 f$ pulse shaping arrangement because it introduces no temporal dispersion if the $4 f$ condition is met $[68,69]$.

The ability of a pulse shaper to generate a wide range of different waveforms is called programmability. The most popular arrangements of 
programmable Fourier transform pulse shapers are based on the use of different types of modulators to conform the mask. Fig. (3.9) shows three typical configurations of modulator-based pulse shapers: Liquid Crystal Spatial Light Modulator (LC-SLM), Acousto-Optic Modulator (AOM) and Acousto-Optic Programmable Dispersive Filters (AOPDF) $[68,69]$.

LC-SLMs rest on the spatial dispersion and modification of the refractive index value of the wavelength components of the incident pulse inside the pulse shaper [70]. The refractive index variation is carried out by applying voltages to the electrodes of the modulator, resulting in a delay of some wavelength components over others. LC-SLM is a widely used device in phase-only pulse shaping. As seen in Fig. (3.9.a), LC-SLM is formed by a thin region of a liquid crystal material located between two glass plates. In turn, the liquid crystal material is formed by a set of long and thin molecules that do not present translational order but have orientation order. When no voltage is applied to the modulator, the alignment of the molecules along the $y$ direction provides a higher refractive index to the light polarized in that direction than that of the light polarized along the $x$ direction. Conversely, when an electric field is applied to the LC-SLM, the molecules tilt along the longitudinal direction $z$, modifying the phase of the light polarized along the $y$ direction.

AOM pulse shapers present a similar configuration to the $4 f$ Fouriertransform pulse shaping depicted in Fig. (3.8). However, in the AOM arrangement the grating and lens are positioned displaced to account for the beam deflection caused by the acousto-optical modulation, as can be seen in Fig. (3.9.b). An acousto-optic modulator fed by a RF arbitrary waveform generator is used as mask generator [71]. In the modulator, the RF electrical signal drives a piezoelectric transducer, which generates an acoustic wave. Modulation of the incident pulse in the pulse shaper occurs due to the diffraction experienced by the light due to the refractive index changes that the acoustic wave produces during its propagation. Programmability in this type of pulse shaper is achieved if the RF signal is modulated, as the acoustic wave profile is a scaled and delayed version of the RF signal.

Fig. (3.9.c) shows the simplified operation principle of the third type of pulse shaper considered in this work, AOPDF pulse shapers [72]. As in the AOM pulse shapers, a programmable RF signal drives the 
piezoelectric transducer creating an acoustic wave which replicates spatially the temporal shape of the RF signal. The incident pulse in the AOPDF propagates with certain polarization along one of the major axes of a photo elastic crystal until it encounters a crossed polarizer that extinguishes the signal. This occurs when the incident pulse suffers no interaction along its propagation through the AOPDF. However, if an acoustic wave propagating collinearly with the incident pulse is launched, the interaction of the two signals causes a change in the birefringence which leads to a light coupling from the input polarization to its orthogonal polarization. In this latter state of polarization, the signal can propagate through the crossed polarizer. If the RF signal is a single tone, the described arrangement results in an optical band pass filter whose center frequency can be tuned by varying the RF signal frequency.

Due to the large number of different waveforms that can be obtained [73], pulse shaping technique for ultrashort pulses is widely used in many applications such as spectroscopy, microscopy, signal processing, dispersion compensation, nonlinear optics, spectral phase encoding and decoding, filtering and biomedical imaging, among others [68, 74]. Although pulse shaping methods based on Fourier transform are the most widely used, some other pulse shaping alternatives have been developed by means of nonlinear frequency mixing [75] or LFBG [76], among others. In fact, a pulse shaper can be considered as a filter which can emulate any linear combination of pulses and, therefore, devices such as Michelson or Mach-Zender interferometers can be contemplated as pulse shapers too. Thus, devices such as tapered fibers can be also considered for pulse shaping applications, as their operating principle is similar to that of a MZI.

A method for femtosecond pulse shaping by using tapered fibers is proposed and demonstrated in Paper $\boldsymbol{D}$. An in-line combination consisting of one fixed and one tunable tapered fiber is considered. By introducing a stretching in the tunable tapered fiber, the optical response of the combination experiences a spectral shift due to the variation in the interference conditions between the tapered fibers. Therefore, different numbers of pulses with distinct amplitudes are observed in the output optical autocorrelation when considering different elongation points for the tunable tapered fiber. In addition, the effect of uncompensated chromatic dispersion in the experimental setup 
(a)

Electric Ground

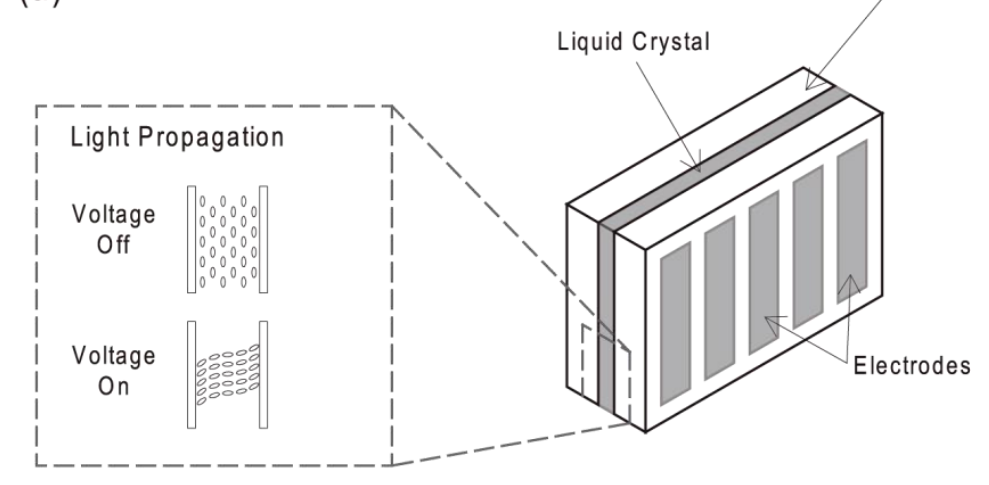

(b)
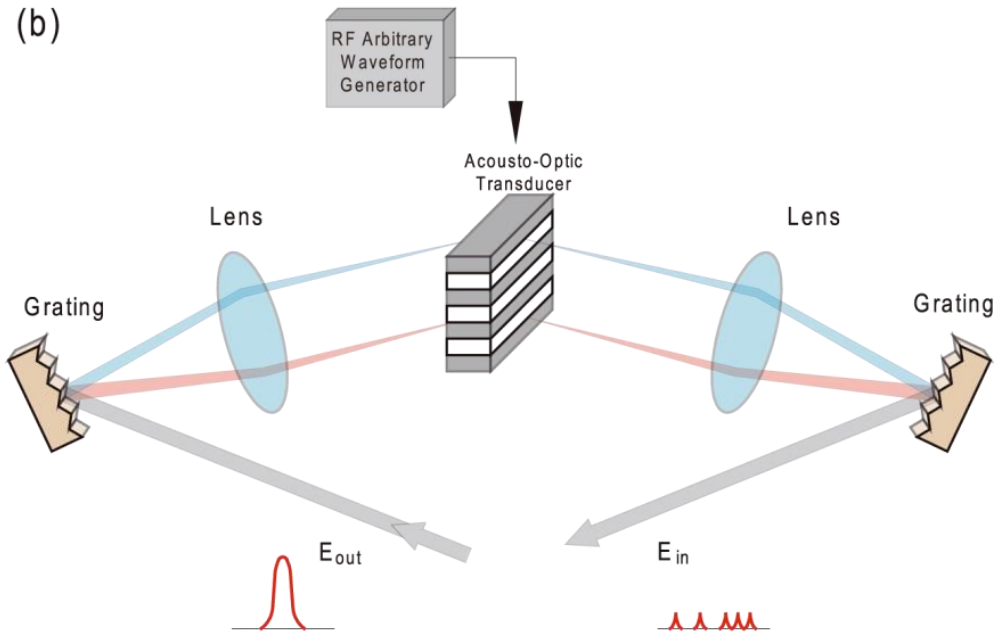

(c)

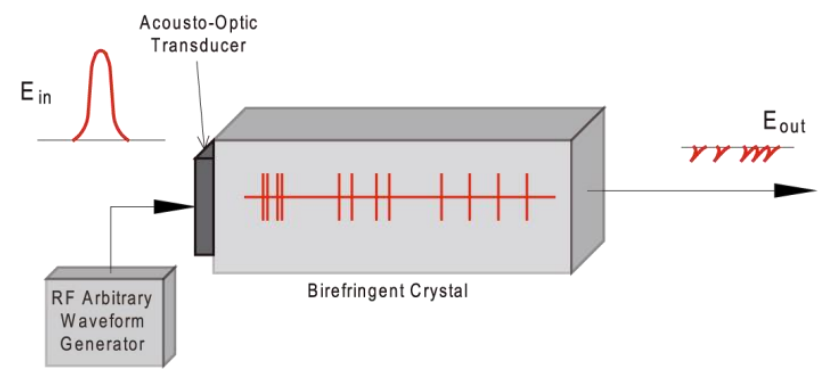

Figure 3.9: (a) Liquid crystal spatial light modulator (LC-SLM), (b) acousto-optic modulator (AOM), and (c) acousto-optic programmable dispersive filters (AOPDF) pulse shaper configurations. 
is analyzed for both normal and anomalous values. Results show the generation of a train of pulses due to the pulses overlapping which depends on the quantity of uncompensated dispersion present in the system.

\subsubsection{Sensing}

An optical sensor is a device based on optical technology capable of detecting a variation in the monitored medium. This variation causes a change in the sensor response that can be quantified and processed later. Optical sensors have many advantages such as electrical passiveness, Electromagnetic Interference (EMI) immunity, resistance to hostile environments such as high temperatures or chemical reagents, integration capabilities and mass production, high degree of biocompatibility, high resolution and sensitivity and remote operation capability, among others. Optical sensors are of widespread use in different fields such as industry, environmental control, physics, chemistry, medicine and biology [77].

Optical sensors can be used to detect different measurands such as temperature, pressure, displacement, refractive index, strain, vibration, rotation, acceleration, humidity, velocity and many more. To do this, optical sensors are designed in two possible configurations depending on where the interaction between the propagating light inside the fiber and the medium to be sensed is performed. In the so-called intrinsic optical sensors, the internal structure of the fiber converts the detected changes in the monitored medium into intensity, phase, frequency or polarization variations of the light propagating inside the fiber. Conversely, in the extrinsic optical sensors the detection and signal variation take place outside the fiber. Then, the modified signal is recoupled again inside the fiber [78]. In addition, optical sensors can be classified into three types depending on the surface used to interact with the medium under test as point sensor, distributed sensor and quasidistributed sensor [79]. Optical sensors of point type perform sensing in a particular location, such as a fiber tip, as shown in Fig. (3.10.a). On the other hand, distributed sensors employ a larger detection surface, such as a long length of fiber, as depicted in Fig. (3.10.b). Finally, the third type of sensor is the quasi-distributed optical sensor, which consists of a combination of the above two types. In this case, some specific sections located along the optical fiber are used in the sensing process. 
(a)

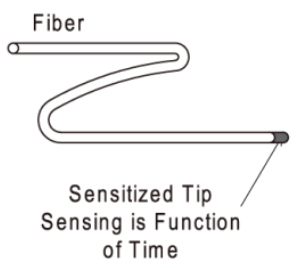

(b)

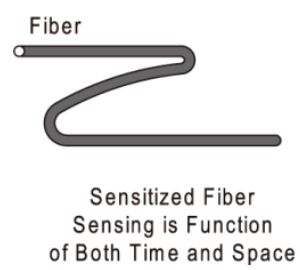

(c)

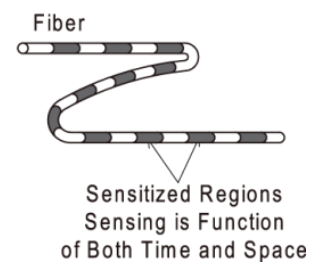

Figure 3.10: Classification of optical sensors depending on the sensing surface: (a) point sensor, (b) distributed sensor, and (c) quasi-distributed sensor.

An optical sensor is characterized by several parameters that quantify the sensor operation. The main parameters are the sensitivity, resolution, detection limit, response time, range operability and repeatability [80]. The sensitivity $S$ is defined as the spectral change experienced by the sensor response per unit of change. The resolution $R$ of a sensor is given by the smallest variation that can be accurately detected. From the relation between these two parameters, the detection limit $D L$ of the sensor, i.e. the minimum variation in the sensed medium that can be detected by the sensor, can be expressed as:

$$
D L=\frac{R}{S}
$$

Moreover, the response time $\tau$ can be defined as the time required for the sensor output to change from the initial state to $90 \%$ of the final value. The operating range is the interval between the maximum and the minimum value of the parameter to be sensed that can be measured. Finally, the repeatability is the parameter that indicates the variation produced between two equal measures at different time instants.

In recent years, many structures have been widely studied and demonstrated for different types of sensing measurements. The main developed devices are based on the use of optical fibers, photonic crystals, surface plasmon resonances, interferometric techniques and optical ring resonators [81], among others. Obviously, depending on the desired characteristics for the sensor, the technology to be employed must be carefully chosen. 
In optical fiber based sensors the main device used to carry out sensing measurements is a Fiber Bragg Grating, written inside the optical fiber. This periodic structure reflects a specific pulse component at wavelength $\lambda_{B}$, determined by $[79,82]$ :

$$
\lambda_{B}=2 n_{e f f} \Lambda
$$

where $\lambda_{B}$ is named Bragg wavelength, $n_{\text {eff }}$ is the effective refractive index of the core and $\Lambda$ is the grating pitch. Therefore, the operation principle of a FBG-based sensor lies in the detection of a spectral shift of the Bragg wavelength component as a function of the parameter to be measured, as seen in Fig. (3.11).

A modification realized on this structure that has attracted attention is the so-called Long Period Gratings (LPG), where the period is three or four orders of magnitude greater than in traditional FBGs. In LPG, the light propagating in the core mode is coupled into the cladding modes of the fiber for a particular wavelength given by [79]:

$$
\lambda_{L P G}=\left|n_{\text {core }}-n_{\text {cladding }}\right| \Lambda
$$

where $\mathrm{n}_{\text {core }}$ and $\mathrm{n}_{\text {cladding }}$ are the effective indexes of the core and the cladding, respectively. In this case, the sensitivity of the sensor is increased because the detection of the parameter under test is easier due to the increase in the field intensity. Moreover, Photonic Cristal Fibers (PCF) have also been used in the sensor fabrication because they exhibit advantageous characteristics such as high light confinement, which results in a high sensitivity value or the possibility to easily adjust the geometrical parameters of the structure in order to tailor the defect mode wavelength [83]. PCFs are periodic dielectric structures that give rise to a photonic bandgap. When a light beam impinges on the PCF, the spectral components whose wavelength is within the photonic bandgap are not propagated and therefore the PCF response presents a wide bandgap. However, if a defect is introduced into the core of the fiber, a transmission peak in the bandgap can be achieved, whose spectral position is very sensitive to changes in the external medium. Thus, sensing can be performed by the monitoring of the frequency shift experienced by the transmission peak. 

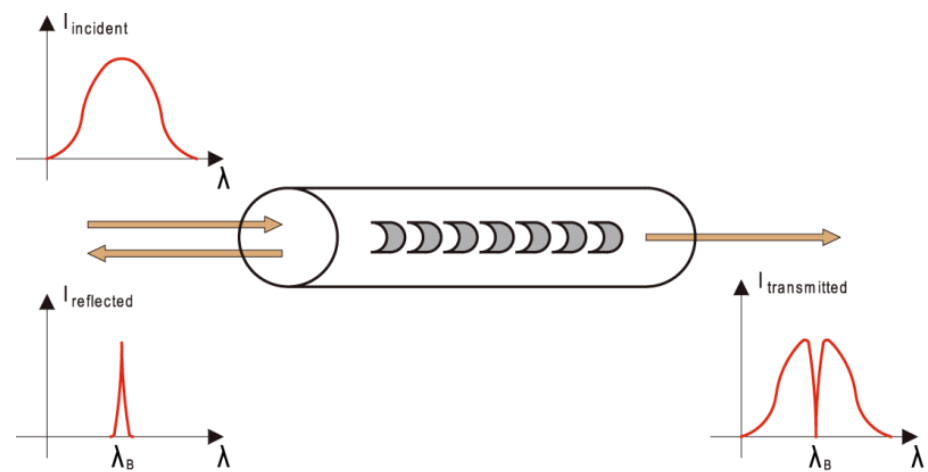

Applied

Perturbation
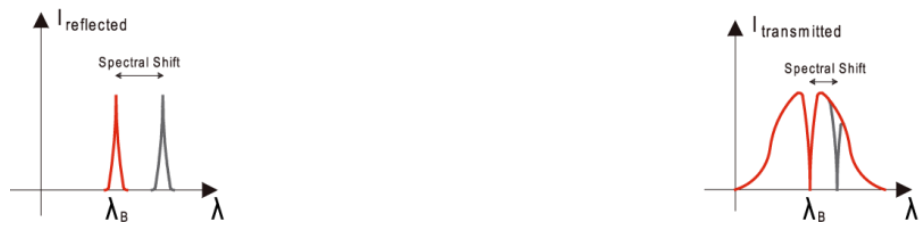

Figure 3.11: Operation principle of an optical fiber sensor based on Fiber Bragg Gratings.

Surface Plasmon Resonance (SPR) sensors are based on the detection of a change in the metal-dielectric interface of a structure by characterizing the spectral position of the resonances of the evanescent field propagating through the interface. These surface waves are called Surface Plasmon Waves (SPW) [81, 83]. There are four basic configurations in the SPR-based sensing: prism coupling, waveguide coupling, fiber coupling and grating coupling. These four methods are represented schematically in Fig. (3.12). In prism coupling, if the light propagating through the prism impinges at the metal-prism interface with a greater angle than the critical angle, it is totally reflected, thus generating an evanescent field that penetrates the metal layer. Waveguide coupling configuration presents a similar operation principle, wherein the light propagating through the waveguide generates an evanescent field at the waveguide-metal interface. This is also the case of fiber coupling, where the prism is replaced by a fiber where a small section is removed and then coated with a layer of metal. Lastly, grating coupling configuration occurs when light impinges in a metallic grating. 
(a)

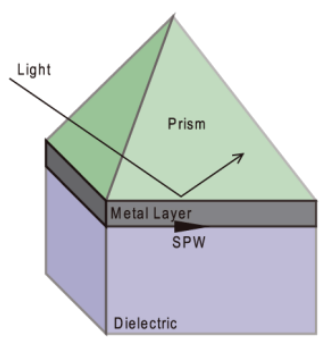

(c)

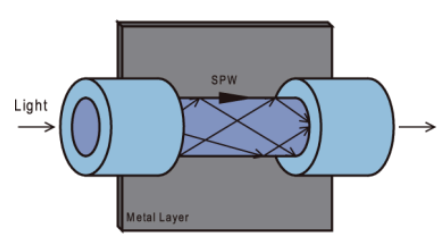

(b)

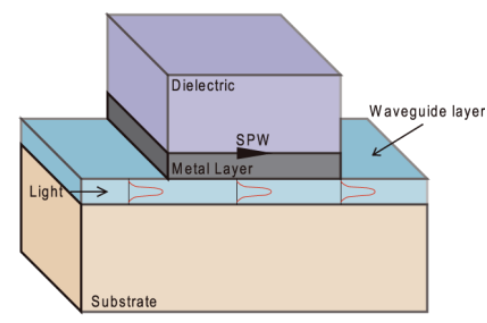

(d)

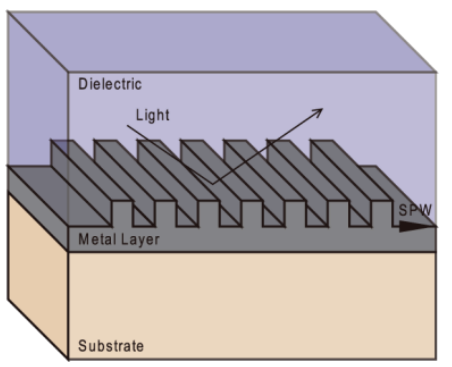

Figure 3.12: Configurations of surface plasmon resonance-based optical sensors (a) prism coupling, (b) waveguide coupling, (c) fiber coupling, and (d) grating coupling.

If the diffracted light parallel to the grating surface has the same propagation constant as the surface plasmon, light will be coupled to the SPW $[81,83]$.

Another technique employed in the realization of optical sensors is interferometry. Different types of interferometers have been used for sensing applications, such as Young interferometers or Hartman interferometers [81]. However, the most commonly used structure in interferometric based sensing is the Mach-Zehnder interferometer. In this type of device, a light beam is injected into the input waveguide and then is split at the Y-junction. One branch of the device is used as a reference, so that the light propagating along that path does not interact with the outer medium. However, the light propagating through the other arm of the interferometer interacts with the external medium through a window made in the simple cover, as shown in Fig. (3.13). 


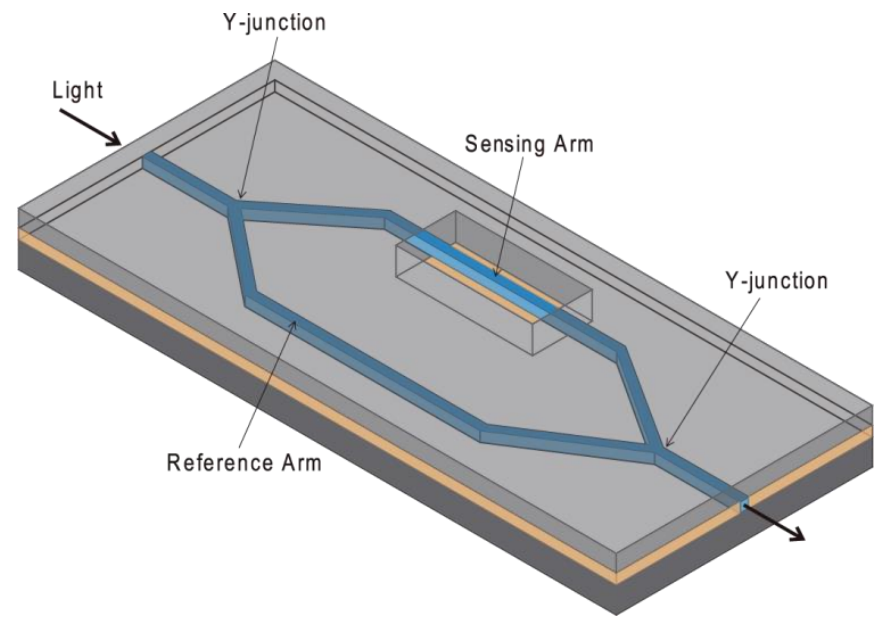

Figure 3.13: Mach-Zehnder interferometer-based sensor.

After that, the signals from both arms of the interferometer are recombined in the Y-junction located at the output waveguide generating an interference pattern whose changes depend on the optical phase variations detected in the sensing arm of the device.

The optical ring resonators based sensors make use of the Whispering Gallery Modes (WGM) or modes propagating inside the resonator, which have an evanescent field at the surface of the ring. When the evanescent field interacts with the outer medium, the response of the ring experiences a variation, as depicted in Fig. (3.14), because the resonance wavelength of the structure depends on the refractive index of the WGM as follows [81, 84]:

$$
\lambda_{\text {resonance }}=\frac{2 \pi r n_{\text {eff }}}{m}
$$

where $r$ is the radius of the ring, $n_{\text {eff }}$ is the effective index experienced by the resonant mode and $m$ is an integer. The effective interaction length of the ring with the surrounding medium is given by:

$$
L_{\text {eff }}=\frac{Q \lambda_{\text {resonance }}}{2 \pi n}
$$

where $Q$ is the quality factor and $n$ is the refractive index of the ring. The interaction length is related to the sensitivity of the ring sensor. Two 


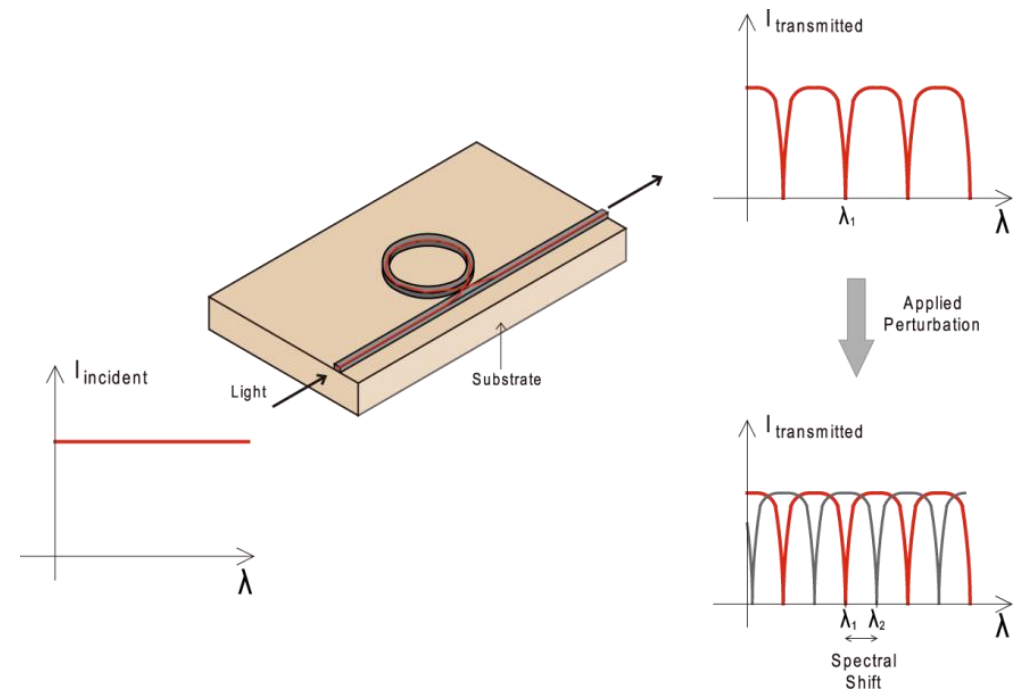

Figure 3.14: Optical ring resonator based optical sensor.

other main geometries of optical resonators based on the same operation principle have been widely implemented and studied: diskshaped resonators and microtoroid-shaped resonators [81, 84].

Low-cost refractive index and strain sensing by means of an in-line combination of two tapered fibers is presented in Paper E. Several surrounding mediums such as air, deionized water and $2 \%, 4 \%$ and $6 \%$ ethanol concentration in deionized water are considered in the refractive index sensing measurements. Strain measurements are realized by stretching one tapered fiber. The cost reduction in the setup is achieved by using a broadband source and a power meter instead of the tunable laser pumping at the maximum slope point used in traditional approaches. Although this affordable configuration can lead to lower sensitivity values, this disadvantage is overcome by using the cascaded combination. Both refractive index and strain sensing measurements using a tunable laser are also presented for comparison purposes. In addition, power stability in the tapered fiber response is analyzed when using the broadband source and the tunable laser, showing a higher stability in the case of the broadband source approach. 
Paper $\boldsymbol{F}$ provides a manipulation guideline when tapered fibers are used in refractive index and sensing measurements. Several aspects required in order to not alter the results in an inappropriate way or break down the tapered fiber are taken into account. The main consideration in refractive index measurements is related to the placement of the tapered fiber. As the evanescent field present in the fiber interacts with the surrounding medium and modifies the device response, an adequate location of the tapered fiber must be chosen to avoid undesired influences in the sensor response. Other issues such as the location of the fiber anchor points or the creation of bubbles in the container are also considered. Modifications in the tapered fiber transmission when an improper bending is introduced in the device are analyzed for strain sensing applications.

\subsubsection{Mode Conversion}

Mode conversion in an optical fiber consists of power coupling from one mode to another due to variations in the fiber geometry or imperfections in its refractive index distribution. Mode coupling is widely used in devices and applications such as directional couplers, wavelength multiplexors, fiber amplifiers, Mach-Zehnder interferometers or micro resonators, among others. This effect is usually analyzed by means of the Coupled-Mode Theory (CMT), a technique that splits light into a known number of modes propagating through the ideal structure and subsequently calculates the coupling relations between modes when the variation in the waveguide geometry or refractive index is introduced [85].

In this work, power conversion between modes is realized by considering the introduction of a curvature in the optical fiber. The main consequence of the presence of the bend in the light propagation through the optical fiber is the transformation from guided modes to radiated modes. In the resolution of the equations related to the energy distribution inside the bent fiber the weak guidance approximation is typically used, which approximates the weakly guided fiber modes by linearly polarized electric fields, leading to a simplification in the resolution of the equations due to a scalar solution [86]. These equations are expressed in a system of cylindrical polar local coordinates $r, \phi, z$, as 
shown in Fig. (3.15). Therefore, the transverse field component $E$ must be a solution of the wave equation given by:

$$
\frac{\partial^{2} E}{\partial r^{2}}+\frac{1}{r} \frac{\partial E}{\partial r}+\frac{1}{r^{2}} \frac{\partial^{2} E}{\partial \phi^{2}}+\frac{\partial^{2} E}{\partial z^{2}}+n^{2}[1+2(r / R) \cos \phi] k^{2} E=0
$$

where $n$ is the refractive index, $R$ is the radius of the curvature and $k$ is the free space propagation constant. In Eq. (3.19), the effect of the bend is contained in the term describing the effective refractive index:

$$
n_{e f f}=n[1+(r / R) \cos \phi]
$$

The solution of the wave equation describing the field distribution in a curved optical fiber is obtained by solving Eq. (3.19):

$$
E=e^{-i \beta_{g} z} \sum_{v \rho=1}^{\infty} A_{v \rho} J_{v}\left(\sigma_{v \rho} r\right) \cos v \phi
$$

where $\beta_{g}$ is the propagation constant of the guided mode, $A$ are the expansion coefficients of the method, $J$ represents the Bessel functions and $v$ and $\rho$ are the azimuthal and radial number mode, respectively [86, 87].

Once the modal field distribution is calculated, bend loss can be obtained as follows:

$$
2 \alpha=\frac{4\left|I_{1}\right|^{2}}{\pi^{3 / 2} \gamma^{1 / 2} R^{3 / 2} I_{2}\left|H_{\mu}^{(2)}(\xi)\right|^{2}}
$$

with

$$
\begin{aligned}
& I_{1}=\int_{0}^{r_{0}} E(z) d z \\
& I_{2}=\int_{0}^{2 \pi} d \phi \int_{0}^{r_{0}}|E|^{2} r d r \\
& \gamma=\sqrt{\beta_{g}^{2}-n_{2}^{2} k^{2}}
\end{aligned}
$$

where $\mu$ is the order number, $n_{2}$ is the refractive index of the material surrounding the core fiber and $H$ takes part in the expansion in term of Hankel functions. In the bending loss analysis in optical fibers, two different regions can be identified, the transition region and the pure bend region. 


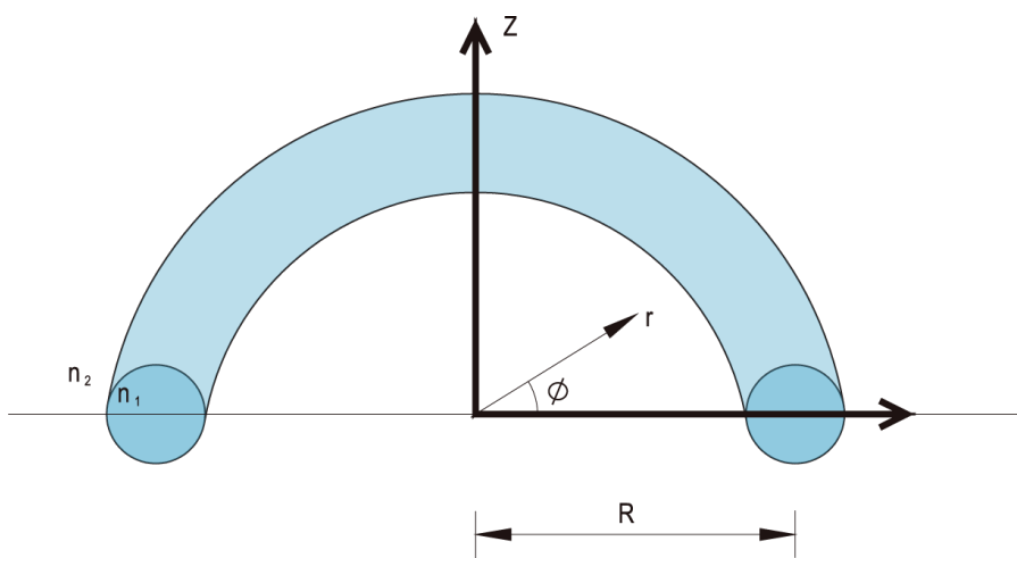

Figure 3.15: Local coordinates in a curved optical fiber.

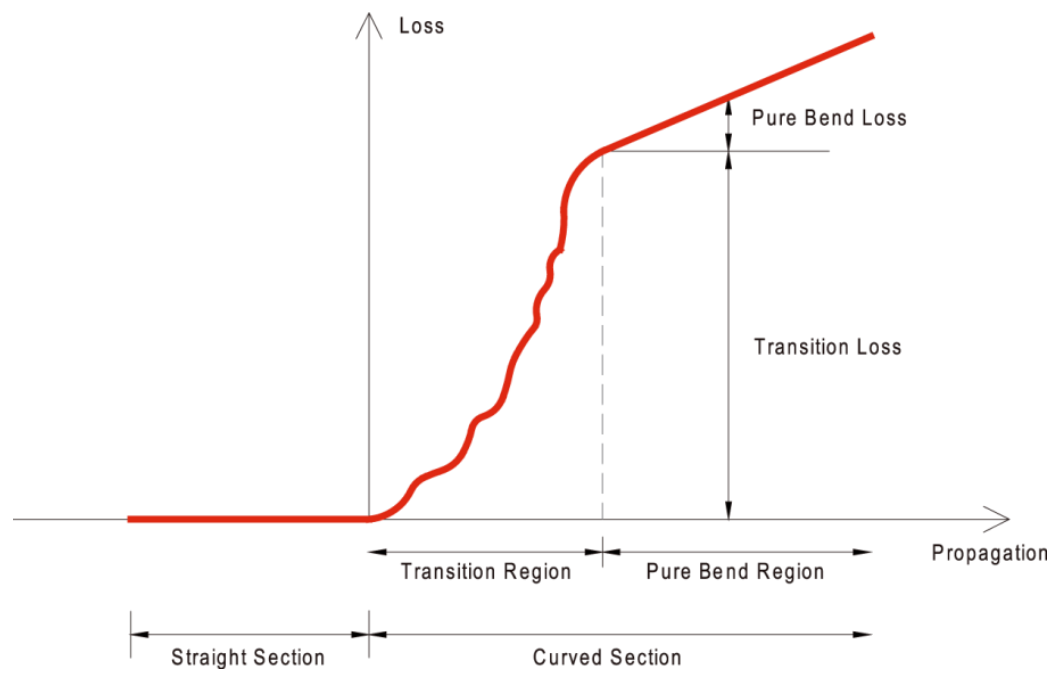

Figure 3.16: Transmission loss dependency on the curvature of the optical fiber.

In the transition region, losses are due to conversion from the mode propagating through the straight fiber to the curved mode. Moreover, in the pure bend region losses are due to the uniform curvature [88]. A scheme of the two different areas that contribute to the transmission 
loss distribution in a curved fiber can be shown in Fig. (3.16). When the phase velocity of the mode propagating guided within the fiber equals the speed of light, the guided mode becomes a radiated mode. Such radiation, which is emitted in the transverse direction of the curve, appears as discrete tangential rays varying as a function of the radius curvature [89]. As depicted in Fig. (3.16), the transition region presents oscillatory behavior, which is due to a forward and backward mode coupling between the guided mode and the leaky and cladding modes. Once the pure bend region is reached, beams progressively broaden and the radiated power becomes more uniform [90]. Also, note that the conversion between the transition region and the pure bend region depends on the radius of the curvature, resulting in shorter transition regions when considering smaller bend radii, since the leaky mode radiates faster. Furthermore, the energy distribution in a curved optical fiber differs from the typical energy distribution in a straight fiber as in the curved case the energy is shifted towards the outer boundary of the fiber $[89,91]$.

Mode conversion in a tapered fiber is presented in Paper G. Transformation between guided modes and radiating modes is forced by introducing a curvature in the fiber and the evolution of the energy distribution when considering different surrounding mediums such as air, deionized water and 5\% ethanol concentration in deionized water is analyzed. The splitting of modes in a straight tapered fiber is obtained and exposed for comparison purposes. In addition, refractive index and strain measurements are carried out by using the curved tapered fiber arrangement. RI sensing measurements are realized in the outer mediums mentioned above and strain sensing is carried out by stretching the tapered fiber. The proposed sensor is based on measuring the radiation loss provided by the curvature instead of conventional approaches which monitor the spectral shifts of the sensor response; this results in a simpler and cheaper sensor.

\subsubsection{Nonlinearities}

Nonlinear optics refers to the study of the interaction of intense laser light with matter, which leads to a variation in the optical properties of the material [92]. Nonlinear effects in optical fiber present both 
advantages and disadvantages. On the negative side, in Wavelength Division Multiplexing (WDM) communication systems, if the power threshold is exceeded, nonlinearities arise and can lead to the degradation of the signal [93]. However, the use of nonlinearities in many applications such as amplification, multiplexing and demultiplexing, wavelength conversion and regeneration, among others, has been extensively studied and characterized [94].

The core size and length of optical fiber make it ideal for applications governed by nonlinearities as they improve the nonlinearity in a factor given by $[94,95]$ :

$$
\frac{\left(I_{0} L_{e f f}\right)_{\text {fiber }}}{\left(I_{0} L_{\text {eff }}\right)_{\text {bulk }}}=\frac{\lambda}{\pi \omega_{0}^{2} \alpha}
$$

where $I_{0}$ is the intensity (power per area unit), $L_{e f f}$ is the effective length, $\lambda$ is the wavelength, $\omega_{0}$ is the radius of the fiber core and $\alpha$ is the fiber loss. Eq. (3.26) is fulfilled when the optical fiber is long enough to satisfy $\alpha L>>1$ where $L$ is the length of the fiber. As can be seen from Eq. (3.26), assuming the radius of the core to be small and low loss, nonlinearity efficiency can be improved considerably. Other parameters involved in dealing with nonlinearities in optical fiber are the core effective area, $A_{\text {eff }}$, the fiber effective length, $L_{\text {eff, }}$ and the group velocity dispersion, GVD [94]. The core effective area is the area that the core should have if the optical intensity were uniformly distributed within the nucleus and zero outside. The fiber effective length is the real length of the region where the optical intensity $I_{0}$ can be maintained. The effective length is loss and fiber length - dependent. In relation to the GVD, in order to operate at a low loss point and satisfy the phase matching condition, dispersion must be accurately designed and controlled.

The nonlinear optical response of a material to an applied optical field can be described by expressing the polarization as a series dependent on the electrical field [92]:

$$
\tilde{P}(t)=\chi^{(1)} \tilde{E}(t)+\chi^{(2)} \tilde{E}^{2}(t)+\chi^{(3)} \tilde{E}^{3}(t)+\cdots
$$

where $\chi^{(n)}$ is the $n$-th order susceptibility at optical frequencies. A medium is optically isotropic when the refractive index is constant for any light transmission direction through the medium. Because glass presents optical isotropy, the second-order susceptibility is usually zero. 
As a result, in an isotropic medium, the nonlinear response only depends on $\chi^{(3)}$. In addition, the nonlinear response of the material is assumed to depend instantaneously on the applied field.

There are many nonlinear optical processes associated with the third-order susceptibility. The real part of this susceptibility corresponds to the refractive index and the imaginary part corresponds to the phase delay in the response of the material. Depending on the origin of the nonlinearity, two groups of nonlinearities can be distinguished. On one hand, nonlinearities arising from the scattering and, on the other, nonlinearities arising from optically induced variations in the refractive index.

Scattering nonlinearities are those that occur due to vibrational dynamics in the glass. Therefore, in this type of nonlinearities, energy and momentum conservation laws of light and lattice must be satisfied [94]:

$$
\begin{aligned}
& \Omega=\omega_{L}-\omega_{S} \\
& \vec{q}=\vec{k}_{L}-\vec{k}_{S}
\end{aligned}
$$

where $L$ and $S$ refer to the laser and scattered signals respectively, $\omega$ and $k$ are the light frequency and wave vector and $\Omega$ and $q$ are the lattice phonon frequency and wave vector. The two scattering nonlinearities most common are Stimulated Brillouin Scattering (SBS) and Stimulated Raman Scattering (SRS).

\section{- Stimulated Brillouin Scattering:}

In spontaneous Brillouin process, a photon from the incident light wave becomes a photon and a scattered phonon [96]. The scattered wave, called Stokes wave, experiences a spectral shift to lower frequencies. The scattered light inside the fiber propagates both in forward and backward direction. However, Brillouin scattering in the forward direction is very weak and therefore the Stokes wave mainly propagates in the opposite direction in relation to the pump direction. When the pump power reaches a threshold value, the spontaneous process becomes a stimulated process, which is characterized by an energy conversion from the incident light to the scattered wave. In SBS, the backscattered Stokes wave interferes with the pump and generates 
an acoustic wave because of the electrostriction phenomenon that generates a density variation in the medium due to the interaction with light [97]. When the intensity of the Stokes wave rises, the interference pattern becomes more pronounced and the amplitude of the acoustic wave increases. The acoustic wave propagating in the forward direction acts as a Bragg grating and scatters light in the backward direction. A schematic of the Brillouin scattering process is shown in Fig (3.17). SBS is used in different applications such as remote sensing and ultranarrow linewidth lasers.

\section{o Stimulated Raman Scattering:}

In the Raman spontaneous effect, a light beam illuminates a material generating scattered light that presents different frequency components from those of the excitation source. The scattered components of lower frequency are denominated Stokes components, as in SBS effect, while the higher frequencies ones are called anti-Stokes components. Stokes components are typically several orders of magnitude higher than the anti-Stokes components. The Stimulated version of Raman scattering occurs when incident light comes from an intense light beam. In this case, approximately $10 \%$ of the energy from the incident wave is converted to Stokes frequencies [92]. Furthermore, stimulated Raman effect can be observed both in forward and backward directions although it is slightly higher in the forward direction. Moreover, both the generation power threshold and the frequency shift of the Stokes components are much larger than in SBS effect [94]. Fig. (3.18) shows a schematic describing Raman scattering. SRS phenomenon is used in many applications such as fiber amplifiers, lasers, optical modulation, switching and wavelength conversion, among others [94].

The other type of nonlinear effects considered in this work are the $\chi^{(3)}$ nonlinearities which arise from nonlinear changes in the refractive index due to the light incidence on the material. In this case, the refractive index can be expressed as [94]:

$$
n=n_{0}+n_{2} I
$$

where $n_{0}$ is the linear index, $n_{2}$ is the nonlinear index and $I$ is the optical intensity. The third-order susceptibility is related to the nonlinear coefficient by the following expression: 
(a)
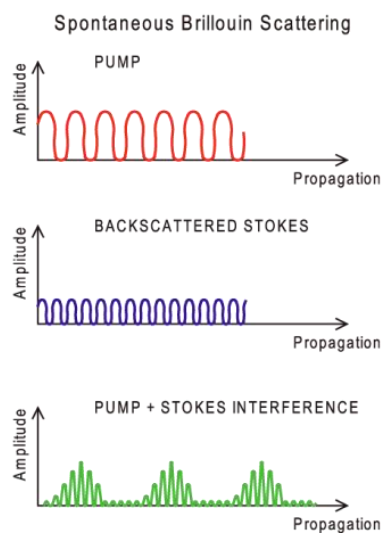

(b)
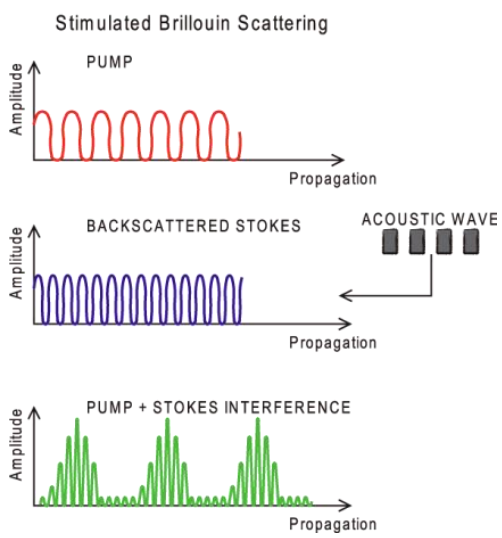

Figure 3.17: (a) Spontaneous and (b) Stimulated Brillouin Scattering.

(a)

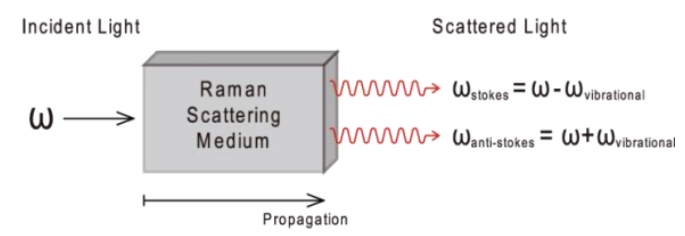

(b)

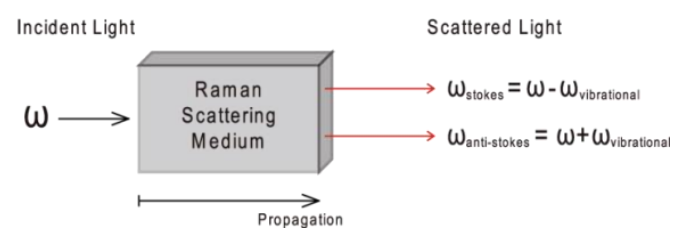

Figure 3.18: (a) Spontaneous and (b) Stimulated Raman Scattering.

$$
n_{2}=\frac{3}{8 n_{0}} \operatorname{Re}\left(\chi^{(3)}\right)
$$

The resulting effect of the $\chi^{(3)}$ nonlinearities is to introduce a nonlinear phase shift in the propagating light. The generated shift can be expressed as [94]:

$$
\phi_{N L}(z)=\gamma P_{0} z
$$


where $P_{0}$ is the peak input power and $\gamma$ is the nonlinear coefficient given by:

$$
\gamma=\frac{2 \pi}{\lambda} \frac{n_{2}}{A_{\text {eff }}}
$$

As the optical power depends on both space and time, $\chi^{(3)}$ nonlinearities can vary the mode-field distribution, as well as modify the pulse shape in the time domain and the spectral content in the frequency domain. Three different $\chi^{(3)}$ nonlinearities are explained in this work: Self-Phase Modulation (SPM), Cross-Phase Modulation (XPM) and FourWave Mixing (FWM).

\section{- Self-Phase Modulation:}

In the self-phase modulation phenomenon, intensity modulation of an optical beam results in the modulation of its own phase due to the modulation experienced by the refractive index of the medium [94]. The resulting time dependent change, or phase modulation, leads to a spectral broadening or a frequency chirping because the different pulse components experience different phase shifts, as can be seen in Fig. (3.19). The nonlinear spectral broadening can be either compensated or amplified by the chromatic dispersion of the fiber [98]. Thus, SPM depends on the temporal and spectral characteristics of the initial pulse, the chirp and the chromatic dispersion, among others. Self-phase modulation effect is widely used in temporal and spectral pulse compression, soliton generation and pulse regeneration [94].

\section{○ Cross-Phase Modulation:}

Cross-phase modulation effect is a similar phenomenon to SMP. However, in this case, the propagation of two optical beams overlapped in time and space is required [99]. Besides, when propagating optical pulses they must present similar GVD values for XPM to arise. The fundamentals of the cross-phase modulation effect are based on the intensity modulation of one beam, which results in a phase modulation in the other beam, leading to a frequency modulation that generates a spectrum broadening. As in SPM, fiber dispersion affects considerably the XPM effect. XPM can be used in many all-optical applications in communications networks such as wavelength conversion, demultiplexing or switching. However, this effect can cause serious 

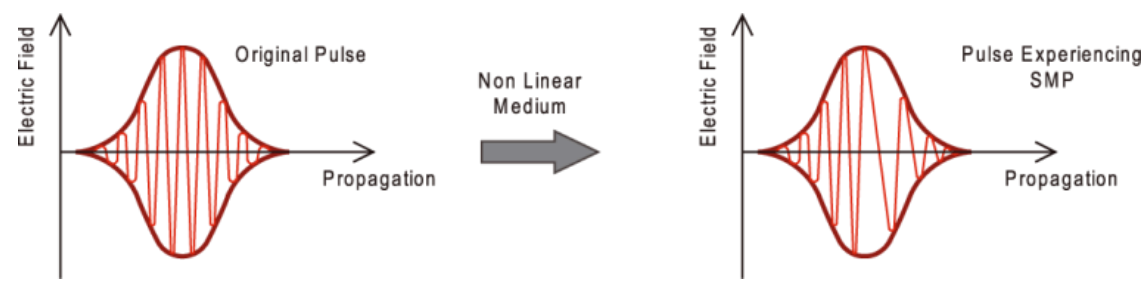

Figure 3.19: Self-Phase Modulation.

problems of crosstalk between channels in WDM communications systems [94].

\section{○ Four-Wave Mixing:}

In the four-wave mixing effect, three waves interact coherently between them to form a fourth one. Thus, the energy of two pumps at frequencies $\omega_{1}$ and $\omega_{2}$ is transferred to signal and idler waves. In addition to high enough power levels to generate the nonlinearity, FWM requires the phase-matching between the waves involved in the effect, i.e. their phase velocities are equal. Phase-matching condition must be verified over the sum of the wave vectors of the four waves that form the process as [13]:

$$
k_{1}+k_{2}=k_{3}+k_{4}
$$

Moreover, energy conservation must be satisfied:

$$
\omega_{1}+\omega_{2}=\omega_{3}+\omega_{4}
$$

When $\omega_{1} \neq \omega_{2}$ is met, it is called non-degenerated FWM. A special case of four wave mixing is the so called degenerated FWM, wherein $\omega_{1}=\omega_{2}$. Fig. (3.20) shows the non-degenerated and degenerated FWM schematic [100]. The main applications of the four-wave mixing effect are wavelength conversion, wavelength exchange, parametric amplification and optical regeneration, among others.

The Nonlinear Schrödinger Equation (NLSE) describes the propagation of optical ultrashort pulses in single-mode fibers. The generalized formulation of the expression is given by [13]:

$$
\frac{\partial A}{\partial z}+\frac{\alpha}{2} A+\frac{i \beta_{2}}{2} \frac{\partial^{2} A}{\partial T^{2}}-\frac{\beta_{3}}{6} \frac{\partial^{3} A}{\partial T^{3}}=i \gamma\left(|A|^{2} A+\frac{i}{\omega_{0}} \frac{\partial}{\partial T}\left(|A|^{2} A\right)-T_{R} A \frac{\partial|A|^{2}}{\partial T}\right)
$$


(a)

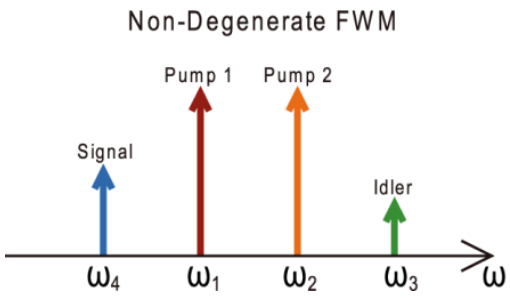

(b)

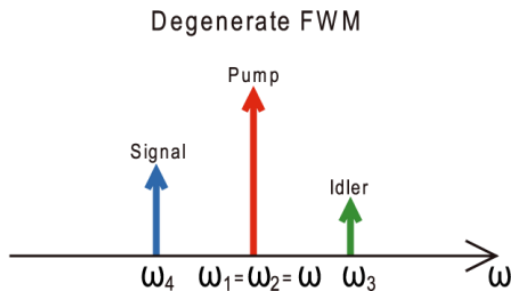

Figure 3.20: Four-Wave Mixing effect.

where $A$ is the complex field envelope, $\alpha$ is the fiber loss, $\beta_{2}$ and $\beta_{3}$ the second-order and third-order dispersion terms, $\gamma$ is the nonlinear coefficient and $\omega_{0}$ is the spectrum central frequency. Moreover, $T$ and $T_{R}$ can be expressed as follows [13]:

$$
\begin{aligned}
& T=\tau-\frac{z}{v_{g}} \\
& T_{R}=\int_{-\infty}^{\infty} t R(t) d t
\end{aligned}
$$

with $\tau$ being the physical time, $v_{g}$ being the group velocity and $R(t)$ being the response function. Considering pulses of width higher than 5 ps and neglecting several terms that become quite small, the nonlinear Schrödinger equation can be expressed in its simplified formulation as:

$$
i \frac{\partial A}{\partial z}+\frac{i \alpha}{2} A-\frac{\beta_{2}}{2} \frac{\partial^{2} A}{\partial T^{2}}+\gamma|A|^{2} A=0
$$

In most cases, NLSE cannot be solved analytically because it is a fourdimensional second-order partial differential equation. Therefore, a numerical method must be employed in order to approximate the vector equation to a scalar form. The two main resolution techniques are the socalled Finite Difference Methods (FDM) and the Pseudo spectral Methods $[13,101]$. FDM is based on solving the wave equations of Maxwell in the time domain by the paraxial approximation [102], which considers that the light propagation direction slightly deviates from the propagation axis. On the other hand, in pseudo spectral methods the spatial derivatives are represented in the spectral domain by means of a set of basis functions. Due to this spectral treatment, the pseudo spectral methods require a lower computational cost, being thus faster resolution 
methods. The Split Step Fourier Method (SSFM) is one of the most important pseudo spectral techniques because of its easy implementation $[13,101,103]$. In this method, the terms corresponding to the dispersion operator and the nonlinearity operator are decoupled and treated separately in the NLSE, which can be expressed as:

$$
\frac{\partial A}{\partial z}=(\widehat{D}+\widehat{N}) A
$$

where dispersion and nonlinearity operators are respectively given by:

$$
\begin{gathered}
\widehat{D}=-\frac{i \beta_{2}}{2} \frac{\partial^{2}}{\partial T^{2}}+\frac{\beta_{3}}{6} \frac{\partial^{3}}{\partial T^{3}}-\frac{\alpha}{2} \\
\widehat{N}=i \gamma\left(|A|^{2}+\frac{i}{\omega_{0}} \frac{1}{A} \frac{\partial}{\partial T}\left(|A|^{2} A\right)-T_{R} \frac{\partial|A|^{2}}{\partial T}\right)
\end{gathered}
$$

The operating principle of SSFM is shown in Fig. (3.21). Propagation along the fiber is evaluated in steps of length $h$, where it is assumed that the dispersion and nonlinearity operators act independently. First, nonlinearity is calculated in the midpoint of the step assuming $\widehat{D}=0$. Subsequently, dispersion is obtained considering that the nonlinearity is zero, $\widehat{N}=0$. This process is repeated for all the steps $[13,101]$.

Nonlinearity generation in non-adiabatic biconical tapered fibers is analyzed in Paper $\boldsymbol{H}$. The power threshold at which nonlinearities arise is determined by comparing the response of the tapered fiber when an initial chirped pulse distribution and pulses up to $10 \mathrm{~kW}$ are launched in the device. The nonlinearity generation effects in both temporal and spectral domains show an asymmetric spectral widening and a variable fringe visibility, which correspond to typical features of Self-Phase Modulation nonlinearity. Theoretical simulations are carried out solving the nonlinear Schrödinger equation by the Split-Step Fourier Method, showing a good agreement with the experiments. 

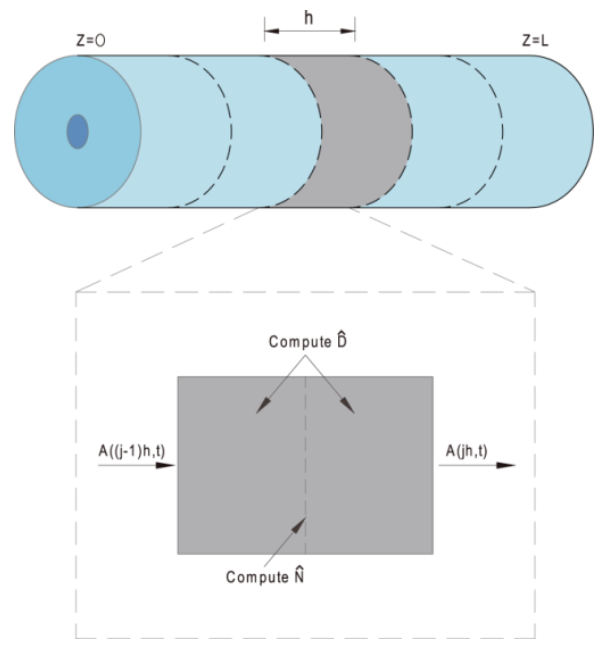

Figure 3.21: Split Step Fourier Method fundamentals (SSFM). 


\section{Chapter 4}

\section{General Discussion}

The objective of the previous chapters was to explain some important concepts required to understand the ideas developed and collected in the papers presented in this thesis and to provide the necessary context to frame this work. This chapter delivers a general discussion of the obtained results.

Chapter 1 introduces the basics of optical communications systems and focuses on chromatic dispersion, describing causes and consequences of this phenomenon. The broadening experienced by pulses propagating through the transmission medium is analyzed and quantified. Likewise, the two main contributions to chromatic dispersion, i.e. material and waveguide dispersion, are described. Having provided the theoretical basis of chromatic dispersion, its main experimental measurement schemes are presented. Techniques such as time-of-flight method, modulation-phase-shift method and interferometry method are explained. Furthermore, chromatic dispersion management motivation and necessity is exposed. The limitations induced by the dispersion in the system performance are analyzed and the signal degradation due to inter-symbol interference is highlighted. Dispersion compensation concept is also introduced, as well as the use of some types of fibers such as dispersion compensating fibers 
or dispersion shifted fibers for this purpose. The most common dispersion compensation arrangements are described, including the necessity of optical amplifiers and dispersion slope compensation. In addition, methodologies used in higher bit rate systems are considered.

Chapter 2 addresses the principles of chromatic dispersion tailoring in photonic integrated waveguides. Paper $\boldsymbol{A}$ presented here is framed in this context, where chromatic dispersion profiles of integrated slot waveguides are investigated. The effective refractive index of the modes propagating through the structure is computed through numerical simulations using commercial software and a subsequent differentiation process to obtain the chromatic dispersion profile. The aim of this paper is to analyze the influence of the waveguide's geometrical parameters on the dispersion curve in order to tune it. The results obtained show that two different dispersion regimes can be distinguished depending on the cross-sectional area of the waveguide. Some applications such as pulse shaping or nonlinearities, among others, are expected to benefit from this study.

Experimental measurements of dispersion in integrated optics are usually carried out by means of interferometry, mainly based on MachZehnder interferometers. The relation between the phase of the signal and the maximum and minimum positions in the spectral interference pattern obtained is used to do this. Paper $\boldsymbol{B}$ included in this chapter employs an experimental setup to measure chromatic dispersion based on a Mach-Zehnder interferometer. It allows for the calculation of continuous dispersion profiles as a function of wavelength while being immune to thermal fluctuations thanks to the counter propagating reference beam. When a wavelength sweep is realized, a continuous phase profile which subsequently allows the calculation of the experimental dispersion profile as a function of wavelength is obtained. This technique is used to measure dispersion of two types of geometries, strip and slot waveguides, as well as the group index of the structure. The results obtained show good agreement with theoretical calculations. The good performance of this technique is demonstrated even for devices with high insertion losses and polarization dependence.

Chapter 3 focuses on dispersion tailoring in tapered fibers. By heating and stretching a single-mode fiber, a different geometry presenting two transition regions and a narrow waist between them can 
be obtained. Aspects such as the tapered fiber geometry and its fabrication procedure are first examined. Then, the propagation characteristics are exposed, providing a description of the dependence of the tapered fiber response with the waist length and the difference between the effective indexes of the modes travelling along the waist. As explained, this allows carrying out dispersion engineering in this type of fibers by different procedures. Paper $\boldsymbol{C}$ presents experimental group delay and dispersion tailoring in tapered fibers by the application of strain in the taper. When mechanically stretching the fiber, its waist length as well as the effective indexes difference are modified, which results in a variation of the response of the tapered fiber. First, the group delay and the dispersion profiles of a single-mode fiber are obtained and compared with those corresponding to a fiber of the same characteristics but including a taper. Then, the tapered fiber is stretched to different points of elongations which allows for the tailoring of its dispersion profile. Group delay and dispersion engineering is also shown by means of the modification of the outer medium putting the tapered fiber in contact with a surface, leading to the possibility of coarse tailoring.

Some interesting applications in which tapered fibers are widely used such as supercontinuum generation and light coupling to micro resonators are also discussed. Furthermore, other functionalities carried out by means of tapered fibers are deeply described and demonstrated.

The first application considered, pulse shaping, relies on the modification of different characteristics of an optical pulse, such as envelope, amplitude or phase, to best suit the requirements of a specific application. The operating principle of pulse shaping is described and some of the most commonly arrangements used, i.e. 4f Fourier transform, liquid crystal spatial light modulator, acousto-optic modulator and acousto-optic programmable dispersive filters, presented. Paper $\boldsymbol{D}$ focuses on the use of an in-line combination of two tapered fibers to achieve dynamic pulse shaping in an affordable and simple way. To do this, one of the tapered fibers is mechanically stretched as to phase shift the envelope of the combined transmission response. This results in different pulse waveforms when stretching the tapered fiber to different elongations. This phase variation in the combined response arises from changes in the interference conditions between the transference functions of the individual tapered fibers. Additional flexibility is demonstrated by introducing different amounts 
of uncompensated dispersion in the experimental setup. Some applications that may benefit from our approach include terahertz generation and dynamic nonlinear optics.

Using one of the applications presented in Paper $\boldsymbol{D}$ as a guideline, the following functionality based on tapered fibers that was investigated was sensing. First of all, some aspects related to optical sensing are presented such as advantages, types of sensing and most common configurations. The performance parameters that characterize the sensor, such as sensitivity, resolution or detection limit, among others, are defined. Subsequently, the most popular structures and devices to perform sensing measurements are analyzed. Technologies considered suitable to build the sensor include optical fiber, photonic crystals, plasmonics and integrated waveguides. The next two papers presented in this thesis are related to sensing applications.

Paper $\boldsymbol{E}$ proposes a low cost experimental setup for refractive index and strain sensing measurements. The cost reduction arises from the use of an incoherent broadband light source instead of a tunable laser. Although this configuration leads to lower sensitivity values they can be increased by using an in-line combination of two tapered fibers. The refractive index measurements are carried out considering different outer mediums such as air, deionized water and different concentrations of ethanol in deionized water. Likewise, strain sensing measurements are realized by mechanically stretching one of the tapered fibers. A sensor arrangement comprising a tunable laser is also characterized for comparison purposes. Furthermore, sensitivity values obtained in both configurations for refractive index and strain sensing measurements are presented and compared.

As mentioned before, Paper $\boldsymbol{F}$ presented in this work is also framed in sensing functionalities. Its aim is to provide a set of rules for the manipulation of tapered fibers in refractive index and strain sensing measurements. The precautions necessary to handle such type of fibers in order to not introduce artifacts on the sensor's response are detailed and discussed. The main factors considered include the placement of the tapered fiber inside of the material which refractive index is to be sensed as well as the presence of undesired curvatures in strain sensing. Their effect on the sensor response is studied and compared with the proper handling case. 
Mode conversion is other functionality discussed in this work. It consists on the coupling of optical power from one propagating mode to another due to variations in the refractive index distribution or optical fiber geometry. Paper $\boldsymbol{G}$ presented in this work proposes a mode converter consisting of a mechanically stretched tapered fiber. A curvature is inserted in one of its transition regions, which allows the modification of the propagation characteristics of the device. By varying the radius the spectral response can be optimized. The proposed arrangement is used for refractive index and strain sensing, providing higher sensitivity when compared with the straight configuration sensor. Results show higher radiated power values for smaller bending radii, in good agreement with the theoretical fundamentals presented in this section. As demonstrated, the increased radiated power in the tapered fiber facilitates the detection mechanism and significantly improves the sensor performance. Furthermore, the applicability of the proposed structure to mode conversion is analyzed, showing the possibility of very high mode conversion ratios in a very simple way.

The last functionality reported is the generation of nonlinearities. First, the parameters and conditions required for nonlinear generation are presented. The nonlinear Schrödinger equation for the particular case of optical pulses propagating through optical fiber is described and two types of nonlinearities, the scattering nonlinearities and the $\chi^{(3)}$ nonlinearities, are differentiated. In the first case, stimulated Brillouin scattering and stimulated Raman scattering are considered. Then, $\chi^{(3)}$ nonlinearities such as cross-phase modulation, four-wave mixing and self-phase modulation are discussed. The generation of the latter effect in tapered fibers is the aim of Paper $\boldsymbol{H}$ presented in this thesis. In the first place, the power threshold required for nonlinear generation in the tapered fiber is determined. Subsequently, the response of the tapered fiber is obtained for different input power values in both temporal and spectral domains. Results show spectral broadening and variable fringe visibility, which are effects typically associated with self-phase modulation. The numerical results obtained after solving the nonlinear Schrödinger equation show good agreement with the experiments. 


\section{Chapter 5}

\section{Conclusions}

The great expansion experienced by optical communication systems has been facilitated by the multitude of advantages that presents its main transmission mediums, optical fiber and integrated waveguides. Among them, their high versatility has made it possible to develop multiple functionalities that cover a wide range of potential applications. These arise from the possibility of modifying the conventional characteristics of light propagation along the medium in a very easy way.

There are several undesired phenomena that must be taken into account in optical systems, such as losses, crosstalk and chromatic dispersion. Nevertheless, the possibility to control and tailor the chromatic dispersion present in an optical structure offers a wide range of applications. By a careful control of this phenomenon, several functionalities such as nonlinearities or ultrashort pulse propagation, among others, can be enhanced or suppressed. In this context, chromatic dispersion tailoring becomes a very powerful tool when designing optical communications systems. 
This PhD analyzes and implements chromatic dispersion tailoring in integrated photonics and optical fibers. To do this, both numerical and experimental studies are carried out. Several useful conclusions can be extracted from the obtained results.

First, different dispersion regimes are identified by means of software simulations by varying the geometrical parameters of integrated waveguides. This analysis can be used as a bidirectional design guideline: on one hand, it provides an approximated idea of the waveguide dimensions required to obtain a given dispersion profile; on the other hand, the quantity of dispersion for a given waveguide crosssection can be approximated.

Second, an experimental setup capable of measuring dispersion in integrated waveguides is developed. This arrangement provides continuous dispersion versus wavelength measurements without being affected by thermal fluctuations. Several functionalities can benefit from this, as it can be used directly for dispersion studies or as a starting point to develop other applications once the dispersion value of the integrated structure is known.

Third, dispersion tailoring in tapered fiber is analyzed and experimentally demonstrated. Tapered fibers have emerged as a very promising device because of its simplicity and wide applicability. In this $\mathrm{PhD}$, dispersion tailoring is easily carried out by applying stress to the fiber. The potential of this technique is the simplicity and the possibility of fine tuning the dispersion characteristics of the tapered fiber.

Finally, the use of tapered fibers in several functionalities such as pulse shaping, sensing, mode conversion and nonlinearities is also exposed and analyzed, in order to provide an idea of the versatility of this type of structure.

To sum up, such an important effect as chromatic dispersion is theoretically analyzed and experimentally measured in this PhD. Dispersion tailoring is carried out in both optical fiber and nanophotonic waveguides by modifying the geometry of the two transmission mediums. In addition, the applicability of tapered fibers for several functionalities is shown. 


\section{References}

[1] G.P. Agrawal, 'Fiber-Optic Communication Systems', WileyInterscience, Third Edition, 2002.

[2] H. Yin, and D.J. Richardson, 'Optical Code Division Multiple Access Communication Networks. Theory and Applications', Springer, First Edition, 2007.

[3] K.C. Kao, and G.A. Hockham, 'Dielectric-fibre surface waveguides for optical frequencies', IEE Proceedings 113, 7, 1151-1158, 1966.

[4] A. Werts, 'Propagation de la lumiere coherente dans les fibres optiques', L’Onde Electrique 46, 967-998, 1966.

[5] www.corning.com.

[6] G. Lifante, 'Integrated Photonics Fundamentals', John Wiley \& Sons Ltd, First Edition, 2003.

[7] R.G. Driggers, 'Enciclopedia of Optical Engineering. Volume 1', CRC, First Edition, 2003.

[8] H.J.R. Dutton, 'Understanding Optical Communications', IBM Corporation, First Edition, 1998.

[9] ISO 20473:2007, 'Optics and photonics - Spectral bands'.

[10] G. Keiser, 'Optical Fiber Communications', Tata McGraw-Hill, Fourth Edition, 2008.

[11] G. Keiser, 'Optical Communications Essentials', McGraw-Hill, First Edition, 2003.

[12] G.T. Reed, and A.P. Knights, 'Silicon Photonics. An introduction', John Wiley \& Sons, First Edition, 2004.

[13] G.P. Agrawal, 'Nonlinear Fiber Optics', Academic Press, Third Edition, 2001. 
[14] B. Tatian, 'Fitting refractive-index data with the Sellmeier dispersion formula', Applied Optics 23, 24, 4477-4485, 1984.

[15] L. Thevenaz, J.P. Pellaux, N. Gisin, and J.P. von der Weid, 'Review of Chromatic Dispersion Measurements Techniques', EFOC LAN 89, Seventh European Fibre Optic Communications and Local Area Networks 173763, 1989.

[16] M.A. Galle, 'Single-arm 3-wave interferometer for measuring dispersion in short lengths of fiber', Master Thesis, University of Toronto, 2007.

[17] L.G. Cohen, and C. Lin, 'Pulse delay measurements in the zero material dispersion wavelength region for optical fibers', Applied Optics 16, 12, 3136-3139, 1977.

[18] L.G. Cohen, and C. Lin, 'A Universal Fiber-Optic (UFO) Measurements System Based on a Near-IR Fiber Raman Laser', IEEE Journal of Quantum Electronics 14, 11, 855-589, 1978.

[19] B. Costa, D. Mazzoni, M. Puleo, and E. Vezzoni, 'Phase Shift Technique for the Measurement of Chromatic Dispersion in Optical Fibers Using LED's', IEEE Transactions on Microwave Theory and Techniques 30, 10, 1497-1503, 1982.

[20] M. Fujise, M. Kuwazuru, M. Nunokawa, and Y. Iwamoto, 'Chromatic dispersion measurement over a $100 \mathrm{~km}$ dispersion-shifted single-mode fibre by a new phase-shift technique', Electronics Letters 22, 11, 570$572,1986$.

[21] L.G. Cohen, 'Comparison of Single-Mode Fiber Dispersion Measurement Techniques', Journal of Lightwave Technology 3, 5, 958966, 1985.

[22] L. Thevenaz, J.P. Pellaux, and J.P. von der Weid, 'All-Fiber Interferometer for Chromatic Dispersion Measurements', Journal of Lightwave Technology 6, 1, 1-7, 1988.

[23] P. Merritt, R.P. Tatam, and D.A. Jackson, 'Interferometric Chromatic Dispersion Measurements on Short Lengths of Monomode Optical Fiber', Journal of Ligthwave Technology 7, 4, 703-716, 1989. 
[24] E. Dulkeith, F. Xia, L. Schares, M.J. Green, and Y.A. Vlasov, 'Group index and group velocity dispersion in silicon-on-insulator photonic wires', Optics Express 14, 9, 3853-3863, 2006.

[25] Y.A. Vlasov, M. O'Boyle, H.F. Hamann, and S.J. McNab, 'Active control of slow light on a chip with photonic crystal waveguides', Nature 438, 6569, 2005.

[26] D. Marcuse, 'Pulse distortion in single-mode fibers', Applied Optics 19, 10, 1653-1660, 1980.

[27] J. Heebner, R. Grover, and T.A. Ibrahim, 'Optical Microresonators. Theory, Fabrication and Applications', Springer, First Edition, 2008.

[28] G. Chauvel, 'Dispersion in Optical Fibers', White Paper, Anritsu Corporation, 2008.

[29] B. Collings, F. Haismann, and G. Lietaert, 'Reference Guide to Fiber Optic Testing. Volume 2', JDS Uniphase Corporation, 2010.

[30] C. Lin, H. Kogelnik, and L.G. Cohen, 'Optical-pulse equalization of low-dispersion transmission in single-mode fibers in the 1.3-1.7 $\mu \mathrm{m}$ spectral region', Optics Letters 5, 11, 476-478, 1980.

[31] Cisco, 'Fiber Types in Gigabit Optical Communications', White Paper, Cisco Systems Incorporation, 2008.

[32] L. Grüner-Nielsen, S. Nissen Knudsen, B. Edvold, T. Veng, D. Magnussen, C. Christian Larsen, and H. Damsgaard, 'Dispersion Compensating Fibers', Optical Fiber Technology 6, 164-180, 2000.

[33] A.J. Lowery, 'Optical OFDM', Proceedings of CLEO/QELS, 1-2, 2008.

[34] Z. Pan, Y.W. Song, C. Yu, Y. Wang, Q. Yu, J. Popelek, H. Li, and A.E. Willner, 'Tunable Chromatic Dispersion Compensation in 40-Gb/s Systems Using Nonlinearly Chirped Fiber Bragg Gratings', Journal of Lightwave Technology 20, 12, 2239-2246, 2002.

[35] M. Shirasaki, 'Virtually imaged phased array', Fujitsu Scientific and Technical Journal 35, 113-125, 1999. 
[36] B. Golubovic, R.R. Austin, M.K. Steiner-Shepard, M.K. Reed, S.A. Diddams, D.J. Jones, and A.G. Van Engen, 'Double Gires-Tournois interferometer negative-dispersion mirrors for use in tunable modelocked lasers', Optics Letters 25, 4, 275-277, 2000.

[37] A.C. Turner, C. Manolatou, B.S. Schmidt, M. Lipson, M.A. Foster, J.E. Sharping, and A.L. Gaeta, 'Tailored anomalous group-velocity dispersion in silicon channel waveguides', Optics Express 14, 10, 4357-4362, 2006.

[38] R.M. Osgood, N.C. Panoiu, J.I. Dadap, X. Liu, X. Chen, I-W. Hsieh, E. Dulkeith, W.M.J. Green, and Y.A. Vlasov, 'Engineering nonlinearities in nanoscale optical systems: physics and applications in dispersionengineered silicon nanophotonic wires', Advances in Optics and Photonics 1, 162-235, 2009.

[39] L. Zhang, Y. Yue, Y. Xiao-Li, J. Wang, R.G. Beausoleil, and A.E. Willner, 'Flat and low dispersion in highly nonlinear slot waveguides', Optics Express 18, 12, 13187-13193, 2010.

[40] L. Yin, Q. Lin, and G.P. Agrawal, 'Dispersion tailoring and soliton propagation in silicon waveguides', Optics Letters 31, 9, 1295-1297, 2006.

[41] M.A. Foster, A.C. Turner, J.E. Sharping, B.S. Schmidt, M. Lipson, and A.L. Gaeta, 'Broadband optical parametric gain on a silicon photonic chip', Nature 441, 960-963, 2006.

[42] C. Gunn, 'CMOS Photonics - SOI Learns a New Trick', IEEE International SOI Conference, 7-13, 2005.

[43] M. Lipson, 'Guiding, Modulating, and Emitting Light on Silicon Challenges and Opportunities', Journal of Lightwave Technology 23, 12, 4222-4238, 2005.

[44] J. Cardenas, C.B. Poitras, J.T. Robinson, K. Preston, L. Chen, and M. Lipson, 'Low loss etchless silicon photonic waveguides', Optics Express 17, 6, 4752-4757, 2009.

[45] RSoft Photonics CAD Suite 9.0.14, BeamProp User Manual. 
[46] D.T.H. Tan, K. Ikeda, P.C. Sun, and Y. Fainman, 'Group velocity dispersion and self-phase modulation in silicon nitride waveguides', Applied Physics Letters 96, 061101, 2010.

[47] H.K. Tsang, C.S. Wong, T.K. Liang, I.E. Day, S.W. Roberts, A. Harpin, J. Drake, and M. Asghari, 'Optical dispersion, two-photon absorption and self-phase modulation in silicon waveguides at $1.5 \mu \mathrm{m}$ wavelength', Applied Physics Letters 80, 416, 2002.

[48] W. Ding, C. Benton, A.V. Gorbach, W.J. Wadsworth, J.C. Knight, D.V. Skryabin, M. Gnan, M. Sorrel, and R.M. De La Rue, 'Solitons and spectral broadening in long silicon-on-insulator photonic wires', Optics Express 16, 5, 3310-3319, 2008.

[49] S.W. Harun, K.S. Lim, C.K. Tio, K. Dimyati, and H. Ahmad, 'Theoretical analysis and fabrication of tapered fiber', Optik 124, 538-543, 2013.

[50] T.A. Birks, and Y.W. Li, 'The Shape of Fiber Tapers', Journal of Lightwave Technology 10, 4, 432-438, 1992.

[51] H. Latifi, M.I. Zibaii, S.M. Hosseini, and P. Jorge, 'Nonadiabatic Tapered Optical Fiber for Biosensor Applications', Photonic Sensors 2, 4, 340-356, 2012.

[52] M. Sumetsky, Y. Dulashko, and A. Hale, 'Fabrication and study of bent and coiled free silica nanowires: Self-coupling microloop optical interferometer', Optics Express 12, 15, 3521-3531, 2004.

[53] L. Shi, Z. Chen, H. Liu, Y. Chen, Z. Ye, W. Liao, and Y. Xia, 'Fabrication of submicron-diameter silica fibers using electric strip heater', Optics Express 14, 12, 5055-5060, 2006.

[54] E.J. Zhang, W.D. Sacher, and J.K.S. Poon, 'Hydrofluoric acid flow etching of low-loss subwavelength-diameter biconical fiber tapers', Optics Express 18, 21, 22593-22598, 2010.

[55] J. Villatoro, D. Monzón-Hernández, and E. Mejía, 'Fabrication and modeling of uniform-waist single-mode tapered optical fiber sensors', Applied Optics 42, 13, 2278-2283, 2003. 
[56] J.D. Love, W.M. Henry, W.J. Stewart, R.J. Black, S. Lacroix, and F. Gonthier, 'Tapered single-mode fibres and devices. Part I: Adiabaticity criteria', IEE Proceedings-J 138, 5, 343-354, 1991.

[57] M.I. Zibaii, H. Latifi, Z. Saeedian, and Z. Chenari, 'Nonadiabatic tapered optical fiber sensor for measurement of antimicrobial activity of silver nanoparticles against Escherichia coli', Journal of Photochemistry and Photobiology B: Biology 135, 55-64, 2014.

[58] F. Gonthier, J. Lapierre, C. Veilleux, S. Lacroix, and J. Bures, 'Investigation of power oscillations along tapered monomode fibers', Applied Optics 26, 3, 444-449, 1987.

[59] C.M.B. Cordeiro, W.J. Wadsworth, T.A. Birks, and P.St.J. Russell, 'Engineering the dispersion of tapered fibers for supercontinuum generation with a $1064 \mathrm{~nm}$ pump laser', Optics Letters 30, 15, 19801982, 2005.

[60] R. Zhang, J. Teipel, X. Zhang, D. Nau, and H. Giessen, 'Group velocity dispersion of tapered fibers immersed in different liquids', Optics Express 12, 8, 1700-1707, 2004.

[61] M. Rusu, R. Herda, S. Kivistö, and O.G. Okhotnikov, 'Fiber taper for dispersion management in a mode-locked ytterbium fiber laser', Optics Letters 31, 15, 2257-2259, 2006.

[62] Z. Wang, H. Sone, Y. Tsuji, and M. Imai, 'Spectral Phase and Intensity Evolutions of Supercontinuum Generation in a Biconical Tapered Fiber', Optical Review 14, 2, 81-85, 2007.

[63] R. Zhang, X. Zhang, D. Meiser, and H. Giessen, 'Mode and group velocity dispersion evolution in the tapered region of a single-mode tapered fiber', Optics Express 12, 24, 5840-5849, 2004.

[64] J.M. Dudley, G. Genty, and S. Coen, 'Supercontinuum generation in photonic crystal fiber', Reviews of Modern Physics 78, 1135-1184, 2006.

[65] F. Lu, and W.H. Knox, 'Generation of a broadband continuum with high spectral coherence in tapered single-mode optical fibers', Optics Express 12, 2, 347-353, 2004. 
[66] S.M. Spillane, T.J. Kippenberg, O.J. Painter, and K.J. Vahala, 'Ideality in a Fiber-Taper-Coupled Microresonator System for Application to Cavity Quantum Electrodynamics', Physical Review Letters 91, 4, 043902, 2003.

[67] J.C. Knight, G. Cheung, F. Jacques, and T.A. Birks, 'Phase-matched excitation of whispering-gallery-mode resonances by a fiber taper', Optics Letters 22, 15, 1129-1131, 1997.

[68] A.M. Weiner, 'Ultrafast optical pulse shaping: A tutorial review', Optics Communications 284, 3669-3692, 2011.

[69] M. Wollenhaupt, A. Assion, and T. Baument, 'Handbook of Lasers and Optics', Springer, Second Edition, 2012.

[70] C.C. Chang, H.P. Sardesai, A.M. Weiner, 'Dispersion-free fiber transmission for femtosecond pulses by use of a dispersioncompensating fiber and a programmable pulse shaper', Optics Letters 23 , 4, 283-285, 1998.

[71] M.A. Dugan, J.X. Tull, and W.S. Warren, 'High-resolution acoustooptic shaping of unamplified and amplified femtosecond laser pulses', Journal of the Optical Society of America B 14, 9, 2348-2358, 1997.

[72] P. Tournois, 'Acousto-optic programmable dispersive filter for adaptative compensation of group delay time dispersion in laser systems', Optics Communications 140, 245-249, 1997.

[73] A.M. Weiner, J.P. Heritage, and E.M. Kirschner, 'High-resolution femtosecond pulse shaping', Journal of the Optical Society of America B 5, 8, 1563-1572, 1988.

[74] A.M. Weiner, 'Femtosecond pulse shaping using spatial light modulators', Review of Scientific Instruments 71, 1929-1960, 2000.

[75] N. Belabas, J.P. Likforman, L. Canioni, B. Bousquet, and M. Joffre, 'Coherent broadnabd pulse shaping in the mid infrared', Optics Letters 26, 10, 743-745, 2001. 
[76] Y. Park, M. Kulishov, R. Slavík, and J. Azaña, 'Picosecond and subpicosecond flat-top pulse generation using uniform long-period fiber gratings', Optics Express 14, 26, 12670-12678, 2006.

[77] D. Ahuja, and D. Parande, 'Optical sensors and their applications', Journal of Scientific Research and Reviews 1, 5, 60-68, 2012.

[78] S. Ghetia, R. Gajjar, and P. Trivedi, 'Classification of Fiber Optical Sensors', International Journal of Electronics Communication and Computer Technology 3, 4, 442-445, 2013.

[79] A.D. Kersey, 'A Review of Recent Developments in Fiber Optic Sensor Technology', Optical Fiber Technology 2, 291-317, 1996.

[80] G. Brambilla, 'Optical fibre nanotaper sensors', Optical Fiber Technology 16, 331-342, 2010.

[81] X. Fan, I.M. White, S.I. Shopova, H. Zhu, J.D. Suter, and Y. Sun, 'Sensitive optical biosensors for unlabeled targets: A review', Analytica Chimica Acta 620, 8-26, 2008.

[82] B. Lee, 'Review of the present status of optical sensors', Optical Fiber Technology 9, 57-79, 2003.

[83] B. Lee, S. Roh, and J. Park, 'Current status of micro- and nanostructured optical fiber sensors', Optical Fiber Technology 15, 209-221, 2009.

[84] Y. Sun, and X. Fan, 'Optical ring resonators for biochemical and chemical sensing', Analytical and Bioanalytical Chemistry 399, 205-211, 2011.

[85] D. Marcuse, 'Coupled Mode theory of Round Optical Fibers', The Bell System Technical Journal 52, 6, 817-842, 1973.

[86] D. Marcuse, 'Field deformation and loss caused by curvature of optical fibers', Journal of the Optical Society of America 66, 4, 311-320, 1976. 
[87] D. Marcuse, 'Curvature loss formula for optical fibers', Journal of the Optical Society of America 66, 3, 216-220, 1976.

[88] W.A. Gambling, H. Matsumura, C.M. Ragdale, and R.A. Sammut, 'Measurement of radiation loss in curved single-mode fibres', Microwaves, Optics and Acoustics 2, 4, 134-140, 1978.

[89] W.A. Gambling, D.N. Payne, and H. Matsumura, 'Radiation from curved single-mode fibres', Electronics Letters 12, 21, 567-569, 1976.

[90] W.A. Gambling, H. Matsumura, and R.A. Sammut, 'Mode shift at bends in single-mode fibres', Electronic Letters 13, 23, 695-697, 1977.

[91] W.A. Gambling, and H. Matsumura, 'Propagation Characteristics of Curved Optical Fibers', The Transactions of the Iece of Japan 6, 3, 196201, 1978.

[92] R.W. Boyd, 'Nonlinear Optics', Academic Press, Third Edition, 2008.

[93] L.G.L. Wegener, M.L. Povinelli, A.G. Green, P.P. Mitra, J.B. Stark, and P.B. Littlewood, 'The effect of propagation nonlinearities on the information capacity of WDM optical fiber systems: cross-phase modulation and four-wave mixing', Physica D 189, 81-99, 2004.

[94] J. Toulouse, 'Optical Nonlinearities in Fibers: Review, Recent Examples, and systems Applications', Journal of Lightwave Technology 23, 11, 3625-3641, 2005.

[95] G.P. Agrawal, 'Nonlinear fiber optics: its history and recent progress', Journal of the Optical Society of America B 28, 12, A1-A10, 2011.

[96] A. Kobyakov, M. Sauer, and D. Chowdhury, 'Stimulated Brillouin scattering in optical fibers', Advances in Optics and Photonics 2, 1, 1-59, 2010.

[97] R.E. Newnham, V. Sundar, R. Yimnirum, J. Su, and Q.M. Zhang, 'Electrostriction: Nonlinear Electromechanical Coupling in Solid Dielectrics', Journal of Physical Chemistry B 101, 48, 10141-10150, 1997. 
[98] J.M. Dudley, A.C. Peacock, and G. Millot, 'The cancellation of nonlinear and dispersive phase components on the fundamental optical fiber soliton: a pedagogical note', Optics Communications 193, 253-259, 2001.

[99] M.N. Islam, L.F. Mollenauer, R.H. Stolen, J.R. Simpson, and H.T. Shang, 'Cross-phase modulation in optical fibers', Optics Letters 12, 8, 625-627, 1987.

[100] O. Aso, M. Tadakuma, and S. Namiki, 'Four-wave mixing in optical fibers and its applications', Furukawa Review 19, 63-68, 2000.

[101] B.R. Washburn, 'Dispersion and nonlinearities associated with supercontinuum generation in microstructure fibers', PhD Dissertation, Georgia Institute of Technology, 2002.

[102] T. Takenaka, M. Yokota, and O. Fukumitsu, 'Propagation of light beams beyond the paraxial approximation', Journal of the Optical Society of America A 2, 6, 826-829, 1985.

[103] O.V. Sinkin, R. Holzlöhner, J. Zweck, and C.R. Menyuk, 'Optimization of the Split-Step Fourier Method in Modeling Optical-Fiber Communications Systems', Journal of Lightwave Technology 21, 1, 61-68, 2003. 
91 
Papers A-H 


\section{Paper A}

\section{"Tailoring the Dispersion Behavior of Silicon Nanophotonic Slot Waveguides"}

S. Mas, J. Caraquitena, J.V. Galán, P. Sanchis, and J. Martí

Optics Express, Volume 18, Number 20, pp. 20839-20844, 2010. 


\title{
Tailoring the dispersion behavior of silicon nanophotonic slot waveguides
}

\author{
Sara Mas, José Caraquitena, José V. Galán, Pablo Sanchis, and Javier Martí \\ Valencia Nanophotonics Technology Center, Universidad Politécnica de \\ Valencia
}

\begin{abstract}
We investigate the chromatic dispersion properties of silicon channel slot waveguides in a broad spectral region centered at $\sim 1.5 \mu \mathrm{m}$. The variation of the dispersion profile as a function of the slot fill factor, i.e., the ratio between the slot and waveguide widths, is analyzed. Symmetric as well as asymmetric geometries are considered. In general, two different dispersion regimes are identified. Furthermore, our analysis shows that the zero and/or the peak dispersion wavelengths can be tailored by a careful control of the geometrical waveguide parameters including the cross-sectional area, the slot fill factor, and the slot asymmetry degree.
\end{abstract}

\section{Introduction}

In the last few years, silicon photonics has emerged as an attractive and promising technology in the field of integrated optoelectronics. The recent progress in nanofabrication techniques has enabled the development of basic photonic building blocks on a silicon-on-insulator platform including light sources, modulators, and photodetectors [1-3]. The silicon photonics approach provides some advantages, including lower-cost and higher-integration, compared with more traditional solutions based on other materials, e. g., III-V semiconductor compounds or $\mathrm{LiNbO}_{3}$.

In general, any silicon-based photonic component is affected by chromatic dispersion. Then, the design and optimization of silicon photonic devices requires a very precise knowledge of the dispersion properties. In this context, the chromatic dispersion of a simple silicon waveguide with $\sim 6 \mu^{2}$ cross-sectional area was first measured by Tsang et at [4]. In this dimension regime, the light confinement is weak 
and the dispersion profile is primarily determined by the intrinsic silicon dispersion. In contrast, when the cross-sectional area is reduced, the optical confinement is stronger and, then, the effective dispersion is the result of the interplay between the material and the waveguide or geometrical dispersion [5-7]. In fact, a careful control of the waveguide shape and size allows for the tailoring of the group velocity dispersion (GVD) so that normal, anomalous, or even zero GVD can be achieved in the spectral region centered at $\sim 1.5 \mu \mathrm{m}$ [5-7].

On the other hand, the so-called silicon nanophotonic slot waveguides have been proposed and fabricated for different applications [8-10]. In these waveguide structures, the optical field is strongly confined in a very thin region of low refractive index material, which is sandwiched between two silicon layers. As a result, a high optical intensity is produced in a small area so that the nonlinear optical performance is highly enhanced [10]. An early analysis of the dispersion properties of symmetric slot waveguides was reported by Zheng et al. [11]. In [11], numerical simulations demonstrate that the GVD of slot waveguides is, in general, significantly quite different compared with the dispersion of traditional channel waveguides. However, the analysis by Zheng et al. was limited to a small spectral range of only $0.15 \mu \mathrm{m}$ centered at $\sim 1.55 \mu \mathrm{m}$ and the dispersion control capabilities were unexplored. Simulated results of GVD in a horizontal slot waveguide filled with silicon nanocrystals have also been reported [12]. In addition, more complex slotted waveguides have been proposed for dispersion compensation purposes [13].

In this paper, we perform a detailed analysis of chromatic dispersion in silicon channel slot waveguides. First, a simple channel waveguide is assumed [7] and two different dispersion regimes are identified. Next, we analyze the influence of a slotted region on the GVD of the channel waveguide by considering different slot fill factors. Again, the same previous two different dispersion regimes are distinguished. In this case, the slot fill factor determines the dispersion regime in which the guiding structure operates. We also analyze the GVD in asymmetric slot-based structures, i.e., when the slot is placed in a region different than the geometrical center of the waveguide $[14,15]$.

\section{Dispersion in nanophotonic slot waveguides}

Let us first consider a conventional silicon channel waveguide consisting on a silicon channel embedded in a silica cladding, as shown in Fig. 1(a). Throughout this paper, three different cross-sectional areas, $A=h w$, will be considered, $1 \mu \mathrm{m}^{2}, 0.5 \mu \mathrm{m}^{2}$, and $0.1 \mu \mathrm{m}^{2}$, for both, conventional and slot waveguides, with $h$ and $w$ being the height and width of the waveguide, respectively, as shown in Fig. 1. These transversal areas are 
similar to those considered in other related works in silicon [7] so fabrication should not be a problem by using conventional nanofabrication techniques [6,7,9]. In addition, for simplicity, a fixed aspect ratio of 1-to-1.5 (height-to-width) will be assumed in all cases. However, it is important to mention that the GVD profile also depends on this parameter, as demonstrated in [7]. Our numerical simulations are performed by using a full-vector mode solver based on the beam propagation method [16]. In Fig. 1(a) a typical electric field profile of the fundamental quasi-TE mode in the $x$ axis is plotted. The field distribution is confined in the silicon core although evanescent tails are found in the silica region.

By using the mode solver, we compute the effective index, $\mathrm{n}_{\text {eff }}(\lambda)$, in a broad spectral range and by numerical differentiation the GVD parameter as a function of wavelength, $D_{\lambda}=-\left(\lambda / \mathrm{c}_{0}\right) \mathrm{d}^{2}$ neff $/ \mathrm{d} \lambda^{2}$, is obtained. It is worth mentioning that our analysis includes the contribution of material dispersion to the GVD, by considering the Sellmeier equations for both silicon and silica [17]. In Fig. 2, we show the resultant GVD profiles for the three cross-sectional areas under analysis. For comparison, the normal dispersion of pure crystalline silicon is also plotted. On the one hand, note that for larger cross-sectional areas, the GVD profile is similar to that corresponding to the silicon dispersion in such a way that the GVD gradually increases for longer wavelengths. We name this GVD behavior as material dispersion regime. A vertical up shifting in the dispersion profile is observed so that, eventually, we find a zero-GVD wavelength and, then, a region with anomalous GVD. The more the cross-sectional area is reduced, the more the zero-GVD wavelength is decreased. On the other hand, for smaller areas, the GVD profile is quite different having a maximum GVD along the spectral region and two zeroGVD wavelengths when the maximum dispersion value is positive, as shown in the figure. These GVD characteristics describe the geometrical dispersion regime. For other aspect ratios in this dispersion regime, similar dispersion profiles are obtained but we find normal GVD in the whole spectral range [7]. Two different qualitative dispersion of the
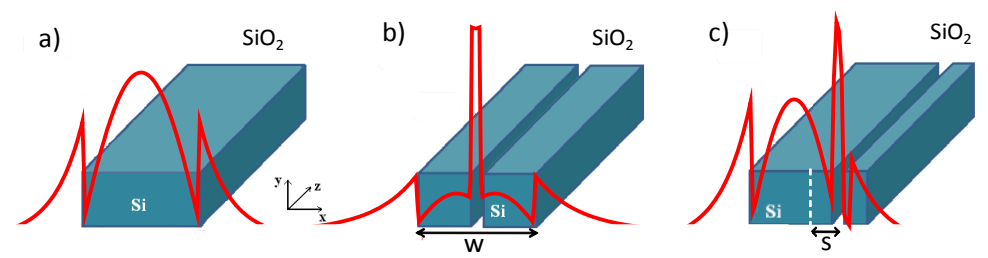

Fig. 1. (a) Conventional, (b) symmetric slot, and (c) asymmetric slot silicon-on-insulator channel waveguides with same cross sectional area. The electric field distribution of the quasi-TE mode in the $x$ dimension corresponding to $\lambda=1.55 \mu \mathrm{m}$ is plotted. 


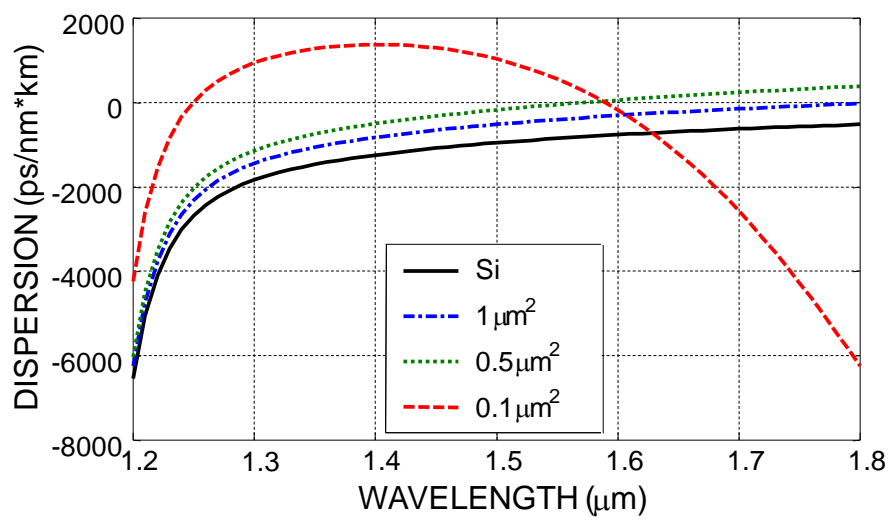

Fig. 2. Group velocity dispersion for the fundamental quasi-TE mode of a silicon nanophotonic channel waveguide with a fixed aspect ratio of 1:1.5 and three different cross sectional areas. The intrinsic pure crystalline silicon dispersion is also plotted.

characteristics are then observed depending on the cross-sectional area channel waveguide. Although beyond the scope of this paper, we mention that a more rigorous description of these two dispersion regimes can be performed by analyzing the sign of the next higher order dispersion parameter [5]. In the material dispersion regime, this term will be strictly positive in the whole spectral region but in the geometric dispersion regime we could find spectral regions with different sign and thus specific wavelengths where the term is cancelled.

\subsection{Symmetric slot waveguides}

We now turn our attention to the case of silicon waveguides with a vertical slot. In Fig. 1(b), a typical geometry of a slot waveguide is shown. Note that the modal electric-field distribution has a strong discontinuity at the high-index-contrast interfaces and the optical field is significantly increased in the slot region. We have computed the dispersion properties of three different slot waveguides with the above introduced cross-sectional areas, i.e., $1 \mu \mathrm{m}^{2}, 0.5 \mu \mathrm{m}^{2}$, and $0.1 \mu \mathrm{m}^{2}$, which include the slot region. The same aspect ratio is assumed. The resultant GVD curves are shown in Fig. 3(a-c), respectively. For each cross-sectional area, different slot fill factors have been considered, namely, 1:5, 1:10, 1:25, and 1:50. The fill factor is defined as the normalized ratio between the slot and the waveguide widths, i.e., $t / w$, see Fig. 1(b). Generally, in Figs. $3(\mathrm{a})-3(\mathrm{c})$ the dispersion profiles can be grouped into the two dispersion regimes previously defined for a conventional waveguide. As expected, 

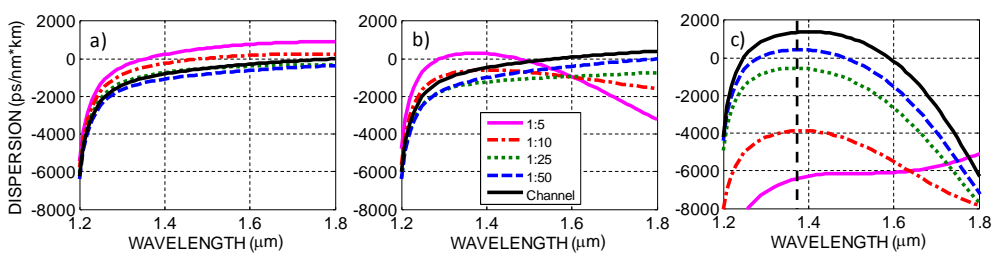

Fig. 3. Group velocity dispersion profiles of symmetric slot waveguides for different slot fill factors. Three different cross-sectional areas have been considered: (a) $1 \mu \mathrm{m}^{2}$, (b) $0.5 \mu \mathrm{m}^{2}$, and (c) $0.1 \mu \mathrm{m}^{2}$, with a fixed aspect ratio equal to $1: 1.5$. The dispersion curve of a conventional channel waveguide is also plotted.

when the slot fill factor is decreased, the GVD profiles asymptotically converge to the dispersion of conventional channel waveguides.

The effect of the slot on the waveguide dispersion is different for each particular cross- sectional area. For a cross-sectional area equal to 1 $\mu \mathrm{m}^{2}$, Fig. 3(a), a change in the slot fill factor translates into a relatively small variation in the GVD curve. In fact, all the dispersion profiles lie in the so-called material dispersion regime. Note that for larger fill factors, the dispersion profile exhibits a zero-GVD wavelength and, as a result, a spectral region with anomalous dispersion is found. For intermediate cross-sectional areas, $\sim 0.5 \mu \mathrm{m}^{2}$, Fig. $3(\mathrm{~b})$, we find that the slot dimension strongly determines the dispersion regime in which the waveguide operates. More particularly, for the fill factors 1:5 and 1:10 we have GVD profiles in the geometrical dispersion regime while the fill factors 1:25 and 1:50 present GVD curves quite similar to the silicon material dispersion profile. For small cross-sectional areas, $0.1 \mu \mathrm{m}^{2}$, Fig. 3(c), we find that the slot waveguide mostly works in the geometrical dispersion regime. Note that the dispersion curve is significantly down shifted when the slot fill factor is increased while the wavelength with maximum-GVD is nearly constant at $\sim 1.4 \mu \mathrm{m}$ (dashed vertical line). Transversal dimensions $h=258 \mathrm{~nm}$ and $w=387 \mathrm{~nm}$ are considered in this latter case, according to the assumed aspect ratio.

\subsection{Asymmetric slot waveguides}

In asymmetric silicon slot waveguides, the slot location is different than the geometrical center of the waveguide [14,15], as shown in Fig. 1(c). We define the asymmetry degree as $k=2 s / w$, where $s$ is the distance from the center of the waveguide to the center of the slot, in absolute value, and $w / 2$ is half of the total width of the waveguide, as shown in Fig. 1. With this definition, symmetric slot waveguides have an asymmetry degree equal to zero. We have analyzed the GVD for different asymmetry degrees, namely, $k=0,0.25,0.5$, and 0.75 while keeping the same cross-sectional area. Our aim is to investigate the influence of the 
waveguide asymmetry on the chromatic dispersion properties for the same cross-sectional areas assumed in previous simulations.

Figure 4, shows the results from numerical simulations for the three different above introduced cross-sectional areas (columns) and three different slot fill factors, 1:5, 1:10, and 1:25 (rows). Note that, according to our asymmetry degree definition, a specific slot shift to the right or to the left from the center yields the same asymmetry degree. As expected, the same GVD is obtained for both cases, as verified by numerical simulations. In general, we note from obtained results that the GVD is more sensitive to asymmetry changes when smaller cross-sectional areas are considered. For large areas, $1 \mu \mathrm{m}^{2}$, and for all the fill factors, Figs. 4(a)-4(c), the waveguide always operates in the material dispersion regime and a small change in the GVD is observed when the asymmetry degree is increased. For intermediate areas, $0.5 \mu \mathrm{m}^{2}$, more significant changes are found in the GVD. On the one hand, in Figs. 4(d-4e), by starting in the geometrical dispersion regime, a change in the asymmetry degree modifies the dispersion profile in such a way that the maximumdispersion wavelength shifts to longer values. For larger asymmetry degrees, $k=0.75$, the GVD is switched from the geometrical to the material dispersion regime. On the other hand, for a smaller fill factor, 1:25, Fig. 4(f), the GVD first changes from the material to the geometrical dispersion regime as the asymmetry degree is increased returning to the original behavior when the asymmetry is further increased. Finally, for small cross-sectional areas, $0.1 \mu \mathrm{m}^{2}$, Figs. $4(\mathrm{~g})-4(\mathrm{i})$, we find a significant larger GVD variation compared with previous examples. Note the different scales in the dispersion axis. For a fill factor equal to 1:5, Fig. $4(\mathrm{~g})$, when the asymmetry degree is increased the GVD enters into a new dispersion regime in which the dispersion profile exhibits both a maximum and a minimum dispersion value along the spectral region. This dispersive behavior is consistent with the GVD profiles obtained for conventional channel waveguides with cross-sectional areas smaller than $\sim 0.1 \mu \mathrm{m}^{2}$ [18]. For larger fill factors, Figs. 4(h),4(i) the waveguide exclusively operates in the geometrical dispersion regime for all the asymmetry degrees but exhibits a variation in the maximum-dispersion wavelength for a 1:10 fill factor, Fig. 4(h), whereas a nearly constant maximum-dispersion wavelength is observed for a 1:25 fill factor, Fig. 4(i). Interestingly, the cases $k=0.5$ in Fig. 4(e) and $k=0$ in Fig. 4(g) exhibit quite flat dispersion profiles over a certain spectral range. We attribute this behavior to a transition between different dispersion regimes. For all the cross-sectional areas, we have performed similar simulations by considering a fill factor equal to 1:50 obtaining small changes in the dispersion curves when the asymmetry in the waveguide is varied. 


\section{Conclusions}

A detailed analysis of the dispersion properties of silicon-on-insulator vertical slot waveguides has been performed. Our study shows that, in general, the dispersion behavior of slot waveguides strongly depends on the slot dimension and location. Two different dispersion regimes have been qualitative distinguished by analyzing and comparing several waveguide examples with different cross-sectional areas and slot fill factors. Our results show that a careful control of the slot geometrical parameters, i.e., width and position, enables the tuning of the GVD characteristics, including the maximum and/or the zero-GVD wavelengths. Furthermore, constant dispersion in a broad wavelength range can be achieved by properly designing slot waveguides. Dispersion tailoring of slot waveguides may be interesting for controlling several relevant phenomena including ultrashort pulse propagation and nonlinear optical effects.
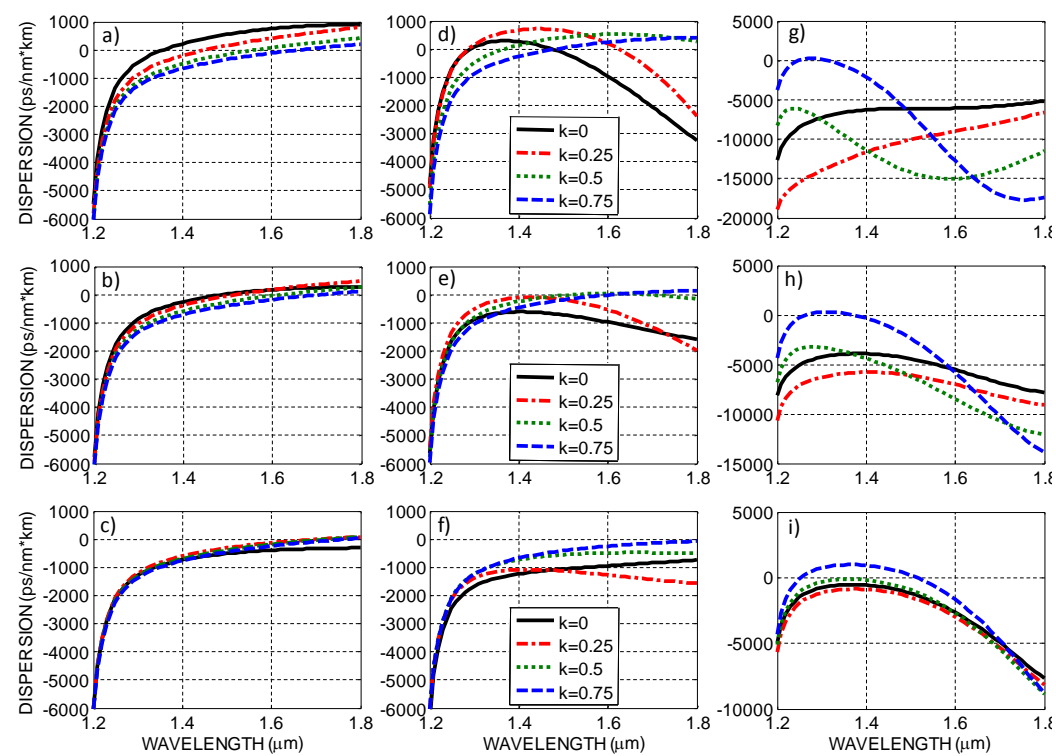

Fig. 4. Dispersion profiles of a slot waveguide for different asymmetry degrees. Three different cross-sectional areas are considered (a-c) $1 \mu \mathrm{m}^{2}$, (d-f) $0.5 \mu \mathrm{m}^{2}$, and (g-i) 0.1 $\mu \mathrm{m}^{2}$. Each row corresponds to a constant slot fill factor, 1:5, 1:10, and 1:25, respectively. The aspect ratio in all cases is 1:1.5. 


\section{References}

[1] M. Lipson, 'Guiding, modulating, and emitting light on silicon Challenges and opportunities,' Journal of Lightwave Technology 23, 12, 4222-4238, 2005.

[2] R. Soref, 'The past, present, and future of silicon photonics,' IEEE Journal of Selected Topics in Quantum Electronics 12, 6, 1678-1687, 2006.

[3] B. Jalali and S. Fathpour, 'Silicon photonics,' Journal of Lightwave Technology 24, 12, 4600-4615, 2006.

[4] H. K. Tsang, C. S. Wong, T. K. Liang, I. E. Day, S. W. Roberts, A. Harpin, J. Drake, and M. Asghari, 'Optical dispersion, two-photon absorption and self-phase modulation in silicon waveguides at $1.5 \mu \mathrm{m}$ wavelength,' Applied Physics Letters 80, 3, 416-418, 2002.

[5] L. Yin, Q. Lin, and G. P. Agrawal, 'Dispersion tailoring and soliton propagation in silicon waveguides,' Optics Letters 31, 9, 1295-1297, 2006.

[6] E. Dulkeith, F. Xia, L. Schares, M. J. Green, and Y. A. Vlasov, 'Group index and group velocity dispersion in silicon-on-insulator photonic wires,' Optics Express 14, 9, 3853-3863, 2006.

[7] A. C. Turner, C. Manolatou, B. S. Schmidt, M. Lipson, M. A. Foster, J. E. Sharping, and A. L. Gaeta, 'Tailored anomalous group-velocity dispersion in silicon channel waveguides,' Optics Express 14, 10, 4357-4362, 2006.

[8] V. R. Almeida, Q. Xu, C. A. Barrios, and M. Lipson, 'Guiding and confining light in void nanostructure,' Optics Letters 29, 11, 12091211, 2004.

[9] Q. Xu, V. R. Almeida, R. R. Panepucci, and M. Lipson, 'Experimental demonstration of guiding and confining light in nanometer-size low-refractive-index material,' Optics Letters 29, 14, 1626-1628, 2004.

[10] P. Sanchis, J. Blasco, A. Martínez, and J. Martí, 'Design of siliconbased slot waveguide configurations for optimum nonlinear performance,' Journal of Lightwave Technology 25, 5, 1298-1305, 2007.

[11] Z. Zheng, M. Iqbal, and J. Liu, 'Dispersion characteristics of SOI-based slot optical waveguides,' Optics Communications 281, 20, 5151$5155,2008$. 
[12] R. Spano, J. V. Galán, P. Sanchis, A. Martínez, J. Martí, and L. Pavesi, 'Group velocity dispersion in horizontal slot waveguides filled by Si nanocrystals,' International Conference on Group IV Photonics, 314316, 2008.

[13] L. Zhang, Y. Yue, Y. Y. Xiao-Li, R. G. Beausoleil, and A. E. Willner, 'Highly dispersive slot waveguides,' Optics Express 17, 9, 70957101, 2009.

[14] P. A. Anderson, B. S. Schmidt, and M. Lipson, 'High confinement in silicon slot waveguides with sharp bends,' Optics Express 14, 20, 9197-9202, 2006.

[15] C. Ma, Q. Zhang, and E. Van Keuren, 'Analysis of symmetric and asymmetric nanoscale slab slot waveguides,' Optics Communications 282, 2, 324-328, 2009.

[16] K. Okamoto, 'Fundamentals of Optical Waveguides', 1 1' Edition, Academic Press, 2000.

[17] B. Tatian, 'Fitting refractive-index data with the Sellmeier dispersion formula,' Applied Optics 23, 24, 4477- 4485, 1984.

[18] M. A. Foster, A. C. Turner, M. Lipson, and A. L. Gaeta, 'Nonlinear optics in photonic nanowires,' Optics Express 16, 2, 1300-1320, 2008. 


\title{
Paper B
}

\section{“Accurate Chromatic Dispersion Characterization of Photonic Integrated Circuits"}

\author{
S. Mas, J. Matres, J. Martí, and C.J. Oton \\ IEEE Photonics Journal, Volume 4, Number 3, pp. 825-831, \\ 2012.
}




\title{
Accurate chromatic dispersion characterization of photonic integrated circuits
}

\author{
S. Mas, J. Matres, J. Martí, and C. J. Oton \\ Valencia Nanophotonics Technology Center, Universidad Politécnica de \\ Valencia
}

\begin{abstract}
An accurate technique to characterize chromatic dispersion and its slope versus wavelength is reported. The method is based on a heterodyne Mach-Zehnder interferometer, which is immune to thermal phase noise by using a counterpropagating reference beam. Chromatic dispersion profiles are obtained over a broad wavelength region even in short waveguides with considerable loss. Conventional strip silicon waveguides as well as slotted geometries are considered. Theoretical simulations are also presented for comparison, which show good agreement with the experimental results.
\end{abstract}

\section{Introduction}

Transporting and processing high bitrate signals require a precise management of dispersion properties [1], [2]. Nonlinear effects are also very sensitive to, not only dispersion, but its exact dependence with wavelength, i.e., its higher order derivatives [1]. Chromatic dispersion measurement techniques have been reported by many authors. Examples of these are techniques like time-of-flight [3] or phase-shift methods [4]. Time-of-flight methods are based on the relative temporal delays measurement for pulses at different wavelengths and, in phaseshift methods, the input to output phase shift of a modulated signal is measured in order to obtain the group delay spectra. These dispersion characterization techniques were originally conceived for optical fibers, where propagation distance can be made very long in order to provide large delays. When the sample to measure is short, more precise interferometric techniques are needed like the ones reported in [5]-[10]. These techniques employ a Mach-Zehnder interferometer (MZI) or Michaelson interferometer, where the fringe positions provide the phase information. However, interferometers, especially if fiber-based, suffer from thermal fluctuations which introduce phase noise that randomly 
shifts the fringes. If the insertion loss of the sample is high, the measurement may require several seconds or minutes, which is a timescale where thermal phase noise can dramatically degrade the measurement.

In other references [11]-[13], waveguide dispersion is measured by making a fully- integrated unbalanced MZI. Scanning the wavelength one can extract dispersion by measuring the change of free-spectral range (FSR) of the fringes. However, some problems of this technique are i) this only provides discrete values of group index, ii) these measurements are also affected by thermal noise, and iii) the technique cannot be applied to a single straight waveguide, because an integrated MZI is needed.

In this paper, we propose a method for chromatic dispersion characterization with a fiber-based MZI using a technique which is immune to thermal phase noise. We describe how the proposed experimental setup compensates the fringe instability and we show continuous chromatic dispersion profiles obtained for different waveguide geometries together with theoretical calculations.

\section{Technique}

Fig. 1 shows the measurement apparatus. It is a fiber-based MZI, where one of the branches has an optical delay line (ODL), and the other has the waveguide sample. Acoustooptic modulators (AOMs) are also present in both branches, and they are used as frequency shifters. Their RF frequencies are set to 80 and $80.04 \mathrm{MHz}$, respectively, so that when the beams are recombined they produce $40 \mathrm{kHz}$ beatings which can be precisely measured with a lock-in amplifier. The lock-in also extracts the phase of the beatings, which is measured with respect to the phase of the beatings produced by a counterpropagating beam. This is carried out by amplifying the signal from the reference photodiode and sending it to the lock-in reference input. In this way, thermal fluctuations which affect both beams are cancelled out, and only the wavelength dependence of the phase is extracted during the sweep. A similar concept for stabilizing a MZI was reported in [14] for a distributed fiber sensor system. In principle, one could also use a copropagating beam as a reference, but that would require filters to separate the signal from the reference beam, thus a counterpropagating reference is easier to implement. On the other hand, if the lock-in amplifier in use can reach the $\mathrm{MHz}$ range, only one AOM would be necessary, as the beatings would have $80 \mathrm{MHz}$ frequency.

Let us obtain the equations that govern the phase response of this system. Frequency dependent propagation constant can be expanded in 


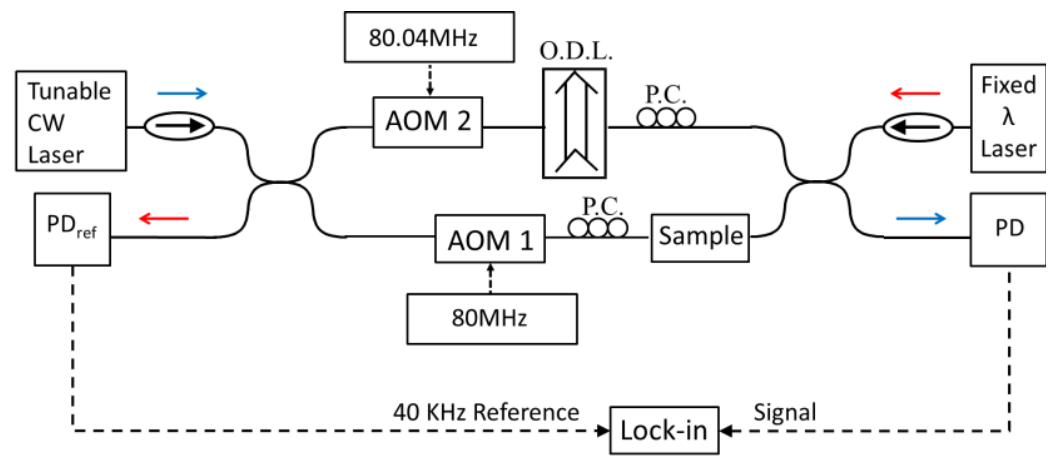

Fig. 1. Experimental setup. PC: Polarization controller, PD: Photo-detector, AOM: Acoustooptic modulator, ODL: Optical delay line. Solid lines denote fiber connections, and dashed lines, electrical connections. The ODL is pigtailed, but has a free-space delay inside. Isolators suppress the optical signals travelling toward the laser outputs.

Taylor series as follows [15]:

$$
\beta(\omega)=\mathrm{n}_{\mathrm{eff}}(\omega) \frac{\omega}{\mathrm{c}}=\beta_{0}+\beta_{1}\left(\omega-\omega_{0}\right)+\frac{1}{2 !} \beta_{2}\left(\omega-\omega_{0}\right)^{2}+\frac{1}{3 !} \beta_{3}\left(\omega-\omega_{0}\right)^{3}+\cdots
$$

where $\beta_{i}=\left(d^{i} \beta / d \omega^{i}\right)_{\omega=\omega_{0}}(\mathrm{i}=0,1,2 \ldots)$. The first three terms of the propagation constant can be expressed as

$$
\beta_{0}=\frac{\omega_{0}}{c} n_{e f f}\left(\omega_{0}\right), \quad \beta_{1}=\frac{n_{g}\left(\omega_{0}\right)}{c}, \quad \beta_{2}=\frac{-\lambda^{2}}{2 \pi c} D_{\lambda}
$$

where $n_{\text {eff }}$ is the effective index, $\beta_{1}$ is related to the group index $\left(n_{g}\right)$ and $\beta_{2}$ is the group velocity dispersion (GVD) parameter. Let us consider a MZI consisting of an homogeneous waveguide in one arm and a variable air path, e.g., an ODL, in the other branch. To compensate for the response of the system, two measurements with different lengths are necessary, the shortest one to be used as a reference. There are two possibilities to make this, as shown in Fig. 2. If the waveguide is homogeneous (no tapers or wider parts to facilitate the coupling) one can remove the sample to get the reference response [see Fig. 2(a)]. On the other hand, if the waveguide has section variations of considerable length, the reference must contain the same section variations, and only the length of the section under test must be different, as shown in Fig. 2(b).

In any case, we are interested in the phase difference with respect to the reference measurement, therefore the dispersion response of the AOMs, fibers, and waveguide coupling regions are all compensated. The response will only depend on $\mathrm{Ls}$, which is the extra waveguide length 
a)

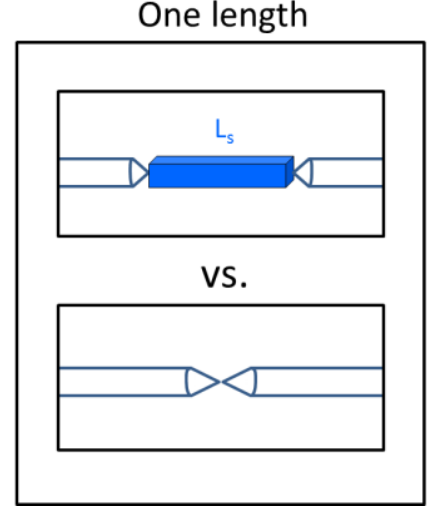

b)

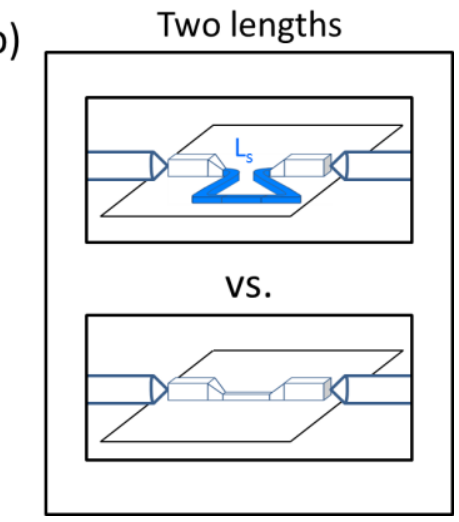

Fig. 2. Different measurement schemes, where the actual measurement is shown in the top panel and the reference in the bottom panel. (a) Only one length of homogeneous waveguide using a measurement with no sample as reference. (b) Two different lengths of a waveguide, using the shorter one as the reference.

between both measurements, and $\mathrm{La}_{\mathrm{a}}$ which is the extra air path added to the ODL to keep the MZI balanced.

If we define $\phi_{\mathrm{s}}$ as the phase added when increasing the waveguide length by $\mathrm{L}_{\mathrm{s}}$ and $\phi_{\mathrm{a}}$ as the phase added when increasing the ODL path by $\mathrm{L}_{\mathrm{a}}$, we have

$$
\begin{gathered}
\phi_{\mathrm{s}}(\omega)-\phi_{\mathrm{s}}\left(\omega_{0}\right)=\mathrm{L}_{\mathrm{s}}\left(\beta_{1} \Delta \omega+\frac{\beta_{2}}{2 !} \Delta \omega^{2}+\frac{\beta_{3}}{3 !} \Delta \omega^{3}\right) \\
\phi_{\mathrm{a}}(\omega)-\phi_{\mathrm{a}}\left(\omega_{0}\right)=\mathrm{L}_{\mathrm{a}} \frac{\Delta \omega}{\mathrm{c}}
\end{gathered}
$$

Hence, the phase difference between both branches $\Delta \phi=\phi_{s}-\phi_{a}$ is given by

$$
\Delta \phi(\omega)-\Delta \phi\left(\omega_{0}\right)=\left(\mathrm{L}_{\mathrm{s}} \beta_{1}-\frac{\mathrm{L}_{\mathrm{a}}}{\mathrm{c}}\right) \Delta \omega+\mathrm{L}_{\mathrm{s}}\left(\frac{1}{2 !} \beta_{2} \Delta \omega^{2}+\frac{1}{3 !} \beta_{3} \Delta \omega^{3}\right)
$$

Balancing the MZI consists of adjusting $L_{a}$ to make the first term in $\Delta \omega$ equal to zero. This is experimentally carried out by moving the ODL until the slope of the phase at the central wavelength becomes zero. Under these conditions, the group index of the waveguide under test will be given by 


$$
\mathrm{n}_{\mathrm{g}}=\mathrm{c} \cdot \beta_{1}=\frac{\mathrm{L}_{\mathrm{a}}}{\mathrm{L}_{\mathrm{s}}}
$$

The ODL must have a range which is long enough to cover the path difference between the reference sweep and the signal sweep. An ODL range of 500 ps would allow the characterization of devices up to that amount of delay, which corresponds to an approximate length of $37 \mathrm{~mm}$ (assuming a group index of 4), which is enough for most photonic integrated circuits.

Once the MZI is balanced, the terms from (5) in $\Delta \omega^{2}$ and $\Delta \omega^{3}$ can be obtained from a polynomial fit of the resulting curve. In principle, one could also obtain higher-order dispersion terms, but these terms would only become evident if lower-order terms are small. In our case, we have fitted the curves up to third-order.

Fig. 3 shows the effect of thermal phase drift. The red dashed curve shows the fluctuations of the signal beam phase with time without using the counterpropagating beam as a reference. In this case, the lock-in amplifier was referenced with the $40 \mathrm{kHz}$ beatings obtained from mixing the RF sources of the AOMs. The plot shows a noise level which would prevent a reliable measurement of the phase dependence on wavelength with a sweep that takes several seconds. On the other hand, the solid black curve shows the phase when referencing with the beatings produced by the counterpropagating reference beam. The fixed wavelength of the counterpropagating beam must be close to the propagating signal wavelength, as this way the phase noise is reduced to the minimum. For this reason, it was set to the central wavelength where the MZI is balanced. The phase noise is greatly reduced because thermal fluctuations of the optical paths of both branches equally affect both beams.

\section{Fabrication}

In this paper, we show the characterization of silicon-based waveguides with three different geometries, which we call Sample A, B and C.

Sample A and Sample B are strip waveguides patterned with deep-UV lithography. They have $2 \mu \mathrm{m}$ oxide buffer, $25 \mathrm{~mm}$ length, and $215 \mathrm{~nm}$ height, as shown in Fig. 4(a) and (b). Sample A corresponds to a TE polarization channel waveguide with $430 \mathrm{~nm}$ width. Sample B, used as TM polarization channel waveguide, has $487 \mathrm{~nm}$ width. The sidewall angle introduced in the manufacturing process is $8^{\circ}$ and $6^{\circ}$ for Sample A and Sample B, respectively. These parameters were extracted from SEM micrographs, and were fine-tuned within the experimental error range to optimize the fitting. Sample C is a $50 \%$ asymmetric slot waveguide 


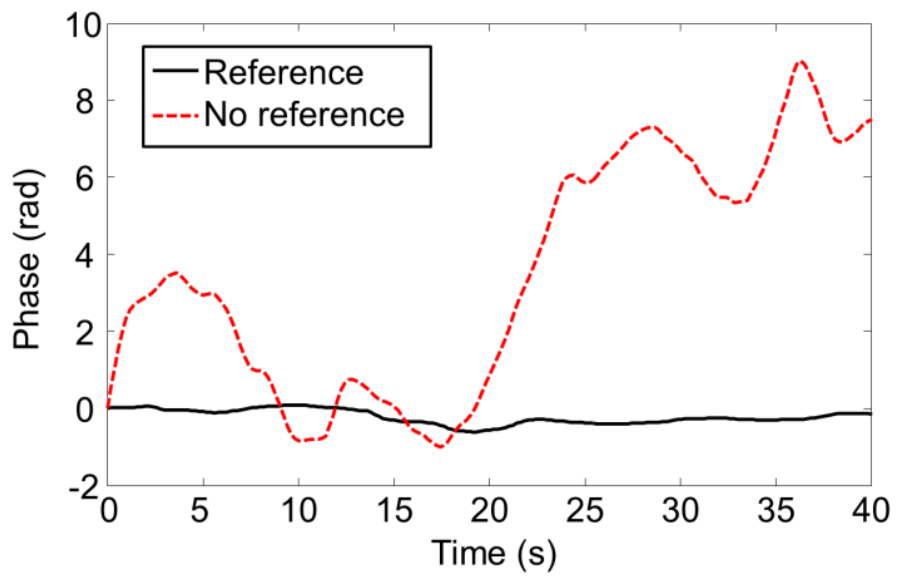

Fig. 3. Phase noise versus time when the measurement is unreferenced (red, dashed) and referenced with a counter-propagating beam at fixed wavelength (black, solid). Signal and reference wavelengths were 1550 and $1530 \mathrm{~nm}$, respectively. The phase noise is dramatically reduced by referencing.

patterned with electron-beam lithography. It has $3 \mu \mathrm{m}$ oxide buffer, 14 $\mathrm{mm}$ length, and $250 \mathrm{~nm}$ height. In asymmetric slot waveguides, the slot location is different than the geometrical center of the waveguide. We define the asymmetry degree as $2 s / w$ [16], where $s$ is the distance from the center of the waveguide to the center of the slot, in absolute value, and $w / 2$ is half of the total width of the waveguide, see Fig. 4(c). The waveguide consists of a $80 \mathrm{~nm}$ wide silica slot between two silicon rails with $5.5^{\circ}$ angled sidewalls. All samples are covered with silica using plasma-enhanced chemical vapor deposition (PECVD).

In samples A and B light was vertically coupled through $70 \mathrm{~nm}$-deep grating couplers. Total insertion loss was $24 \mathrm{~dB}$ for Sample A (TE) and 15 $\mathrm{dB}$ for Sample B (TM). On the other hand, for Sample C light was horizontally coupled using lensed fibers; total insertion loss was $54 \mathrm{~dB}$.

\section{Results}

Fig. 5(a) and (c) show the phase experimental measurement and its polynomial fit for Sample A and Sample B, respectively. From the fit for each sample, chromatic dispersion profiles are obtained, Fig. 5(b) and (d), where numerical simulations performed by using commercial 
a) Sample $A$

(TE)

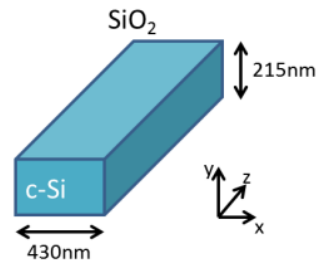

b) Sample B

(TM)

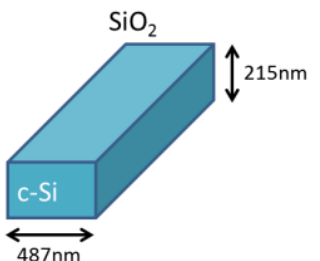

c)

Sample C

(TE)

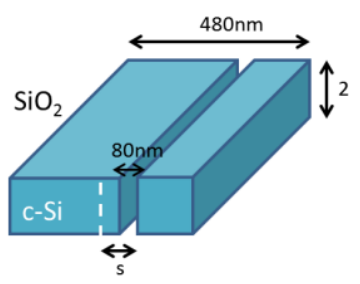

Fig. 4. Different measured waveguides. (a) $430 \mathrm{~nm}$ wide silicon strip for TE polarization. (b) $487 \mathrm{~nm}$ wide silicon strip for TM polarization. (c) Vertical slot waveguide. Waveguide parameters were extracted from SEM micrographs and were fine-tuned within the measurement error range to optimize the fitting.

software based on finite element method are also presented for comparison. In theoretical calculations, we compute the effective index, $\mathrm{n}_{\text {eff }}(\lambda)$, in a broad spectral range and by numerical differentiation the GVD parameter as a function of wavelength is obtained as $D_{\lambda}=-$ $(\lambda / c) d^{2} n_{\text {eff }} / d \lambda^{2}$. Material dispersion has been taken into account by considering the Sellmeier equation for both silicon and silica [17].

It can be seen that dispersion values and their slopes reasonably agree with the calculations; the small discrepancies are attributed to geometrical deviations of the fabricated device with respect to the simulation, which is assumed to be perfectly symmetric and homogeneous. In principle, there is no limitation on the dispersion measurable range. However, the minimum measurable dispersion would be determined by the phase noise, which depends on the experimental conditions. $\beta_{3}$ parameter is also obtained for both samples; for Sample A, we found an experimental value of $\beta_{3}=-0.0394 \mathrm{ps}^{3} / \mathrm{m}$ and a theoretical value of $\beta_{3}=-0.0392 \mathrm{ps}^{3} / \mathrm{m}$. In the case of Sample $B$, experimental $\beta_{3}=$ $0.019 \mathrm{ps}^{3} / \mathrm{m}$ and calculated $\beta_{3}=0.0076 \mathrm{ps}^{3} / \mathrm{m}$ values are obtained. Experimental group index values are 4.36 and 3.21 for TE polarization strip and TM polarization strip, respectively, while calculated values are 4.35 and 3.46, showing a good agreement too. Vertical slot waveguide experimental phase and its fitting are shown in Fig. 5(e). Fig. 5(f) shows the chromatic dispersion profile for this sample which presents a measured value of $\beta_{3}=-0.0939 \mathrm{ps}^{3} / \mathrm{m}$ and a calculated value of $\beta_{3}=-$ $0.0886 \mathrm{ps}^{3} / \mathrm{m}$. Experimental and theoretical values of group index for Sample C are 4.17 and 4.15 , respectively. 

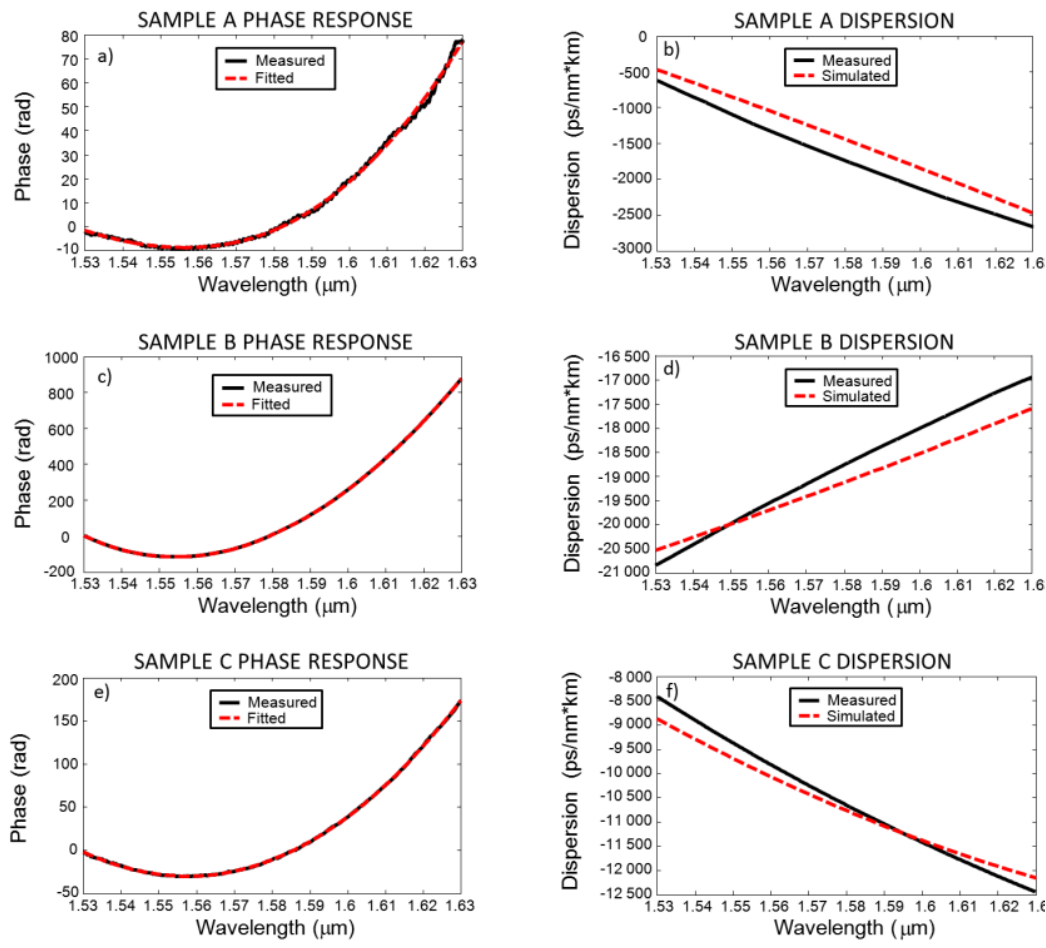

Fig. 5. Phase evolution and chromatic dispersion profiles for (a)-(b) TE polarization strip waveguide (Sample A), (c)-(d) TM polarization strip waveguide (Sample B) and (e)-(f) vertical slot waveguide (Sample C).

\section{Conclusion}

A novel method for chromatic dispersion measurement in nanophotonic waveguides is proposed and demonstrated. By measuring the phase evolution versus wavelength of the waveguides in a MZI, the chromatic dispersion and its slope can be determined. The technique is immune to fringe instability and allows the characterization of integrated waveguides with high insertion loss. We have performed an accurate dispersion characterization of conventional strip waveguides for both TE and TM polarizations as well as vertical slot waveguide. The experimental results were in close agreement with those obtained from simulation. 


\section{References}

[1] I. Walmsley, L. Waxer, and C. Dorrer, 'The role of dispersion in ultrafast optics', Review of Scientific Instruments 72, 1, 1-29, 2001.

[2] L. Zhang, Q. Lin, Y. Yue, R. G. Beausoleil, and A. E. Willner, 'Silicon waveguide with four zero-dispersion wavelengths and its application in on-chip octave-spanning supercontinuum generation', Optics Express 20, 2, 1685-1690, 2012.

[3] L. Cohen and C. Lin, 'A universal fiber-optic (UFO) measurement system based on a near-IR Raman laser', IEEE Journal of Quantum Electronics, QE-14, 11, 855-859, 1978.

[4] J. Hult, R. S. Watt, and C. F. Kaminski, 'Dispersion measurement in optical fibers using supercontinuum pulses', Journal of Lightwave Technology 25, 3, 820-824, 2007.

[5] L. Thevenaz, J. P. Pellaux, and J. P. Von Der Weid, 'All-fiber interferometer for chromatic dispersion measurements', Journal of Lightwave Technology 6, 1, 1-7, 1988.

[6] H. T. Shang, 'Chromatic dispersion measurement by white-light interferometry on metre-length single-mode optical fibres', Electronic Letters 17, 17, 603-605, 1981.

[7] J. Y. Lee and D. Y. Kim, 'Versatile chromatic dispersion measurement of a single mode fiber using spectral white light interferometry', Optics Express 14, 24, 11 608-11 615, 2006.

[8] H. K. Tsang, C. S. Wong, T. K. Liang, I. E. Day, S. W. Roberts, A. Harpin, J. Drake, and M. Asghari, 'Optical dispersion, two-photon absorption and self-phase modulation in silicon waveguides at $1.5 \mu \mathrm{m}$ wavelength', Applied Physics Letters 80, 3, 416-418, 2002.

[9] C. Turner, C. Manolatou, B. S. Schmidt, M. Lipson, M. A. Foster, J. E. Sharping, and A. L. Gaeta, 'Tailored anomalous group-velocity dispersion in silicon channel waveguides', Optics Express 14, 10, 4357-4362, 2006.

[10] W. Ding, C. Benton, A. V. Gorbach, W. J. Wadsworth, J. C. Knight, D. V. Skryabin, M. Gnan, M. Sorrel, and R. M. De la Rue, 'Solitons and spectral broadening in long silicon-on-insulator photonic wires', Optics Express 16, 5, 3310-3319, 2008.

[11] E. Dulkeith, F. Xia, L. Schares, W. M. J. Green, and Y. A. Vlasov, 'Group index and group velocity dispersion in silicon on- insulator photonic wires', Optics Express 14, 9, 3853-3863, 2006. 
[12] Y. A. Vlasov, M. OBoyle, H. F. Hamann, and S. J. McNab, 'Active control of slow light on a chip with photonic crystal waveguides', Nature, 438, 65-69, 2005.

[13] D. T. H. Tan, K. Ikeda, P. C. Sun, and Y. Fainman, 'Group velocity dispersion and self-phase modulation in silicon nitride waveguides', Applied Physics Letters 96, 6, 61101-1-61101-3, 2010.

[14] J. Snoddy, Y. Li, F. Ravet, and X. Bao, 'Stabilization of electro-optic modulator bias voltage drift using a lock-in amplifier and a proportional-integral-derivative controller in a distributed Brillouin sensor system', Applied Optics 46, 9, 1482-1485, 2007.

[15] G. P. Agrawal, 'Nonlinear Fiber Optics', 3rd Edition, New York Academic, 2001.

[16] S. Mas, J. Caraquitena, J. V. Galán, P. Sanchis, and J. Martı', 'Tailoring the dispersion behavior of silicon nanophotonic slot waveguides', Optics Express 18, 20, 20 839-20 844, 2010.

[17] B. Tatian, 'Fitting refractive-index data with the Sellmeier dispersion formula', Applied Optics 23, 24, 4477-4485, 1984. 


\title{
Paper C
}

\section{“Group Delay and Dispersion Tailoring in Nonadiabatic Tapered Fibers"}

\author{
S. Mas, J. Palací, and J. Martí \\ Submitted to IEEE Journal of Selected Topics in Quantum \\ Electronics
}




\title{
Group delay and dispersion tailoring in nonadiabatic tapered fibers
}

\author{
S. Mas,,$^{1, *}$ J. Palací, ${ }^{2}$ and J. Martí1 \\ ${ }^{1}$ Valencia Nanophotonics Technology Center, Universitat Politècnica de \\ València, 46022 Valencia, Spain \\ ${ }^{2}$ Luz Wavelabs S.L., Parque Científico Universidad Carlos III, 28919 \\ Madrid, Spain
}

\begin{abstract}
The dispersion profile of a nonadiabatic tapered singlemode fiber is characterized and dynamically tuned. Its group delay and dispersion parameters are measured and compared to those of a standard singlemode fiber. The dispersion profile can be tuned by introducing a phase shift through mechanical stretching. Coarse tuning is also obtained by varying the surrounding medium of the tapered fiber. Dispersion values up to $700 \mathrm{ps} / \mathrm{nm} \cdot \mathrm{km}$ in nonadiabatic tapered fibers are obtained for the first time. Dynamic tuning exposed here can be very useful in applications such as nonlinearities or soliton generation.
\end{abstract}

Chromatic dispersion is one of the limiting effects in optical communications systems. It broadens optical pulses leading to degradation in the quality of the system, not only increasing the noise level in analogical systems but also inducing Inter Symbol Interference (ISI) in digital systems [1,2]. The latter reduces the bitrate-distance product, this is the amount of data that can be transmitted per time and space through optical links. Besides, several functionalities such as nonlinear generation [3], broadband optical parametric gain [4] and soliton generation [5] are highly dependent on the dispersion characteristics of the device. Therefore, an accurate management of the chromatic dispersion profile of devices and systems is required. Chromatic dispersion can be split into material dispersion and 
waveguide dispersion. Dispersion tailoring is usually performed by varying the structural and geometrical parameters of the device [6-8], which allows for more control on their response as opposed to material engineering which usually requires the fabrication of different devices using different materials.

Tapered fibers consist of a narrow waist located between two transition regions [9]. This structure is obtained by exposing a standard single-mode fiber to a heating and stretching process [10]. Depending on the length of the transition region two types of tapered fibers can be distinguished: in adiabatic tapers these regions are long enough to allow the fundamental mode to propagate normally through the taper; however, non-adiabatic tapers are abrupt and the fundamental mode experiences higher-order mode coupling in the tapered regions. Biconical tapered fibers have been employed in several functionalities such as sensing [11], super-continuum generation [12] and pulse shaping [13]. The great development of tapered fibers lies in the simplicity in which conventional propagation characteristics of standard optical fibers can be modified.

In this context, dispersion tailoring in adiabatic tapered fibers have been demonstrated by the modification of geometrical parameters such as the waist diameter [14] and structural parameters such as the refractive index of the outer medium $[15,16]$. However, if the tapered fiber is immersed in different liquids to modify its surrounding medium, a very careful cleaning must be carried out after the measurements in order to return to the starting state of the taper. Also, dispersion engineering just by means of the variation of geometrical parameters in the manufacturing process presents a more static behavior. This paper proposes using non-adiabatic tapered fibers to perform dynamic chromatic dispersion tailoring in a wide bandwidth by mechanically stretching the fiber. This stretching introduces a controlled phase shift which affects the spectral response, varying the dispersion characteristics of the fiber in a simple and dynamic way. Further control on the dispersion response is demonstrated by modifying the refractive index (RI) of the outer medium without immersing the tapered fiber in fluids.

Fig. 1 shows the profile of a non-adiabatic biconical tapered fiber with waist of diameter $\rho$ and length $\mathrm{L}_{\mathrm{w}}$ located between two transition regions of length $\mathrm{T}_{\mathrm{t}}$. Most of the energy from the fundamental mode that is injected through the input taper splits into the cladding fundamental mode and a higher-order cladding mode. These modes propagate through the waist experiencing different effective propagation distances that depend on their mode effective indexes [17]. These modes interfere 
at the output transition region, leading to an interference fringe pattern in the frequency domain, similar to that of a Mach-Zehnder interferometer (MZI). The phase of the transference function of the tapered fiber follows [13]

$$
\varphi(\lambda) \sim \sin \left(\frac{2 \pi}{\lambda} \cdot L_{w} \cdot\left(n_{e f f 1}-n_{e f f 2}\right)+\varphi_{0}\right)
$$

where $\Delta n_{\text {eff }}=n_{\text {eff1 }}-n_{\text {eff } 2}$ accounts for the difference between the effective indexes of the waist modes, $\lambda$ is the vacuum wavelength and $\varphi_{0}$ is the initial phase. According to this expression, variations in waist length [18] and effective indexes [19,20] of the modes directly influence the spectral response of the taper. Group delay and chromatic dispersion are related to the phase by the following relations

$$
\begin{array}{r}
\tau_{\mathrm{g}}=\frac{\partial \varphi}{\partial \omega} \sim \frac{L_{w}}{\mathrm{c}} \cdot \Delta n_{\text {eff }} \cdot \cos \left(\frac{2 \pi}{\lambda} \cdot L_{w} \cdot \Delta n_{e f f}+\varphi_{0}\right) \\
D=\frac{d}{d \lambda}\left(\frac{1}{\tau_{g}}\right) \sim \frac{-2 \pi c}{\lambda^{2}} \cdot \tan \left(\frac{2 \pi}{\lambda} \cdot L_{w} \cdot \Delta n_{e f f}+\varphi_{0}\right) \cdot \sec \left(\frac{2 \pi}{\lambda} \cdot L_{w} \cdot \Delta n_{e f f}+\varphi_{0}\right)
\end{array}
$$

Thus, by varying the phase difference of the interference pattern, group delay and chromatic dispersion of the tapered fiber can be modified. According to Eq.2, maximum values in the phase transference function become minimum values in the group delay profile and vice versa. Please note that this expression accounts for the effect of the tapered fiber but not for the effect of the waveguide and material of the optical fiber. Eq.3, shows how dispersion becomes zero at local maximums in the group delay and maximum absolute values at the local minimums.

Figure 2 shows the transmission response of a non-adiabatic

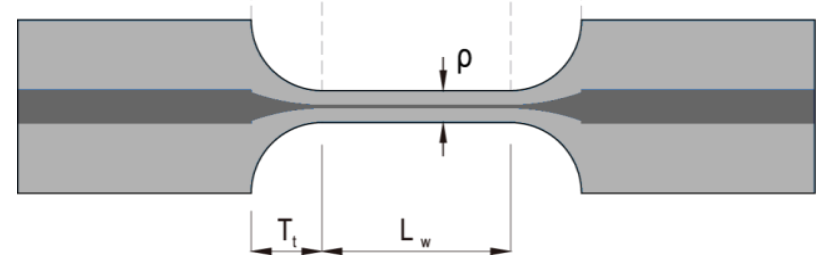

Fig. 1. Biconical tapered fiber. 


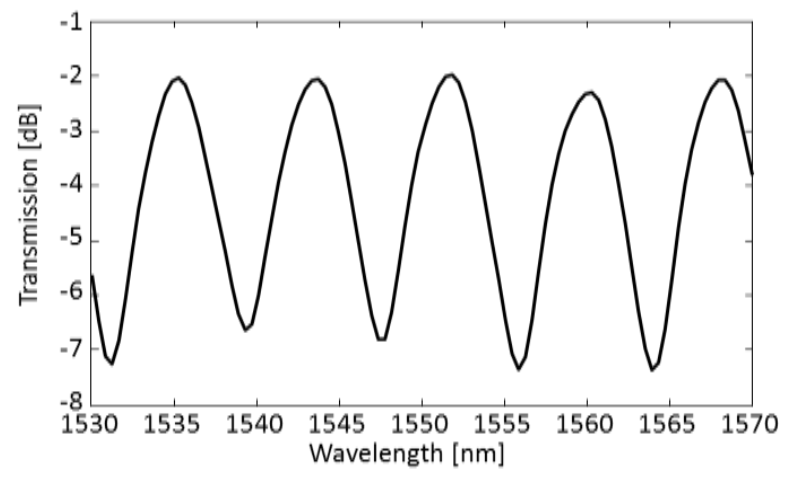

Fig. 2. Experimental transmission response of a biconical tapered fiber.

tapered fiber manufactured in a standard single-mode fiber (SSMF) with parameters $\mathrm{T}_{\mathrm{t}}=1 \mathrm{~mm}, \mathrm{~L}_{\mathrm{w}}=13 \mathrm{~mm}$ and $\rho=18 \mu \mathrm{m}$, obtained using an Optical Network Analyzer (ONA) from Advantest. Frequency modulation and fiber index were set to $3 \mathrm{GHz}$ and 1.45, respectively. The length of the whole section of fiber is $L=79.5 \mathrm{~cm}$. As can be seen in its fringe pattern, the taper presents a free spectral range (FSR) value of $8.2 \mathrm{~nm}$ and a visibility of approximately $5 \mathrm{~dB}$. The taper was set in a translation stage by fixing its sides using two drops of glue in contact with the cladding. Group delay and chromatic dispersion characteristics of the tapered fiber as a function of wavelength were measured by using the ONA with the same configuration parameters, and the obtained results are illustrated in Figure 3. A SSMF section of fiber with the same total length $\mathrm{L}$ as the tapered segment was also characterized for comparison purposes in a $40 \mathrm{~nm}$ bandwidth centered at $1550 \mathrm{~nm}$. As can be seen, inserting the taper clearly modifies the conventional propagation characteristics of the standard fiber, resulting in a periodic behavior in its group delay response. The periodicity of the group delay profile corresponds with the FSR of the transmission response. Likewise, the chromatic dispersion profile is also modified as can be seen in Fig. 3(b). Both group delay and dispersion behavior are in agreement with the results predicted by Eq. 2 and Eq. 3. In this wavelength span conventional fibers show an almost constant value of $18 \mathrm{ps} / \mathrm{nm} \cdot \mathrm{km}$. Much higher values of approximately $500 \mathrm{ps} / \mathrm{nm} \cdot \mathrm{km}$ are observed in the 
periodic dispersion profile at the minimum values of the group delay as expected from Eq. 3.

Dynamic tuning is observed by stretching the tapered fiber to three different points of elongation, $\mathrm{L}_{1}=0 \mu \mathrm{m}, \mathrm{L}_{2}=250 \mu \mathrm{m}$ and $\mathrm{L}_{3}=500 \mu \mathrm{m}$, respectively. The starting point, $\mathrm{L}_{1}$, is the same elongation state as the one previously compared with the untapered fiber in Figure 3. Figure 4 shows the optical transmission function of the stretched tapered fiber for the three elongation points. As can be seen, the modification of $\mathrm{L}_{\mathrm{w}}$ induces changes in the interference pattern, leading to a spectral shift, as predicted from Eq. 1. Linear wavelength shifts of $0.2 \mathrm{~nm}$ and $0.4 \mathrm{~nm}$ are observed when the taper fiber is elongated from $\mathrm{L}_{1}$ to $\mathrm{L}_{2}$ and $\mathrm{L}_{3}$, respectively. Figure 5 shows the group delay and chromatic dispersion profiles obtained for these elongation points. Group delay profiles experience a shift to higher wavelengths when the tapered fiber is stretched. Considering the dispersion peaks nearest to the telecommunications wavelength of $1550 \mathrm{~nm}$, elongation point $\mathrm{L}_{1}$ presents its maximum value of $427 \mathrm{ps} / \mathrm{nm} \cdot \mathrm{km}$ at $1547.9 \mathrm{~nm}, \mathrm{~L}_{2}$ maximum value is $450 \mathrm{ps} / \mathrm{nm} \cdot \mathrm{km}$ at $1548.52 \mathrm{~nm}$ and $\mathrm{L}_{3}$ peak of 480 $\mathrm{ps} / \mathrm{nm} \cdot \mathrm{km}$ is located at $1549.06 \mathrm{~nm}$. To sum up, mechanical stretching of the structure allows for fine tuning of its dispersion profile. Also, the modification of the dispersion profile shape provides periodic zero dispersion wavelengths, as shown in Fig. 5(b) which can be useful in applications requiring dispersion compensation or propagation in nondispersive media.

Taking advantage of the evanescent part of the fields propagating through the waist the characteristics of the tapered fiber can be easily modified. The effective indexes of the propagating modes can be varied by putting the tapered fiber in contact with a support surface as shown in [21]. This allows the modification of the refractive index of the surrounding medium leading to the variation of the propagation characteristics of the tapered fiber. The transmission response of a tapered fiber with the same geometrical parameters $L_{w}, T_{t}$ and $\rho$ as the previously analyzed but located on-surface is depicted in Fig. 6. As can be seen, its visibility and FSR are approximately $7 \mathrm{~dB}$ and $8.2 \mathrm{~nm}$, respectively, leading to more abrupt resonances in comparison with the optical response of the over-surface tapered fiber. The contact between the support surface and the tapered fiber leads to scattering losses and a wavelength shift due to the different index of the surrounding medium. 

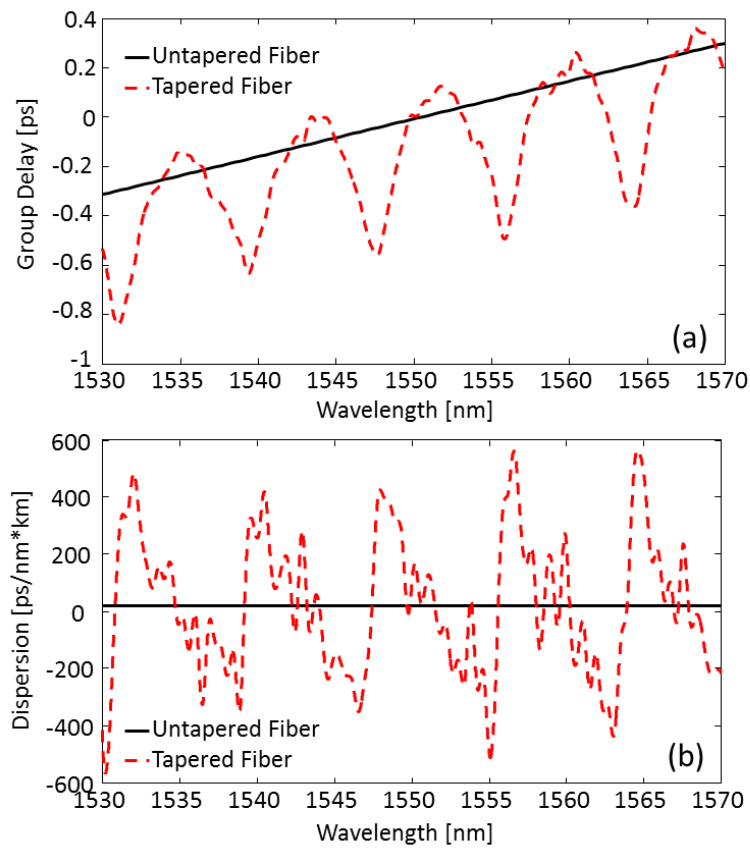

Fig. 3. (a) Group delay and (b) chromatic dispersion of a single mode standard fiber with and without tapering it.

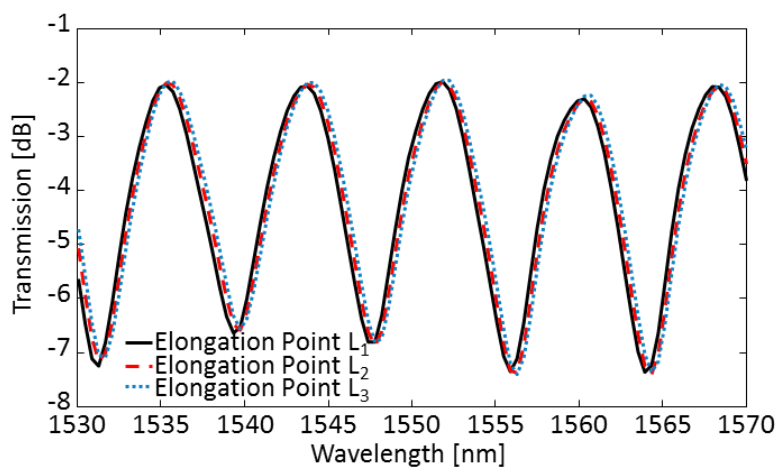

Fig. 4. Experimental response of a stretched biconical tapered fiber considering three different elongation points. 

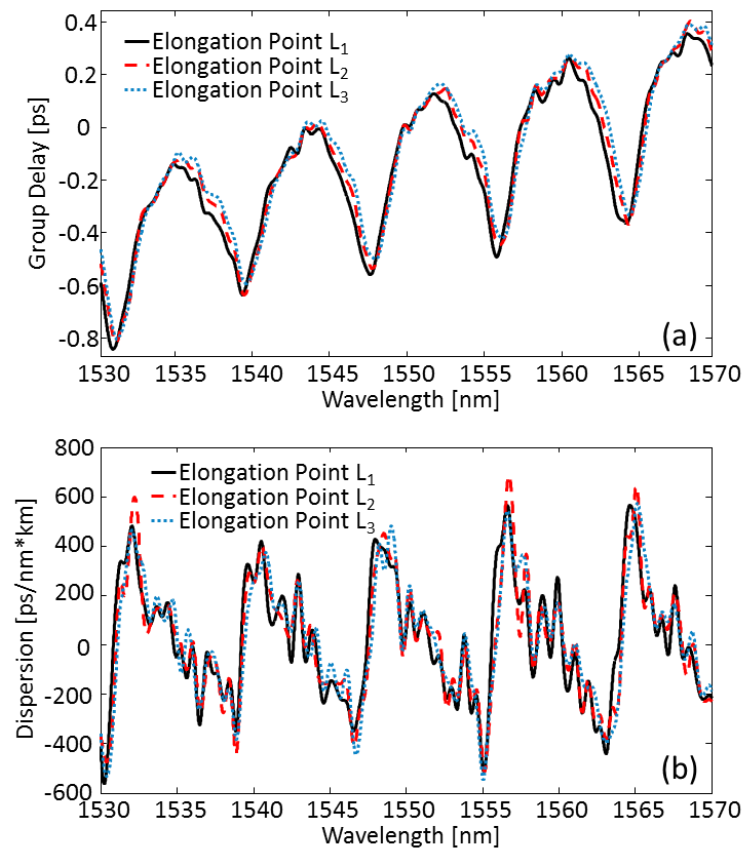

Fig. 5. (a) Group delay and (b) chromatic dispersion of a stretched tapered fiber considering three different points of elongation.

Losses cause the modes travelling along the waist to present more similar amplitudes, thus reducing the residual power under destructive interference resulting in more abrupt resonances in its transference function. Group delay and chromatic dispersion characteristics are shown in Fig. 7 where the results obtained for the over-surface tapered fiber are also included for comparison purposes. As can be seen, group delay profile is shifted approximately the half of the FSR, i.e. around 4 $\mathrm{nm}$, as well as its chromatic dispersion profile. Furthermore, both profiles present higher visibilities than those of the over-surface taper, which is in agreement with the higher visibility exposed in the transmission response in Fig. 6. Maximum dispersion peaks of approximately $700 \mathrm{ps} / \mathrm{nm} \cdot \mathrm{km}$ can be achieved, which is more than 40 times the dispersion value present in a SSMF at the considered bandwidth.

In conclusion, we have proposed a novel technique to implement dynamic dispersion tailoring based on non-adiabatic fiber tapers. It has 


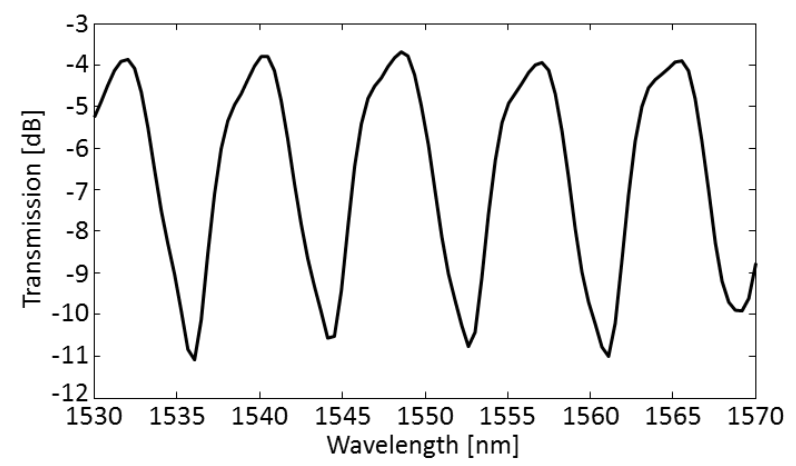

Fig. 6. Experimental transmission response of an on-surface tapered fiber.
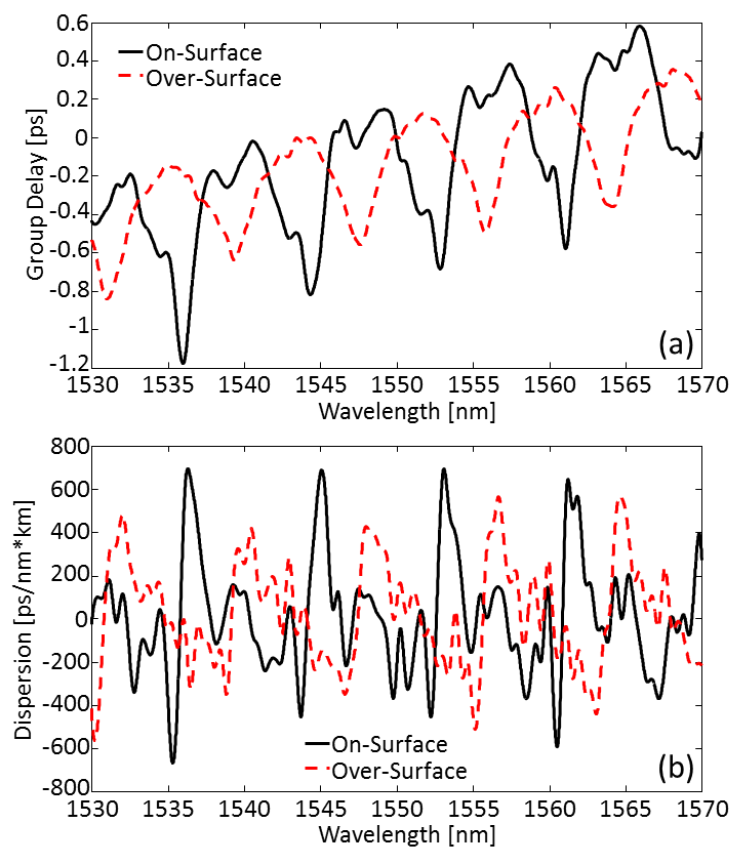

Fig. 7. (a) Group delay and (b) chromatic dispersion of On-surface and Over-surface tapered fibers. 
been fabricated and its amplitude, group delay and chromatic dispersion responses measured and compared with those of an untapered fiber with the same length and characteristics. The interferometric response of the structure has been controlled by mechanical stretching and its dispersion characteristics measured as a function of the elongation. This technique can be applied to carry out fine-tuning dispersion engineering in applications such as nonlinearities or soliton propagation where chromatic dispersion characteristics are crucial. In addition, pulse shaping and dispersion compensation modules can benefit from this tailoring technique. We obtained stable and repeatable measurements, thanks in part to the robustness of the relatively large tapers employed. The approach is not only scalable and simple, but also allows for a dispersion tailoring in a wide bandwidth. Combined ways of dispersion tailoring in tapered fibers may be considered to obtain a higher level of dispersion control. Results by locating the tapered fiber in contact with a support surface are also exposed, showing that dispersion can be tailored from the conventional value of $17 \mathrm{ps} / \mathrm{nm} \cdot \mathrm{km}$ at $1550 \mathrm{~nm}$ to more than $700 \mathrm{ps} / \mathrm{nm} \cdot \mathrm{km}$. Therefore, effective index variations along with the stretching process presented here can be combined to implement dynamic dispersion tailoring.

\section{References}

[1] G. P. Agrawal, 'Fiber-Optics Communication Systems', Third Edition, Wiley-Interscience, 2002.

[2] G.P. Agrawal, 'Nonlinear Fiber Optics', Third Edition, Academic Press, 2001.

[3] R.W. Boyd, 'Nonlinear Optics', Third Edition, Academic Press, 2007.

[4] M.A. Foster, A.C. Turner, J.E. Sharping, B.S. Schmidt, M. Lipson, and A.L. Gaeta, 'Broadband optical parametric gain on a silicon photonic chip', Nature 441, 960-963, 2006.

[5] L. Yin, Q. Lin, and G.P. Agrawal, 'Dispersion tailoring and soliton propagation in silicon waveguides', Optics Letters 31, 9, 1295-1297, 2006.

[6] T. D. Engeness, M. Ibanescu, S.G. Johnson, O. Weisberg, M. Skorobogatiy, S. Jacobs, and Y. Fink, 'Dispersion tailoring and compensation by modal interactions in OmniGuide fibers', Optics Express 11, 10, 1175-1196, 2003. 
[7] A.C. Turner, C. Manolatou, B.S. Schmidt, M. Lipson, M.A. Foster, J.E. Sharping, and A.L. Gaeta, 'Tailored anomalous group-velocity dispersion in silicon channel waveguides', Optics Express 14, 10, 4357-4362, 2006.

[8] S. Mas, J. Caraquitena, J.V. Galán, P. Sanchis, and J. Martí, 'Tailoring the dispersion behavior of silicon nanophotonic slot waveguides', Optics Express 18, 20, 20839-20844, 2010.

[9] K. Jedrzejewski, 'Biconical fused taper - a universal fibre devices technology', Opto-Electronics Review 8, 2, 153-159, 2000.

[10] J. Villatoro, D. Monzón-Hernández, and E. Mejía, 'Fabrication and modeling of uniform-waist singlemode tapered optical fiber sensors', Applied Optics 42, 13, 2278-2283, 2003.

[11] D. Monzón-Hernández, and J. Villatoro, 'High-resolution refractive index sensing by means of a multiple-peak surface plasmon resonance optical fiber sensor', Sensors and Actuators B 115, 227 231, 2006.

[12] Z. Wang, H. Sone, Y. Tsuji, and M. Imai, 'Spectral Phase and Intensity Evolutions of Supercontinuum Generation in a Biconical Tapered Fiber', Optical Review 14, 2, 81-85, 2007.

[13] S. Mas, J. Palací, P. Pérez-Millán, S. Lechago, D. Monzón-Hernández, and J. Martí, 'All-fiber processing of terahertz-bandwisth signals based on cascaded tapered fibers', Optics Letters 38, 23, 4954-4957, 2013.

[14] R. Zhang, X. Zhang, D. Meiser, and H. Giessen, 'Mode and group velocity dispersion evolution in the tapered region of a single-mode tapered fiber', Optics Express 12, 24, 5840-5849, 2004.

[15] R. Zhang, J. Teipel, X. Zhang, D. Nau, and H. Giessen, 'Group velocity dispersion of tapered fibers immersed in different liquids', Optics Express 12, 8, 1700-1707, 2004.

[16] C.M.B. Cordeiro, W.J. Wadsworth, T.A. Birks, and P.St.J. Russell, 'Engineering the dispersion of tapered fibers for supercontinuum generation with a $1064 \mathrm{~nm}$ pump laser', Optics Letters 30,15, 19801982, 2005.

[17] M.I. Zibaii, H. Latifi, Z. Saeedian, and Z. Chenari, 'Nonadiabatic tapered optical fiber sensor for measurement of antimicrobial activity of silver nanoparticles against Escerichia coli', Journal of Photochemistry and Photobiology B: Biology 135, 55-64, 2014.

[18] K. Q. Kieu, and M. Mansuripur, 'Biconical Fiber Taper Sensors', IEEE Photonics Technology Letters 18, 21, 2239-2241, 2006. 
[19] G. Cohoon, C. Boyter, M. Errico, K. Vandervoort, and E. Salik, 'Enhancing sensitivity of biconical tapered fiber sensors with multiple passes through the taper', Optical Engineering 49, 034401034401-5, 2010.

[20] W. B. Ji, H. H. Liu, S. C. Tjin, K. K. Chow, and A. Lim, 'Ultrahigh Sensitivity Refractive Index Sensor Based on Optical Microfiber', IEEE Photonics Technology Letters 24, 22, 1872-1874, 2012.

[21] S. Mas, J. Martí, and J. Palací, 'Biconical Tapered Fibers Manipulation for Refractive Index and Strain Sensing Applications', IEEE Sensors Journal 15, 3, 1331-1335, 2015. 


\section{Paper D}

\section{"All-Fiber Processing of Terahertz- Bandwidth Signals Based on Cascaded Tapered Fibers"}

S. Mas, J. Palací, P. Pérez-Millán, S. Lechago, D. MonzónHernández, and J. Martí

Optics Letters, Volume 38, Number 23, pp. 4954-4957, 2013. 


\title{
All-fiber processing of terahertz- bandwidth signals based on cascaded tapered fibers
}

\author{
S. Mas, ${ }^{1, *}{ }^{*}$. Palací, ${ }^{1}$ P. Pérez-Millán, ${ }^{2}$ S. Lechago, ${ }^{1}$ D. Monzón- \\ Hernández, ${ }^{3}$ and J. Martí ${ }^{1}$ \\ ${ }^{1}$ Valencia Nanophotonics Technology Center, Universitat Politècnica de \\ València, 46022 Valencia, Spain \\ ${ }^{2}$ Dpto. de Física Aplicada - ICMUV, Universitat de València, 46100 \\ Burjassot, Spain \\ ${ }^{3}$ Centro de Investigaciones en Óptica A.C., 37150 Guanajuato, México
}

\begin{abstract}
Tapered single-mode fibers are employed to perform dynamic pulse shaping in a bandwidth of several terahertz. The transfer function of cascaded biconical tapers is controlled by introducing a phase shift into one of them through mechanical stretching. It is a simple and low-cost technique with potential to process signals with bandwidths as large as those allocated by standard optical fiber while introducing little degradation. Femtosecond pulses are shaped to prove the concept.
\end{abstract}

Optical fiber is a versatile and excellent transmission medium employed not only in data distribution but also in signal processing. Different applications from sensing to imaging and pulse shaping have been implemented taking advantage of its capabilities [1]. Fibers with different properties can be obtained through material doping and the design of their geometry, allowing for different functionalities, such as dispersion compensation, increased nonlinear effects, and polarizationdependent behavior [2]. In the same direction, biconical tapered fibers emerged as a simple way to modify the properties of standard fiber by reducing its transversal section through heating and mechanical 
stretching along the propagation axis [3-5]. These consist of a uniform waist allocated between conical transition regions and are often classified according to the type of transition; nonadiabatic for abrupt ones, in which the fundamental mode of the untapered fiber couples not only to the core of the waist but also to the air-cladding interface, and adiabatic tapers, with more gradual transitions that do not introduce higher order coupling. Biconical tapers have been used in sensing [6,7], supercontinuum generation [8,9], directional coupling $[10,11]$, chromatic dispersion compensation [12], and fiber laser mode-locking [13]. Nonadiabatic tapers are particularly interesting in the fabrication of all-fiber temperature, stress, and refractive index sensors since light traveling through the outer interface of the fiber interacts with the external medium before being recoupled into the fundamental mode of the fiber.

Ultrashort optical pulses are of widespread use in different applications, such as spectroscopy, metrology, and communications [14]. Particular pulse shapes are required by some of them; e.g., to fit certain spectral masks in communications [15] and to locally increase the power in terahertz spectroscopy [16]. To accomplish this, pulse shaping techniques with bandwidths capable of allocating femtosecond pulses are necessary. Most of these techniques rely on dispersion to gain separate access to the different spectral components of the pulse, either in space [17], or in time [18], for free-space and fiber-based solutions, respectively. These approaches provide good performance but require careful dispersion compensation. This Letter proposes using fiber tapers to perform dynamic shaping of light in a bandwidth as large as that of standard optical fiber. One of the two fiber tapers, in series, is mechanically stretched to introduce a controlled phase shift that affects the cascaded spectral response. The processed signals experience little dispersion because of the reduced length of the tapers. This makes the technique optimal for the processing of signals of very large bandwidth without relying on dispersive effects that may be difficult to compensate. The approach is highly scalable and compatible with fiber-based systems.

Figure 1 shows the profile of a biconical tapered fiber with a waist of diameter $\rho$ and length $L_{w}$ between two exponential coupling regions of length $T_{t}$ [19]. Modes propagating through the waist experience different propagation distances, depending on their effective index, $n_{\text {effi }}$ for the $i$ th mode. This results in a fringe pattern in the frequency domain, similar to that of Mach-Zehnder interferometers (MZI), once they interfere with their delayed replicas after recoupling into the fundamental standard fiber mode $\left(n_{S S M F}\right)$. For the tapers considered in this work, most of the energy injected into the taper splits in two modes that propagate separately in the waist. The resulting transference function of the 


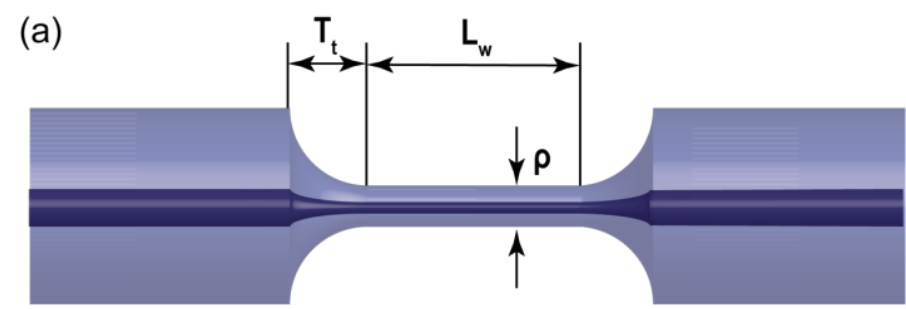

(b)

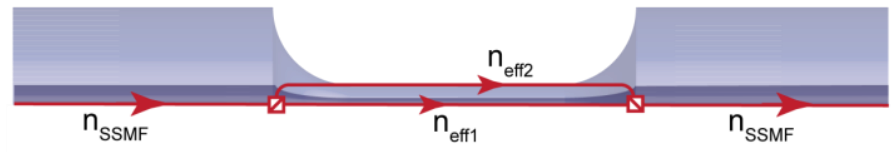

Fig. 1. (a) Biconical tapered fiber and (b) scheme of the interferometric phenomenon along the tapered fiber.

optical intensity (TF) is therefore expected to be as follows:

$$
\mathrm{TF} \sim \sin \left(\mathrm{k}_{0} \mathrm{~L}_{\mathrm{W}}\left(\mathrm{n}_{\mathrm{eff} 1}-\mathrm{n}_{\text {eff } 2}\right)+\varphi_{\mathrm{o}}\right)
$$

where $k_{0}$ and $\varphi_{0}$ are angular wavenumber in vacuum and phase offset, respectively. Variations in waist length [20] and effective indexes [21, 22] of the modes directly influence the spectral response of the taper. Although this has been extensively exploited in sensing applications, we turn around the concept by controlling the taper parameters to modify its TF. Assuming that the taper length is stretched by an amount $\Delta \mathrm{L}_{w}$, its spectral response will shift $\Delta \omega$ in optical frequency. According to Eq. (1), both variables follow the relation

$$
\frac{\Delta \omega}{\omega}=\frac{\Delta \mathrm{L}_{\mathrm{W}}}{\mathrm{L}_{\mathrm{W}}}
$$

which shows that small increases in the taper length introduce considerable frequency shifts.

Figure 2 shows the transmission response of two non-adiabatic fiber tapers that were fabricated with parameters $T_{t}=1 \mathrm{~mm}, L_{w}=13 \mathrm{~mm}$ and $\rho=18 \mu \mathrm{m}$, obtained using a tunable continuous-wave laser and a power meter. The fabrication technique consists of a small flame set in a three-axis-translation stage that allows us to move the flame consistently and accurately so as to ensure that the fiber is uniformly heated while the fiber is being stretched [5]. Deviations in this process led to different 
free-spectral ranges (FSR) of $8.3 \mathrm{~nm}$ and $7.25 \mathrm{~nm}$. Commercial simulation software was employed to compute the different modes propagating in the waist as well as their effective indexes, leading to

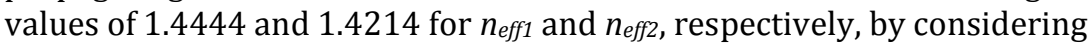
standard refractive index values for core and cladding at $1.55 \mu \mathrm{m}$. The experimental measurements agree well with these values while the quasi-sinusoidal transfer function confirms the two modes approximation. The fabricated tapers show insertion losses of $2 \mathrm{~dB}$, which are attributed to radiation in the transition sections as well as to propagation in the waist and transitions between the fibers. The second taper was stretched after fixing its sides onto a translation stage by means of two drops of glue in contact with the cladding of the untapered fiber and separated approximately $3.5 \mathrm{~cm}$. Results are shown in Fig. 2(b) where the starting point is the solid black line and the response shifts to lower wavelengths for increasing lengths, as expected from Eq. (1). Wavelength shifts of $1.5,2.8$ and $6.3 \mathrm{~nm}$ were measured. These values correspond with theoretical elongations of 12, 23 and $53 \mu \mathrm{m}$ for frequencies in the telecom wavelength of $1550 \mathrm{~nm}$, respectively. Small amplitude changes were observed for different stretching conditions. We attribute these variations to little curvatures introduced in the taperwaist interfaces during stretching that radiate part of the energy, and slight changes in the transition region that modify the coupling between the fundamental mode of the fiber and modes excited in the waist.

Both tapered fibers were set in line by direct connection of their untapered extremes and its combined response measured for three different elongations of the waist of the second taper, as shown in Fig. 3. The resulting response resembles that of a single taper with increased visibility and modulated in amplitude. The theoretical periodicity of this modulation is $\mathrm{FSR}_{1} \cdot \mathrm{FSR}_{2} /\left(\mathrm{FSR}_{1}-\mathrm{FSR}_{2}\right)$, being $\mathrm{FSR}_{i}$ the $i$ th taper response.

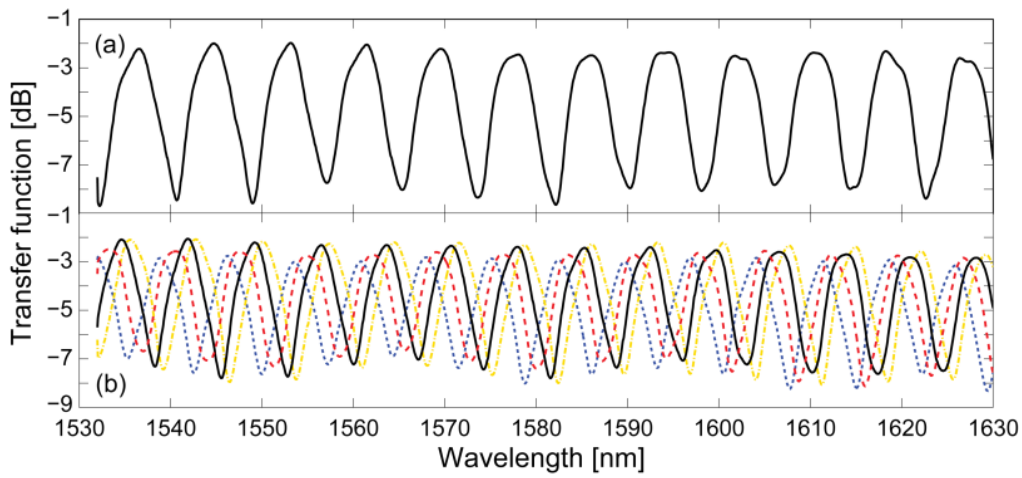

Fig. 2. (a) Experimental transfer functions of the fixed and (b) stretched biconical tapers. 
Experiments fit the predicted value of $57 \mathrm{~nm}$. Since the combined response matches the product of the individual ones, it can be concluded that additional interference phenomena, such as reflections between the tapered fibers, do not contribute significantly to the total response. Also, because the phase shift introduced in the stretched taper shifts the combined response, small phase changes originate large frequency shifts for the envelope. This may be useful in the development of fiber-based sensors with increased sensitivity. In the present work, this technique is used to shape femtosecond optical pulses dynamically.

Figure 4 shows the experimental setup and preliminary measurements. A fiber laser generates 100 -fs pulses at a repetition rate of $50 \mathrm{MHz}$ with an average power of $7 \mathrm{dBm}$. A section of dispersion compensating fiber (DCF) with $-0.67 \mathrm{ps} / \mathrm{nm}$ of accumulated dispersion is employed before the pulse-shaping stage (PSS) to avoid nonlinear effects in the tapers arising from pulses with high peak power and to precompensate for the dispersion suffered by the pulses along the standard single-mode fiber (SSMF) placed at the output of the experimental setup. A 99/1 power splitter is set at the output of the tapers to observe the spectrum and autocorrelation function of the processed pulses by means of an optical spectrum analyzer (OSA) and an intensity optical autocorrelator $(\mathrm{OA})$, based on harmonic generation in a nonlinear crystal, respectively. A high-power booster benchtop Erbium-doped fiber amplifier (EDFA), from Keopsys, was included before the SSMF to boost the signal. It provided amplification with a saturated output of $18 \mathrm{dBm}$ and return loss below $-40 \mathrm{~dB}$ over the whole C-band so as not to filter the spectrum of the signals to be amplified. Although stretching and compression of the pulses was performed to amplify them up to the

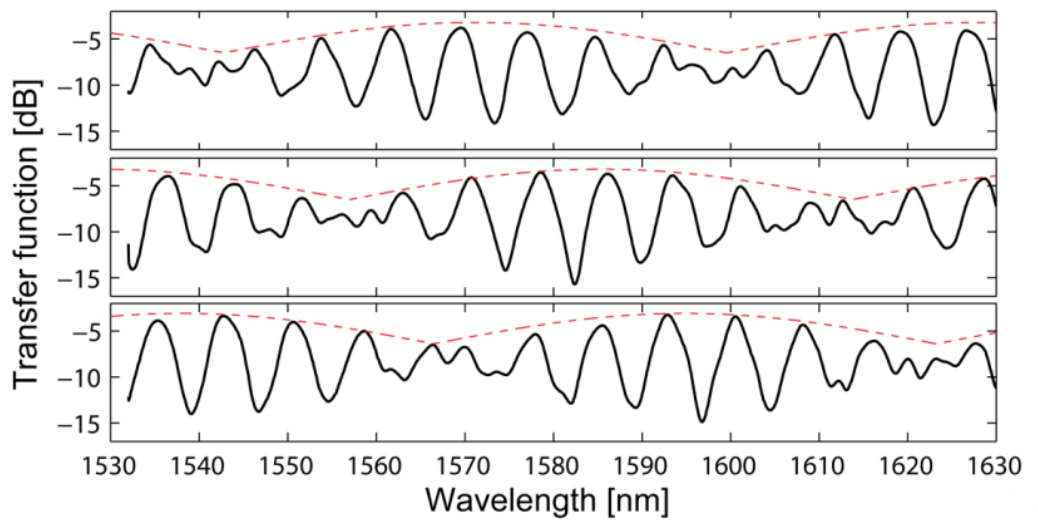

Fig. 3. Transmission response of the inline combination of fixed and stretched tapered fibers for different elongation. 

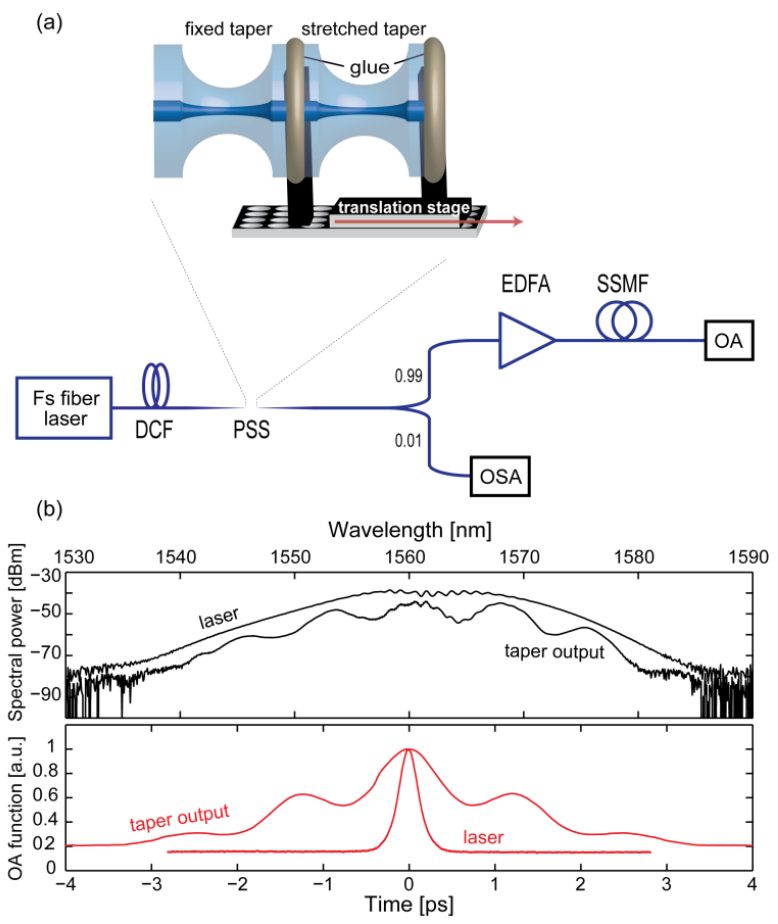

Fig. 4. (a) Experimental setup and (b) experimental measurements illustrating the concept of pulse shaping through taper cascading.

sensitivity level of the OA without exciting nonlinear effects in the tapers, the processing technique does not rely on dispersion and can be implemented using only the few $\mathrm{cm}$ of fibers that are necessary to fabricate the tapered fibers.

The spectrum and autocorrelation functions of both the laser output and shaped pulses are shown in Fig. 4(b). In this case, the response of the tunable taper was shifted to overlap the response of the fixed taper in the bandwidth of the femtosecond laser. In-phase interference (i.e., the elongation point where the spectral responses of the tapered fibers interfere with similar phases for the spectrum of the laser), at these wavelengths results in fringes with increased visibility. Without tapers, the optical autocorrelation resolves pulses with a full width at halfmaximum (FWHM) duration of 200 fs. Since these recompressed pulses are close to their transform-limited duration, all previous dispersion is nearly completely compensated. Consequently, when the cascaded tapers are included in the setup, the optical pulses measured at the OA are widened due to the dispersion induced by the tapered fibers themselves. 


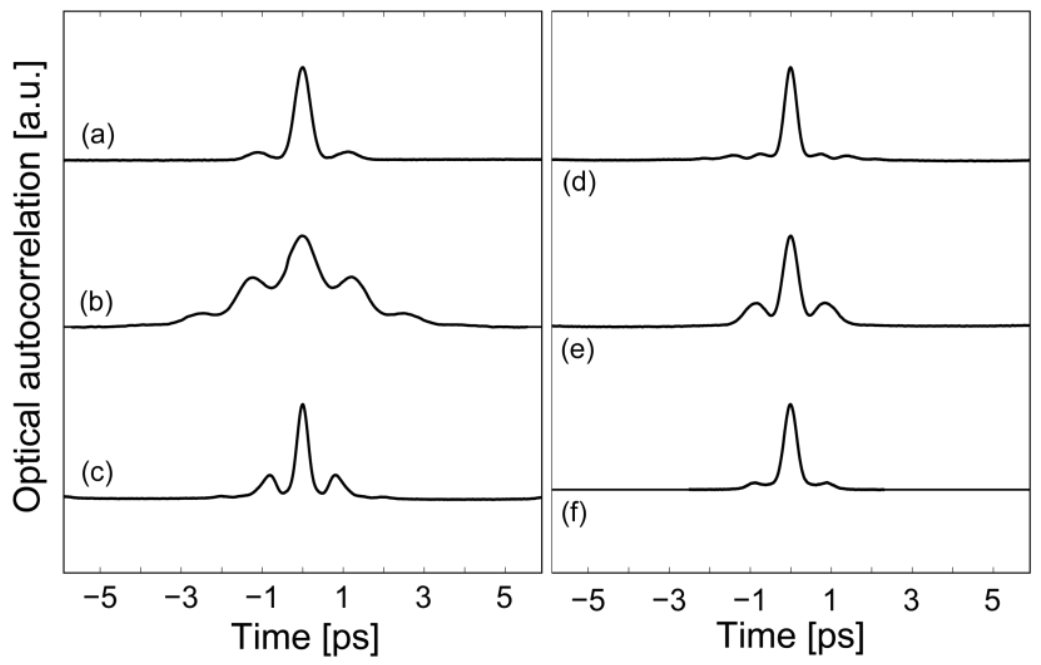

Fig. 5. Optical autocorrelation measurements with dispersion compensation of (a) one taper fiber, (b) in-phase interference of both tapers, (c)-(f) cascaded combinations for different interference points.

Figure 5 shows optical autocorrelation traces for the inline combination of two tapered fibers after the compensation of the dispersion present in the setup, with the exception of Fig. 5(a) that shows the OA function of only the tunable taper for comparison purposes. Figure 5(b) corresponds with the cascaded combination of the two tapered fibers under approximate in-phase interference in the laser bandwidth. Figures 5(c)-5(f) show several pulse train profiles obtained for different interference points. These results show that the number of pulses as well as their amplitude distribution can be controlled by mechanical stretching of the tunable taper. Moreover, when the amount of uncompensated dispersion in the setup widens, the pulses, up to a duration that exceeds the delay introduced by the modal dispersion experienced by the modes propagating in the waist the pulses, overlap in time and interfere and a train of pulses with a repetition frequency that depends on the uncompensated dispersion is generated. For the femtosecond pulses considered here, an uncompensated dispersion of a few meters of SSMF is enough to generate such an interference pattern. This way, dispersion can be used to scale the resulting signal in the timedomain by inserting and removing pieces of SSMF to obtain uncompensated dispersion. Measurements shown in Fig. 6 are for a constant configuration of the fiber tapers point and steps of $10 \mathrm{~m}$ of SSMF. When dispersion is perfectly compensated, the pulse train 
resembles those of Fig. 5. In the rest of traces, the delay between pulses in the train is changed from less than 1 ps to more than 2 ps. Although using dispersion to change the repetition frequency of the generated train of pulses seems interesting, broadening and reduction of peak power may limit its applicability.

In conclusion, we have proposed a novel technique to implement dynamic spectral responses, based on cascading of nonadiabatic fiber tapers. These have been fabricated and their individual and combined responses measured. The transfer function of the whole structure has been controlled by mechanical stretching of a single taper. In this work, this technique has been applied to the shaping of ultrashort optical pulses, although several applications may benefit from this approach, such as stress and temperature sensing, with enhanced sensitivity. We obtained stable and repeatable measurements thanks to the robustness of the relatively large tapers employed. The approach is not only scalable and simple, since it requires only processed pieces of standard fiber, but also allows for signal processing in a bandwidth of several terahertz while introducing little losses and dispersion. Techniques using dispersion allow for quasi-arbitrary waveform generation although compensation of high order dispersion terms becomes difficult. Since our approach does not rely on dispersion, it should be useful in applications where the duration of the pulse is critical. For example, in the generation of terahertz radiation where trains of femtosecond pulses are employed

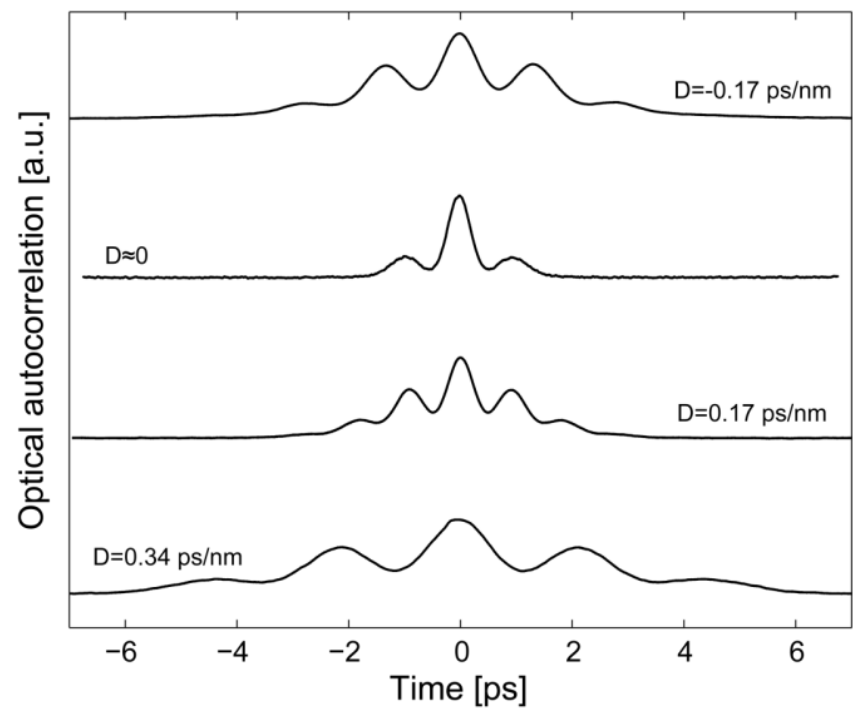

Fig. 6. Optical autocorrelation measurements of the two tapered fibers in line combination for different values of dispersion. 
to increase the spectral brightness of the emitter, or to provide controlled light-matter interaction in nonlinear optics. On the other side, the effect of uncompensated dispersion in the fiber link has been studied and proved useful to generate additional waveforms through interpulse interference. This increases the range of waveforms that can be generated at the expense of pulse widening. Alternative ways of changing the operating conditions of the tapers should be considered. For example, piezoelectric actuators and energetic optical fields capable of introducing nonlinear effects in the taper are expected to provide electrical and optical control, respectively.

\section{References}

[1] J.P. Goure, and I.Verrier, 'Optical Fibre Devices', CRC Press, 2010.

[2] G. P. Agrawal, 'Fiber-Optic Communication Systems', WileyInterscience, 2002.

[3] K. Jedrzejewski, 'Biconical fused taper - a universal fibre devices technology', Opto-Electronics Review 8, 2, 153-159, 2000.

[4] S. Lacroix, F. Gonthier, R. J. Black, and J. Bures, 'Tapered-fiber interferometric wavelength response: the achromatic fringe', Optics Letters 13, 5, 395-397, 1988.

[5] J. Villatoro, D. Monzón-Hernández, and E. Mejía, 'Fabrication and modeling of uniform-waist singlemode tapered optical fiber sensors', Applied Optics 42, 13, 2278-2283, 2003.

[6] D. Monzón-Hernández, and J. Villatoro, 'High-resolution refractive index sensing by means of a multiple-peak surface plasmon resonance optical fiber sensor', Sensors and Actuators B 115, 1, 227-231, 2006.

[7] J. Villatoro, D. Monzón-Hernández, and D. Luna-Moreno, 'In-line optical fiber sensors based on cladded multimode tapered fibers', Applied Optics 43, 32, 5933-5938, 2004.

[8] Z. Wang, H. Sone, Y. Tsuji, and M. Imai, 'Spectral Phase and Intensity Evolutions of Supercontinuum Generation in a Biconical Tapered Fiber', Optical Review 14, 2, 81-85, 2007. 
[9] F. Lu, and W. H. Knox, 'Generation of a broadband continuum with high spectral coherence in tapered single-mode optical fibers', Optics Express 12, 2, 347-353, 2004.

[10] K. Jedrzejewski, and A. Kosinski, 'Wide-band tapered directional coupler', Proceedings of the SPIE Conference 3189, 130-132, 1997.

[11] B. S. Kawasaki, K. O. Hill, and R. G. Lamont, 'Biconical-taper singlemode fiber coupler', Optics Letters 6, 7, 327-328, 1981.

[12] M. Rusu, R. Herda, S. Kivistö, and O. G. Okhotnikov, 'Fiber taper for dispersion management in a mode-locked ytterbium fiber laser', Optics Letters 31, 15, 2257-2259, 2006.

[13] K. Q. Kieu, and M. Mansuripur, 'Femtosecond laser pulse generation with a fiber taper embedded in carbon nanotube polymer composite', Optics Letters 32, 15, 2242-2244, 2007.

[14] A. M. Weiner, 'Ultrafast optical pulse shaping', Optics Communications 284, 15, 3669-3692, 2011.

[15] X. Liu, A. B. Premkumar, and A. S. Madhukumar, 'Pulse Shaping Functions for UWB Systems', IEEE Transactions on Wireless Communications 7, 5, 1512-1516, 2008.

[16] A. S. Weling, and T. F. Heinz, 'Enhancement in the spectral irradiance of photoconductiong terahertz emitters by chirped-pulse mixing', Journal of the Optical Society of America B 16, 9, 1455-1467, 1999.

[17] A. M. Weiner, J. P. Heritage, and E. M. Kirschner, 'High-resolution femtosecond pulse shaping', Journal of the Optical Society of America B 5, 8, 1563-1572, 1988.

[18] R. E. Saperstein, N. Alic, D. Panasenko, R. Rokitski, and Y. Fainman, 'Time-domain waveform processing by chromatic dispersion for temporal shaping of optical pulses', Journal of the Optical Society of America B 22, 11, 2427-2436, 2005.

[19] T. A. Birks, and Y. W. Li, 'The shape of fiber tapers', Journal of Lightwave Technology 10, 4, 432-438, 1992.

[20] K. Q. Kieu, and M. Mansuripur, 'Biconical Fiber Taper Sensors', IEEE Photonics Technology Letters 18, 21, 2239-2241, 2006. 
[21] G. Cohoon, C. Boyter, M. Errico, K. Vandervoort, and E. Salik, 'Enhancing sensitivity of biconical tapered fiber sensors with multiple passes through the taper', Optical Engineering 49, 3, 034401-034401-5, 2010.

[22] W. B. Ji, H. H. Liu, S. C. Tjin, K. K. Chow, and A. Lim, 'Ultrahigh Sensitivity Refractive Index Sensor Based on Optical Microfiber', IEEE Photonics Technology Letters 24, 20, 1872-1874, 2012. 


\section{Paper E}

\section{"Low-Cost Refractive Index and Strain Sensor Based on Tapered Fibers"}

S. Mas, J. Martí, D. Monzón-Hernández, and J. Palací

Submitted to Optics Communications 


\title{
Low-cost refractive index and strain sensor based on tapered fibers
}

\author{
S. Mas, ${ }^{1, *}{ }^{*}$. Martí, ${ }^{1}$ D. Monzón-Hernández, ${ }^{2}$ and J. Martín ${ }^{1}$ \\ ${ }^{1}$ Valencia Nanophotonics Technology Center, Universitat Politècnica de \\ València, 46022 Valencia, Spain \\ ${ }^{2}$ Centro de Investigaciones en Óptica A.C., 37150 Guanajuato, México
}

\begin{abstract}
In this paper, biconical tapered fibers are used to carry out sensing in a simple and effective way by using an incoherent source and a power meter. This approach reduces the cost and complexity of traditional schemes based on tunable lasers while providing similar performance. Refractive index and strain sensing measurements are presented and their performance discussed.
\end{abstract}

\section{Introduction}

Optical fiber is the preferred transmission medium for different applications due to its large bandwidth, immunity to electromagnetic interferences and little attenuation, among others [1]. Fiber geometry can be changed in several ways to modify its characteristics and fabricate different devices such as amplifiers, modulators, interferometers, directional couplers, filters, etc. [2]. Among these devices, biconical tapered fibers are fabricated through the application of heat and mechanical strain [3]. They consist of a uniform waist located between two transition regions. Depending on the length of the transition two types of tapers can be distinguished. When the transition is smooth or adiabatic the fundamental mode of the fiber propagates normally. However, if the transition is abrupt or non-adiabatic the fundamental fiber mode originates several modes. These modes have different effective indexes and therefore experience different 
propagation lengths. Once the modes are recoupled into the fundamental mode of the standard fiber the interference between these modes creates a spectral pattern that depends on the difference between their effective refractive indexes and the waist length, resembling the spectrum of a Mach-Zehnder interferometer (MZI) when a few modes gather most of the total power [4].

Sensing is of widespread use in several fields such as medicine, communications and imaging systems, among others [5]. Two important types of sensing that rely on optical technology are strain [6] and refractive index (RI) [7] sensors. The quality of a strain or refractive index sensor is quantified through the sensor sensitivity [8], whose expression relates the shift induced in the sensor response per unit of change. Strain sensing has been carried out using different devices such as Bragg gratings [9], photonic crystal fibers [10] or ZnO piezoelectric wires [11]. On the other side, different structures and technologies have been used to sense RI [7], like surface plasmon resonance (SPR) [12], ring resonators [13] or photonic crystals [14], among others.

Non-adiabatic biconical tapered fibers are used for both RI and strain sensing [15-17]. RI and strain sensors consist of a continuous wave (CW) laser, the sensing structure and a power meter [18-20]. Although they provide good sensitivity by tuning the pump wavelength into the maximum slope point of the tapered fiber's response, CW lasers are costly and experience long-term wavelength and power drifts that must be compensated using a wavelength-meter together with a digitalanalog feedback system [21]. To alleviate this problem, a viable solution is the use of an incoherent broadband source (e. g. a diode). In this case the power detected corresponds not with individual points of the transfer function but with the integration of a wavelength span. The sensitivity is initially reduced because of this power integration, although the main noise source (laser's amplitude and wavelength changes) disappears so electrical post-amplification can be used to obtain similar sensitivities. Therefore in the latter approach source cost and complexity are considerably reduced. Although the use of a broadband source can lead to lower extinction ratios they can be increased by introducing a combination of tapered fibers so that the performance provided by the two approaches is comparable.

In this paper, the characterization of refractive index and strain sensors based on the inline combination of biconical taper fibers and use of an incoherent broadband source is presented and compared with the results obtained by the standard approach. The comparison between the two approaches exposed here is very useful when designing sensing measurements based on fibers as it takes into account critical aspects 
such as the experimental setup cost, the sensor sensitivity and the source stability. The use of biconical tapered fibers reduces dramatically the cost of the system because of the simplicity and low cost of its fabrication process. In addition, the cascaded combination of tapered fibers provides flexibility and versatility to the sensing scheme. Moreover, an increase in the sensitivity of the sensor by using the two tapered fibers combination is demonstrated for the first time to the author's knowledge. Finally, the use of an incoherent source lowers the cost of the system and provides increased amplitude stability that compensates for lower dynamic ranges. Therefore, the proposed setup reduces the cost of the system while increasing the sensor sensitivity, flexibility and amplitude stability.

\section{Principle of operation}

Figure 1 shows the two different methods considered to carry out sensing measurements. Spectral variations in the response of the device can be detected by using a tunable laser emitting in the maximum slope point of the response, as depicted in Fig. 1(a). The shift direction can be determined by monitoring variations of the output power level. This method leads to a good extinction ratio and sensitivity, but also to higher costs and complexity. Similar results can be obtained by the cascaded combination of tapered fibers using a broadband source, as shown in Fig.

(a)
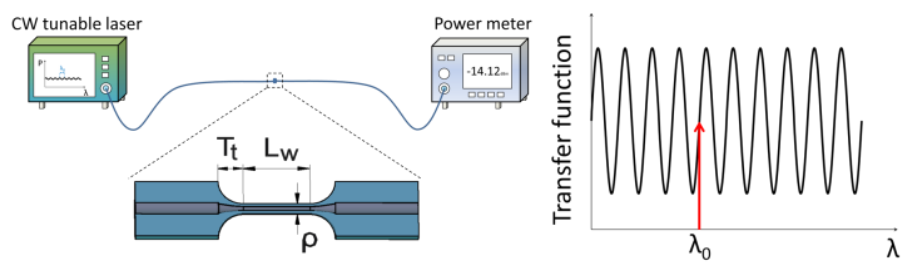

(b)
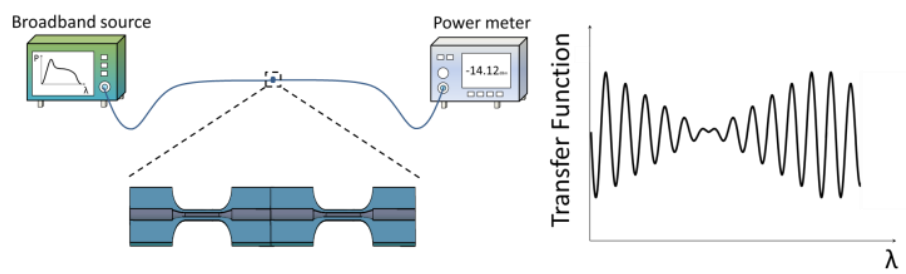

Fig.1. Sensor structures when considering (a) a CW tunable laser with a single tapered fiber and (b) an incoherent broadband source with the inline combination of two tapered fibers. 
1(b), resulting in a simpler and cheaper experimental setup. This approach takes advantage of the increased extinction ratio obtained in the in-phase interference region of the response of the tapered fibers. In this paper, tapered fibers characterization was accomplished by means of a tunable laser, and sensing measurements were carried out using both a broadband source and a tunable laser for comparison purposes. A power meter was used in all cases to monitor the response.

Two biconical tapered fibers, Taper A and Taper B from now on, with geometrical parameters $\mathrm{T}_{\mathrm{t}}=1 \mathrm{~mm}, \mathrm{~L}_{\mathrm{w}}=13 \mathrm{~mm}$ and $\rho=18 \mu \mathrm{m}$ were fabricated, where $T_{t}$ is the coupling regions length and $\rho$ and $L_{w}$ are the waist diameter and length, respectively, as shown in Fig. 1(a). Optical characterization of the two tapered fibers and its inline combination is shown in Fig. 2. The free spectral range (FSR) values of the tapered fibers are $\mathrm{FSR}_{A}=8 \mathrm{~nm}$ and $\mathrm{FSR}_{\mathrm{B}}=8.2 \mathrm{~nm}$, respectively. Differences are originated from small deviations in the fabrication process. The resulting FSR from the cascaded combination of the two tapered fibers is given by $\left(\mathrm{FSR}_{1} \cdot \mathrm{FSR}_{2}\right) /\left(\mathrm{FSR}_{1}-\mathrm{FSR}_{2}\right)$ [4], which in this case is $328 \mathrm{~nm}$. The resulting FSR is higher than the sweep bandwidth of $100 \mathrm{~nm}$, and therefore a complete period is not shown in Fig. 2(c). Taper A was stretched longitudinally after fixing its two ends onto a translation stage by means of two drops of glue in contact with the cladding of the untapered part of the fiber. Both tapered fibers were set in line by direct connection of

(a)

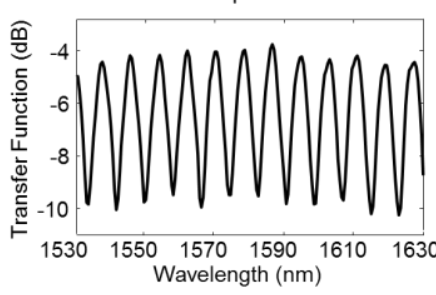

(b)

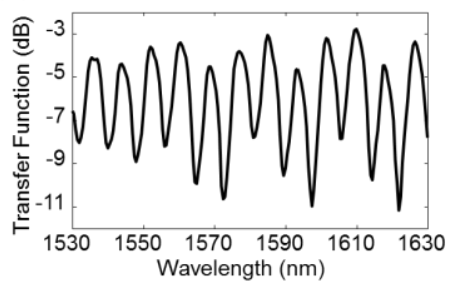

(c)

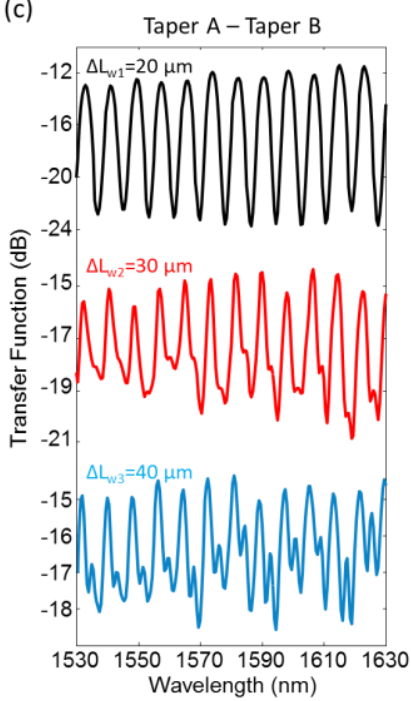

Fig.2. Strain optical characterization by means of a tunable laser and a power meter of (a) isolated Taper A, (b) isolated Taper B and (c) their inline combination for three different points of elongation of Taper $\mathrm{A}, \Delta \mathrm{Lw}_{1}, \Delta \mathrm{Lw}_{2}$ and $\Delta \mathrm{Lw}_{3}$ 
their untapered extremes and the resulting transfer function for the combination of Taper A-Taper B when considering three different elongation points of Taper $\mathrm{A}, \Delta \mathrm{L}_{\mathrm{w} 1}=20 \mu \mathrm{m}, \Delta \mathrm{L}_{\mathrm{w} 2}=30 \mu \mathrm{m}$ and $\Delta \mathrm{L}_{\mathrm{w} 3}=40 \mu \mathrm{m}$, is shown in Fig. 2(c). As can be seen, depending on the constructive interference provided by the sum of the individual responses of the two tapered fibers while stretching Taper A, different maximum extinction ratios are obtained, varying from $3 \mathrm{~dB}$ to $12 \mathrm{~dB}$.

Considering that the fundamental mode power mainly splits into two modes, the operating principle of the tapered fiber sensors is based on the transfer function dependence on the waist length and the index difference between the two nearest cladding modes as follows [4]:

$$
T F \sim \sin \left(k_{0} L_{W}\left(n_{e f f 1}-n_{e f f 2}\right)+\varphi_{0}\right)
$$

where $\mathrm{k}_{0}$ is the angular wavenumber in vaccum, $\varphi_{0}$ is the phase offset and $n_{\text {eff } 1}$ and $n_{\text {eff2 }}$ are the effective indexes of the fundamental cladding mode and the first order cladding mode, respectively. This leads to strain and refractive index sensing in a wide bandwidth thanks to a single structure. System calibration was realized for refractive index variations as well. Four different outer mediums were considered, i.e. deionized water (DIW) in addition to $2 \%, 4 \%$ and $6 \%$ Ethanol concentration in DIW, with refractive indexes at $1550 \mathrm{~nm}$ and ambient temperature of $\mathrm{n}_{\text {DIW }}=1.3173, \mathrm{n}_{2 \% \mathrm{Eth}}=1.3186, \mathrm{n}_{4 \% \mathrm{Eth}}=1.3199$ and $\mathrm{n}_{6 \% \mathrm{Eth}}=1.3212$, respectively [22]. Figure 3 shows the transference function of Taper A and the inline combination Taper A-Taper B for these four different surrounding mediums. Taper A maximum extinction ratio decreases because of the increased evanescence of the optical field for surrounding mediums with higher refractive index, varying from $6 \mathrm{~dB}$ in air to $5 \mathrm{~dB}$ in DIW. Besides, it considerably falls when the refractive index is even higher, reaching maximum extinction ratios of $3 \mathrm{~dB}$, as shown in Fig. $3(\mathrm{a})$. This is due to the different absorption coefficient of the ethanol. On the other side, the maximum extinction ratio of the cascaded combination of the two tapered fibers rises slightly when increasing the refractive index from air to DIW due to a phase shift in the total transfer function. This provided a higher maximum extinction ratio in the optical response, as depicted in Fig. 3(b). When considering higher refractive indexes through different ethanol concentrations in DIW, the inline combination transference function presents lower maximum extinction ratio because the phase shift leads to a point of destructive interference between the individual transfer functions. 
(a)

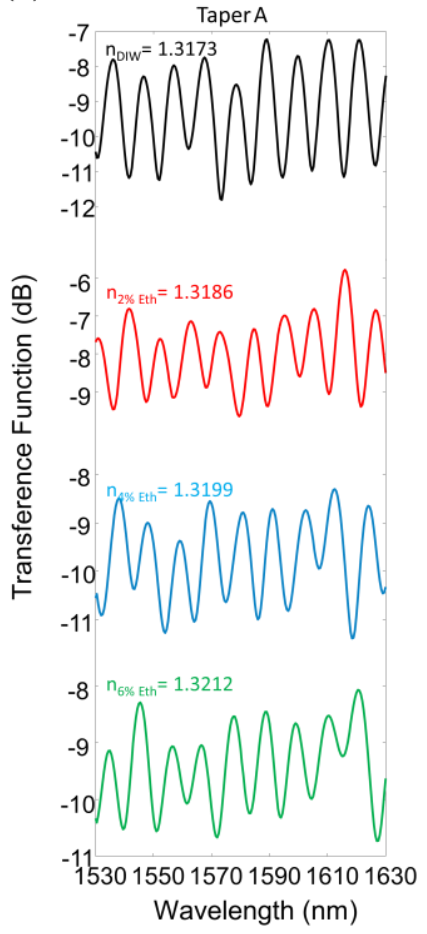

(b)

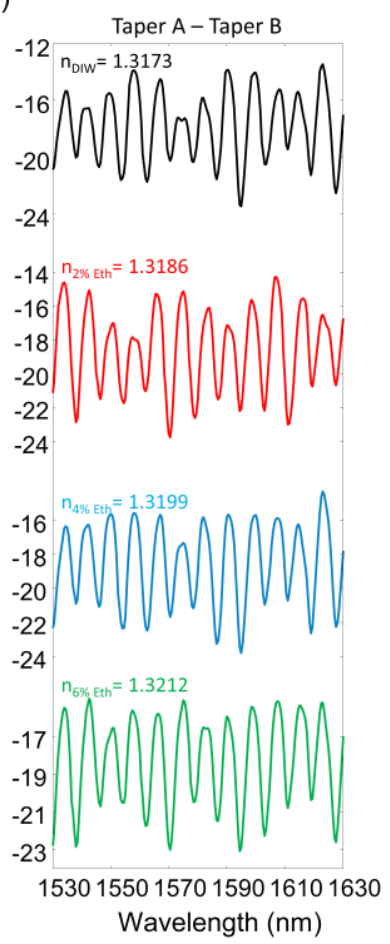

Fig.3. RI sensing optical characterization by means of a tunable laser and a power meter of (a) Taper A, and the cascaded combination of (b) Taper A-Taper B when considering DIW, $\%, 4 \%$ and $6 \%$ ethanol concentration in DIW (from top to bottom plots).

\section{Experimental Results and Discussion}

Strain sensing was performed using both a tunable laser and a broadband source for comparison purposes. A power meter was employed to monitor the results instead of an optical spectrum analyzer in order to reduce costs in the experimental setup. Therefore, the sensitivity value is expressed in terms of extinction ratio as $\mathrm{S}_{\Delta \mathrm{L}}=\Delta \mathrm{P} / \Delta \mathrm{L}_{\mathrm{w}}$. Figure 4(a) shows the transference function obtained when considering a tunable laser source while Taper A is stretched. When the stretching increases, the tapered fiber is elongated and the optical response varies. This approach exhibits a maximum extinction ratio of $6.3 \mathrm{~dB}$, thus the sensitivity value obtained for Taper $A$ is $S_{\text {tunable } A}=0.54 \mathrm{~dB} / \mu \mathrm{m}$. On the other side, when a broadband source is used, Fig. 4(b), the maximum 

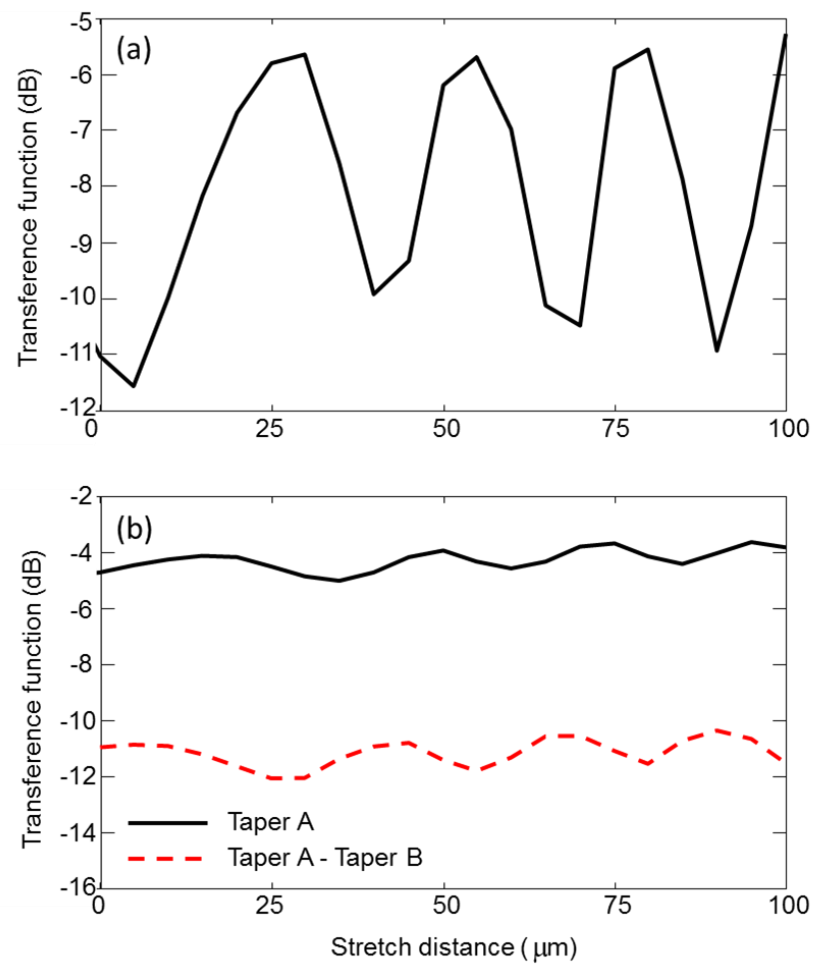

Fig.4. Strain sensing measurements by means of a power meter and a (a) tunable laser and (b) broadband source.

extinction ratio decreases providing sensitivity values for Taper A and the inline combination of the tapered fibers Taper A-Taper B of

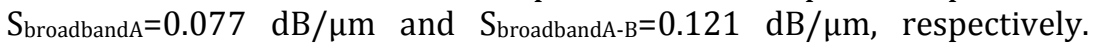
Sensitivity value obtained of the inline combination is higher than that of the isolated Taper A because of the enhancement in extinction ratio achieved by the cascaded combination. Therefore, using a broadband source instead of a tunable laser is a very useful approach as it presents good values of sensitivity in addition to lower system costs and higher stability.

Refractive index sensing measurements were also realized by means of a broadband source. Taper A was used for sensing the outer mediums previously mentioned, i.e. DIW and 2\%, 4\% and 6\% ethanol concentration in DIW, in addition to air ( $\left.n_{\text {air }}=1\right)$, as depicted in Figure 5. Considering the RI sensor sensitivity given by $S_{R I}=\Delta P / \Delta n$, the sensitivity values obtained for Taper A and the inline combination Taper A-Taper B are $S_{\text {broadbandA }}=907.69 \mathrm{~dB} / \mathrm{RIU}$ and $S_{\text {broadbandA-B }}=2261.53 \mathrm{~dB} / \mathrm{RIU}$, 
respectively. It can be seen how the combination of two tapered fibers increases considerably the sensitivity.

Power stability for the two approaches considered was characterized at the same temperature conditions and for the same biconical tapered fiber using a power meter. An EXFO FLS-2600 Laser Source was used in both modes, tunable and ASE. As can be seen in the normalized transfer function shown in Figure 6, the broadband source provides higher stability than the tunable source with a deviation of $0.039 \mathrm{~dB}$ in comparison with the peak to peak variation of $0.139 \mathrm{~dB}$ of the tunable laser approach.

\section{Conclusion}

In conclusion, we have proposed and demonstrated refractive index and strain sensing in a simple way just by means of biconical tapered fibers in a broad bandwidth. This was carried out using a low-cost experimental setup consisting on a broadband source and a power meter. The cascaded combination of two tapered fibers allowed enhancing the extinction ratio of the resulting transference function to increase the performance of the system. In addition, results obtained using a tunable laser are shown for comparison purposes. RI and strain sensing measurements as well as power stability evolution in both approaches are presented and analyzed. This work shows the versatility and great applicability of biconical tapered fibers in sensing systems by means of the proposed stable and low-cost experimental setup.

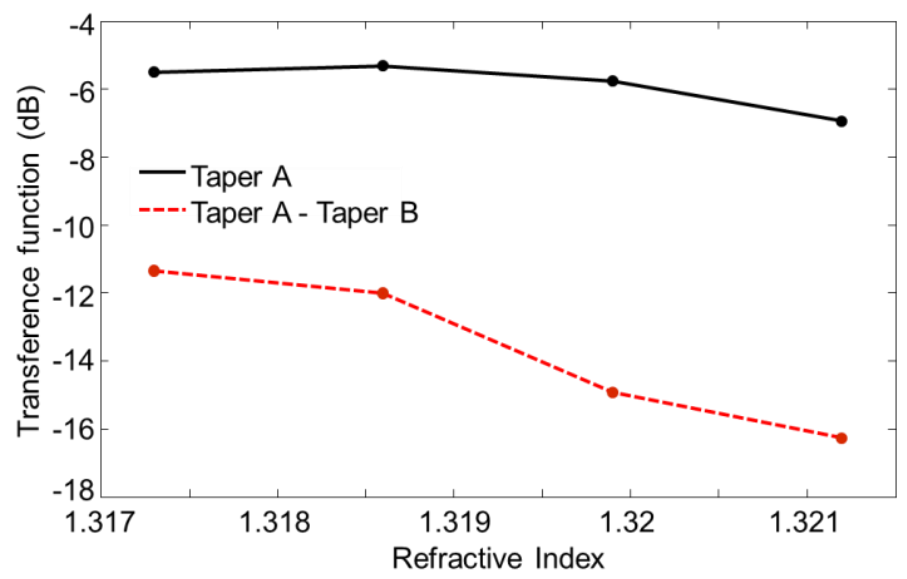

Fig.5. RI sensing measurements by means of a broadband source and a power meter. 


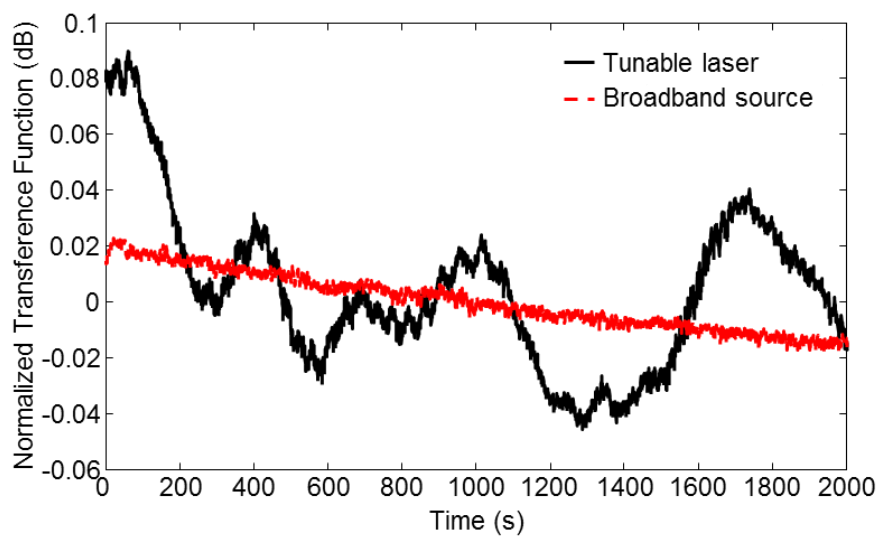

Fig.6. Power stability of a single tapered fiber measured by means of a tunable laser and a broadband source.

\section{References}

[1] G. P. Agrawal, 'Fiber-Optic Communication Systems', WileyInterscience, 2002.

[2] J.P. Goure, and I. Verrier, 'Optical fibre devices', CRC Press, 2002.

[3] J. Villatoro, D. Monzón-Hernández, and E. Mejía, 'Fabrication and Modeling of Uniform-Waist Single-Mode Tapered Optical Fiber Sensors', Applied Optics 42, 13, 2278-2283, 2003.[4] S. Mas, J. Palací, P. Pérez-Millán, S. Lechago, D. Monzón-Hernández, and J. Martí, 'All-fiber processing of terahertz-bandwidth signals based on cascaded tapered fibers', Optics Letters 37, 23, 4954-4957, 2013.

[4] S. Mas, J. Palací, P. Pérez-Millán, S. Lechago, D. Monzón-Hernández, and J. Martí, 'All-fiber processing of terahertz-bandwidth signals based on cascaded tapered fibers', Optics Letters 38, 23, 4954-4957, 2013.

[5] D. Ahuja, and D. Parande, 'Optical sensors and their applications', International Journal of Scientific Research and Reviews 1, 5, 60-68, 2012.

[6] G.B. Hocker, 'Fiber-optic sensing of pressure and temperature', Applied Optics 18, 9, 1445-1448, 1979.

[7] I.M. White, and X. Fan, 'On the performance quantification of resonant refractive index sensors', Optics Express 16, 2, 1020-1028, 2008. 
[8] X. Fan, I.M. White, S.I. Shopova, H. Zhu, J.D. Suter, and Y. Sun, 'Sensitive optical biosensors for unlabeled targets: A review', Analytica Chimica Acta 620, 8-26, 2008.

[9] A.D. Kersey, T.A. Berkoff, and W.W. Morey, 'Multiplexed fiber Bragg grating strain-sensor with a fiber Fabry-Perot wavelength filter', Optics Letters 18, 16, 1370-1372, 1993.

[10] X. Dong, H.Y. Tam, and P. Shum, 'Temperature-insensitive strain sensor with polarization-maintaining photonic crystal fiber based Sagnac interferometer', Applies Physics Letters 90, 151113, 2007.

[11] J. Zhou, Y. Gu, P. Fei, W. Mai, Y. Gao, R. Yang, G. Bao, and Z.L. Wang, 'Flexible Piezotronic Strain Sensor', Nano Letters 8, 9, 3035-3040, 2008.

[12] J. Homola, S.S. Yee, and G. Gauglitz, 'Surface plasmon resonance: review', Sensors and Actuators B-Chemical 54, 3-15, 1999.

[13] C.A. Barrios, K.B. Gylfason, B. Sánchez, A. Griol, H. Sohlström, M. Holgado, and R. Casquel, 'Slot-waveguide biochemical sensor', Optics Letters 32, 21, 3080-3082, 2007.

[14] J. Jágerská, H. Zhang, Z. Diao, N. Le Thomas, and R. Houdré, 'Refractive index sensing with an air-slot photonic crystal nanocavity', Optics Letters 35, 15, 2523-2525, 2010.

[15] K.Q. Kieu, and M. Mansuripur, 'Biconical fiber taper sensors', IEEE Photonics Techonology Letters 18, 21, 2239-2241, 2006.

[16] W.B. Ji, Y.C. Tan, B. Lin, S.C. Tjin, and K.K. Chow, 'Nonadiabatically tapered microfiber sensor with ultrashort waist', IEEE Photonics Technology Letters 26, 22,2303-2306, 2014.

[17] F.J. Arregui, I.R. Matías, and M. López-Amo, 'Optical fiber gauge based on a tapered single-mode fiber', Sensors and Actuators A 79, 2, 90-96, 2000.

[18] A. Asseh, S. Sandgren, H. Ahlfeldt, B. Sahlgren, R. Stubbe, and G. Edwall, 'Evanescent field refractive index sensor utilizing a narrow fibre Bragg grating and a tunable DBR laser', Proceedings of the SPIE Conference 2836, 57, 1996.

[19] P.S. Nunes, N.A. Mortensen, J.P. Kutter, and K.B. Morgensen, 'Refractive index sensor based on 1D photonic crystal in a microfluidic channel', Sensors 10, 2348-2358, 2010.

[20] K. De Vos, I. Bartolozzi, E. Schacht, P. Bienstman, and R. Baets, 'Silicon-on-insulator microring resonator for sensitive and labelfree biosensing', Optics Express 15, 12, 7610-7615, 2007. 
[21] S. Kobtsev, S. Kandrushin, and A. Potekhin, "Long-term frequency stabilization of a continuous-wave tunable laser with the help of a precision wavelengthmeter', Applied Optics 46, 23, 5840-5843, 2007.

[22] J. García-Rupérez, V. Toccafondo, M.J. Bañuls, J. García Castelló, A. Griol, S. Peransi-Llopis, and A. Maquieira, "Label-free antibody detection using band edge fringes in SOI planar photonic crystal waveguides in the slow-light regime", Optics Express 18, 23, 2427624286, 2010. 


\title{
Paper F
}

\section{“Biconical Tapered Fibers Manipulation for Refractive Index and Strain Sensing Applications"}

\author{
S. Mas, J. Martí, and J. Palací
}

IEEE Sensors Journal, Volume 15, Number 3, pp. 1331-1335, 2015. 


\title{
Biconical tapered fibers manipulation for refractive index and strain sensing applications
}

\author{
S. Mas, J. Martí, and J. Palací \\ Valencia Nanophotonics Technology Center, Universidad Politécnica de \\ Valencia
}

\begin{abstract}
Tapered fibers are used in multitude of applications due to its great versatility and functionality. In particular, this type of fibers is especially useful for sensing applications. For this purpose, tapered fibers must be adequately manipulated to avoid disturbances in the sensor's response. In this paper, a guideline for the correct handling of biconical tapered fibers during refractive index and strain measurements is presented and analyzed considering several manipulation scenarios and its corresponding influence in the performance of the system.
\end{abstract}

\section{Introduction}

Biconical tapered fibers are devices designed to modify the propagation characteristics of optical fiber in a simple way [1]-[3]. They consist of a narrow waist between two tapered regions. Its fabrication process involves stretching the fiber while heating it. Thus, by controlling the stretching speed along with the heat intensity, different waist diameters and tapered regions length can be obtained [4]. This leads to two different field behaviors inside the tapered regions. On one hand, when the transition is gradual the fundamental mode propagates without experiencing any variation. However, if the transition is abrupt the fundamental mode suffers from higher order couplings. Tapered fibers have been used in many applications such as supercontinuum generation [5], coupling [6] or pulse shaping [7] among others. In addition, the use of tapered fibers in sensing applications [8]-[10] has been widely discussed. Nevertheless, the use of these fibers requires certain handling precautions in order to avoid variations in the transfer function that are not due to the sensing process itself. 
This paper provides several manipulation rules to consider when refractive index (RI) and strain sensing measurements are carried out using biconical tapered fibers. Different handling situations and its consequences in the sensor's response are considered and analyzed. Although the study included in this work uses tapered fibers with determined dimensions, the special attention in tapered fiber fragility for small waist diameters. Also, the fiber waist diameter has an important influence on the number of modes excited in the tapered region. Waist diameters smaller than one micrometer do not originate an interference pattern at $1.55 \mu \mathrm{m}$ because the $L P_{0 m}^{\text {cladding }}$ mode does not propagate through the waist while diameters larger than those reported in this work lead to more excited modes thus complicating the monitoring of the sensed parameter due to the complex spectral response of the device [11].

\section{Apparatus description}

The nonadiabatic tapered fiber used in the experiments is shown in Fig. 1 , including its main geometric parameters $\mathrm{T}_{\mathrm{t}}=1 \mathrm{~mm}, \mathrm{~L}_{\mathrm{w}}=13 \mathrm{~mm}$ and $\rho$ $=18 \mu \mathrm{m}$. When the fundamental mode propagates through the input taper it experiences higher order modes coupling and a power transference occurs between the core fundamental mode $L P_{01}^{\text {core }}$ to the two nearest cladding modes, the fundamental mode $L P_{01}^{\text {cladding }}$ and a higher order mode $L P_{0 m}^{\text {cladding }}$ [12]. These modes propagate though the waist with different effective indexes and interfere between them because of their different propagation constant. Part of the light which is not confined in the taper propagates evanescently around the waist. Simulations carried out by means of commercial software provided the effective indexes of the two modes propagating through the waist, 1.4444 and 1.4214 , respectively, by considering standard refractive index values for core and cladding at $1.55 \mu \mathrm{m}$. Once these modes reach

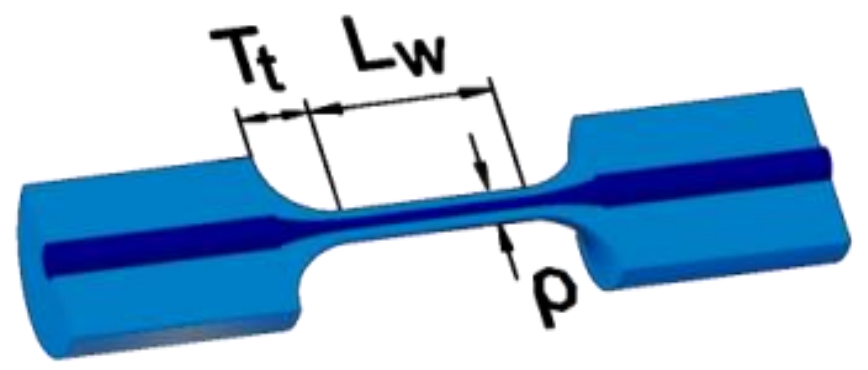

Fig. 1. Biconical tapered fiber section profile. 
the output tapered region they are re-coupled into the fundamental mode, leading to the creation of a spectral pattern which depends on the difference between the modes effective indexes and the waist length [7]. The resulting phase difference between the modes can be expressed as follows [12]:

$$
\Delta \phi=\frac{2 \pi \Delta n_{e f f} L}{\lambda}
$$

where $\Delta \mathrm{n}_{\text {eff }}$ is the effective refractive indexes difference between the two propagating modes, $\mathrm{L}$ is the interference length and $\lambda$ is the operating wavelength. When introducing a curvature in an optical fiber, its refractive index depends on the bending radius following the expression [13]:

$$
n=n_{0}\left[1+(1+\chi) \frac{x}{r}\right]
$$

where $\mathrm{n}_{0}$ is the refractive index in the corresponding non-curved optical fiber, $\mathrm{r}$ is the radius of the curvature, $\chi$ refers to the elasto-optic effect and $\mathrm{x}$ is the transverse coordinate of the bend. If the refractive index in the curvature is considerably higher than that of the outer medium, the mode experiences a low loss curve. Conversely, when the curvature refractive index is similar to the refractive index of the surrounding medium, the mode is poorly confined and suffers high radiation losses.

To sum up, variations in the waist length or effective indexes of the optical modes shift the interference pattern of the device while the introduction of curvatures has an effect on the degree of confinement experienced by the propagating fields. These effects can be monitored to build strain, refractive index and curvature sensors.

\section{Measurements procedure}

Sensing measurements were carried out using a tunable laser and a power meter. Refractive index sensing analysis takes into consideration the modification of the surrounding refractive index due to the placement of the tapered fiber, the position of the tapered fiber anchor points and the creation of bubbles or flows in the testing container. Strain sensing considers variations in the sensor response introduced by the improper bending of the tapered fiber.

Sensor performance parameters are obtained for both RI and strain measurements. Sensitivity is defined as the spectral shift experienced in the sensor response divided by unit of change. Therefore, when considering refractive index and strain sensing, sensitivity can be expressed as $\mathrm{S}_{\mathrm{RI}}=\Delta \lambda / \Delta \mathrm{n}[\mathrm{nm} / \mathrm{RIU}]$ and $\mathrm{S}_{\text {STRAIN }}=\Delta \lambda / \Delta \mathrm{Lw}[\mathrm{nm} / \mu \mathrm{m}]$, 
respectively. Moreover, the sensor resolution $\mathrm{R}$ is defined as the smallest variation that can be accurately detected. Furthermore, the detection limit of the sensor can be easily calculated as DL = R/S [14].

\section{A. Refractive index sensing}

As mentioned before, the fundamental mode propagating through the input tapered region experiences higher order modes coupling. Considering that the fiber mode is mainly divided into the mode traveling along the waist and the mode propagating in the fiber interface with the outer medium, the tapered fiber transfer function depends on the difference between these two modes effective indexes. Therefore, it is vital to pay attention to different situations that can alter the effective indexes leading to an erroneous sensing measurement. The first situation considered here is the tapered fiber placement. To avoid altering the outer refractive index, the tapered fiber must be in contact only with the medium to be sensed. To do this, one possible solution is to glue the tapered fiber into a structure that suspends it into the air. An example is shown in Fig. 2(a), where the tapered fiber is set on an optical delay line (ODL). Thus, the tapered fiber is fully surrounded by the external medium, air, providing true and reliable measurements. On the other side, when the tapered fiber is in contact with a surface, as seen in Fig. 2(b), the surface modifies the effective index value of the mode traveling through the cladding-outer medium erroneously modifying the sensor response.

The tapered fiber's transfer function measured under both scenarios, over surface and on surface, is shown in Fig. 3. The device was put inside of a recipient and three outer mediums were considered to carry out RI sensing measurements: air, deionized water (DIW) and 4\% ethanol concentration in DIW, with refractive indexes $\mathrm{n}_{\text {air }}=1$, nDIw $=$ 1.3173 and $n_{4 \% \text { Eth }}=1.3199$, respectively, at a wavelength of $1.55 \mu \mathrm{m}$ [15]. As can be seen in Fig. 3(a), when considering air as the outer medium the fringe pattern presents sinusoidal shape with a free spectral range (FSR) value of FSR air-over surface $=8.2 \mathrm{~nm}$ and a visibility of approximately $6.5 \mathrm{~dB}$. When considering higher refractive indexes as DIW, the extinction ratio decreases to $4.55 \mathrm{~dB}$ because of an increased evanescence of the optical field. In addition, the FSR value increases to FSRdiw-over surface $=10.5 \mathrm{~nm}$. Then, for an outer refractive index even higher, as that of ethanol, the FSR becomes $10.75 \mathrm{~nm}$ and the extinction ratio decreases to $3 \mathrm{~dB}$ because of the different absorption coefficient of ethanol. In these two cases, the sinusoidal shape of the fringe pattern remains. This sensor presents sensitivity and resolution values of $S_{R I}=1250 \mathrm{~nm} / \mathrm{RIU}$ and $\mathrm{R}=$ $8 \cdot 10^{-6}$, respectively, obtained assuming a resolution of $0.01 \mathrm{~nm}$ in the spectrum analyzer. These results are competitively compared with other refractive index sensors based on tapered fibers as those present in [16] 

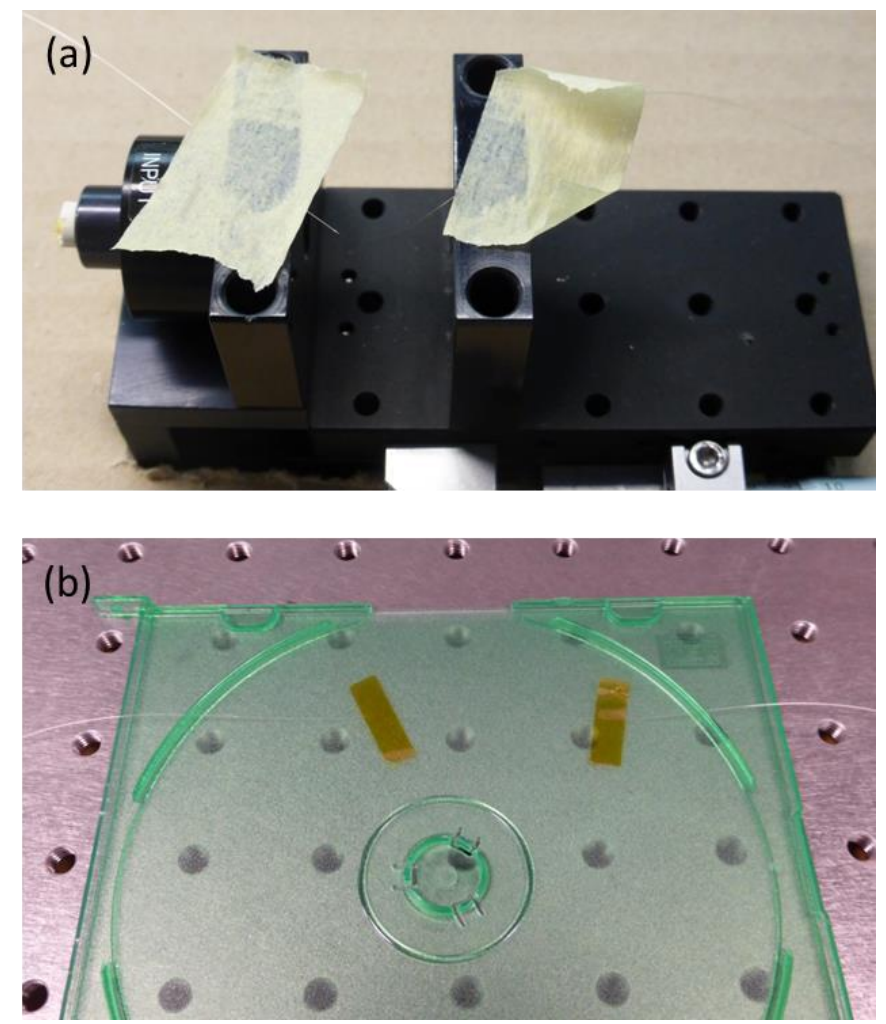

Fig. 2. Examples of biconical tapered fibers placed (a) over-surface and (b) on-surface.

and [17], which reported resolution values are $5.8 \cdot 10^{-6}$ and $8.5 \cdot 10^{-5}$, respectively. When the tapered fiber is placed on a surface part of the mode propagating through the cladding-outer medium interface varies leading to changes in the sensor's transference function as shown in Fig. 3(b). It can be observed how the tapered fiber interaction with the support surface causes the transfer function to lose its sinusoidal shape. In addition, the free spectral range value for the air case is slightly modified in comparison with the over-surface situation, resulting in a FSR of $8.36 \mathrm{~nm}$. When considering larger refractive indexes in the tapered fiber on-surface placement, as DIW and $4 \%$ ethanol concentration in DIW, the transfer function still presents a nonsinusoidal shape. The results suggest that more than two modes are present in the waist region. Approximate FSR values for DIW and 4\% Ethanol are FSR diw-on surface $=10.75 \mathrm{~nm}$ and $\mathrm{FSR}_{\text {Eth-on surface }}=10.8 \mathrm{~nm}$.

Another issue to take into consideration during the manipulation of the tapered fiber is the position of the anchor points. These must be 

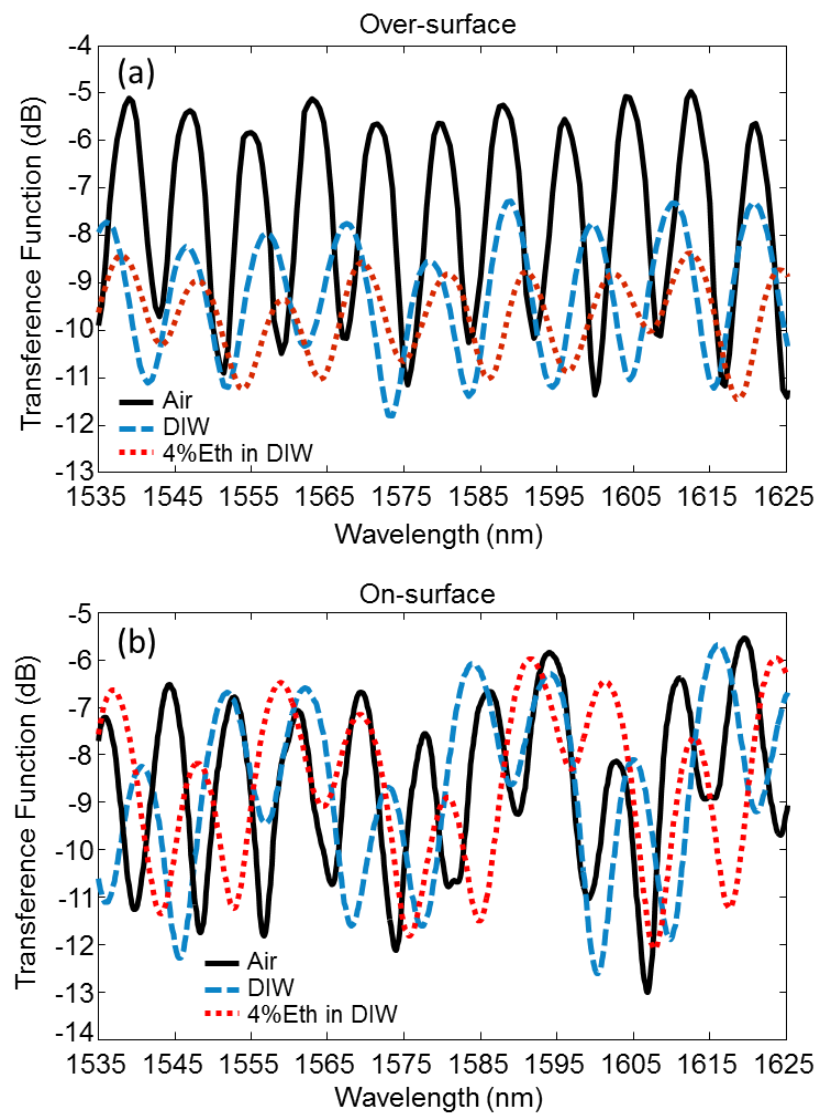

Fig. 3. Transfer function of biconical tapered fibers placed (a) over-surface and (b) onsurface considering air, deionized water (DIW) and 4\% ethanol concentration in DIW as outer mediums.

located in the untapered region. If the anchor points are set in the tapered region, the possibility of breaking the tapered fiber increases dramatically. Moreover, attention must be paid to the creation of bubbles or streams inside the recipient. In the first case, an air bubble in the liquid solution causes an undesired difference in the refractive index of the sensed medium similar to the one produced when the tapered fiber is in contact with a support surface. Also, small currents can be created when introducing liquids in the recipient, which results in a different transfer function because of a modification in the evanescence field. To sum up, in order to assure the correct operation of the refractive index sensor based on tapered fibers it is extremely important to guarantee 
that the tapered fiber will be in exclusive contact with the sensed medium. Otherwise, undesired changes are introduced in the sensing response, leading to considerable measurement errors.

\section{B. Strain sensing}

Handling precautions are required as well when strain sensing measurements based on tapered fibers are carried out. As mentioned above, the transfer function of the tapered fiber depends not only on the index difference between the modes propagating but also on the length of the waist. Thus, stretching of the tapered fiber can be monitored by measuring variations in its transference function. An initial stretching state together with two different elongation points $\mathrm{L}_{1}$ and $\mathrm{L}_{2}$ was analyzed. The most important issue considered here is the presence of curvatures in the taper region when stretching the fiber, as this leads to a completely different sensor response. Two examples of tapered fiber manipulation with and without curvature are depicted in Fig. 4. As can be seen, in the first case the tapered fiber was set completely straight and therefore, when considering different elongation points, the stretching is linearly shared. Conversely, in curved tapered fibers as the one depicted in Fig. 4(b) the transition regions experiences most of the stress, making it easier to break and introducing additional losses in the taper regions.

The transfer function of a straight placed tapered fiber presents the sinusoidal interference pattern provided typically by this type of fiber, as shown in Fig. 5(a). When the tapered fiber is stretched, the sensor response experiences a phase shift to lower wavelengths, resulting in a sensitivity value of $\mathrm{S}_{\text {STRAIN }}=146 \mathrm{pm} / \mu \mathrm{m}$, which is also competitively with state of art value of $95 \mathrm{pm} / \mu \mathrm{m}$ [8]. However, the sinusoidal shape remains constant, differing only in small and local variations in the visibility. This difference originates from slight changes in the shape of the taper region that lead to variations in the coupling process occurring between the fundamental mode and the modes excited in the waist.

However, when considering a curved tapered fiber the transfer function presents lower visibility and an irregular sinusoidal pattern. This is due to the large amounts of optical power that are radiated in the tapered regions. Stretching the tapered fiber to the two different elongation points considered $\mathrm{L}_{1}$ and $\mathrm{L}_{2}$, it can be seen in the transference function of Fig. 5(b) how the sensor response is completely modified. The visibility of the curved tapered fiber increases for increasing stretching. In addition, the transfer function exhibits a nonsinusoidal shape that suggests the existence of more modes than those considered in the straight case. Other issues to take into account include the introduction of small curvatures in the taper-waist interfaces which 

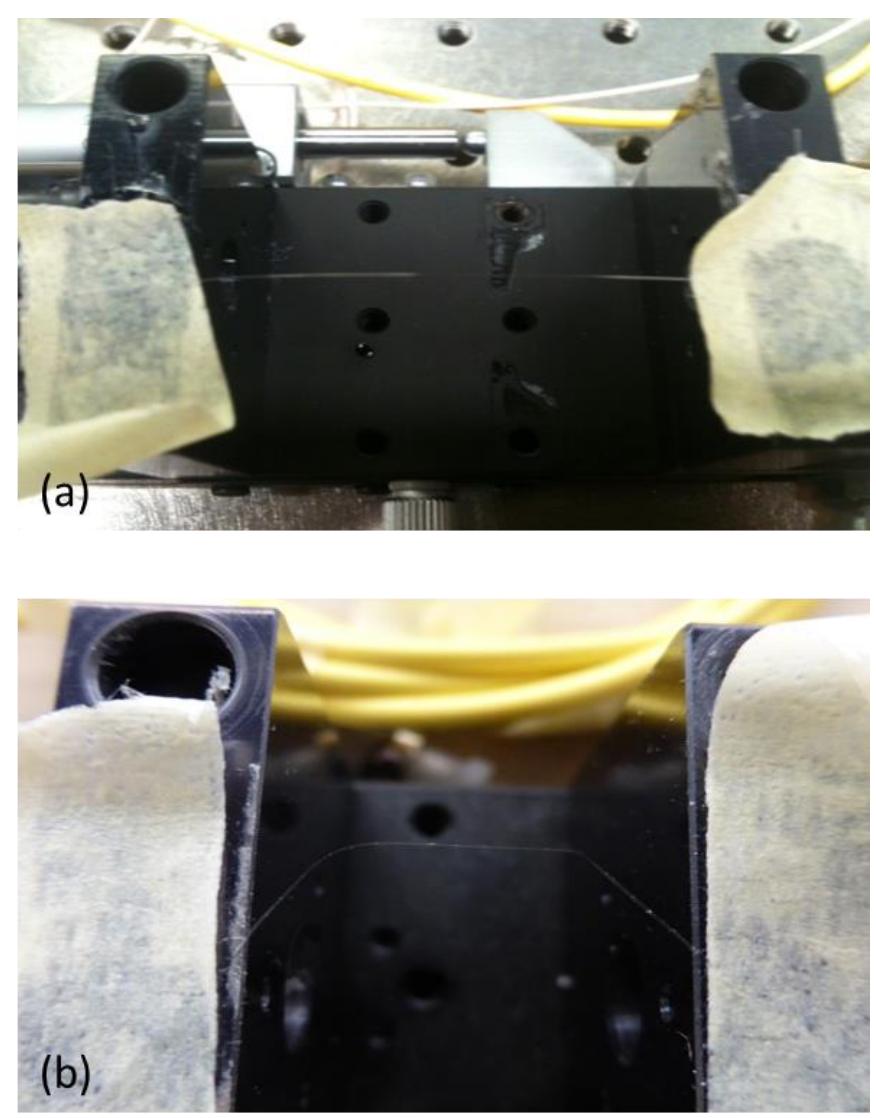

Fig. 4. Examples of (a) straight and (b) curved biconical tapered fibers.

must be avoided as they degrade the sensor's response. Also, the application of an excessive amount of stretching, which will distort or even destroy the tapered fiber.

\section{Conclusion}

In this work, a guideline for sensing measurements that rely on the use of tapered fibers is presented and analyzed. Common manipulation mistakes are discussed and their influence on the sensor performance studied. Refractive index sensing measurements were carried out considering different outer mediums. Two different scenarios, oversurface and on-surface, were taken into account and its influence in the sensor response investigated. Issues as the position of the tapered fiber's anchor points and the creation of bubbles or streams while sensing were 

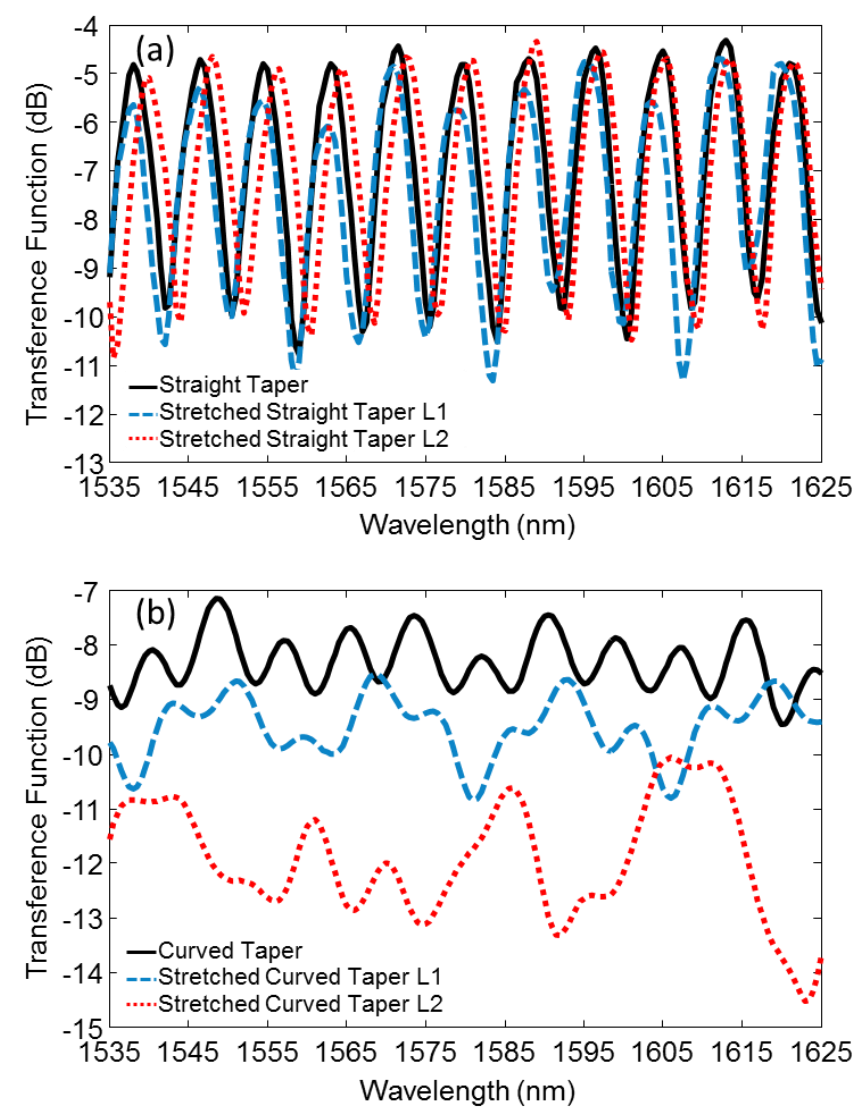

Fig. 5. Transfer function of (a) straight and (b) curved biconical tapered fibers considering three different elongation points.

studied. Moreover, the effect of curvatures in the tapered region of the device during strain sensing measurements is examined. Although the applications discussed here are refractive index and strain sensing other applications which use biconical tapered fibers are expected to benefit from our study.

\section{References}

[1] K. Jedrzejewski, 'Biconical fused taper - a universal fibre devices technology,' Opto-electronics Review 8, 2, 153-159, 2000. 
[2] T. A. Birks and Y. W. Li, 'The shape of fiber tapers,' Journal of Lightware Technology 10, 4, 432-438, 1992.

[3] R. P. Kenny, T. A. Birks, and K. P. Oakley, 'Control of optical fibre taper shape,' Electronic Letters 27, 18, 1654-1656, 1991.

[4] J. Villatoro, D. Monzón-Hernández, and E. Mejía, 'Fabrication and modeling of uniform-waist single-mode tapered optical fiber sensors,' Applied Optics 42, 13, 2278-2283, 2003.

[5] F. Lu and W. H. Knox, 'Generation of a broadband continuum with high spectral coherence in tapered single-mode optical fibers,' Optics Express 12, 2, 347-353, 2004.

[6] B. S. Kawasaki, K. O. Hill, and R. G. Lamont, 'Biconical-taper singlemode fiber coupler,' Optics Letters 6, 7, 327-328, 1981.

[7] S. Mas, J. Palací, P. Pérez-Millán, S. Lechago, D. Monzón-Hernández, and J. Martí, 'All-fiber processing of terahertz-bandwidth signals based on cascaded tapered fibers' Optics Letters 38, 23, 4954-4957, 2013.

[8] K. Q. Kieu and M. Mansuripur, 'Biconical fiber taper sensors,' IEEE Photonic Technology Letters 18, 21, 2239-2241, 2006.

[9] D. Monzón-Hernández and J. Villatoro, 'High-resolution refractive index sensing by means of a multiple-peak surface plasmon resonance optical fiber sensor,' Sensors and Actuators B: Chemical 115, 1, 227-231, 2006.

[10] H.-Y. Lin, C.-H. Huang, G.-L. Cheng, N.-K. Chen, and H.-C. Chui, 'Tapered optical fiber sensor based on localized surface plasmon resonance,' Optics Express 20, 20, 21693-21701, 2012.

[11] T. K. Yadav, M. A. Mustapa, M. H. A. Bakar, and M. A. Mahdi, 'Study of single mode tapered fiber-optic interferometer of different waist diameters and its application as a temperature sensor,' Journal of the European Optical Society 9, 14024-1-14024-5, 2014.

[12] M. I. Zibaii, H. Latifi, Z. Saeedian, and Z. Chenari, 'Nonadiabatic tapered optical fiber sensor for measurement of antimicrobial activity of silver nanoparticles against Escherichia coli,' Journal of Photochemistry and Photobiology B 135, 55-64, 2014.

[13] L. Yao, T. A. Birks, and J. C. Knight, 'Low bend loss in tightly-bent fibers through adiabatic bend transitions,' Optics Express 17, 4, 2692-2967, 2009.

[14] I. M. White, and X. Fan, 'On the performance quantification of resonant refractive index sensors,' Optics Express 16, 2, 10201028, 2008. 
[15] J. García-Rupérez, V. Toccafondo, M.J. Bañuls, J.G. Castelló, A. Griol, S. Peransi-Llopis, and A. Maquieira, 'Label-free antibody detection using band edge fringes in SOI planar photonic crystal waveguides in the slow-light regime,' Optics Express 18, 23, 24276-24286, 2010.

[16] J.-F. Ding, A. P. Zhang, L.-Y. Shao, J.-H. Yan, and S. He, 'Fiber-taper seeded long-period grating pair as a highly sensitive refractiveindex sensor,' IEEE Photonic Technology Letters 17, 6, 1247-1249, 2005.

[17] T. Allsop, F. Floreani, K.P. Jedrzejewski, P.V.S. Marques, R. Romero, D.J. Webb, and I. Bennion, 'Spectral characteristics of tapered LPG device as a sensing element for refractive index and temperature,' Journal of Lightwave Technology 24, 2, 870-878, 2006. 


\title{
Paper G
}

\section{“Curvature Investigation in Tapered Fibers and its Application to Sensing and Mode Conversion"}

\author{
S. Mas, J. Martí, and J. Palací
}

Accepted in Optics and Lasers in Engineering 


\title{
Curvature investigation in tapered fibers and its application to sensing and mode conversion
}

\author{
S. Mas, Javier Martí, and J. Palací \\ Valencia Nanophotonics Technology Center, Universidad Politécnica de \\ Valencia
}

\begin{abstract}
Non-adiabatic tapered fibers are basic photonic components used in a wide range of applications. Here we investigate a way to increase their utility through the controllable bending of one of their tapered sections. The experiments carried out explain, for the first time, the mechanics of this approach showing how these tapers can be used to build more sensitive sensors. Their use as highly efficient mode converters is also discussed.
\end{abstract}

\section{Introduction}

Tapered fibers are versatile devices capable of carrying out a variety of functions from filtering and light coupling to sensing, among others [1]. They consist of a waist located between two tapered sections which are fabricated through heating and pulling [2], [3]. The input mode experiences different effects that depend on the length and shape of the first tapered section. For sections longer than a threshold, which is determined by the characteristics of the fiber, the fundamental mode propagates as usual. These tapers are called adiabatic and its main application is light coupling because of the increased evanescence of the transmitted field [4], [5]. However, when the transition between the fiber and waist section is not smooth the fundamental mode couples to higher-order modes that propagate through the waist [6]. Due to the different propagation velocities, an interference pattern is obtained at the output of the device. These are called non-adiabatic tapers and have been used not only in sensing applications [7]-[9] but also as filters [10][12] and dynamic pulse shapers [13]. 
Extensive work has been done to observe how different parameters such as the tapered fiber's cross-section profile and waist's radius affect the total response of the device. For example, polarization-maintaining [14] and micro-structured fibers [15], [16] have been used to achieve polarization stability thanks to the elliptical core and efficient supercontinuum generation due to the increased light confinement, respectively. However, little work has been done to understand how the tapering sections influence the total response. $\mathrm{Li}$ et al. reported the detection of curvatures in tapered fibers [17], Sun et al. introduced curvatures on the whole structure to implement an amplitude sensor [18] while Luo et al. presented a micro displacement sensor based on a bent microfiber [19]. Because of the bending of the whole structure the results previously reported are not conclusive with respect to the effect of curving the tapering sections since all the structure was curved as a whole. We recently reported some guidelines on the manipulation of tapered fibers for their use as refractive index and strain sensors, where the influence of curvatures on the sensor was studied as a whole [20].

In this paper we present the first results that show the effect of small curvatures in one of the tapering regions on the response of the device. The results obtained show that simple geometrical modifications such as mechanical stretching introduce a highly efficient mode conversion that can be used to reconfigure the properties of the device in interesting ways. This can be used to greatly increase its sensing capabilities and to perform lossless mode conversion in only $1 \mathrm{~mm}$ of propagation.

\section{Principle of Operation}

Figure 1 shows the most relevant modes present in a non-adiabatic tapered fiber with waist length $L$, waist diameter $\rho$ and length of the transition regions Lt. For the geometrical parameters considered here, the fundamental mode splits in two modes, the fundamental cladding mode and a higher order cladding mode. Their different effective indexes originate a spectral sinusoidal fringe pattern at the output of the device due to their different time of arrival. The resulting phase difference between the modes can be expressed as [21]:

$$
\Delta \phi=\frac{2 \pi \Delta n_{e f f} L}{\lambda}
$$

where $\Delta \mathrm{n}_{\text {eff }}$ is the effective refractive indexes difference between the two propagating modes, $\mathrm{L}$ is the interference length and $\lambda$ is the operating wavelength. In this case only two modes are generated, although it is 


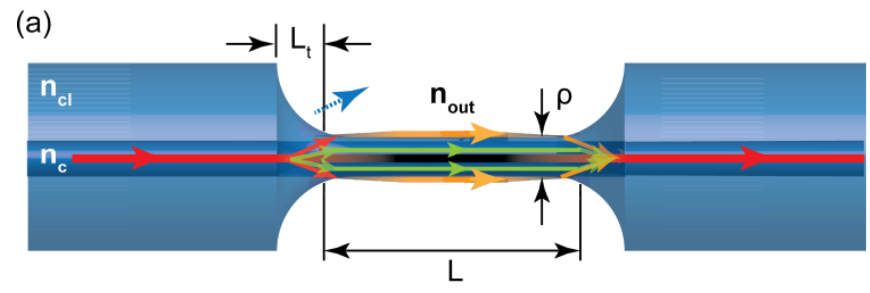

(b)

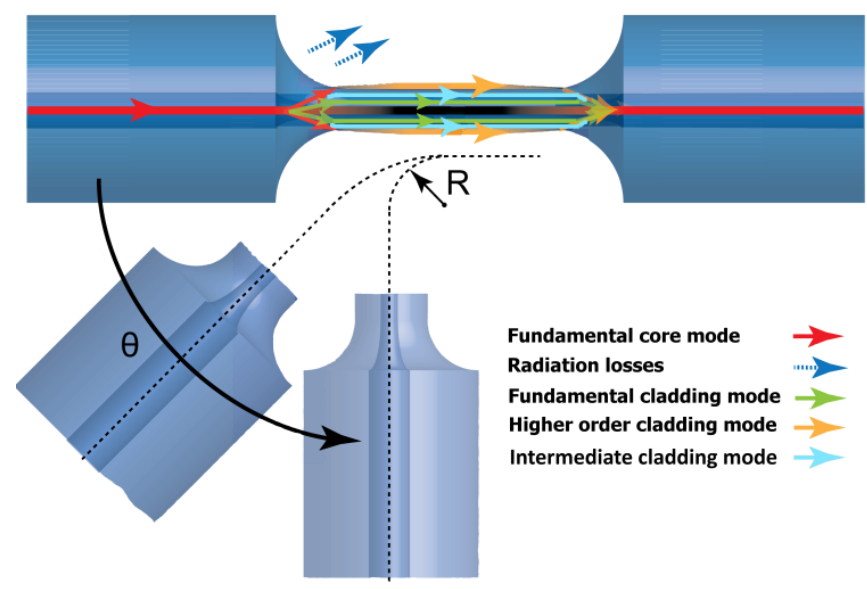

Fig. 1. (a) Straight and (b) curved tapered fiber. $\mathrm{n}_{\mathrm{c}}, \mathrm{n}_{\mathrm{c}}$ and $\mathrm{n}_{\text {out }}$ stand for the refractive indexes of cladding, core and outer medium, respectively.

clear that the transition regions govern how light splits and combines. One simple geometrical modification consists of introducing a curvature of radius $\mathrm{R}$ and $\operatorname{arc} \theta$ into one of the transition regions. In this case, the refractive index of the tapered fiber depends on the bending radius following the expression:

$$
n=n_{0}\left[1+(1+\chi) \frac{x}{R}\right]
$$

where $\mathrm{n}_{0}$ is the refractive index in the corresponding straight tapered fiber, $\chi$ refers to the elasto-optic effect and $\mathrm{x}$ is the transverse coordinate of the curvature [22]. For radii comparable to the size of the modes, the propagating energy will couple outwards, meaning that the higher order cladding mode will experience increased losses while the fundamental cladding mode will couple to additional cladding modes. This is a simple and efficient way of controlling the number of modes present in the waist and therefore the spectral response of the device. For sensing 
applications, it can be used to control the amount of light that interacts with the outer medium and to build cheaper sensors that rely on loss measurement instead of monitoring wavelength shifts of the fringe pattern, as in traditional approaches [23].

\section{Experimental Results and Discussion}

The experimental setup used to characterize tapered fibers is shown in Fig. 2. A tunable laser, model Santec TSL-210F with a tuning range from $1260 \mathrm{~nm}$ to $1630 \mathrm{~nm}$ and emitting $0 \mathrm{dBm}$ of optical power in the telecom band and a power meter model Ando AQ2140 were employed to extract the optical response of the device. Two tapered fibers with parameters $\mathrm{L}=13 \mathrm{~mm}, \rho=18 \mu \mathrm{m}$ and $\mathrm{L}_{\mathrm{t}}=1 \mathrm{~mm}$ were fabricated and fixed on translation stages using glue drops before being put inside of a container which was filled with different fluids.

Results for the straight tapered fiber are shown in Fig. 3. A section of $40 \mathrm{~mm}$ of fiber that includes the tapered fiber was stretched using the translation stage. Taking into account $\mathrm{L}$ and the section-dependent mechanical elasticity of the fiber, approximately half of the stretching applied to the fiber section is applied to the waist. Experimental results were normalized to only consider the stretching experienced by the waist, $\Delta \mathrm{L}$, as a function of the stress applied to the fiber section. Figure $3(\mathrm{a})$ shows the frequency shift $\Delta \omega$ experienced when the tapered fiber is mechanically stretched from its initial state to elongations of $60 \mu \mathrm{m}$ and $100 \mu \mathrm{m}$.

Three different mediums were considered for the refractive index (RI) sensing measurements shown in Fig. 3 (b): air, deionized water (DIW) and a dilution made of DIW and 5\% of ethanol. The refractive

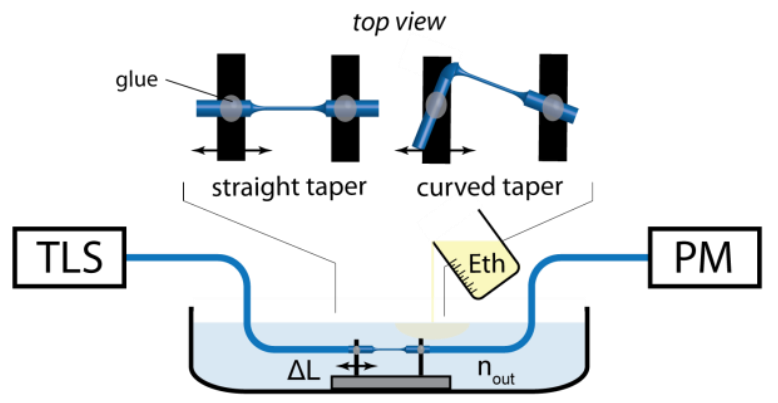

Fig. 2. Experimental setup used to obtain the response of the tapered fibers as a function of strain and outer refractive index. TLS: tunable laser source, PM: power meter. 

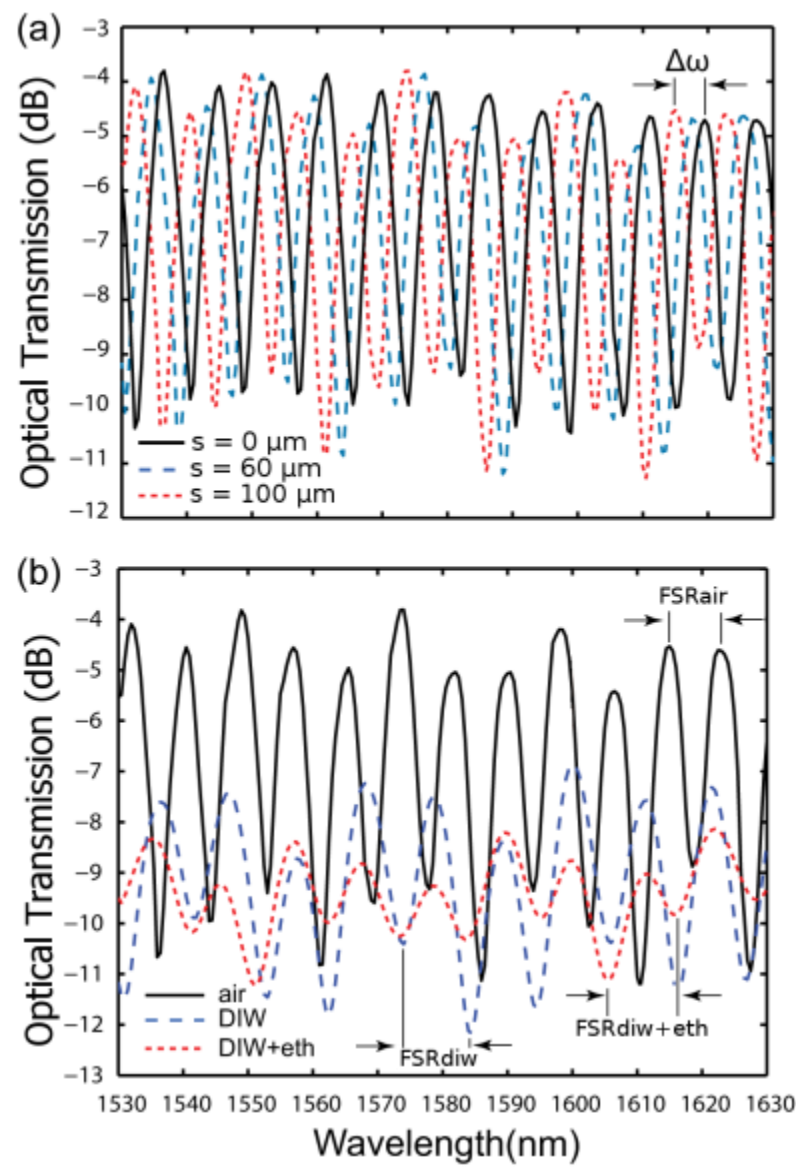

Fig. 3. Spectral response of the straight tapered fiber as a function of (a) strain and (b) surrounding refractive index.

indexes of these mediums at $1550 \mathrm{~nm}$ and ambient temperature are 1, 1.317 and 1.320 [24], respectively. The free-spectral range (FSR) or spectral period of the fringed spectrum increases from $8.35 \mathrm{~nm}$ (air) to $10.98 \mathrm{~nm}$ (DIW) and $11.01 \mathrm{~nm}$ (DIW and ethanol). These measurements provide calculated RIs of 1.315 and 1.320 for DIW and diluted DIW, respectively, by assuming that the higher order cladding mode fully interacts with the outer medium while the fundamental cladding mode interacts very little. According to these experiments a sensitivity of 0.086 $\mathrm{dB} / \mu \mathrm{m}$ is obtained for the strain sensor when using a continuous wave 
(CW) laser emitting in the maximum slope point of the fringe pattern and a power meter to build the sensor, which is competitive with the state of art values [25,26]. Maximum sensitivities of $1000 \mathrm{~dB} / \mathrm{RIU}$ are achieved when using this setup as RI sensor, which are competitive when compared to other refractive index sensing techniques as those shown in [27-29] which reported sensitivity values of $160 \mathrm{~dB} /$ RIU, $304.89 \mathrm{~dB} / \mathrm{RIU}$ and $800 \mathrm{~dB} / \mathrm{RIU}$, respectively.

Results obtained for the curved tapered fiber are shown in Fig. 4. The extremes of the taper are fixed at an angle of $\theta \approx \pi / 2$ and $R$ is varied from a couple of $\mathrm{mm}$ to a few tens of $\mu \mathrm{m}$ as a function of strain. The tapered fiber is fixed so that $\theta$ remains practically constant and independent from R. Figure 4 (a) shows the results for the curved taper surrounded by air under different stretching conditions. For little strain the response is identical to that of a straight tapered fiber because of the large curvature radius. However, losses grow for increased strain and the non-sinusoidal shape of the spectral response observed at higher strain values indicates the presence of more than two modes along the waist.

Further stretching brings the sensor back into 2-mode operation with considerably increased visibility and FSR. Figure 4 (b-c) illustrates the sensor response for different RI values, showing that visibility depends more on strain than on the outer RI. While the shape is not perfectly sinusoidal, which may difficult the measurement of $\Delta \omega$ and $\triangle F S R$, the increase in the visibility can be used to fabricate improved sensors. For example, an increase in the visibility of $10 \mathrm{~dB}$ (as can be obtained for $\mathrm{s}=2400 \mu \mathrm{m}$ when compared to $\mathrm{s}=0 \mu \mathrm{m}$ considering air as the surrounding medium, Fig. 4(a)) provides a tenfold enhancement in the sensor sensitivity.

The transmission response of the tapered fibers is captured at stretching steps of a few microns to observe the evolution of the modes in both straight and curved cases when immersed in different media. When the inverse Fourier transform is applied to the spectral transmission response a good approximation to the impulse response of the device is obtained and the modes present in the waist are seen as time-delayed amplitude peaks because of their different propagation velocities. This is shown in Fig. 5 for both the straight and curved versions of the tapered fiber. Figure 5 (a) illustrates how in the straight taper the same two modes are present, at $\mathrm{t}=0$ ps and $\mathrm{t}=0.8 \mathrm{ps}$, independently of the stretching. However, for stretching values around $150 \mu \mathrm{m}$ some of the power transfers into an intermediate mode at $\mathrm{t}=0.2$ ps. This is probably due to small curvatures in the tapering regions of the sensor which disappear after being tensed. On the other side, Fig. 5 (b) 

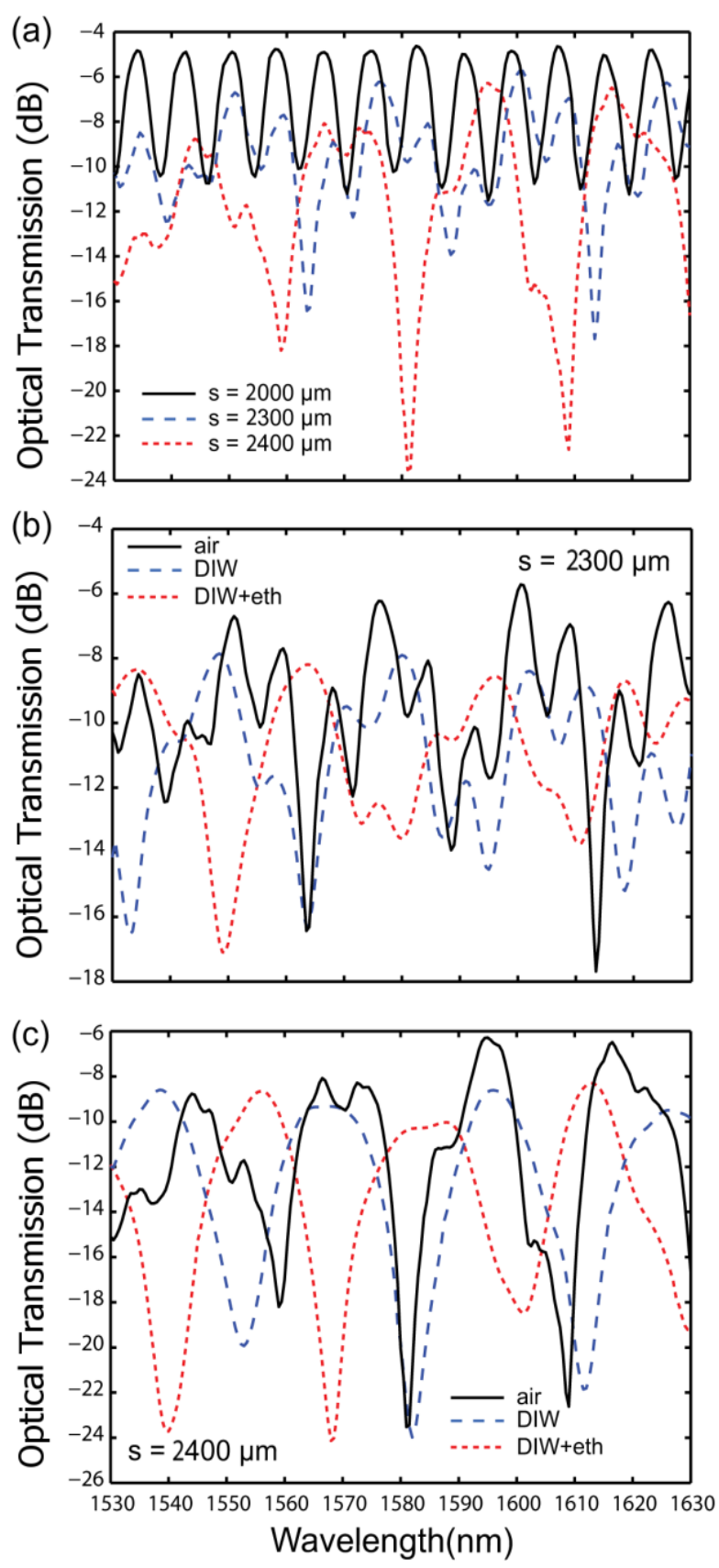

Fig. 4. Spectral response of the curved tapered fiber as a function of (a) strain and (b-c) surrounding refractive index under different strain conditions. 


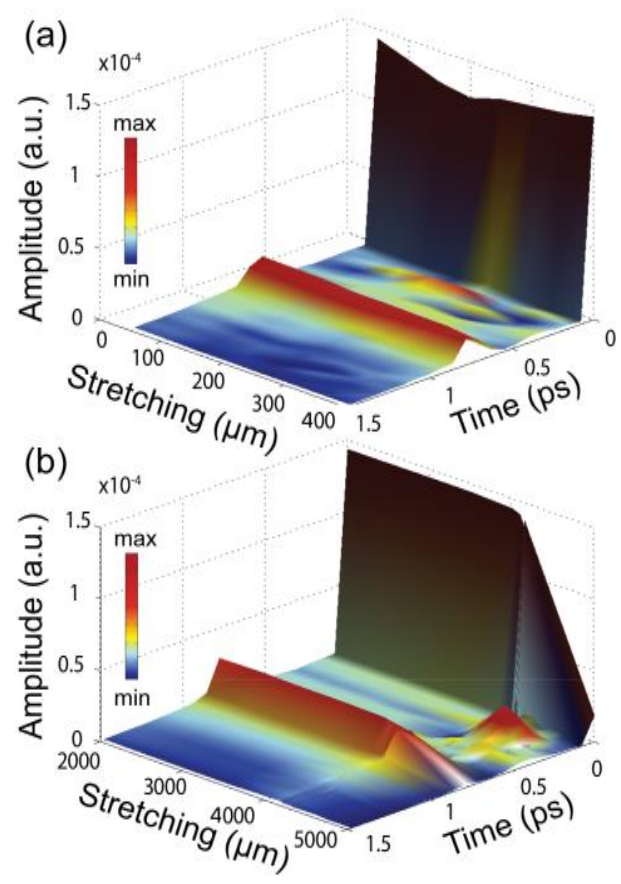

Fig. 5. Inverse Fourier transform of the module of the transference function for (a) straight and (b) curved tapered fibers under different stretching conditions.

shows how the introduction of a curvature in the tapering section has no effect for large radii but introduces mode coupling for small ones. In this case light couples to outer modes; the fundamental cladding mode couples to a higher-order mode while the higher-order cladding mode is radiated. Figure 6 shows the amplitudes of the different modes present in the waist when the different tapers are surrounded by air, DIW and DIW-diluted ethanol. Mode conversion efficiency is high, approaching $100 \%$ when the tapered fiber is surrounded by the dilution of DIW and ethanol. We can distinguish three different operation regions for this device: for large $\mathrm{R}$ values it behaves as a straight tapered fiber and can be used in a traditional sensor arrangement (CW laser). For smaller curvatures (stretching values between $2100 \mu \mathrm{m}$ and $2600 \mu \mathrm{m}$ ) it becomes an efficient mode converter, while for further stretching the total losses increase and it can be used to build cheaper sensor based on incoherent and wideband sources as LEDs. Such a sensor would have a sensitivity of $0.0057 \mathrm{~dB} / \mu \mathrm{m}$. 

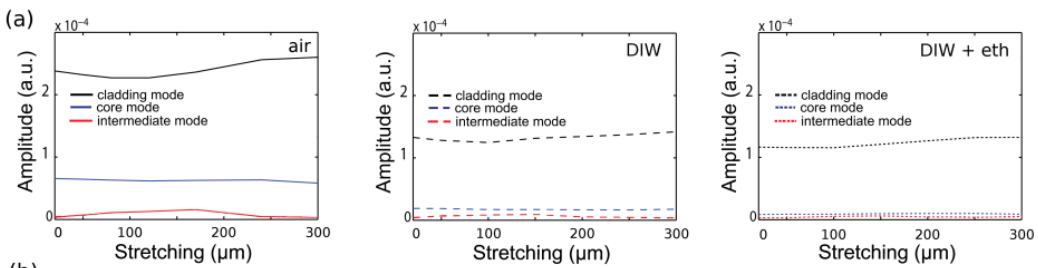

(b)
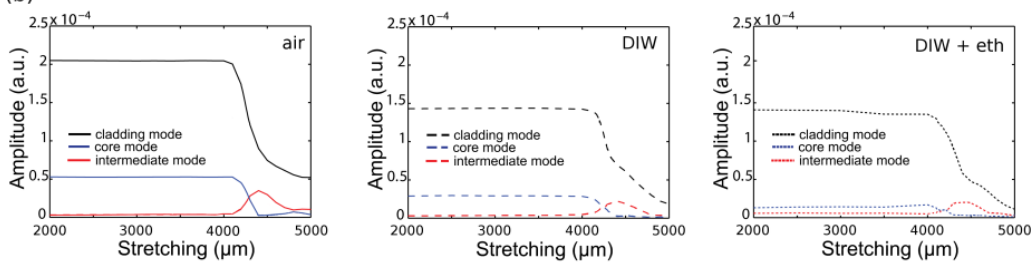

Fig. 6. Mode decomposition as a function of strain in (a) straight and (b) curved tapered fibers surrounded by different mediums: air, DIW and DIW+eth.

\section{Conclusion}

To sum up, we propose a new arrangement in which non-adiabatic tapered fibers can be used to build highly sensitive stress and refractive index sensors. Through the bending of only one of the tapered sections we are able to implement not only more efficient stress sensors but also highly efficient fiber-based mode converters that are only a few millimeters long. The experimental results reported also demonstrate, for the first time, how the mode coupling that takes place in the tapered sections is the main responsible for changes in the transference function of the device while neglecting the influence of light propagation in the waist. Although sensing and mode conversion have been proposed as the main applications of the device, alternative ones as dynamically tunable filtering and pulse shaping may also benefit from this new approach.

\section{References}

[1] J.P. Goure and I. Verrier, 'Optical Fibre Devices', CRC Press, 2010.

[2] J. Villatoro, D. Monzón-Hernández, and E. Mejía, 'Fabrication and modeling of uniform-waist singlemode tapered optical fiber sensors,' Applied Optics 42, 13, 2278-2283, 2003. 
[3] T. E. Dimmick, G. Kakarantzas, T. A. Birks, and P. St. J. Russell, 'Carbon dioxide laser fabrication of fused-fiber couplers and tapers,' Applied Optics 38, 33, 6845-6848, 1999.

[4] D. K. Armani, T. J. Kippenberg, S. M. Spillane, and K. J. Vahala, 'Ultrahigh-Q toroid microcavity on a chip,' Nature 421, 925-928, 2003.

[5] P. Del'Haye, A. Schliesser, O. Arcizet, T. Wilken, R. Holzwarth, and T. J. Kippenberg, 'Optical frequency comb generation from a monolithic microresonator,' Nature 450, 1214-1217, 2007.

[6] J. V. P. Minkovich, and D. Monzon-Hernandez, 'Compact modal interferometer built with tapered microstructure optical fiber,' IEEE Photonic Technology Letters 18, 11, 1258-1260, 2006.

[7] D. Ahuja, and D. Parande, 'Optical sensors and their applications,' International Journal of Scientific Research and Reviews 1, 5, 60-68, 2012.

[8] D. Monzón-Hernández, and J. Villatoro, 'High-resolution refractive index sensing by means of a multiple-peak surface plasmon resonance optical fiber sensor,' Sensors and Actuators B 115, 1, 227-231, 2006.

[9] J. Villatoro, D. Monzón-Hernández, D. Luna-Moreno, 'In-line optical fiber sensors based on cladded multimode tapered fibers,' Applied Optics 43, 32, 5933-5938, 2004.

[10] K. Kieu and M. Mansuripur, 'Tuning of fiber lasers by use of a singlemode biconic fiber taper,' Optics Letters 31, 16, 2435-2437, 2006.

[11] S. Lacroix, F. Gonthier, and J. Bures, 'All-fiber wavelength filter from successive biconical tapers,' Optics Letters 11, 10, 671-673, 1986.

[12] Z. Z. Feng, Y. H. Hsieh, and N. K. Chen, 'Successive asymmetric abrupt tapers for tunable narrowband fiber comb filters,' IEEE Photonic Technology Letters 23, 7, 438-440, 2011.

[13] S. Mas, J. Palací, P. Pérez-Millán, S. Lechago, D. Monzón-Hernández, and J. Martí, 'All-fiber processing of terahertz-bandwidth signals based on cascaded tapered fibers,' Optics Letters 38, 23, 4954-4957, 2013.

[14] Y. Jung, G. Brambilla, and D. J. Richardson, 'Polarization-maintaining optical microfiber,' Optics Letters 35, 12, 2034-2036, 2010.

[15] A. Kudlinski, A. K. George, J. C. Knight, J. C. Travers, A. B. Rulkov, S. V. Popov, and J. R. Taylor, 'Zero-dispersion wavelength decreasing photonic crystal fibers for ultraviolet-extended supercontinuum generation,' Optics Express 14, 12, 5715-5722, 2006. 
[16] N. Vukovic and N. G. R. Broderick, 'Method for improving the spectral flatness of the supercontinuum at $1.55 \mu \mathrm{m}$ in tapered microstructured optical fibers,' Physical Review A 82, 043840, 2010.

[17] W. Li, Z. Hu, X. Li, W. Fang, X. Guo, L. Tong, and J. Lou, 'Highsensitivity microfiber strain and force sensors', Optics Communications 314, 28-30, 2014.

[18] L.-P. Sun, J. Li, Y. Tan, S. Gao, L. Jin, and B.-O. Guan, 'Bending effect on modal interference in a fiber taper and sensitivity enhancement for refractive index measurement', Optics Express 21, 22, 2671426720, 2013.

[19] H. Luo, X. Li, W. Zou, X. Li, Z. Hong, and J. Chen, 'Temperatureinsensitive microdisplacement sensor based on locally bent microfiber taper modal interferometer', IEEE Photonics Journal 4, 3, 772-778, 2012.

[20] S. Mas, J. Martí, and J. Palací, 'Biconical tapered fibers manipulation for refractive index and strain sensing applications', IEEE Sensors Journal 15, 3, 1331-1335, 2015.

[21] M.I. Zibaii, H. Latifi, Z. Saeedian, and Z. Chenari, 'Nonadiabatic tapered optical fiber sensor for measurement of antimicrobial activity of silver nanoparticles against Escherichia coli', Journal of Photochemistry and Photobiology B 135, 55-64, 2014.

[22] L. Yao, T.A. Birks, and J.C. Knight, 'Low bend loss in tightly-bent fibers through adiabatic bend transitions', Optics Express 17, 4, 2692-2967, 2009.

[23] K.Q. Kieu, and M. Mansuripur, 'Biconical fiber taper sensors', IEEE Photonics Technology Letters 18, 21, 2239-2241, 2006.

[24] J. García-Rupérez, V. Toccafondo, M.J. Bañuls, J. GarcíaCastelló, A. Griol, S. Peransi-Llopis, and A. Maquieira, 'Label-free antibody detection using band edge fringes in SOI planar photonic cristal waveguides in the slow-light regime,' Optics Express 18, 23, 24276 24286, 2010.

[25] A.D. Kersey, T.A. Berkoff, and W.W. Morey, 'Multiplexed fiber Bragg grating strain-sensor system with a fiber Fabry-Perot wavelength filter', Optics Letters 18, 16, 1370-1372, 1993.

[26] X. Bao, D.J. Webb, and D.A. Jackson, 'Combined distributed temperature and strain sensor based on Brillouin loss in an optical fiber', Optics Letters 19, 2, 141-143, 1994. 
[27] Y. Gong, T. Zhao, Y. Rao, Y. Wu, and Y. Guo, 'A ray-transfer-matrix model for hybrid fiber Fabry-Perot sensor based on graded-index multimode fiber', Optics Express 18, 15, 15844-15852, 2010.

[28] J. Yuan, C. Zhao, M. Ye, J. Kang, Z. Zhang, and S. Jin, 'A Fresnel reflection-based optical fiber sensor system for remote refractive index measurement using an OTDR', Photonic Sensors 4, 1,48-52, 2014.

[29] T. Allsop, R. Neal, M. Dvorak, K. Kalli, A. Rozhin, and D.J. Webb, 'Physical characteristics of localized surface plasmons resulting from nano-scale structured multi-layer thin films deposited on Dshaped optical fiber', Optics Express 21, 16,48765-18776, 2013. 


\title{
Paper H
}

\section{"Nonlinear Effects Generation in Non- Adiabatically Tapered Fibers"}

\author{
J. Palací, S. Mas, D. Monzón-Hernández, and J. Martí
}

Submitted to Optical Fiber Technology 


\title{
Nonlinear effects generation in non- adiabatically tapered fibers
}

\author{
J. Palací, S. Mas, D. Monzón-Hernández, and J. Martí \\ Valencia Nanophotonics Technology Center, Universidad Politécnica de \\ Valencia
}

\begin{abstract}
Nonlinear effects are observed in a non-adiabatically tapered optical fibre. The designed structure allows for pulse breaking and spectral broadening in approximately a centimetre of propagation using a commercial telecom laser. These devices are simple to fabricate and suitable to generate and control a variety of nonlinear effects in practical applications because they do not experience short-term degradation as previously reported approaches. Experimental and theoretical results are obtained, showing a good agreement.
\end{abstract}

\section{Introduction}

Optical fibre is not only an excellent transmission medium. Doped fibres provide the gain medium necessary to build lasers while others types compensate for the dispersion of standard fibre or increase light-matter interaction through tight confinement of the optical field [1]. In this direction, tapered fibres emerged as a simple way to modify the properties of optical fibres by reducing its cross-section [2]. They have been used for sensing [3], supercontinuum generation [4] and efficient coupling to waveguides and micro-resonators [5, 6], among others.

Tapered fibres are often classified as adiabatic or non-adiabatic according to the transition length. In the first case, the transition is gradual enough for the fundamental mode not to couple to higher order modes. They are the best choice for applications that require different degrees of confinement such as super-continuum generation [4] or evanescent field coupling [6]. On the other hand, in non-adiabatic tapers the waveguide cross-section changes so abruptly that the energy of the fundamental mode splits into several modes that propagate before interfering at the output of the device. Applications of non-adiabatic 
tapers include tunable optical filtering [7] and different types of sensing [3].

In this work, we demonstrate nonlinear generation using nonadiabatically tapered fibres for the first time to our knowledge. Nonlinear effects are intensified thanks to the tailoring of dispersion and to the increased power density obtained when coupling light from the fundamental core mode of a stretched piece of standard single-mode fiber into higher-order cladding modes. Previous approaches rely on adiabatic tapers with micron or submicron diameters [4]. Although their performance is good tapers with such small diameters are degraded and even destroyed in a few days or hours. Our device can be used in a wide range of practical applications because it does not suffer from fast degradation due to its relatively large diameter. The periodic transference function of the device, which can be tuned through optimization of its geometry, makes it especially useful for applications such as wavelength-division multiplexed networks and optical comb generation where a large number of spectrally spaced components are required. Because the approach relies on the stretching of a piece of standard fiber we believe this is one of the simplest solutions that can be found to generate nonlinear effects in fiber-based systems.

\section{Principle of operation}

A tapered fibre can be depicted as a waist of length $L_{w}$ and diameter $\rho$ between two coupling regions of length $\mathrm{T}_{\mathrm{t}}$, as shown in Fig. 1 . When the fibre-to-waist transition is abrupt, the energy carried by the fundamental mode splits into different modes. For the structures considered in this paper two modes, the fundamental cladding mode and a higher order cladding mode, gather most of the optical power. These modes couple back into the fundamental mode of the standard fibre along the second transition. Time-delayed replicas of the input optical field will be obtained at the output of the tapered fibre because of the different propagating modes indexes.

Consider now a dispersion pre-compensated optical pulse propagating through fibre. It narrows during propagation and for powers exceeding a certain value, nonlinear effects such as self-phase modulation become noticeable [8]. The nonlinear power threshold can be drastically decreased through tapering of the standard fibre at the maximum optical intensity point as long as the reduction in the modes' effective area compensates for the losses introduced by power splitting [8]. This mechanism allows for power-efficient generation of nonlinear effects using standard optical fibre. As demonstrated here, the fundamental cladding mode experiences nonlinear propagation for 


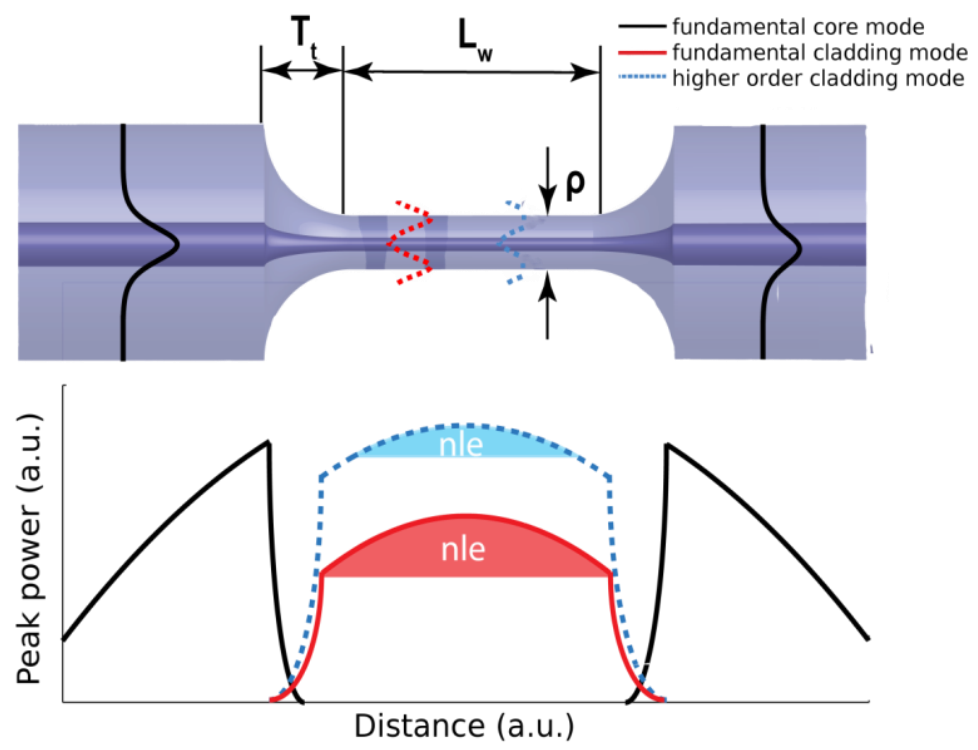

Fig. 1. Power evolution of the main modes existing in the taper for a propagating optical pulse. Shaded area depicted with nle show peak power values exceeding the nonlinear power threshold for each cladding mode. The modes sketched represent a 2-dimensional section of their corresponding intensity profile and appear at different positions along the waist to illustrate their different effective propagation indexes.

lower peak power values because its high spatial confinement results in reduced nonlinear power thresholds.

\section{Experimental results}

The experimental setup is shown in Fig. 2. It consists of a femtosecond fibre laser (FemtoFErb from Toptica Photonics) which emits 100 -fs optical pulses at a repetition rate of $100 \mathrm{MHz}$ with an average power of $100 \mathrm{~mW}$. A piece of dispersion compensating fibre (from OFS) is used to pre-compensate for the second-order dispersion of 10 meters of standard fibre (SMF-28e+ from Corning). Dispersion precompensation was carried out so non-linear effects could not be observed before the tapering of the fibre. At the end of the link, the optical fibre was tapered through heating and controlled pulling. For our design, the transitions regions present an exponential profile with 


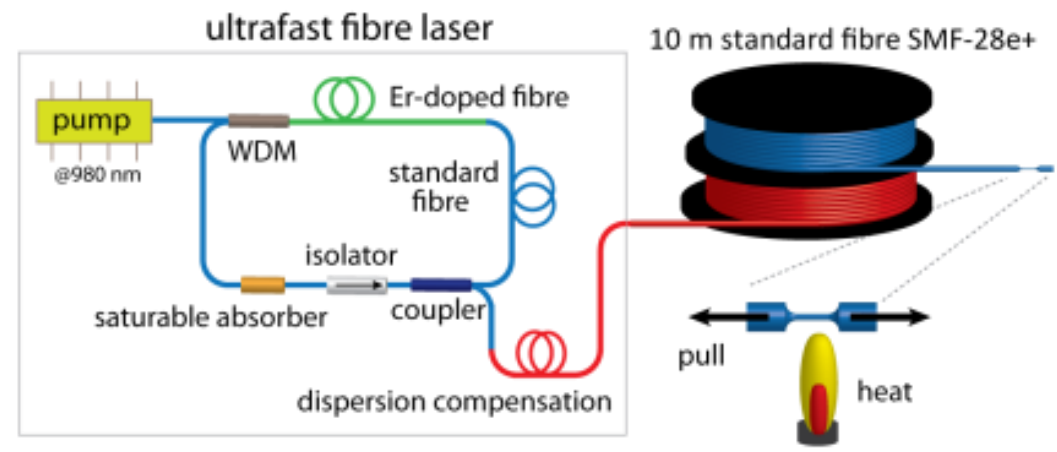

Fig. 2. Experimental setup used to nonlinear generation. The end of the fiber is tapered through a heating and pulling process.

$\mathrm{T}_{\mathrm{t}}=1 \mathrm{~mm}$ while the waist length and diameter are $\mathrm{L}_{\mathrm{w}}=13 \mathrm{~mm}$ and $\rho=18$ $\mu \mathrm{m}$, respectively. The taper was large enough to be visible using a standard camera, as shown in Figure 3 where its transference function, measured using a tunable laser and a power meter, is also included. The taper has $3 \mathrm{~dB}$ insertion losses and free spectral range of $6 \mathrm{~nm}$. Its transference function is practically sinusoidal implying that two modes gather most of the energy. In these structures, the spectral fringe period depends mainly on the waist length, making robust tapered fibres with fringe periods between a few $\mathrm{nm}$ and $200 \mathrm{~nm}$ available. This would be hardly attainable using other technologies.

The nonlinear Schrödinger equation was solved using the split-step Fourier method [8] to simulate pulse propagation through the fibre link. A 100 -fs sech ${ }^{2}$ optical pulse was considered for the simulation in order to realize an accurately comparison with the experiments. Simulations include second- and third-order dispersion, losses and different nonlinear effects such as self-phase modulation. Typical parameter values were used for the standard and dispersion compensation fibre. In addition, effective areas and propagation indexes of the different taper cross sections were calculated using commercial photonics simulation software. Values of 1.4475 and 1.444 are considered for the core and cladding materials, respectively. The nonlinear index is $2.6 \cdot 10^{-20} \mathrm{~m}^{2} / \mathrm{W}$. For the standard fiber we consider a group velocity dispersion (GVD) of $17 \mathrm{ps} / \mathrm{nm} \cdot \mathrm{km}$ and a dispersion slope of $0.13 \mathrm{ps}^{3} / \mathrm{km}$. These values become $50 \mathrm{ps} / \mathrm{nm} \cdot \mathrm{km}$ and $0.1 \mathrm{ps}^{3} / \mathrm{km}$ in the waist of the taper. These values are referred to a wavelength of $1550 \mathrm{~nm}$. The effective mode area of the standard fiber's core mode is $80 \mu \mathrm{m}^{2}$, which is reduced 


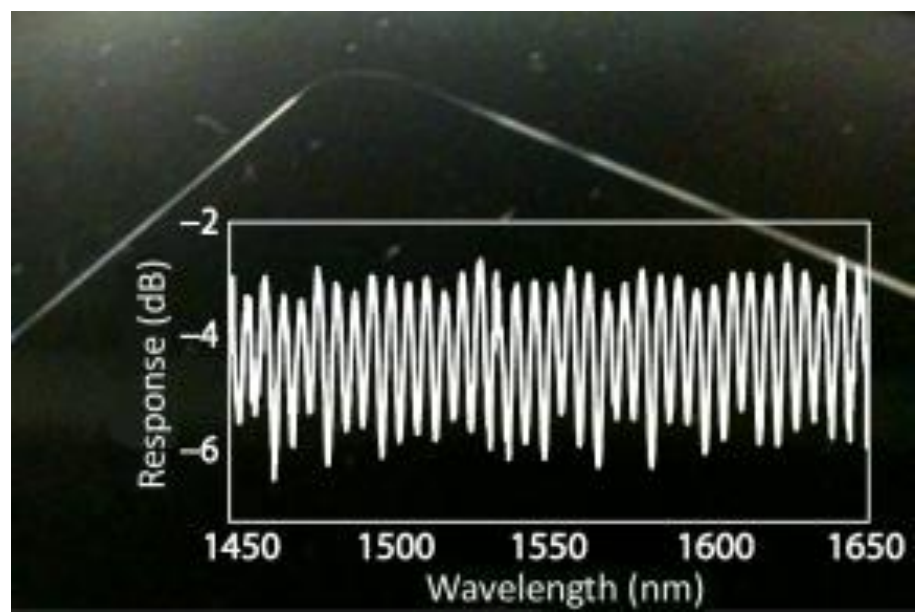

Fig. 3. Photograph of the tapered fibre fabricated and its optical characterization by means of a tunable laser and a power meter.

to half this amount for the cladding modes. The two cladding modes that propagate through the waist have effective refractive propagation indexes of 1.4444 and 1.4214, respectively. Figure 4 shows the theoretical results of the nonlinear Schrödinger equation in both time and spectrum domains. Chirped pulse distribution considers the fibre link without the tapered fibre. The peak power can be increased up to some tens of $\mathrm{kW}$ with no evidence of nonlinear effects thanks to prechirping of the pulses. After including the tapered fibre in the simulator, two pulses with their associated fringed spectrum can be observed. As can be seen, nonlinear generation is not appreciable for input peak power values up to $7 \mathrm{~kW}$. This value agrees well with theory, which for our waist diameter and length estimates that nonlinear effects should become appreciable for peak powers above $7 \mathrm{~kW}$ [8]. After injecting pulses with a peak power of $10 \mathrm{~kW}$ nonlinear effects become noticeable. Typical features of self-phase modulation such as pulse breaking and spectral widening can be clearly appreciated in the time and spectrum domain, Fig. 4(a) and Fig. 4(b), respectively.

Figure 5 shows the experimental results. Chirped pulse distribution allows for linear delivery of the optical pulses. After fibre tapering, the resulting spectrum for peak powers below the nonlinear threshold corresponds with the filtered optical source. By using $10 \mathrm{~kW}$ pulses nonlinear effects, mainly self-phase modulation, reshape the spectrum 
(a)

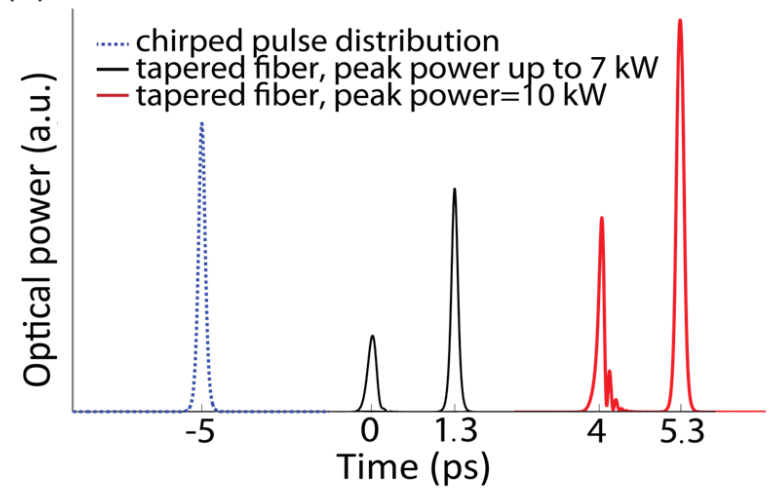

(b)

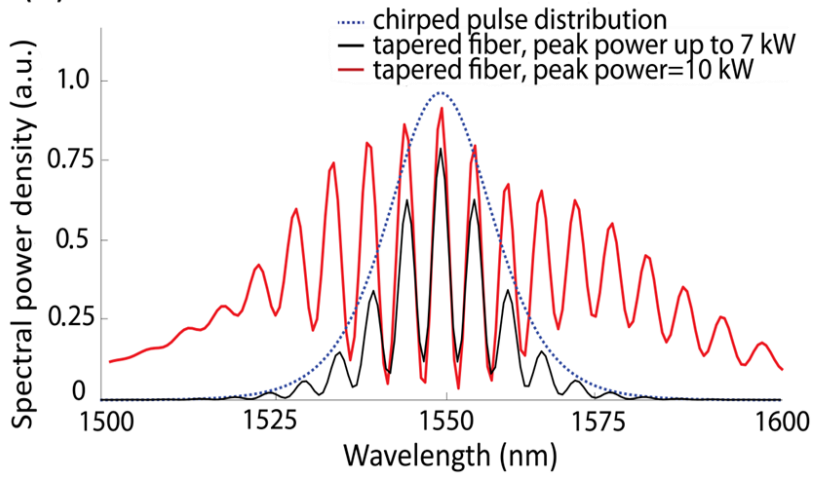

Fig. 4. Numerical solution of the nonlinear Schrödinger equation for a pulse that propagates through the fiber link, (a) Time-domain results and (b) Spectrum-domain results.

and increase the visibility of some fringes. The inset in Fig. 5 shows the autocorrelation intensity traces obtained. As can be observed, nonlinear effects introduce a change in the amplitude ratio between the peaks. Although sub-pulses originating from self-phase modulation cannot be resolved using our intensity autocorrelator the reduction in the relative delay between main and secondary peaks suggests pulse breaking. Differences between the experiments and simulations arise due to differences between the real and estimated parameters and because of the limited resolution of the experiments. However, the main features that indicate nonlinear generation such as asymmetric spectrum expansion and variable fringe visibility are shown in both cases while appearing at the same optical power levels. Because the experiments 


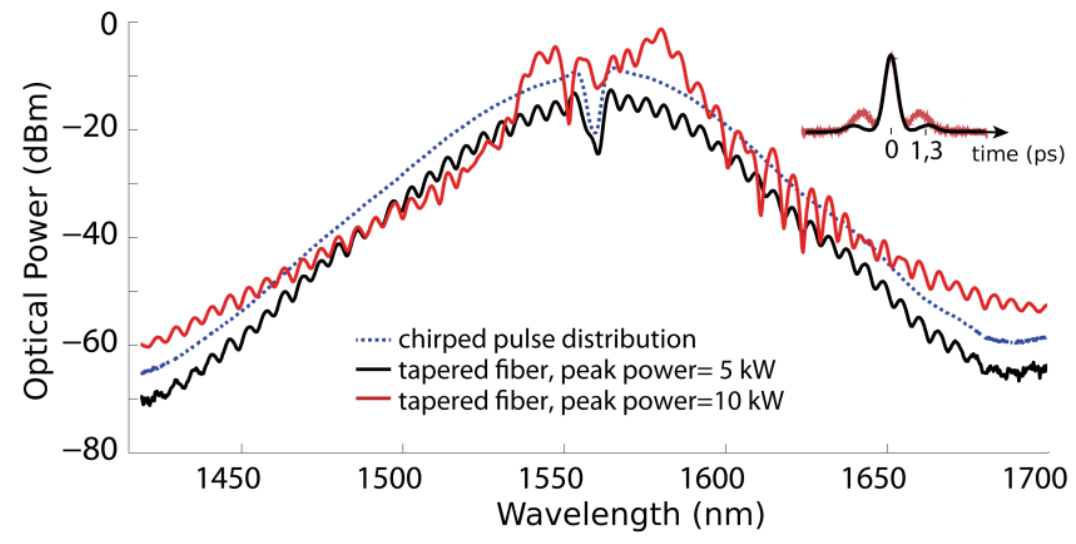

Fig. 5. Spectra showing linear and nonlinear operation of the taper. Corresponding optical intensity autocorrelation traces are shown in the upper right corner.

aimed to find the power threshold for nonlinear generation the expansion of the spectrum is not noticeable for the central components of the spectrum, although it is visible for those around $1450 \mathrm{~nm}$ and $1650 \mathrm{~nm}$. The asymmetry observed in the output response is due to the chirp imposed on the input pulse when the propagating pulse experiences self-phase modulation [9]. The results obtained show that the power required to generate nonlinear effects is reduced by a factor of 8 when compared with the power levels that would be required using standard optical fiber.

\section{Conclusion}

In this work nonlinear generation using non-adiabatically tapered fibres is proposed and demonstrated. The device fabricated considerably reduces the minimum optical power required to generate nonlinear effects as demonstrated through simulations and experiments. It is simple to fabricate, made of standard fibre and robust enough to be useful in practical applications where previous approaches based on adiabatic tapers with micron or submicron radii are not suitable due to their short lifetimes. Because of its interferometric nature and tunable spectral period this device should be useful in several applications, including wavelength generation in wavelength-division multiplexing systems. 


\section{References}

[1] G.P. Agrawal, 'Fiber-optic communication systems', WileyInterscience, 2002.

[2] J. Villatoro, D. Monzón-Hernández, and E. Mejía, 'Fabrication and modeling of uniform-waist single-mode tapered optical fiber sensors', Applied Optics 42, 13, 2278-2273, 2003.

[3] P. Lu, L. Men, K. Sooley and Q. Chen, 'Tapered fiber Mach-Zehnder interferometer for simultaneous measurement of refractive index and temperature', Applied Physics Letters 94, 13, 131110, 2009.

[4] S. Leon-Saval, T. Birks, W. Wadsworth, P.St.J. Russell, and M. Mason, 'Supercontinuum generation in submicron fibre waveguides' Optics Express 12, 13, 2864-2869, 2004.

[5] T. Alder, A. Stöhr, R. Heinzelmann, and D. Jäger, 'High-efficiency fiber-to-chip coupling using low-loss tapered single-mode fiber', IEEE Photonic Technology Letters 12, 8, 1016-1018, 2000.

[6] D.K. Armani, T.J. Kippenberg, S.M. Spillane, and K.J. Vahala, 'Ultrahigh-Q toroid microcavity on a chip', Nature 421, 925-928, 2003.

[7] S. Mas, J. Palací, P. Pérez-Millán, S. Lechago, D. Monzón-Hernández, and J. Martí, 'All-fiber processing of terahertz-bandwidth signals based on cascaded tapered fibers', Optics Letters 38, 23, 4954-4957, 2013.

[8] G.P. Agrawal, 'Nonlinear fiber optics', Academic Press, 2001.

[9] R.H. Stolen, and C. Lin, 'Self-phase-modulation in silica optical fibers', Physical Review A 17, 4, 1448-1453, 1978. 